\title{
HISTORIANS' EXPERIENCES USING DIGITIZED ARCHIVAL PHOTOGRAPHS AS EVIDENCE
}

\author{
Alexandra M. Chassanoff
}

A dissertation submitted to the faculty of the University of North Carolina at Chapel Hill in partial fulfillment of the requirements for the degree of Doctor of Philosophy in the School Information and Library Science.

Chapel Hill

2016

Approved by:

Christopher A. Lee

Sandra Hughes-Hassell

Matthew Kirschenbaum

Ryan Shaw

Helen Tibbo 
(C)2016

Alexandra M. Chassanoff

ALL RIGHTS RESERVED 


\begin{abstract}
Alexandra M. Chassanoff: Historians' Experiences Using Digitized Archival Photographs as Evidence (Under the direction of Christopher A. Lee)

Widespread digitization has presented scholars with unprecedented access to archival sources. In particular, the availability of archival photographs through online collections has been championed as an opportunity to fill in underrepresented histories absent from archival collections. Yet the degree to which scholars are using digital visual sources, and how they are using them, is relatively unexplored in the literature. In part, this can be attributed to the difficulties of modeling visual information use; no empirical models currently link scholarly interpretive practices to how scholars actually use visual materials.
\end{abstract}

This dissertation sought to address these gaps in the literature by examining the experiences of one group - self-identified historians using digitized archival photographs as evidence in their scholarly activities. This study uses an embedded case study approach to explore how and why historians use images in the construction of their arguments. Fifteen participants were recruited during the spring and summer of 2015. I conducted semi-structured interviews with each participant, eliciting descriptions about their image practices and specific experiences related to image use. I used thematic analysis and thematic synthesis to reveal salient aspects of historians' experiences as they interpret and decide to use (or not use) materials. To strengthen and verify the analysis, I used triangulation strategies at different stages in the study.

The results of this exploratory research can be used to inform designs for archival description and access, and to provide guidance for historical image use. In particular, my 
findings disclose the various factors that matter to historians in their experiences interacting with archival photographs in digital environments. Examples of image use are largely absent from the historical literature; the case studies presented in this research help to illustrate the functional ways historians currently use digitized photographs in both research and instructional capacities. Each case also sheds light on the processes and practices historians employ as they construct evidence from photographs and supplementary materials.

This research also makes important theoretical contributions to the LIS literature. While there has been unquestionable growth in access to digital sources, few empirical studies have examined scholarly interactions with digitized archival materials. Toward that end, this study introduces a conceptual framework for exploring how and why historians use digitized photographs. It presents a holistic methodology that focuses attention on information experiences as spaces for meaning-making in digital environments. Attending to my participants' experiences using photographs as historical evidence helped to reveal, in the words of Gregory Bateson, "the difference that makes a difference. 
To my parents Judy and Arnie 


\section{ACKNOWLEDGEMENTS}

I am immensely grateful to the fifteen participants who contributed their time and perspective over the course of fifteen months to participate in this research study. Their particular insights and lived experiences were essential components in telling this story.

This dissertation would not have been possible without the guidance, advice, and patience of my advisor, Dr. Christopher (Cal) Lee. Cal continually kept an open mind as I talked through various ideas and strategies, providing structure and endless suggestions for sculpting a dissertation topic out of vague connections of interest. Simply put, Cal made me a better writer, editor, and researcher. I am grateful for his support and guidance. I am also extraordinarily lucky to have been able to assemble a superior group as my committee members: Matthew Kirschenbaum, Sandra Hughes-Hassell, Ryan Shaw, and Helen Tibbo. Your careful attention and feedback at different points in this process were essential contributions. Thank you to SILS faculty member Diane Kelly for providing methodological guidance early-on. I'd also like to thank my good friend and colleague, Kaitlin Costello, for endless support, timely feedback, and simply "just getting it."

Friends and loved ones, thank you for being there. I could not have done this without you. 


\section{TABLE OF CONTENTS}

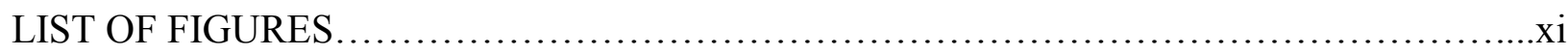

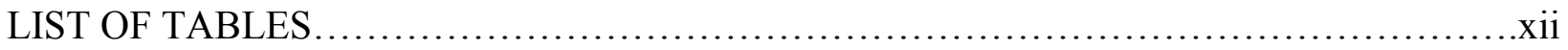

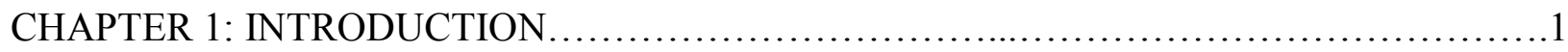

1.1 Statement of the Problem...........................................................

1.2 Purpose of the Study and Research Questions......................................... 5

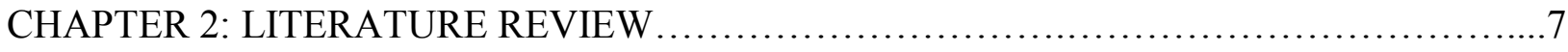

2.1 Information Practices of Historian Scholars Using Primary Source Materials..............7

2.1.1 Research practices of humanities scholars.................................. 8

2.1.2 Information behavior of historians in archival settings.......................10

2.1.3 Scholarly use of digital collections.......................................14

2.2 Describing Photographs in Archival Collections: History, Methods, and Implications...16

2.2.1 Institutions and photographic materials....................................17

2.2.2 Descriptive approaches to photographic print materials.......................18

2.2.3 Descriptive standards for digital image collections...........................28

2.2.4 The problem with visual materials: Implications for archival access.............36

2.3 LIS Approaches to Studying Image Use........................................... 39

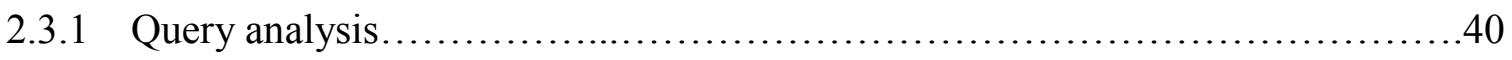

2.3.2 Task-based analysis.......................................................

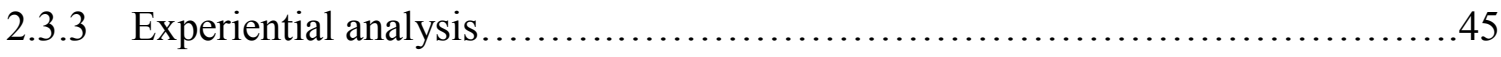


2.4 Historians and the Use of Photographs as Evidence.................................50

2.4.1 Concepts of evidence in modern historiography..............................51

2.4.2 Historians and photographic use ..........................................60

2.4.3 How historians approach photographs as visual evidence......................62

2.4.4. Methods for analyzing historical photographs...............................67

2.4.5 Case studies: Historians using photographs as visual evidence..................68

2.4.6 The current state of historical practice: Summary and implications..............72

2.5 The Experience of Information: Methodological Approaches..........................73

2.5.1 Information as material practice: a short history ............................75

2.5.2 Information use in digital environments..................................... 81

2.5.3 Theoretical approaches to understanding experiences in digital environments....84

2.5.3 Information use as emergent experience.....................................91

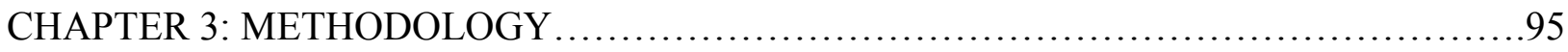

3.1 Methodological Approach.......................................................... 95

3.2 Rationale for Methodological Approach..........................................96

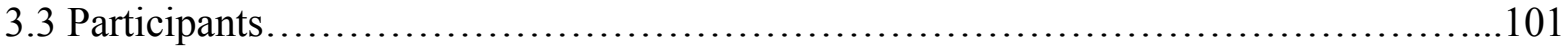

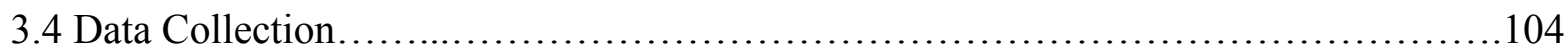

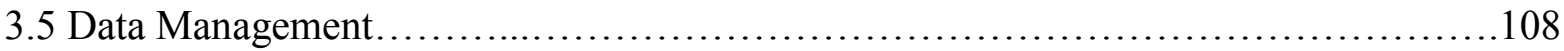

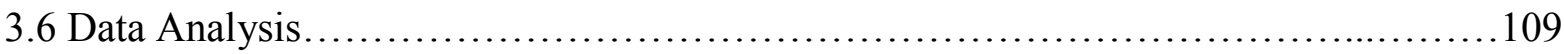

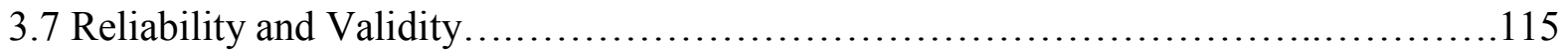

CHAPTER 4: FINDINGS AND DISCUSSION ......................................... 120

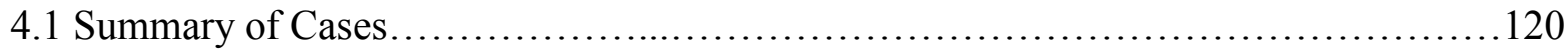

4.2 Descriptive and Analytical Themes of Historians' Experiences.......................132 
4.3 Potential Image Use.............................................................

4.3.1 Research practices in digital environments .................................135

4.3.2 Motivations in potential image use..........................................139

4.3.3 Factors that matter in potential image use..................................142

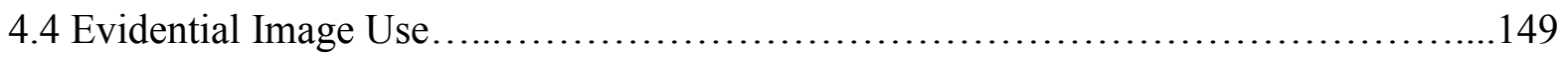

4.4.1 Constructing modes of historical evidence...................................151

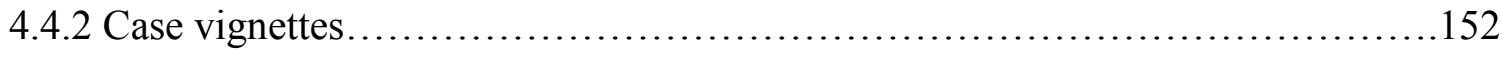

4.5 A Conceptual Framework for Studying Historians' Image Use..........................190

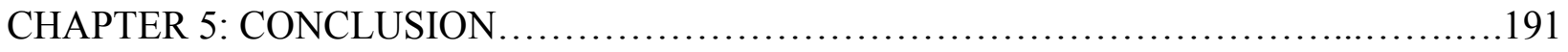

5.1 Significance of the Study .............................................................

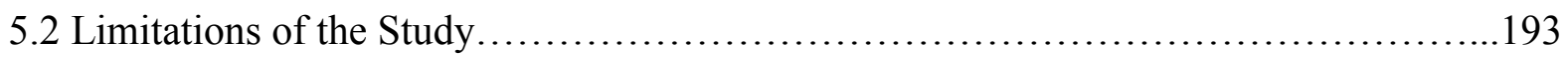

5.3 Implications and Future Work..................................................... 196

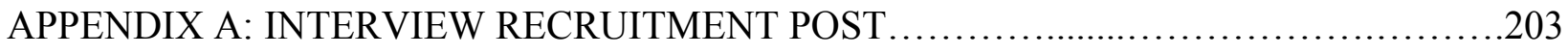

APPENDIX B: INTERVIEW RECRUITMENT EMAIL.................................204

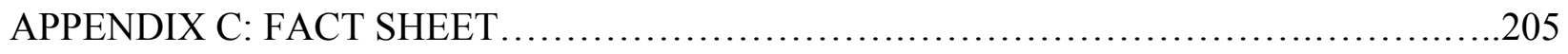

APPENDIX D: VERBAL CONSENT SCRIPT ......................................... 207

APPENDIX E: SEMI-STRUCTURED INTERVIEW PROTOCOL...........................208

APPENDIX F: PHOTO-ELICITATION INTERVIEW PROTOCOL ..........................210

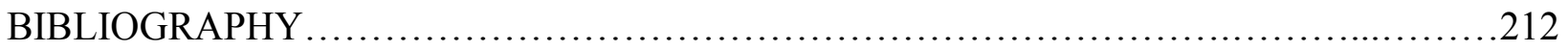




\section{LIST OF FIGURES}

Figure 1. Jorgensen's twelve types of image attributes............................27

Figure 2. Stages of analysis and interpretation..................................... 114

Figure 3. Thematic analysis and synthesis of historians' experiences...................132

Figure 4. Thousand Mile Tree, 1000 Miles West of Omaha...........................154

Figure 5. $\quad$ Thousand Mile Tree, 1000 Miles West of Omaha, Looking West.......................155

Figure 6. $\quad$ Thousand Mile Tree, 1000 Miles West of Omaha, Looking East................156

Figure 7. Debris at P.R.R. Stone Bridge...................................... 157

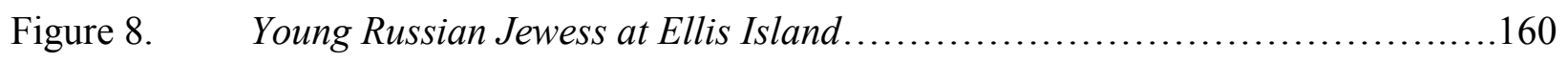

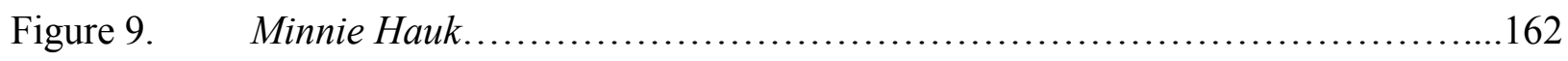

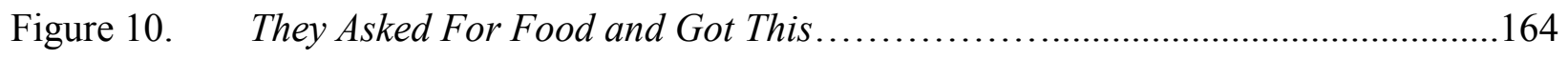

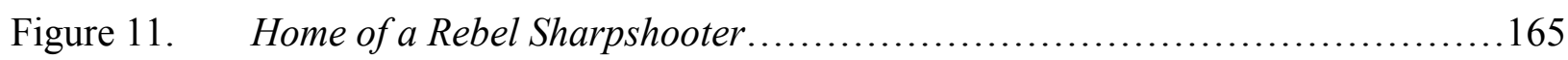

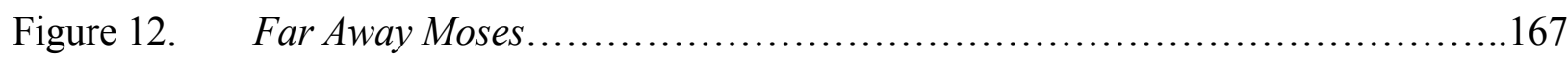

Figure 13. Japanese Captured in Raid........................................... 168

Figure 14. Griffin Children of West Alabama Land Use Project........................169

Figure 15. High School Student Taylor Washington is Arrested........................ 170

Figure 16. Clip from power-point presentation...................................... 172

Figure 17. Sailors dancing with each other aboard the U.S.S. Olympia...................173

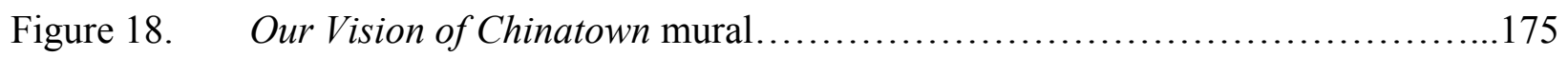

Figure 19. Carl Akeley creating model of Bull Elephant................................176

Figure 20. William Temple Hornaday.......................................... 177

Figure 21. African-American Musicians...................................... 178 
Figure 22. Camp of $31^{\text {st }}$ Pennsylvania Infantry near Washington DC.

Figure 23. Vase, or Dial Rocks, Red Buttes......................................181

Figure $24 \quad$ Front Office of Wright Company...................................... 182

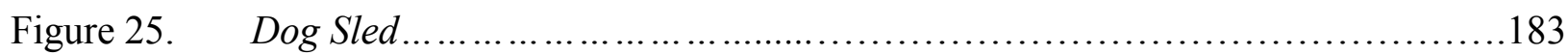

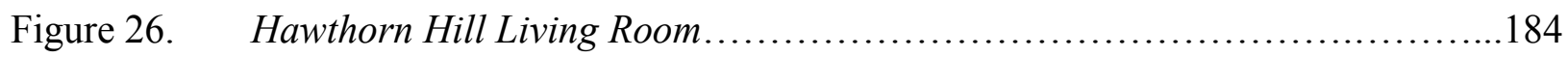

Figure 27. Orville's Reading Chair............................................. 185

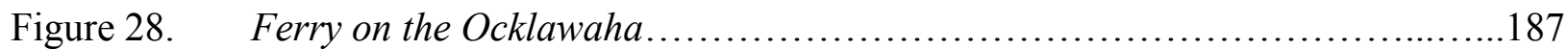

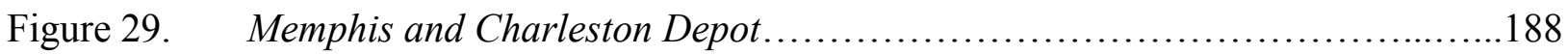

Figure 30. A conceptual framework for studying historians' image use..................190 


\section{LIST OF TABLES}

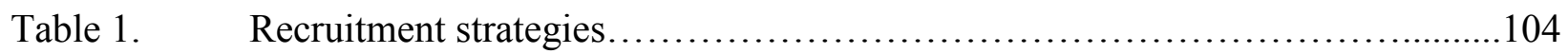

Table 2. Participants by primary research area, sample teaching course, and degree......106

Table 3. Participants by title, department, and institutional affiliation...................120

Table 4. Analytical themes in potential image use................................134

Table 5. Historical research practices in digital environments....................... 135

Table 6. Motivations in potential image use.....................................139

Table 7. Factors that matter in potential image use................................143

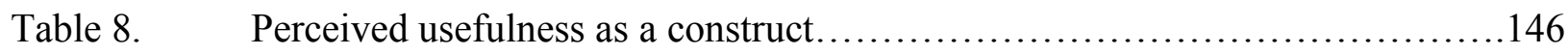

Table 9. Characterizing evidential image use................................ 151 


\section{CHAPTER 1: INTRODUCTION}

There have been widespread changes in access to primary source materials during the last decade. Institutions around the world have active digitization programs, and they regularly provide online access to digital materials. A 2012 survey of the Association of Research Libraries' (ARL) special collections and archives in the US and Canada found that $80 \%$ of institutional directors agreed that digitized special collections are "critical to our current strategic direction(s)."1 In particular, visual materials have grown dramatically in size. Since 1998, special collections have seen a 300\% mean increase in holdings of visual materials and a $76 \%$ increase in use. ${ }^{2}$

Presumably, such changes in the information environment have impacted how scholars interact with, and use, primary source materials. In the discipline of history, where scholars craft arguments from examination of archival sources, increased access to materials through digitization has been celebrated as the "democratization of historical research." 3 Postmodernists" and poststructuralists' critical examination of the historical canon as discourse helped orient historians away from the study of significant figures and toward the "history of ordinary people

\footnotetext{
${ }^{1}$ Nancy Maron and Sarah Pickle, Appraising Our Digital Investment: Sustainability of Digitized Special Collections in ARL Libraries (Washington, DC: Association of Research Libraries, 2013): 11.

${ }^{2}$ Jackie M. Dooley and Katherine Luce, Taking Our Pulse: The OCLC Research Survey of Special Collections and Archives. (Dublin, Ohio: OCLC Research, 2010): 10, 37. In their report, Dooley and Luce define visual materials as architectural materials, drawings, ephemera, paintings, photographs, postcards, prints, and slides/transparencies.

${ }^{3}$ Cheryl Bolick, "Digital Archives: Democratizing the Doing of History," The International Journal of Social Education 21, no. 1(2006): 122.
} 
and everyday life."4 These shifts in focus toward new kinds of subjects and subject matter encouraged the use of different types of source materials as historical evidence. ${ }^{5}$

Historians studying class, gender, and race regard photographic archives as critical resources in establishing the so-called "new history." Photographs in archives can provide evidence to fill in under-developed, underrepresented or misrepresented cultural histories. In his introduction to a special issue in the Journal of Pacific History from 2006 on photography and history, Quanchi explains that "visual histories based on photographic evidence might reveal for the first time, quite different stories, incidents, memories, attitudes and cross-cultural relationships."7 Writing in 1988 in the Radical History Review, Eric Margolis argues, "Photographic evidence has the potential not just to illustrate but to illuminate; historical processes, meanings, ideologies and conflicts can be gleaned from photographic analysis, just as they are generated from the study of written texts." 8

Yet with few exceptions, historical scholarship has failed to seize upon the photograph. Despite the so-called "visual turn," Sarah Farmer explains, "Historians of the western experience in the nineteenth and twentieth centuries still overwhelmingly depend on text-based archives."9

\footnotetext{
${ }^{4}$ Katharine Martinez, "Imaging the Past: Historians, Visual Images and the Contested Definition of History," Visual Resources 11, no. 1 (1995): 24.

${ }^{5}$ Philipp Muller, "Understanding History: Hermeneutics and Source-Criticism in Historical Scholarship," in Reading Primary Sources: The Interpretation of Texts from 19th and $20^{\text {th }}$-Century History, eds. Miriam Dobson and Benjamin Ziemann (Abingdon, UK: Routledge, 2009), 23.

${ }^{6}$ The term "new history" can be traced to the writings of James H. Robinson, a historian writing in the early $20^{\text {th }}$ century. See: James H. Robinson, The New History: Essays Illustrating the Modern Historical Outlook (New York: Macmillan, 1912).

${ }^{7}$ Max Quanchi, "Visual Histories and Photographic Evidence," The Journal of Pacific History 41, no.2 (2006): 165. doi:10.1080/00223340600826052.

${ }^{8}$ Eric Margolis, "Mining Photographs: Unearthing the Meanings of Historical Photos," Radical History Review 40 (1988): 34.

${ }^{9}$ Sarah Farmer, "Going Visual: Holocaust Representation and Method," American Historical Review 115, no. 1 (2010): 116.
} 
In a review of image use in historical journals from 2000-2009, Valerie Harris and Peter

Hepburn note that "use of images has remained at a relatively consistent level year to year."10

Rather than considering photographs as documents capable of investigation and analysis,

historians have used them primarily as illustrations. According to Hayden White, historians have not "exploited the possibilities of using images as a principal medium of discursive

representation, using verbal commentary only diacritically, that is to say, to direct attention to, specify, and emphasize a meaning conveyable by visual means alone." 11

In the library and information science (LIS) discipline, empirical investigations of visual information use have been similarly scarce. In fact, the phenomenon of visual information (including visual methodologies) has received scant attention overall in LIS, though there is indication that is changing. ${ }^{12}$ Joan Beaudoin explains:

Due to the visual nature of images, aspects surrounding use are markedly different than those of textual materials. The library and information science community has only recently begun to address visual information, and while there have been many successful forays into discerning the phenomena surrounding image retrieval, research to date has failed to adequately address image users' needs and how or why images are being used. ${ }^{13}$

\footnotetext{
${ }^{10}$ Valerie Harris and Peter Hepburn, "Trends in Image Use by Historians and the Implications for Librarians and Archivists," College and Research Libraries 74, no. 3 (2013): 276.

${ }^{11}$ Hayden White, "Historiography and Historiphoty," The American Historical Review 93, no. 5 (1988): 1194.

${ }^{12}$ See Jenna Hartel, and Leslie Thomson. "Visual Approaches and Photography for the Study of Immediate Information Space." Journal of the American Society for Information Science and Technology 62, no. 11 (2011): 111; Jamie Snyder, "Image-Enabled Discourse: Investigating the Creation of Visual Information as Communicative Practice," (PhD diss, Syracuse University, 2012).

${ }^{13}$ Joan E. Beaudoin, "A Framework of Image Use among Archaeologists, Architects, Art Historians and Artists," Journal of Documentation 70, no. 1 (2013): 37.
} 


\subsection{Statement of the Problem}

Digitization of primary sources presents both practical and hermeneutic challenges for historians doing archival research in the $21^{\text {st }}$ century. Navigating digital archival research environments to find and use sources requires competent fluency at varying levels of the interaction, including: interfaces, digitization quality, overall orientation to the collection, and domain-specific heuristics. ${ }^{14}$ As spaces of knowledge production, digital archival research environments must support interpretive and evaluative practices in the midst of numerous factors.

However, limited work has been conducted in LIS to explore how individuals encounter and make sense of digital information. ${ }^{15}$ Empirical research in digital environments tends to focus on single components of the interaction as they relate to specific aspects of information behavior, or to conceptualize information use as the successful fulfillment of stated information needs. Yet user information needs can shift depending on emergent contextual factors. Examining tasks that motivate image selection or identifying relevance criteria employed by users can thus only offer a partial view into the user experience in the specific information space. These perspectives do not attend to the influence that multiple ecological factors (e.g, interface, work context, task, user, image quality) may have on user interactions with materials.

\footnotetext{
${ }^{14}$ Elizabeth Yakel and Deborah Torres conceptualize some of these dimensions as forms of "archival intelligence," an advanced level of expertise in navigating and using archives. See: Elizabeth Yakel and Deborah Torres, "AI: Archival Intelligence and User Expertise," The American Archivist 66, no. 1 (2003): 51-78.

15 Two notable exceptions are: Marcia Bates, "The Cascade of Interactions in the Digital Library Interface," Information Processing and Management 38, no. 3, 2003; Christopher A. Lee, "Digital Curation as Communication Mediation," in Handbook of Technical Communication, eds. Alexander Mehler, Laurent Romary, and Dafydd Gibbon (Berlin: Mouton De Gruyter, 2012), 507-530.
} 


\subsection{Purpose of the Study and Research Questions}

The purpose of this research was to explore the experiences of historians using digitized photographs as forms of evidence. I chose an embedded case study approach to examine how and why historians are using digitized photographs in their scholarly activities. I defined two units of analysis. The main unit of analysis was identified as: "a historian's experiences using digitized photographs as evidence." An embedded unit of analysis, or subunit, was defined as " $a$ historian's specific use of a digitized photograph as evidence in research or instruction."

I employed three types of purposive sampling strategies to recruit fifteen self-identified historians. I conducted a semi-structured interview lasting approximately 1.5 hours with each participant. I used photo-elicitation interviewing and concurrent verbal probing techniques to elicit participants' descriptions of their experiences selecting, interpreting, evaluating, and using images. I also gathered supplementary materials related to how each participant used images as historical evidence in teaching and instructional capacities. Collected documents included conference presentations, teaching syllabus, dissertation chapters, journal articles, and monographs. I used thematic analysis to surface descriptive themes and thematic synthesis to generate analytical themes within and across cases.

The central research question that guided this study is:

$R Q 1$ : What are the experiences of historians using digitized archival photographs as evidence in their scholarly pursuits?

Two additional sub-research questions guided this study:

SubRQ1: How are historians using digitized archival photographs as evidence in research and instructional activities? 
SubRQ2: Why do historians choose to use (or not to use) digitized archival photographs as evidence in scholarly pursuits?

A holistic approach exploring historians' information experiences permitted me to see how and why historians were using images as evidence in the construction of arguments. As a framing device, the notion of information experiences proved useful for revealing various sociomaterial factors that matter to them as they decide to use (or not use) images. Attending to their interactions showed what made their experiences meaningful, or "the difference that makes a difference. ${ }^{\prime 16}$

\footnotetext{
${ }^{16}$ This expression is commonly attributed to Gregory Bateson, from his text Steps to an Ecology of Mind (Chicago: University of Chicago Press, 1972). It is important to note here that the theoretical foundations of my research approach suggest thinking about information as an event, rather than as a stimulus for the difference to which Bateson refers.
} 


\section{CHAPTER 2: LITERATURE REVIEW}

This literature review describes five topical areas related to my research questions. First, I describe humanities scholars' research practices, historians' information behavior in archival settings, and the scholarly impact of digital collections. Next, I trace the history of collecting and describing photographic materials in archival institutions, including a discussion of current descriptive standards for digital image collections. I also consider how varying levels of description impact access to archival materials. In my third section, I focus specifically on LIS approaches to the empirical study of image collection use. My fourth section describes concepts of evidence in modern historiography, moving into an overview of how historians have theorized the use of photographs. I also provide a discussion of three case studies that use photographs to make scholarly arguments. Finally, I explore the socio-material complexities of understanding visual information use in digital environments. I review a series of theoretical frameworks and methodological approaches for conceptualizing information use in digital environments as an emergent experience.

\subsection{Information Practices of Historian Scholars Using Primary Source Materials}

More than twenty years ago, in a user study on researchers in women's history, Diane Beattie lamented that archivists had done very little research on "how users actually locate archival materials, and therefore have no way of measuring the usefulness of current descriptive systems." 17 This continues to be the case today. Studies exploring the information behavior of

\footnotetext{
${ }^{17}$ Diane Beattie, “An Archival User Study: Researchers in the Field of Women's History,”Archivaria 29 (1989): 43.
} 
historians in archives were undertaken nearly a decade ago, ${ }^{18}$ in the early stages of archival digitization efforts. Empirical work that examines historians' use of digitized primary source materials is either limited to citation analysis ${ }^{19}$ or lumped into a wider discussion of how humanities scholars use electronic resources.

This section reviews the literature in the following areas: (1) the larger context of humanities scholars' research practices, including information-seeking behavior; (2) how historians search for and use primary source materials in archival environments; and (3) assessing the scholarly impact of digital collections.

\subsubsection{Research practices of humanities scholars}

The development of effective scholarly infrastructures depends on understanding the evolving information behaviors and research practices of scholars. ${ }^{20}$ Humanities scholars' needs may range from broad exploratory searches to in-depth examination and analysis of source documents. In an influential study from 1982, Sue Stone calls for an exploration of how humanities scholars use the information they acquire. ${ }^{21}$ In the current scholarly climate, such questions become more pressing as we begin to understand and assess how digital tools and

\footnotetext{
${ }^{18}$ Helen Tibbo, "Primarily History in America: How US Historians Search for Primary Materials at the Dawn of the Digital Age," The American Archivist 66, no. 1 (2003): 9-50; Ian Anderson, "Are You Being Served? Historians and the Search for Primary Sources," Archivaria 58 (2004): 88-129; Wendy Duff and Catherine Johnson, Accidentally Found on Purpose: Information-Seeking Behavior of Historians in Archives," Library Quarterly 72, no. 4 (2002): 472-496; Wendy Duff, Barbara Craig, and Joan Cherry, "Finding and Using Archival Resources: A Cross-Canada Survey of Historians Studying Canadian History,” Archivaria 58 (2004): 51-80.

${ }^{19}$ Donghee Sinn, "Impact of Digital Archival Collections on Historical Research," Journal of the American Society for Information Science and Technology 63, no. 8 (2012): 1521-1537. Sinn evaluates the impact of digital archival collection use by historians through the measurement of citations in academic history journals. While useful for quantifying usage statistics, such an approach does not shed light on the information behavior of historians working with digitized archival materials.

${ }^{20}$ Christine Borgman, Scholarship in the Digital Age: Information, Infrastructure and the Internet (Cambridge: MIT Press, 2007), 262.

${ }^{21}$ Sue Stone, “Humanities Scholars: Information Needs and Uses,” Journal of Documentation 38, no. 4 (1982): 297.
} 
technologies have impacted workflow and information behavior. What are the information needs and uses of humanities scholars? How do needs and uses change when searching for and using digital sources? What structures are needed to support and enable scholarly inquiry?

While the literature on humanities scholars' research practices is rich and varied, there are emergent themes that help shed light on their information needs and use. One highly touted perspective is that humanities scholars work independently. ${ }^{22}$ Humanities scholars tend to perform information-seeking tasks themselves rather than delegate to others, as such activities are seen as paramount to their interpretative abilities. ${ }^{23}$

At the same time, a strong sense of collaboration among peers emerges from the literature, particularly in the online realm. Scholarly Work in the Humanities and the Evolving Information Environment, a 2002 report on changing humanities research practices, notes the importance of the "grapevine" as "crucial for supplying references to recent books or articles that might not yet be indexed or cited." 24 Access to online tools such as email, mailing lists, blogs, and wikis can facilitate collaboration and communication among scholars. ${ }^{25}$ The presence of an invisible college, or an informal network of colleagues, enables researchers with limited time to "opt for those techniques that have the highest reward-cost ratio."26

\footnotetext{
22 Stone, "Humanities Scholars: Information Needs and Uses"; American Council of Learned Societies, Our Cultural Commonwealth: The Report of the American Council of Learned Societies Commission on Cyberinfrastructure for the Humanities and Social Sciences (New York: American Council of Learned Societies and Andrew W. Mellon Foundation, 2006).

${ }^{23}$ Stone, "Humanities Scholars: Information Needs and Uses," 294-295.

${ }^{24}$ William Brockman, Laura Neumann, Carole Palmer, and Tonyia Tidline, Scholarly Work in the Humanities and the Evolving Information Environment (Washington, DC: Digital Library Federation and Council on Library and Information Resources, 2002), 11.

${ }^{25}$ Carole Palmer and Laura Neumann, "The Information Work of Interdisciplinary Humanities Scholars: Exploration and Translation," Library Quarterly 72, no.1 (2002): 85-117.

${ }^{26}$ Stephen Stoan, "Research and Information Retrieval among Academic Researchers," Library Trends 39, no. 3 (Winter 1991): 248.
} 
Research pursuits are characterized by divergent and unpredictable routes; methods typically involve "tracing intellectual paths, 'excavating' textual references from documents, and item-by-item review of artifacts held in relevant archival collections." ${ }^{, 27}$ Consequently, browsing is a particularly instrumental function in scholarly workflows. Scholars perform interpretation through the critical practices of reading, browsing, and annotating. They produce "extensive marginal notes, annotating photocopies or personal copies or attaching adhesive notes to a text. ${ }^{28}$

Humanities scholars tend to use a wide array of primary and secondary source materials. ${ }^{29}$ Consulting related works (often called "footnote chaining") is essential. Secondary materials are often consulted for background information or to gain awareness of current research in a field. A critical function of humanities scholarship involves selecting and structuring together meaningful groups of materials to enable interpretation. Authenticity and reliability of materials are the hallmark of trustworthy humanities research endeavors. Even among the earliest user studies, humanist scholars express a strong preference for having access to original documents. ${ }^{30}$

\subsubsection{Information behavior of historians in archival settings}

Historical research in archives is a multi-stage, iterative process. Historians may use a "path-breaking," broad approach to research wherein they propose new ways of looking at old problems, or they may opt for a "micro-historic" narrow approach, in which they examine or

\footnotetext{
${ }^{27}$ Neumann and Palmer, “The Information Work of Interdisciplinary Humanities Scholars,” 98.

${ }^{28}$ Brockman, Neumann, Palmer, and Tidline, Scholarly Work in the Humanities, 7.

${ }^{29}$ Karl Weintraub, “The Humanistic Scholar and the Library,” The Library Quarterly 50, no. 1 (1980): 22-39.

${ }^{30}$ Carole Palmer, "Thematic Research Collections," in A Companion to Digital Humanities (Oxford: Blackwell Publishing, 2004), 348-65; Stone, "Humanities Scholars: Information Needs and Uses," 296.
} 
document a specific community of interest or problem. ${ }^{31}$ Research activities can go on concurrently and may span multiple research projects. Historians often begin their research in archives by orienting themselves to collections; as they build on contextual knowledge and acquire relevant material they further refine and develop their information needs. ${ }^{32}$ Charles Cole's investigation of forty-five history doctoral students formulating their theses finds two essential components at work for students processing information: the picture and the jigsaw. As Cole explains, "The jigsaw appears to be the Ph.D. history student's conceptual thesis and the picture is the background 'data' from which the jigsaw emerges." ${ }^{\text {"33 }}$ Cole's research shows how the cognitive aspects of information processing can impact knowledge formulation in historical research.

Research practices within archival settings appear to be changing due to the impact of modern digital technologies. In addition to the fact that many archives provide online access to digitized versions of primary source materials, personal capture devices are now permitted in many reading rooms. The use of digital cameras, flatbed scanners, and laptops enables historians to personally document archival materials of interest. A recent survey investigating the changing research practices of historians found widespread use of digital cameras and scanning equipment to capture primary source materials, claiming it was "perhaps the single most significant shift in research practices among historians, and one with as-yet largely unrecognized implications for

\footnotetext{
${ }^{31}$ Donald Case, "The Collection and Use of Information by Some American Historians: A Study of Motives and Methods," Library Quarterly 61, no. 1 (1991): 71.

${ }^{32}$ Wendy Duff and Catherine Johnson, “Accidentally Found on Purpose: Information-Seeking Behavior of Historians in Archives," Library Quarterly 72, no. 4 (2002): 492.

${ }^{33}$ Charles Cole, "Information Acquisition in History Ph.D. Students: Inferencing and the Formation of Knowledge Structures," Library Quarterly 68, no. 1 (1998): 42.
} 
the work of historical research and its support." ${ }^{34}$ Numerous publications on best practices for digital capture activities over the last decade suggest that new kinds of workflows have begun to emerge in archival settings. ${ }^{35}$

Historians typically consult a large number of institutions during the archival research process. ${ }^{36}$ Archival institutions may include public or university libraries, academic special collections/repositories, state or local historical societies, museums, and state or government archives. Not surprisingly, the types of institutions historians consult may depend significantly on their specific topics of historical research. In his citation analysis study on social historians, Frederic Miller finds a low use of state and local archives, calling them "the most underutilized resources in the nation's archival system.”37 Meanwhile, Wendy Duff, Barbara Craig and Joan Cherry, also looking at social historians, find a high use $(90 \%)$ of provincial archives. ${ }^{38}$ Their specific focus on Canadian history rather than an overall focus on social history topics may explain the high use.

Similarly, the types of archival materials used by historians often relates to their topic of research. Historians working on biographical research, for example, tend to use "collection-

\footnotetext{
${ }^{34}$ Jennifer Rumer and Roger C. Schonfeld, Supporting the Changing Research Practices of Historians, Final Report from ITHAKA $S+R$ (2012), 11.

${ }^{35}$ Kirklin Bateman, Sheila Brennan, Douglas Mudd, and Paula Petric, "Taking a Byte Out of the Archives: Making Technology Work For You," Perspectives: Newsletter of the American Historical Association 43, no. 1 (2005); Richard J. Cox, "Machines in the Archives: Technology and the Coming Transformation of Archival Reference," First Monday 12, no. 11 (2007); Lisa Miller, Stephen K. Galbraith, and RLG Working Group, "Capture and Release": Digital Cameras in the Reading Room (Dublin, OH: OCLC Research, 2010).

${ }^{36}$ Case, "The Collection and Use of Information by Some American Historians: A Study of Motives and Methods," 74.

${ }^{37}$ Frederic Miller, “Use, Appraisal, and Research: A Case Study of Social History,” The American Archivist 49, no. 4 (1981): 381.

${ }^{38}$ Wendy Duff, Barbara Craig, and Joan Cherry, "Finding and Using Archival Resources: A Cross-Canada Survey of Historians Studying Canadian History," Archivaria, 58 (2004): 59.
} 
oriented tools" like finding aids. ${ }^{39}$ In some cases, limited archival holdings on subjects may force historians to use non-traditional types of sources. Diane Beattie's study on Canadian historians studying women's history shows frequent use of photographs and oral history recordings; she attributes this to the dearth of archival materials related to women's history. ${ }^{40}$

The literature does not provide conclusive evidence on historians' preferred search and retrieval strategies in archival settings. When asked about the techniques most often used to locate primary sources, historians describe the consultation of published finding aids, ${ }^{41}$ communication with an archivist, ${ }^{42}$ and following leads or citations found in printed books. ${ }^{43}$ In part, the discrepancies could be attributed to different goals in each research study. For example, Tibbo and Anderson are concerned with the impact of digital tools on historians' search behaviors. Thus, they categorize each search method as either print or electronic. Other semantic differences complicate effective comparisons across studies. What historians might consider to be the most useful methods for locating materials does not necessarily translate into the most frequently used. In Beattie's study, historians claim that they most frequently consult archivists in their search for materials but cite finding aids to be the most useful in their search. ${ }^{44}$

Another complicating factor is a tendency in research studies to collapse the individual tasks associated with information search, retrieval, and use into a single behavior (often,

\footnotetext{
39 Tibbo, "Primarily History in America," 21.

${ }^{40}$ Beattie, “An Archival User Study,” 42.

${ }^{41}$ Margaret Stieg-Dalton and Laurie Charnigo, "Historians and Their Information Sources," College and Research Libraries 65, no. 4 (2004): 407; Wendy Duff, Barbara Craig, and Joan Cherry, "Historians Use of Archival Sources: Promises and Pitfalls of the Digital Age," The Public Historian 26, no. 2 (2004): 14.

${ }^{42}$ Beattie, "An Archival User Study," 43.

${ }^{43}$ Anderson, “Are You Being Served?" 95; Tibbo, "Primarily History in America," 20.

${ }^{44}$ Beattie, “An Archival User Study,” 44.
} 
"information-seeking") that fails to account for multiple steps in the research process. A historian may begin his or her search by talking to an archivist, who then may point him or her to an electronic database where he or she might retrieve a source. Finally, many studies do not account for the fact that the methods historians use to search for unknown materials may be quite different from how they search for known materials. Duff's characterization of a historian who "orients" herself in archives would suggest that search behavior might differ according to familiarity. ${ }^{45}$

\subsubsection{Scholarly use of digitized collections}

Overall, the emergence of digitized primary source collections is generally portrayed as positive for humanities scholarship. Some of the major advantages of digital collections include: the portability of digital formats, fast access, and better searching techniques. ${ }^{46}$ Access to primary source materials is especially beneficial for scholars; preliminary browsing of digital library collections can save time and money for scholars in judging the relevance of materials to their research. ${ }^{47}$

At the same time, scholars also acknowledge challenges that can arise from working with digitized primary source materials in online environments. The LAIRAH (Log Analysis of Internet Resources in the Arts and Humanities) project, a fifteen-month inquiry into the factors that determine use (and neglect) of digital materials in the arts and humanities, found that users require a tremendous amount of information to discern both context and relevance. In the

\footnotetext{
${ }^{45}$ Duff and Johnson, “Accidentally Found on Purpose,” 472-496.

${ }^{46}$ Weiyin Hong, James Thong, Wai-man Wong, and Kar-yan Tam, "Determinants of User Acceptance of Digital Libraries: An Empirical Examination of Individual Differences and System Characteristics," Journal of

Management Information Systems 18, vol. 3 (2002): 99.

${ }^{47}$ Jane Segal and Lisa Spiro, “The Impact of Digital Resources on Humanities Scholarship,” Rice University, 2007.
} 
absence of a physical browsing space, the authors note it can be difficult to comprehend both the coverage and extensiveness of the resource. They explain, "Scholars can browse a library shelf or journal issues and quickly determine the approximate extent of the resources available, and thus be sure that they do not miss anything important, but this is much more difficult in the case of digital resources." 48

The general lack of empirical data about digital collection usage is problematic. One study on digital resource use, conducted in 2006 at the University of California at Berkley, reports, “The 'build it and they will come' approach has resulted in a widely acknowledged supply-driven movement," but it is not yet clear just how much scholars are using available digital collections. The authors assert the importance of studying use and reuse, particularly to assuage the fears of funding agencies that are "concerned about the low level of use of available digital resources among the teaching faculty of our institutions." 49

The research that has been conducted on scholarly use of digital collections tends to focus primarily on quantifying use through transaction $\log$ analysis or citation analysis, rather than exploring scholarly use of, and satisfaction with, digital resources. While the former is helpful for uncovering usage patterns, it is not appropriate for understanding experiential aspects of information use. As Laura Sheble and Barbara Wildemuth point out, transaction logs "cannot tell us anything about the users' cognitive or affective responses during the system interaction."50

\footnotetext{
${ }^{48}$ Claire Warwick, Melissa Terras, Paul Huntington, and Nikoleta Pappa, "If You Build It Will They Come? The LAIRAH Study: Quantifying the Use of Online Resources in the Arts and Humanities through Statistical Analysis of User Log Data," Literary and Linguistic Computing 23, no. 1 (2008): 99.

${ }^{49}$ Diane Harley, Jonathan Henke, Shannon Lawrence, Ian Miller, Irene Perciali, and David Nasatir, Use and Users of Digital Resources: A Focus on Undergraduate Education in the Humanities and Social Sciences (UC Berkeley: Center for Studies in Higher Education, 2006): 2-1.http://escholarship.org/uc/item/8c43w24h.

${ }^{50}$ Laura Sheble and Barbara Wildemuth, "Transaction Logs," in Applications of Social Research Methods to Questions in Information and Library Science, ed. Barbara Wildemuth (Westport, CT: Libraries Unlimited, 2009), 174.
} 
Citation analysis is only partially helpful as an indicator of information use; scholars may consult a number of primary source materials in the course of their research but not necessarily cite them.

Particular challenges lie in building effective infrastructure for historians to use digitized archival materials. In order to provide a seamless experience for historical research, infrastructure should support both historical practices and technological efforts. In their report summarizing the history of computer-aided historical research, Onno Boonstra, Leen Breure and Peter Doorn explain the importance and the intricacies of achieving this delicate balance:

Better infrastructure is needed in order to guarantee a transfer of results from the methodological and technical level to the daily practice of historical research. On the contrary, denying these challenges and opportunities will, in the long run, segregate the study of history from the technical capabilities currently being developed in the information society and will turn 'the computer' into an awkward tool with limited use and usability for historians. ${ }^{51}$

How do the archivist and the historian, each possessing different domain expertise, effectively work together? What role does the archivist play in constructing authentic digital environments? How should historical materials be displayed and presented so that context is not lost? These are pressing questions. Continued research on the evolving information needs of academic historians can aid the construction of successful archival research environments.

\subsection{Describing Photographs in Archival Collections: History, Methods and Implications}

This section discusses the historical context, theoretical basis, and practical concerns related to the description of and access to photographic materials in archival institutions. First, I provide an overview of the collecting of photographic materials by American institutions. Next, I briefly summarize the development of archival and library standards for cataloging visual

\footnotetext{
51 Onno Boonstra, Leen Breure and Peter Doorn, "Past, Present, and Future of Historical Information Science," Historical Social Research 29 (Amsterdam: Netherlands Institute for Scientific Information, 2004 ): 91.
} 
materials, moving into a review of current metadata standards for describing photographs and image collections overall. Finally, I explore how archivists propose describing photographs and investigate critiques which claim that a failure to develop and implement appropriate descriptive models prevents adequate access to, and use of, photographic materials.

\subsubsection{Institutions and photographic materials}

Institutions in the United States have long recognized the importance of acquiring and collecting photographic materials as documentation. One of the earliest and most widespread uses of photography in an institutional context was the capture and recording of war activities. Writing in the American Archivist in 1958, Joe Thomas describes an 1860 U.S. expedition to Japan that used daguerreotypes to document governmental activities wherein "an agency of the United States Government in connection with the transaction of public business had consciously created photographic records that were appropriate for preservation as evidence of its operations or as the embodiment of valuable information." 52 In 1874, the photographic collection of noted civil war photographer Matthew Brady was purchased by the United States Department of War. In fact, the Comptroller went on to sanction the publication of the photographs as "official records" of the war, claiming that "the photographic views of the war, showing battlefields, military divisions, fortifications, etc. are among the most authentic and valuable records of the rebellion." ${ }^{, 3}$ Brady's collection was transferred to the War Department's library in 1888.

Cultural heritage institutions were also readily acknowledging the importance of photographs as documentation. The Library of Congress (LOC) added photographs to the list of

\footnotetext{
52 Joe D. Thomas, "Photographic Archives," The American Archivist 21, no.4 (1958): 420.

${ }^{53}$ United States, David Fitzgerald, and A. W. Greely, List of the Photographs and Photographic Negatives Relating to the War for the Union, Now in the War Department Library (Washington DC: Government Printing Office, 1897), 7. https://archive.org/details/listofphotograph00unit.
} 
copyrighted items required for deposit in $1865 .^{54}$ The Metropolitan Museum of Art reported a nearly 14,000-item photograph collection in 1906 (with 12,000 items added that year). ${ }^{55}$ In 1914, due to increased public requests, the Circulation Department at the New York Public Library began gathering materials for its "Picture Collection"; by the close of that year, the collection amassed nearly 18,000 items. ${ }^{56}$ The U.S. National Archives began collecting photographs from federal agencies shortly after its inception in 1934 and even housed a separate division called Photographic Archives. ${ }^{57}$ In 1944, the LOC's newly renamed Print and Photographs Division acquired more than one-hundred thousand photographs and negatives as part of the Farm Security Administration-Office of War Information collection, which documented the life of American farmers between 1935 and 1943. The collection has gone on to become one of the most iconic in American history.

\subsubsection{Descriptive approaches to photographic print materials}

Institutions establishing visual collections in the late nineteenth and early twentieth centuries had to develop their own standards for cataloging materials. An early and influential publication which provided guidance is John Cotton Dana's The Picture Collection, published in 1910. Dana oversaw the creation of the first so-called picture collection at the Denver Public Library in 1888; he went on to become the chief librarian at the Newark Public Library in 1902. The Picture Collection advised cataloging materials by alphabetized subject headings.

\footnotetext{
${ }^{54}$ Renata V. Shaw, A Century of Photographs, 1846-1946 (Washington DC: The Library of Congress, 1980), Introduction.

${ }^{55}$ Charles Ammi Cutter, Library Journal 32 (New Jersey: R.R. Bowker Company, 1907), 235.

${ }^{56}$ Anthony T. Troncale, "Worth Beyond Words: Romana Javitz and the New York Public Library's Picture Collection," New York Public Library Website. http://www.nypl.org/locations/tid/45/node/62019.

${ }^{57}$ Barbara Lewis Burger, Guide to the Holdings of the Still Picture Branch of the National Archives and Records Administration (Washington, DC: National Archives and Records Administration, 1990), Introduction section, para 3. http://www.archives.gov/research/guides/still-pictures-guide.html.
} 
Our experience has led us always to the same conclusion; that no arrangement is as good as an alphabetical one by subject because the pictures under this arrangement need no catalog and are self-indexing. They are filed vertically, like cards in a catalog, and the names of the subjects under which they are arranged are written near the tops of the mounts. They can thus be easily consulted and pictures on any desired subject easily found." $" 58$

The book specifies nearly 2000 subject headings for use in classification. Dana goes on to note that while he originally intended to use American Library Association subject headings, he instead chose to base headings on "local conditions and observation of the use of the Picture Collection by borrowers." 59

Card catalog subject access, however, presented a problem for finding materials: patron requests had to match already-conceptualized subject headings. As Anthony Troncale explains, Dana's particular subject heading classifications presented barriers to access:

In his idiosyncratic filing system, for example, one would have to look under " $F$ " for "Forms of Land and Water" to find "Niagara Falls," a situation reflecting nineteenthcentury bibliographical practice and hierarchical mind-set. A reassessment of subject headings would be needed to reflect the fast-changing styles and fashions of the machine age and their new nomenclatures. ${ }^{60}$

The limitations of the subject classification approach were recognized by the New York Public Library's Romana Javitz, who began working at the Picture Collection in 1924. She employed a myriad of creative approaches to improve subject access to visual materials. To overcome a language barrier, she instituted a policy in 1931 requiring all patrons to draw or describe picture requests on call slips. She also used Works Progress Administration (WPA) artist-trained workers to provide subject descriptions for an ever-increasing number of visual items acquired

\footnotetext{
${ }^{58}$ John Cotton Dana, The Picture Collection (Woodstock, Vermont: Elm Tree Press, 1910), 26.

${ }^{59}$ Ibid, 28.

${ }^{60}$ Troncale, "Worth Beyond Words," para. 7.
} 
by the Picture Collection. Javitz introduced the use of new schemes (based on regions, styles, types and year) to provide even greater flexibility in access. ${ }^{61}$

The Minnesota Public Library adopted Dana's approach for use in "Minneapolisiana," the historical photograph collection they created in 1940. The subject index approach provided library visitors the ability to browse through a multitude of images. As Ruth Thompson, the head of the Minnesota Historical Collection, pointed out: "Often patrons do not ask for a specific picture, but need the inspiration which comes from looking over different folders on a variety of subjects." 62

The Library of Congress began using subject categorization to organize photographic collections in 1943, following the appointment of its first curator of photography. Photographs were initially sorted according to subject categories and then grouped into lots - or "batches of pictures which fall into logical groups by virtue of their creator or donor, the overall title of a picture story, or the unifying subject matter." ${ }^{93}$ The next ten years saw the acquisition of many important collections, including photographic documentation of the Crimean War, Civil War, and Nazi Germany. During the 1960s, the Library set forth new guidelines to distinguish between artistic and documentary photographic collections; the former were arranged and grouped as "Master Photographs." "64

The development of the Machine-Readable Cataloging (MARC) standard in the mid1960s at the LOC was a watershed moment for librarians and archivists. MARC enabled the

\footnotetext{
${ }^{61}$ Ibid, paras. 13-18.

${ }^{62}$ Ruth Thompson, "The Collection and Preservation of Local Historical Pictures in the Minneapolis Public Library," The American Archivist 9, no. 4 (1946): 223.

${ }^{63}$ Shaw, 2.

${ }^{64}$ Ibid, 3.
} 
storage of machine-readable, exchangeable bibliographic information about institutional holdings. ${ }^{65}$ A companion to MARC, the publication of The Anglo-American Cataloging Rules (AACR) in 1967 provided guidance for institutions in creating and describing bibliographic information in a standardized format. The second edition of the book, AACR2, was published in 1978 and served as the standard for use in describing library materials for nearly thirty-five years. In March 2013, the Library of Congress introduced a new cataloging standard, the Resource Description and Access Framework (RDA). ${ }^{66}$

The widespread adoption and use of MARC and AACR2 by librarians during the 1970s also revealed limitations in their structures. Both AACR2 and MARC were originally designed to accommodate book formats; meanwhile, institutions had been collecting many different types of materials in varying formats. In 1978, an Independent Research Libraries Association (IRLA) Ad-Hoc committee formed to evaluate how well the MARC format met the needs of catalog users. In particular, the committee sought to investigate whether MARC accurately represented audiovisual (AV) materials. On the one hand, the current MARC standard stipulated that subject headings be as specific as possible to enable better access points. However, most users of AV materials required the ability to search and use materials in a broad, interdisciplinary manner. ${ }^{67}$ The IRLA committee report thus proposed the addition of two auxiliary MARC fields, "genre" and "publishing/physical characteristics" to better describe, and provide access to, specialized materials. The committee also recommended the creation of a controlled vocabulary thesaurus for indexing terms.

\footnotetext{
${ }^{65}$ Walt Crawford, MARC for Library Use (Boston, MA: GK Hall, 1989), 1.

${ }^{66}$ See W3C, “The Resource Description Framework Homepage,” http://www.w3.org/RDF/.

${ }^{67}$ Paul Graham, “Current Developments in Audiovisual Cataloging." Library Trends 34, no. 1 (1985): 59.
} 
In 1980, the Thesaurus for Graphic Materials I (TGM 1) was created at the Library of Congress to provide a controlled vocabulary for describing the subjects of pictorial works. TGM 1 originated from the work of the Prints and Photographs' Division cataloger Elizabeth Betz Parker, who culled together subject headings used to describe division holdings from the previous fifty years. ${ }^{68}$ The subject terms are collection-driven and thus have grown significantly since inception; new terms can be proposed through a submission process on the LOC's website. A companion volume, Thesaurus for Graphic Materials 2 (TGM 2), appeared in 1986. TGM2 aimed to enable additional access points to visual collections by providing fixed vocabulary terms for describing both genre and physical format characteristics. ${ }^{69}$ In 2007, TGM1 and TGM2 were combined into a single thesaurus, Library of Congress Thesaurus for Graphic Materials (LCTGM).

Betz Parker also developed Graphic Materials: Rules for Describing Original Items and Historical Collections in 1982. The manual was created in an attempt to unite archival principles with library cataloging approaches, for use in describing graphic materials in standardized formats. In her introduction to the manual, Betz Parker explains the impetus behind the creation of separate cataloging guidelines for such materials:

The concepts of "chief source of information" and "prescribed sources" used in traditional book cataloging has been redefined here for original and historical graphic materials because they often have little or no text to transcribe. The major reason for documenting graphics is to provide the researcher with as complete an identification of the material as possible. This is done by translating the visual information into a verbal description of the material's physical nature and image content. ${ }^{70}$

\footnotetext{
${ }^{68}$ Arden Alexander and Tracy Meehleib, "The Thesaurus for Graphic Materials: Its History, Use, and Future," Cataloging \& Classification Quarterly 31, no.3-4 (2001): 191.

${ }^{69}$ For a helpful discussion of the implications and importance of describing the physical characteristics of archival materials, see: Helena Zinkham, Patricia D. Cloud, and Hope Mayo, "Providing Access by Form of Material, Genre, and Physical Characteristics: Benefits and Techniques," The American Archivist 52 (Summer 1989): 315.

${ }^{70}$ Elisabeth Betz-Parker, Graphic Materials: Rules for Describing Original Items and Historical Collections (Washington, DC: Library of Congress, 1982), 4-5.
} 
Betz Parker goes on to acknowledge the equal importance of cataloging items at both the item and the collection-level, noting that the latter is essential for understanding and preserving the context of the former.

The Arts and Architecture Thesaurus (AAT) was also developed during the early 1980s, from joint efforts in the library and art/architectural history communities. It has been maintained by the Getty Research Institute since 1983, and currently contains more than 34,000 concepts and 130,000 terms. Concepts are linked to related terms and arranged in a hierarchal fashion, from general to specific. Seven facets are used to enable access points at multiple levels of description: associated concepts, physical attributes, styles and periods, agents, activities, materials, and objects. ${ }^{71}$ In her comparison between LCGTM and AAT, Greenberg notes the latter is a "very specialized source with terminology specifically for the built environment, furnishings and equipment, and visual and verbal communication" while the former provides greater use for a broader audience. ${ }^{72}$

During the mid to late 1980s, a number of theoretical approaches for describing visual materials emerged from the LIS community in an attempt to counter problems with access. Sara Shatford-Layne's work at the Library of Congress represents one of the first attempts to put forth a framework to deal with the inherent complexities of visual materials. Drawing on the work of art historian Erwin Panofksy, as well as approaches first articulated by Ranganathan's Colon Classification, and later on at the Bibliotheque Nationale de France (BnF), Shatford-Layne‘s

\footnotetext{
${ }^{71}$ For an in-depth exploration of AAT's seven facets, see: Michael Joseph, "Information Technology and Access to Visual Images in Printed Books," Visual Resources 11, no.1 (1995): 78-80.

72 Jane Greenberg, "Intellectual Control of Visual Archives: A Comparison between the Art and Architecture Thesaurus and the Library of Congress Thesaurus for Graphic Materials," Cataloging \& Classification Quarterly 16, no. 1 (1993): 94.
} 
framework identifies and classifies the kinds of subjects used to describe pictorial works. She writes:

The different facets for the classification of the subjects of pictures may be defined initially as containing the answers to a series of questions asking Who? What? When? and Where? Each of these basic facets may then be subdivided into aspects based on $O f$ in the specific sense, $O f$ in the generic sense, and About $^{73}$

Importantly, Shatford-Layne's approach enables both a specific and generic approach to subject classification of pictures. For example, a photograph of the Sears Tower can be described both as a specific object of interest ("The Sears Tower") and in terms of its generic ofness (“Skyscraper”). Such distinctions are critical in describing visual materials, which can be perceived at multiple levels of description and thus should accommodate varying entry points for access.

As an example of how this plays out in descriptive practice, consider the LOC's Herblock Collection, which contains editorial cartoon drawings by Washington Post cartoonist Herbert L. Block. The collection consists of 14,000 original ink and graphite drawings. One item from the collection, "Well, everything helps," has two vastly different format descriptions: "Editorial cartoons--1930-1940" and "Ink drawings--1930-1940." 74 The former addresses the content of the item while the latter addresses the medium. Enabling both levels of access through description is important because they serve different functions depending on information needs. Art historians, for example, may want to look explicitly at materials of a certain format or medium. Social or cultural historians, however, may want to use to these materials as sources

\footnotetext{
${ }^{73}$ Sara Shatford-Layne, "Analyzing the Subject of a Picture: A Theoretical Approach," Cataloging \& Classification Quarterly 6, no. 3 (March 1986): 48.

${ }^{74}$ Herbert Block, "Well, Everything Helps," cartoon, Library of Congress Prints and Photographs Online Catalog, 1930 or 1931, http://www.loc.gov/pictures/item/00652175.
} 
for examining editorial representations in mainstream media, to better understand popular sentiment at the time.

Shatford-Layne's framework also distinguishes between subject analysis of a medium (e.g., how to describe a painting) and subject analysis of the reproduction of that medium (e.g., how to describe a photograph of that painting). She introduces the concept of the Represented Work as a means for addressing these conceptual differences. A Represented Work is "a particular kind of subject that a picture can have, but that a text cannot. A picture is $O f$ a tangible object in a way that a text, composed of words, can never be." ${ }^{\text {75 }}$ Shatford-Layne goes on to critique traditional cataloging practices, which she claims confuse reproductions with original works in catalog descriptions of materials.

The work of Karen Markey during the mid to late 1980s represents another attempt to theoretically address the complexities of visual material description through the establishment of baseline attributes. ${ }^{76}$ Markey claims that the increasing availability of MARC data is beneficial for expanding access points. Like Shatford-Layne, Markey invokes Panofsky's three levels of meaning to suggest enhanced descriptions for additional points of subject access to visual materials. ${ }^{77}$ Rather than limit description to secondary subject matter (based on the norm, due to traditional design constraints of the card cataloging system), Markey suggests that through the use of MARC data and the training of catalogers, primary subject matter can also be described easily and efficiently to expand access.

\footnotetext{
${ }^{75}$ Shatford-Layne, "Analyzing the Subject of a Picture," 51.

${ }^{76}$ Karen Markey, Subject Access to Visual Resources Collections (Greenwood Press: The University of Michigan, 1986); Karen Markey, “Access to Iconographical Research Collections," Library Trends 37, no. 2 (1988).

${ }^{77}$ The art historian Erwin Panofsky formulates three different kinds of "meaning" which can be experienced in a work of art: primary matter (objects, events, forms); secondary matter (types, themes, concepts); and intrinsic meaning (cultural symptoms, symbols, essential tendencies). See: Erwin Panofsky, Meaning in the Visual Arts (Garden City, NY: Doubleday Anchor Books, 1955), 40-41.
} 
A conceptual framework proposed in the mid-1990s by Corrine Jorgensen examines the types of attributes participants used to describe images while performing three different types of tasks. ${ }^{78}$ The primary focus of her research is to identify useful indexing attributes for end users of image retrieval systems. Her analysis concludes that twelve different types of attributes can be used to describe images (see Figure 1). She then categorizes each of the twelve attributes into three categories: perceptual, interpretive, and reactive. One of Jorgensen's most interesting conclusions is that respondents tend to describe images within a specific context - what she terms "a story" using attributes such as "activity" and "setting.",79

\footnotetext{
${ }^{78}$ Corinne Jorgensen, "Image Attributes: An Investigation" (PhD diss., Syracuse University, 1995); Corinne Jorgensen, "Attributes of Images in Describing Tasks," Information Processing \& Management 34, no. 2/3 (1998).

${ }^{79}$ Jorgensen, “Attributes of Images,” 168.
} 


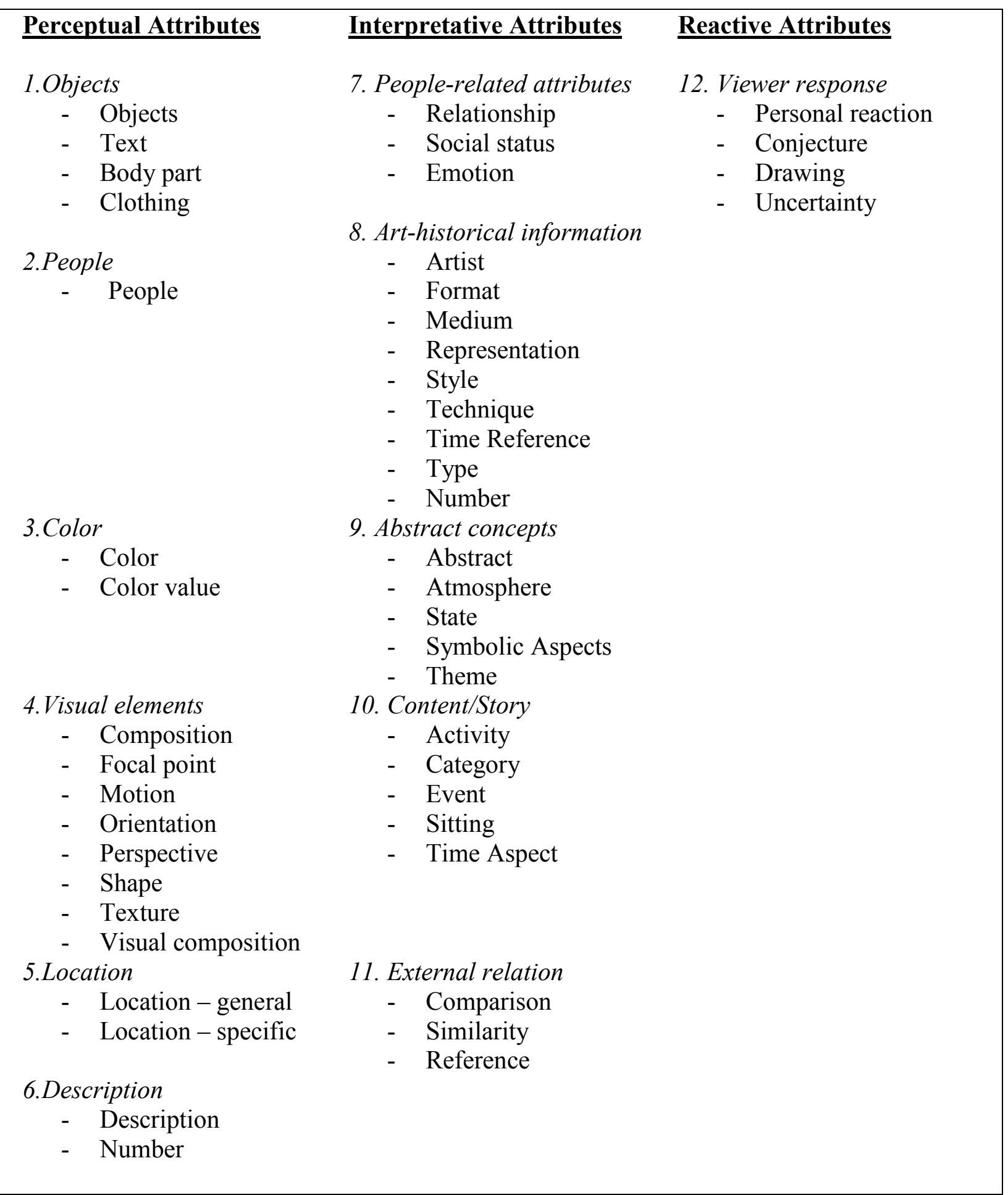

Figure 1. Jorgensen's twelve types of image attributes 


\subsubsection{Descriptive standards for digital image collections}

The growth of digital library projects during the 1990s introduced massive amounts of electronic information for consumption. One of the most pressing needs facing digital libraries was how best to enable resource discovery in this vast landscape. Indeed, a 1995 workshop on digital libraries held by the newly inaugurated Information Infrastructure Technology and Applications (IITA) named "resource discovery" to be one of its critical research items. ${ }^{80}$ In the Strawman Report from the workshop, Hector Garcia-Molina explains, "The challenge is to characterize the information contents (e.g., meta-information) and service capabilities of libraries in a compact and meaningful way." 81 The capture of meta-information, or "metadata" emerged as the primary mechanism for managing digital objects. Metadata acts as a surrogate for digital objects by describing their properties as discrete elements. It can be defined as "data about data that supports the discovery, use, authentication, and administration of information objects." 82 Metadata schemas provide appropriate organizational structures for elements, helping to define and standardize attributes to describe, and enable access to, materials.

The development and use of metadata schemas enables digital libraries to describe digital objects and exchange information about holdings. One of the first widely used metadata schemas adopted for use in digital libraries was the Dublin Core, originating out of an

\footnotetext{
${ }^{80}$ The IITA was a subset of the National Information Infrastructure (NII), a nationwide initiative in the early $1990 \mathrm{~s}$ to support networked infrastructure. One of the IITA's goals was to develop the underlying technology for the NII's efforts. For a brief history of the IITA and its relationship to digital library efforts, see: Stephen M. Griffin, "NSF/DARPA/NASA Digital Libraries Initiative: A Program Manager's Perspective," D-Lib Magazine, July/August 1998, http://www.dlib.org/dlib/july98/07griffin.html.

${ }^{81}$ Hector Garcia-Molina, "Digital Libraries Research Agenda Report: A Strawman Report for the IITA Digital Libraries Workshop,” para. 5, http://dbpubs.stanford.edu:8091/diglib/pub/reports/iita-dlw/appendix2.html.

82 Jane Greenberg, "A Quantitative Categorical Analysis of Metadata Elements in Image-Applicable Metadata Schemas," Journal of the American Society for Information Science and Technology 52, no. 11 (2001): 918.
} 
invitational workshop held in 1995 in Dublin, Ohio. It consists of fifteen core elements deemed essential for basic resource discovery. The Dublin Core standard consists of two levels: qualified or unqualified. Qualified Dublin Core uses qualifiers to more precisely describe elements. For example, the element "date" can be further refined to indicate the type of date (for example, "last modified" or "last published"). Unqualified (or simple) Dublin Core expresses each element without further refinement. ${ }^{83}$ All elements are optional and repeatable, making it a simple, flexible, and extensible schema.

The appropriateness of using the Dublin Core for scholarly image access was explored at an Image Metadata Workshop in 1996. The two-day workshop concluded that text and images had essentially the same discovery requirements for researchers. The group reached consensus that the Dublin Core "affords a foundation for the development of a simple resource description model to support network-based discovery of images." ${ }^{\prime 4}$ Modifications were made to the original schema; for example, the element "description" was separated out from the element "subject" because image specialists felt that the two presented conceptual differences when describing images.

At the same time, the group also recognized several limitations with using the Dublin Core for image collections. The inherent complexities in describing images that had relationships with multiple surrogates were seen as one challenge. Another concern was the Dublin Core's emphasis on discovery, which is only one component of scholarly image use. The group concluded that for metadata to truly support the scholarly research process, different types

\footnotetext{
${ }^{83}$ The Dublin Core Usage Guide provides guidance and information on the Dublin Core element set. See: http://wiki.dublincore.org/index.php/User_Guide.

${ }^{84}$ Stuart Weibel and Eric Miller, "Image Description on the Internet: A Summary of the CNI/OCLC Image Metadata Workshop," D-Lib Magazine, 1997, para. 2, http://www.dlib.org/dlib/january97/oclc/01weibel.html.
} 
of element sets for different functions would need to be developed. Finally, it was acknowledged that the schema did not record significant details about the image digitization process. In their summary of the discussions at the workshop, Weibel and Miller write: "Characteristics of original image capture, while less critical for the casual user, may be of overwhelming importance to the archival or research significance of the image or collection. This sort of information is also, for the most part, irrecoverable if not recorded at the time of capture." 85

Despite these limitations, the schema was hailed as a first step toward establishing baseline metadata elements for the discovery of electronic resources. In fact, analysis on metadata schema usage in digital collections suggests that the Dublin Core has been widely adopted in libraries and archives. In 2007, Ma surveyed 123 American Research Libraries (ARL) members and found that $78 \%$ reported using the Dublin Core to manage digital objects. ${ }^{86}$ Following MARC and Encoded Archival Description (EAD), it was the third most widely used schema. More recently, Park and Tosaka surveyed librarians and catalogers and found that $66 \%$ of institutions used either the qualified or non-qualified version of the Dublin Core. ${ }^{87}$ While the high use of the Dublin Core in cultural heritage institutions is well documented, the extent to which the schema is used specifically to describe images is unknown.

The Visual Resources Association Core (VRA Core) is a descriptive metadata standard designed specifically for visual materials. It was "initially developed to fill an urgent, perceived

\footnotetext{
${ }^{85}$ Ibid, para. 15.

86 Jin Ma, "Metadata in ARL Libraries: A Survey of Metadata Practices," Journal of Library Metadata 9, no. 1-2 (2007): 5 .

${ }^{87}$ Jung-Ran Park and Yuji Tosaka, "Metadata Creation Practices in Digital Repositories and Collections: Schemata, Selection Criteria, and Interoperability," Information Technology \& Libraries 29, no. 3 (2010): 108.
} 
need for a documentation standard to guide the cataloging of image collections." ${ }^{88}$ To formulate the first version of core elements, the VRA surveyed the data elements used in visual resource records in more than sixty institutions. VRA Core 1.0 was released in 1996 and contained twenty-one elements grouped into three different categories of description: object, creator, and subject.

While the VRA Core established the necessary data structures, it did not offer data input guidelines for describing content. An initial assessment of the standard took place in the Vision Project, which examined more than one thousand VRA core records created by catalogers at more than thirty-three institutions. The evaluation results were problematic; as Kessler explains: "There was confusion in the interpretation of the intent of the elements and there was glaring inconsistency in the accumulated data values." 89 The project concluded that a data content standard was needed to guide the successful implementation of VRA Core in institutions. Efforts to produce such a standard culminated in 2006, with the publication of Cataloging Cultural Objects: A Guide to Describing Cultural Objects and their Works (CCO). The CCO operates similarly to AACR2 in that it informs how cataloging records should be created and populated. ${ }^{90}$ The key difference is that $\mathrm{CCO}$ provides guidelines specifically for visual materials for cultural heritage institutions.

The current version of the standard, known as VRA Core 4, describes materials at three different conceptual levels: "work," "image," and "collection." Unlike the Dublin Core, the

\footnotetext{
${ }^{88}$ Visual Resources Association. Core Categories for Visual Resources, Version 4.0, 2007, http://www.vraweb.org/projects/vracore4/index.html.

${ }^{89}$ Ben Kessler, "Encoding Works and Images: The Story behind VRA Core 4.0," VRA Bulletin (2007): 24.

90 To learn more about CCO, see: Visual Resources Association, “Cataloging Cultural Objects,” http://cco.vrafoundation.org/.
} 
VRA Core structure allows for relationships between single entities (unique events or objects constitute a "work") and representations of those entities (described as "images). The flexibility of this approach proves useful for describing the complex relationships among works of art and their surrogates. The VRA Core documentation describes an image thusly:

An image is a visual representation of a work in either whole or part. The representation serves to provide access to the work when the work itself cannot be experienced firsthand. In image collections, such representations typically are found in the form of slides, photographs, and/or digital files. ${ }^{91}$

The most recent version of Core (v.4) introduced an extensible markup language (XML) schema to enable data exchange and interoperability with other data standards. There are currently eighteen elements and twenty-three sub-elements that can be used to describe visual materials.

During the mid-1990s, archivists began developing Encoded Archival Description (EAD), which provides access to machine-readable archival finding aids. ${ }^{92}$ EAD was conceived and formulated in accordance with the principles adopted by the International Council on Archives' for descriptive standards, known as the General International Standard Archival Description, Second Edition (ISAD(G)). ${ }^{93}$ As ISAD(G) states:

The purpose of archival description is to identify and explain the context and content of archival material in order to promote its accessibility. This is achieved by creating accurate and appropriate representations and by organizing them in accordance with predetermined models. Description-related processes may begin at or before records creation and continue throughout the life of the records. These processes make it possible to institute the intellectual controls necessary for reliable, authentic, meaningful and accessible descriptive records to be carried forward through time. ${ }^{94}$

\footnotetext{
${ }^{91}$ VRA Core 4.0 Introduction, Library of Congress, 4. http://www.loc.gov/standards/vracore/VRA_Core4_Intro.pdf.

${ }^{92}$ For a complete history of EAD, see: Library of Congress, "Development of the Encoded Archival Description DTD." www.loc.gov/ead/eaddev.htmlhttp://www.loc.gov/ead/eaddev.html.

${ }^{93}$ The ISAD(G) Standards Document can be found here: http://www.icacds.org.uk/eng/ISAD(G).pdf. The ISAD(G) is currently implemented in the United States as Describing Archives: A Content Standard (DACS).

${ }^{94}$ Ibid, 7.
} 
EAD is made up of three high-level elements: (1) the header, which contains bibliographic and descriptive information about the archival finding aid; (2) front matter, which contains publication information about the finding aid; (3) archival description, which describes the content and context of the collection. EAD preserves the hierarchal nature of archival description and inheritance for collections, while also enabling access to digitized objects in collections when possible.

The Library of Congress began testing implementation of EAD finding aids in 1996. The pilot test included visual materials from the Print and Photographs Division, for the National Association for the Advancement of Colored People (NAACP) collection. After initial testing, the division concluded that EAD was suitable for providing access to photographic and pictorial materials; in fact, some items that were not in existing paper finding aids such as collection summaries were being added to EAD finding aids to provide more contextual information. ${ }^{95}$ EAD also enables access directly to material through the use of the $<$ daogrp $>$ element, which links to collections of digitized items.

At the same time, there was growing recognition by librarians and archivists that essential digital object attributes were not captured by descriptive metadata alone. The technical and administrative processes involved in the creation, ongoing maintenance, and long-term preservation of digital objects needed to be recorded. The Digital Library Federation spearheaded the Making of America II (MOA2) project in 1997 with five of its member institutions to propose standards for a national digital library. One goal of the project was to explore how to capture and record new types of metadata (termed "administrative" and "structural") deemed necessary for managing digital objects. The project resulted in the

\footnotetext{
${ }^{95}$ Mary A. Lacy and Anne Mitchell. "EAD Testing and Implementation at the Library of Congress," The American Archivist 60, no. 4 (1997): 431.
} 
formulation of the MOA2 Document Type Definition (DTD), which supported encoding descriptive, structural, and administrative metadata. Though the MOA2 DTD had several limitations and deficiencies, it helped lay the foundation for the development of an essential structural template for digital libraries - the Metadata Encoding \& Transmission Standard (METS) which was published in $2001 .{ }^{96}$ METS is a framework for managing complex digital objects in a digital library setting. It provides a hierarchal structure for packaging contextual, administrative, technical, and descriptive information about digital objects, including relationships with both external and internal content. In METS-packaged XML files, photographs and other visual materials can be represented through encoding in the XML document itself, through pointers to external sources, or through both. The current METS schema, now in its ninth iteration, is hosted at the Library of Congress.

In addition to structural, administrative, and descriptive metadata, preservation metadata emerged as a key concept in digital preservation activities. ${ }^{97}$ The development of the Preservation Metadata: Implementation Strategies (PREMIS) schema in 2005 provided a comprehensive structure for capturing and recording attributes deemed essential for long-term preservation and access. For visual materials, PREMIS metadata can be used to record critical events about the digitization process, which can help alleviate concerns over the provenance, authenticity and integrity of the original image. For example, suppose an institution digitizes a photograph to be ingested in an archival-quality tagged image file format (TIFF) format.

\footnotetext{
${ }^{96}$ For a complete history of the origins and implementation of METS, see: Jerome McDonough, "METS: Standardized Encoding for Digital Library Objects," International Journal on Digital Libraries 6, no.2 (2006): 148158, http://hdl.handle.net/2142/177.

${ }^{97}$ For a complete history of preservation metadata, see: OCLC/RLG Working Group on Preservation Metadata, "Preservation Metadata for Digital Objects: A Review of the State of the Art," January 31, 2001, https://www.oclc.org/content/dam/research/activities/pmwg/presmeta_wp.pdf.
} 
PREMIS can record extensive information about the original capture environment, including the digitization software, operating system, and digitizing "agent." It also can track and display information regarding an image's copyright. ${ }^{98}$

Though there are many metadata elements across schemas that can be used for managing digital objects in image collections, only one schema has been specifically designed for digital still images. Metadata for Images in an XML Schema (MIX) emerged in 2006 out of a collaborative effort between the Library of Congress and the National Information Standards Organization (NISO). MIX captures and records technical metadata for digital still images using four different types of metadata: digital object information, image information, image capture, and image assessment. The MIX schema contains specific details about the digitization process, such as the equipment used, resulting compression information, and image quality metrics. It also allows for the description of the analog source material type along with its original dimensions. Metadata extraction tools including the JSTOR/Harvard Object Validation Environment (JHOVE) and Digital Record Object Identification (DROID) can be used to output MIX technical metadata after reading source files.

The Metadata Object Description Schema (MODS) was developed by the Library of Congress' Network Development and MARC Standards Office in 2001 to provide a schema and guidelines for resource discovery. It contains a rich set of elements for describing both digital objects and digital collections. It allows for the use of any controlled vocabularies, including Library of Congress Subject Headings (LCSH) and TGM. The creation of a MODS record for a digitized photograph in an archival digital collection might contain information about the

\footnotetext{
${ }^{98}$ The PREMIS Data Dictionary specifies how to construct semantic units in PREMIS. It also provides examples about how to encode different object types using the schema, including digitized photographs. See: PREMIS Editorial Committee, PREMIS Data Dictionary for Preservation Metadata, version 2.0 (March 2008), http://www.loc.gov/standards/premis/v2/premis-dd-2-0.pdf.
} 
original photographer, subject descriptions based on the photographic content, the item's current repository location, and any digitization processes relating to the source object. ${ }^{99}$

Despite the existence of numerous metadata schemas, it is not clear how institutions are using them to describe their digital image collections. To date, there have been no surveys conducted which summarize and/or compare metadata schema usage across image collections. Additional research on metadata schema usage would complement efforts to standardize image description. Such investigations could also aid in uncovering potential metadata quality problems in image collections.

\subsubsection{The problem with visual materials: Implications for archival access}

Archivists have long acknowledged that visual materials such as photographs can provide rich, valuable, and unique cultural information. Yet collecting institutions have been notably slow to adapt and model descriptive standards specific to visual materials. Moreover, the archival literature has paid limited attention to the problem of visual material description and access. The few exceptions suggest that archivists should aim to develop visual literacy skills to improve description efforts. Though writing primarily about photograph appraisal, William Leary advises that archivists processing visual materials should be both "students of history" and "student[s] of the history of photography." 100 Elisabeth Kaplan and Jeffrey Mifflin advocate for archivists to familiarize themselves with visual communication methods and borrow aesthetic approaches from photography, film, and video to formalize literacies. ${ }^{101}$ In Photographs:

\footnotetext{
${ }^{99}$ The MODS User Guidelines provides examples for different material types, such as this entry for a digitized photograph which was an original 35MM slide digitized as a TIFF in 2003: http://www.loc.gov/standards/mods/v3/mods-userguide-examples.html\#digitized photograph.

${ }^{100}$ William H. Leary, The Archival Appraisal of Photographs: A RAMP Study with Guidelines (Paris: United Nations Educational, Scientific, and Cultural Organization, 1985): Section 2.5.2.

${ }^{101}$ Elisabeth Kaplan and Jeffrey Mifflin, “"Mind and Sight': Visual Literacy and the Archivist,” Archival Issues 21, no. 2 (1996): 96.
} 
Archival Care and Management, Helen Zinkham's chapter entitled "Reading and Research Photographs" provides an in-depth explanation of how archivists might describe photographic materials. She instructs archivists to study both photographs and negatives carefully, noting any written information on the materials themselves or on their containers. She describes potential reference sources such as pictorial histories, histories of photography, and photography dictionaries, directories, and databases that might help identify relevant details. ${ }^{102}$

There are inherent complexities in describing and providing access to visual materials for archives. Joan Schwartz argues that by "embracing a textual model of recorded information and by adopting a bibliographic model of image classification, archives continue to fixate on the factual content rather than the functional origins of visual images." ${ }^{103}$ For Schwartz, the continued use of descriptive standards and models developed for text-based materials ultimately complicates access to photographic materials. Jane Greenberg agrees that current descriptive standards place constraints on access to visual materials, particularly across domains. ${ }^{104}$ She analyzes metadata element usage from four common metadata schemas: (VRA Core, EAD, the Dublin Core, and Record Export for Art and Cultural Heritage (REACH)) and identifies four types of functional metadata classes: discovery, use, authentication, and administration. Rather than rely on domain-specific metadata schemas, Greenberg argues that one can better exploit access to images through the development of metadata schemas based on function rather than domain. She asks: "Why not permit the photographic archival image documenting the

\footnotetext{
${ }^{102}$ Helena Zinkham, "Reading and Researching Photographs," in Photographs: Archival Care and Management, ed. Mary Lynn Ritzenthaler and Diane Vogt-O’Connor (Chicago: The Society of American Archivists, 2006), 59-77.

${ }^{103}$ Joan M. Schwartz, "Coming to Terms with Photographs: Descriptive Standards, Linguistic 'Othering,' and the Margins of Archivy," Archivaria 54 (2002): 143.

${ }^{104}$ Jane Greenberg, "A Quantitative Categorical Analysis of Metadata Elements in Image-Applicable Metadata Schemas." Journal of the American Society for Information Science and Technology 52, no. 11 (2001): 917-924.
} 
construction of the Brooklyn Bridge to be accessible as an art image with aesthetic qualities and as a visual resource portraying state-of-the-art bridge engineering using wire cables during the latter half of the $19^{\text {th }}$ century?"105 In other words, metadata schema design should support functions across domains (such as discovery of images, regardless of disciplinary background) rather than focusing on designs to meet the needs of only one domain.

Given the relationship between archival description and access, the archival profession could benefit from a better understanding of the nature, purpose, and use of photographic materials in archives. What do users need from image collections in order to make sense and establish meaning? How do they experience visual information in the digital realm? Paul Conway and Ricardo Punzalan sum up the challenges: “Archivists, grappling with photography's idiosyncrasies as a medium, find it difficult to articulate photographic meaning, and have been uncomfortable about its place in the archives in relation to other holdings. This uneasiness has profoundly constrained our ability to handle photographs, in both practice and theory."106

As documents living in an archive, photographs are inextricably tied to their contemporaneous structures and cultural norms of the day. Such is the curse of visual materials which must be interpreted at some level to be described; how one interprets a photograph forty years from now may in fact differ quite dramatically from the ways in which it is interpreted and described today. In his important exposition, Tim Schlack uses archival literature from the 1970s forward to show changing conceptions of the archival photograph, moving from image as

\footnotetext{
105 Ibid, 921.

106 Paul Conway and Ricardo Punzalan, "Fields of Vision: Toward a New Theory of Visual Literacy for Digitized Archival Photographs," Archivaria 71 (2011): 68.
} 
evidential historical fact to the more recent status of image as representation. ${ }^{107}$ The archivists' burden is that in each of these contested spaces, a photograph's description must endure. As Schlack writes, "Working with photographs then is a process of substantiating the most valid narrative that a photograph collection can evoke and transcribing it into the academic discourse of our time." ${ }^{108}$ For archivists, the great obstacle remains how to effectively situate the photograph, whose meaning and interpretation is only ever temporally fixed, in archival spaces that exist in perpetuity.

\subsection{LIS Approaches to Studying Image Use}

There is a noticeable absence in the LIS literature of qualitative examinations of information use in image collections. The empirical work on image collection use tends to focus on the following themes: identifying information needs to improve image retrieval, analysis of user queries submitted to retrieval systems, and examination of transaction logs or citations to quantify usage statistics. Few studies examine aspects of the user experience interacting with images. What are the qualitatively different ways in which users interact with visual information in the digital realm? In this section, I will briefly review the LIS literature on use of image collections. How has use in image collections been studied? First, I will review studies which use query analysis as the basis for understanding information needs in image retrieval systems. Next I will explore task-based approaches to image collection use. Finally, I will review the literature that examines experiential aspects of use in image collections.

\footnotetext{
${ }^{107}$ Tim Schlack, "Framing Photographs, Denying Archives: The Difficulty of Focusing on Archival Photographs," Archival Science 8, no. 2 (2008): 85-101.

${ }^{108}$ Ibid, 96.
} 


\subsubsection{Query analysis}

Linda Armitage and Peter G.B. Enser collected and analyzed approximately eighteenhundred queries for image-related materials submitted to seven libraries. ${ }^{109}$ Queries were then categorized into facets derived using the Panofsky-Shatford image analysis matrix. The authors conclude that image classification schemas can be adopted and implemented by libraries to support more effective visual information retrieval. However, they point out that particular attributes may not always be represented at the correct level of specificity to satisfy user needs. For example, they describe the difficulties in accurately classifying queries which describe geographic locations that are non-specific (e.g., "London scenes") or singular topics that may include many types of representational formats (e.g., paintings, photographs, or films featuring a famous person).

Karen Collins also studied user queries in two photographic archives to assess whether information systems for visual materials (including current indexing techniques) were meeting the information needs of users. ${ }^{110}$ Her results reveal that subject terms are used more often than other classes of attributes and that patrons infrequently search for images based on visual requirements (e.g., color versus black and white). Another significant finding is that a very small number of patrons (four percent, or seven out of 187 participants) queried images by image creator. In fact, despite the perceived importance and stronghold of provenance as an archival access point, all of the images were sought individually rather than in the context of a collection. Noting this discrepancy, Collins writes: "While maintaining the context in which images were

\footnotetext{
${ }^{109}$ Linda H. Armitage and Peter G.B. Enser, “Analysis of User Need in Image Archives,” Journal of Information Science 23, no. 4 (1997): 287-299.

${ }^{110}$ Karen Collins, "Providing Subject Access to Images: A Study of User Queries," The American Archivist 61, no. 1 (1998): 36-55.
} 
created or used is necessary to preserve their evidential value, it is clear that few patrons are presently using images as primary source documents. A study of how patrons use images, and the implications for archives, would be interesting and useful."111

Chung and Yoon used the Yahoo Answers database as their corpus to investigate imagebased queries. ${ }^{112}$ They examined 474 image-seeking related questions submitted to the site, such as the following: "Where can I find wallpaper of FIFA Club World Cup Finals Barcelona picture?" The authors attempted to classify each question into one of the following seven types of use: illustration, information processing, information dissemination, learning, generation of ideas, aesthetic value, and emotive and persuasive use. ${ }^{113} 192$ queries were each sorted into an image use classification; the most prominent use was illustration $(40 \%) .{ }^{114}$ Contextual information was found to be very important in deciding whether to use an image. For example, textual information such as image captions and associated text should be indexed when possible. User interfaces should also be expanded to accommodate richer descriptions of images. Chung and Yoon explain:

As the findings of this study suggest that there are needs for both concept-based and content-based indexing approaches, therefore image retrieval systems, especially image search engines on the web, need to embrace two aspects of image needs. Practically, in order to imply both concept and content-based approaches into search engines, both indexing and user interface issues might be resolved concurrently. ${ }^{115}$

\footnotetext{
${ }^{111}$ Ibid, 52

112 EunKyung Chung and JungWon Yoon, "Image Needs in the Context of Image Use: An Exploratory Study," Journal of Information Science 37, no. 2 (2011): 163-177.

113 These seven classes were adapted from the following: L.R. Conniss, A.J. Ashford and M.E. Graham, Information Seeking Behavior in Image Retrieval: Visor I Final Report: Library and Information Commission Research Report 95 (Newcastle Upon Tyne: Institute for Image Data Research, 2000).

${ }^{114}$ EunKyung Chung and JungWon Yoon, “Image Needs in the Context of Image Use,” 169.

115 Ibid, 175.
} 
The researchers recommend that future studies employ a holistic perspective to investigate why and how users seek images.

Using the Library of Congress' American Memory Collection as their corpus, Youngok Choi and Edie Rasmussen investigated both the search queries submitted by graduate students and faculty and broad categorizations of queries in participants' stated information needs. ${ }^{116}$ Thirty-eight search requests were analyzed and categorized according to the specificity of their description. ${ }^{117}$ The most frequently assigned query type (twenty-three, or $60 \%$ ) was general or nameable needs expressed in key words. ${ }^{118}$ Participants were also asked to rate the relevance of textual attributes describing images. Date, title, and subject descriptors were found to be the most important factors for participants.

\subsubsection{Task-based analysis}

Raya Fidel sought to investigate the extent to which tasks influence image retrieval. ${ }^{119}$ Using the twelve classes of image attributes identified by Jorgensen, she analyzed and classified 100 queries submitted to a stock photography agency. ${ }^{120}$ Fidel notes semantic differences between user queries that were motivated by informational purposes (such as an icon for a

\footnotetext{
${ }^{116}$ Youngok Choi and Edie M. Rasmussen, "Searching for Images: The Analysis of Users' Queries for Image Retrieval in American History," Journal of the American Society for Information Science and Technology 54, no. 6 (2003): 498-511.

117 The authors adopted their categories from Susan Batley’s 1988 work. They used four information need categories: (1) specific needs naming person, event, or activity; (2) general or namable needs expressed in key words; (3) abstract needs involving abstract concepts; (4) subjective needs dependent on interpretation. See: Susan Batley, "Visual Information Retrieval: Browsing Strategies in Pictorial Databases," in Online Information 88. Proceedings of the Twelfth International Online Information Meeting (Oxford, Learned Information LTD., 1988), 373-381.

${ }^{118}$ Choi and Rasmussen, "Searching for Images," 504.

${ }^{119}$ Raya Fidel, "The Image Retrieval Task: Implications for the Design and Evaluation of Image Databases,” New Review of Hypermedia and Multimedia 3, no. 1 (1997): 181-199.

${ }^{120}$ See Figure 1 for Jorgensen's list of attributes.
} 
handicapped entrance) versus images sought as representations (such as a colorful picture). ${ }^{\mathbf{1 2 1}}$ These conceptual differences in query construction suggested to Fidel that the nature of the retrieval task might impact search behavior. To account for possible differences in behavior, Fidel developed two polar construct categories to perform further analysis: Data Poles and Object Poles. Data Poles could be used to describe retrieval tasks in which relevance criteria are most likely known ahead of time. For example, a user searching for a picture of a Chevy Malibu will attempt to retrieve a specific image that meets his or her already-stated criteria. The user will be able to confirm the relevance of an image based on the specifics of his or her information need (which explicitly states the "data" needed to find relevant images). At the other end of the spectrum, Object Poles will likely describe retrieval tasks in which the user still needs to evaluate post-retrieval whether that search result has met his/her information need. Fidel describes Object Pole retrieval tasks as ones in which "users are more likely to have difficulties expressing relevance criteria ahead of time, but more likely to recognize these criteria when they 'see' them."122 Though Fidel provides no further empirical explorations into the matter, her research highlights at a theoretical level the conceptual differences in image search behavior (and possible subsequent use) when users have specific information needs in mind for their retrieval tasks. In her discussion, she suggests further research should be undertaken to see how traditional textbased retrieval measures like precision and recall could be adapted for the complexities of visual information search tasks.

\footnotetext{
${ }^{121}$ These two examples were used by Fidel to describe the difference between what she terms "data objects" and "information objects."

${ }^{122}$ Ibid, 191.
} 
Lori McCay-Peets and Elaine Toms also focused on task-based work contexts, interviewing thirty journalists and historians to better understand motivations in using images. ${ }^{123}$ Building on Fidel's previous work, McCay-Peets and Toms were interested in classifying whether participants used images as "illustration" or "information." Participants were asked to recall incidents when they needed to use an image for work and then asked to identify at which point in their work process they used the image. They were shown a list of twelve attributes related to image description, which were also categorized as either "descriptive" or "conceptual" types of attributes. Participants were then asked to identify the attributes which would satisfy potential needs for image use. The following work task stages were examined: Idea generation, Preparation, Elaboration, Analysis/Writing, and Dissemination. The majority of participants $(80 \%)$, reported image use that researchers categorized as "illustration." ${ }^{124}$ On average, participants in both the "illustration" and "information" categories identified three key attributes for their image needs, with both groups ranking "people/animal/object" as the most important attribute. One of the most interesting findings emerging from this study is a clear demonstration that image needs change depending on participants' workflow stages. The researchers give the following example:

One participant noted that while he needed to obtain an image for illustrative purposes early in his work task process (Preparation stage) for an article he was writing, it was because he wanted to make sure he had enough time to acquire it before publication. The image was not really used until the Dissemination stage when the image was made ready for publication. ${ }^{125}$

\footnotetext{
${ }^{123}$ Lori Mccay-Peet and Elaine Toms, "Image Use within the Work Task Model: Images as Information and Illustration," Journal of the American Society for Information Science 60, no. 12 (2009): 2416-2429.

${ }^{124}$ Though the researchers define the terms "illustration" and "information," they do not describe how they determined and classified image use as one or the other.

${ }^{125}$ Ibid, 2425.
} 
McCay-Peets and Toms recommend that image needs be further delineated to effectively distinguish motivations between image acquisition (physically acquiring the item) and image use (purposefully using the item). They also call for more user studies examining the relationship between image selection and work tasks, so that information retrieval models can better accommodate user information needs.

Markkula and Sormunen examined the practices of journalists working in a digital photo newspaper archive in Finland. ${ }^{126}$ They were able to identify types of relevance criteria employed by journalists in their selection of images. Topicality was considered an initial indicator of relevance - image captions were used to effectively authenticate whether images were appropriately topical illustrations. Other criteria used to assess images included contextual and aesthetic attributes. The technical quality of the photograph was always mentioned by journalists as a factor in determining relevancy. It is important to note that the researchers concluded that examining user queries from image searches revealed very little about user behavior to investigators; instead, observation sessions from the newsroom provided a more illuminating context for image needs and uses. ${ }^{127}$

\subsubsection{Experiential analysis}

A pilot study conducted by Wendy Duff, Emily Monks-Leeson, and Alan Galey is one of the few empirical examples from the archival literature which seeks to investigate the experiences of individuals interacting with archival documents. ${ }^{128}$ Importantly, the authors focus

\footnotetext{
${ }^{126}$ Marjo Markkula and Eero Sormumen, "Searching for Photos - Journalists' Practices in Pictorial IR," Challenge of Image Retrieval Workshop (University of Northumbria at Newcastle, Newcastle upon Tyne, 1998): 1-13.

${ }^{127}$ Ibid, 4

${ }^{128}$ Wendy Duff, Emily Monks-Leeson, and Alan Galey, "Contexts Built and Found: A Pilot Study on the Process of Archival Meaning-making," Archival Science 12, no. 1 (2012): 69-92.
} 
on the process of how users interpret archival materials, rather than how they find or search in the archives. The authors observed three distinct stages of behavior taking place: (1) making guesses and hunches about archival record content; (2) conception of materials using a predetermined framework; and (3) identification of relevant concepts among archival records. ${ }^{129}$ The authors also found that domain knowledge had a critical impact on the process of meaningmaking in the archives. Users browsed archival records more purposefully and could make connections among records when they were able to draw from existing conceptual frameworks.

In 2010, Kathleen Fear examined the perceived usefulness of the Dublin Core metadata elements for users working with digital image collections. ${ }^{130}$ Her sample population consisted of seventy-eight individuals, largely undergraduate students, who each participated in three separate evaluative activities as part of the research study. Fifty students filled out a questionnaire, including two evaluation tasks. Eighteen students participated in two different focus group sections, and ten students completed a search/usability session.

One of Fear's most interesting findings is that users considered the primary source of information to be the image itself, rather than any accompanying metadata or related contextual details. Image contents can be quickly and easily evaluated to determine whether to continue searching. The second most frequently mentioned factor considered important to participants is the size of an image (both in terms of image resolution and dimensions). Participants also want assurance that images come from reliable environments; nineteen people indicated that information about where an image originated and/or who posted it are relevant factors.

\footnotetext{
${ }^{129}$ Ibid, 80.

${ }^{130}$ Kathleen Fear, "User Understanding of Metadata in Digital Image Collections: Or, What Exactly Do You Mean by 'Coverage'?” The American Archivist 73, no.2 (2010): 26-60.
} 
Although the stated purpose of her study was to examine search behavior, Fear's research also offers rich, nuanced descriptions of user interactions with images in the context of an online collection. In the usability session, for example, participants were asked to perform two search tasks and then to comment on any factors that influenced their decision to select (or not select) an image for use. Fear describes wanting to ascertain the following about the user experience:

Did they understand all the elements on the page and their content? Which elements did they use and which did they ignore? What information that was not included would they like to have seen, and, conversely, what provided information did they think could have been excluded? ${ }^{131}$

Such accounts provide valuable insight into the factors that may impact user experience and satisfaction with finding and using digital resources. They also highlight the complexities of interacting with visual information. In fact, participants frequently mention visual components (e.g., the presentation and display of information, zoom capacities for images, mouse-overs) when describing desired functionalities. ${ }^{132}$

Acknowledging the dearth of empirical investigations into visual information use, Joan Beaudoin investigated four types of user groups who use images in their professional work: archaeologists, architects, art historians, and artists. ${ }^{133}$ She conducted surveys and semistructured interviews with four recruited participants from each group. Each group was asked questions relating to: (1) how they used images in the course of work processes and (2) why they used images as part of the products they created. Beaudoin then analyzed and coded participant responses to thematically compare functional reasons given for image use.

\footnotetext{
${ }^{131}$ Ibid, 39.

132 Ibid, 49.

${ }^{133}$ Beaudoin, “A Framework of Image Use.” One complicating factor to note is the author's broad use of the term "image" to mean anything from a digital mock-up on a screen to a picture of a painting.
} 
Knowledge development as a motivating factor for image use was the single category shared across all four user groups. Images also served as conceptual models for two groups of users, architects and artists, with continuous refinement throughout the creative process. Architect 6, for example, describes the process of solidifying design concepts using visual methods:

It will start out with one of the partners doing a sketch... a hand-drawing... really quick. 'Build this.' And we translate that into a 3-D model and then print it out and show it to him. 'Is this it?' 'No, no, no, no,' and then he will sketch back over the image we have just printed out. It is very much a back and forth, iterative process. ${ }^{134}$

Architects and artists also mentioned their use of images as sources of inspiration, generally in the beginning stages of their creative process; this is not surprising given that both user groups created visual materials as part of their work. Beaudoin credits architects as having the most diverse image needs among the user groups represented. On the other side of the spectrum, archaeologists and art historians tend to use images in their output - for presentations and teaching materials. Only one art historian explicitly addressed using images for research. Beaudoin points out that participants in academia were the least able to offer compelling evidence for how they use images to increase knowledge.

Many of the participants mention the "informational" components in images they use. Given the preponderance of image use motivated by knowledge development among research participants, this may not be surprising. For example, half of the artists mention using images explicitly for the information they contained. ${ }^{135}$ Still, the idea that images can be read and

\footnotetext{
${ }^{134}$ Ibid, 24-25.

${ }^{135}$ Ibid, 19.
} 
understood as visual information suggests an important conceptual shift in the paradigm of image use and information.

Paul Conway and Ricardo Punzalan explore the crucial role of meaning making in interactions with digitized visual materials among archive users. ${ }^{136}$ The authors introduce "Fields of Vision," a first attempt in the archival literature to model how users interact with digitized images in the context of their use. Conway and Punzalan conducted in-depth interviews and surveys with seven participants who could be summarized as expert, non-academic users of photographic materials. Using grounded theory analysis, the researchers identify three different modes of inquiry in which participants use digitized images. In the Discovering mode, participants "obtain visual information from individual digitized images that has not been seen or noted previously." 137 For example, Participant 7 had a baseball history project in which he used photographs to identify unknown major league baseball players. The second mode identified by Conway and Punzalan is Storytelling, wherein participants assemble images to create narratives. An example is Participant 3, who selected images for use in research based on their ability to evoke compelling storylines. The final mode is Landscaping, in which the participant draws on external factors to contextualize the photograph in use. In this mode of inquiry, "digitized photographs may serve primarily as mnemonic devices, as illustrations for a primarily textual narrative, or as a lens on events and activities that took place beyond the view of the camera itself." 138 Participant 2 used the Landscaping mode, for example, to investigate the socio-

\footnotetext{
${ }^{136}$ Paul Conway and Ricardo Punzalan, "Fields of Vision: Toward a New Theory of Visual Literacy for Digitized Archival Photographs," Archivaria 71 (2011): 63-97.

${ }^{137}$ Ibid, 77.

${ }^{138}$ Ibid, 85 .
} 
political context in which Russian ethnographic photo albums are created. In describing her selection and use of one particular photograph of a horse bazaar, she explains:

I'm selecting images that convey culturally particular aspects that the Russians seem to be honing in on; horse bazaars are ancient in Central Asia. But this one is also political because the Russians were very interested in keeping up their cavalry. So they might be conveying not just an ancient industrial commercial practice of central Asians but also that they're deeply invested in controlling the land. ${ }^{139}$

While modes may intersect during the course of activities (e.g., a participant can use both Storytelling and Discovering mode), each mode has distinctive qualities that define the interaction. Four participants used two different modes, and the remaining three used one. It is important to note that Conway and Punzalan characterize participant modes based on narratives relayed by participants rather than through direct observations of interactions.

\subsection{Historians and the Use of Photographs as Evidence}

Despite the enormous growth in access to primary source materials, the use of visual materials in the discipline of history remains in its infancy. In a 2010 essay, Sarah Farmer argues that, "Historians of the western experience in the 19 th and $20^{\text {th }}$-centuries still overwhelmingly depend on text-based archives, although interdisciplinary research projects, conferences, and publications in the last ten years indicate a 'visual turn' in the scholarship."140 More recently, in a review of image use in historical journals from 2000-2009, Harris and Hepburn note that "use of images has remained at a relatively consistent level year to year."141 Why are historians remiss to use photographs as primary historical sources?

\footnotetext{
139 Ibid, 90.

${ }^{140}$ Sarah Farmer, "Going Visual: Holocaust Representation and Method," American Historical Review 115 , no. 1 (2010): 116. It is important to note that Farmer's characterization of a "visual turn" is regarding academic scholarship overall rather than specifically the discipline of history. In her examples, she cites cultural studies/media studies texts to indicate the visual turn.

${ }^{141}$ Valerie Harris and Peter Hepburn, "Trends in Image Use by Historians and the Implications for Librarians and Archivists," College and Research Libraries 74, no. 3 (2013): 276.
} 
Drawing on literature primarily from the historical discipline, this section will explore issues and conceptual challenges associated with historians' scholarly use of photographs as visual evidence. ${ }^{142}$ The first section will offer an overview on how concepts of evidence emerged in modern historiography. How is evidence constituted? What role does it play in establishing historical narrative? What techniques and methods do historians use to evaluate primary source documents as evidence? How has postmodernism and the so-called "new history" influenced conceptions of evidence? The second section will investigate how historians approach the use of photographs as visual evidence. What are the conceptual challenges and obstacles in using photographs as the basis for historical understanding? What affordances do photographs and visual materials overall offer to historians? In an attempt to demonstrate methods and present historical models, case studies on how historians have used photographs as visual evidence will be explored.

\subsubsection{Concepts of evidence in modern historiography}

The use of source materials as evidence forms the foundation of modern historiography. Methodological shifts in the first half of the $19^{\text {th }}$-century, in part due to the writings of the German historians Leopold Von Ranke and Friedrich Schlozer, resulted in historical practices that emphasized the formulation of empirical evidence. ${ }^{143}$ The processes by which source materials became evidence, enabling historians to make assertions and support historical arguments, thereby became of increasing interest to historians. The need to establish criteria to

\footnotetext{
${ }^{142}$ Rather than present a review of all the literature in the discipline of history, I limited my literature search to academic journals in an attempt to: (1) gain a sense of the state of the art and (2) investigate historians' conceptions about their use of visual materials as primary sources.

${ }^{143}$ Kasper Eskildsen, “Leopold Ranke's Archival Turn: Location and Evidence in Modern Historiography,” Modern Intellectual History 5, no. 3 (2008): 425-453. doi:10.1017/S1479244308001753.
} 
validate the accuracy, reliability, and authenticity of source materials was seen as paramount to historical practices.

In an early publication devoted entirely to the subject of historical evidence, the Reverend HB George describes the process by which evidence is formulated:

It only becomes evidence if, and so far as, it is used as the basis for an inference. The inference may be of any kind, from the simplest and most direct, to something remote: it may be cogent or merely suggesting a presumption or it may be entirely baseless. Whatever the value or the nature of the inference, the statement which gives occasion for it is, to the mind of the hearer, evidence. ${ }^{144}$

For George, the act of making inferences from statements is the establishment of evidence; importantly, such acts do not translate evidence directly into truth. Rather, the historian's role is to sift through source materials and make cumulative speculations. ${ }^{145}$ Similarly, William Henry Simcox describes the construction of evidence as a "double process" emerging from "a weighing and a sifting." 146 This recognition in turn sets up a framework by which source materials can be understood and evaluated as the basis for historical argument.

Historiographical literature in the $19^{\text {th }}$-century also emphasized that historians should be able to produce the material basis for their claims. In Sir George Cornewall Lewis' 1855 treatise on the credibility of the history of Rome, he argues, "It is not enough for a historian to claim the possession of a retrospective second-sight, which is denied to the rest of the world; of a mysterious doctrine revealed only to the uninitiated. Unless he can prove as well as guess; unless he can produce evidence of the fact, after he has intuitively perceived its existence, his historical

\footnotetext{
${ }^{144}$ H.B. George, Historical Evidence (Oxford: Clarendon Press, 1989), 14.

${ }^{145}$ Ibid, 28.

${ }^{146}$ William Henry Simcox, Principles of Historical Evidence Considered in Their Bearing on the History of Remote Times (Oxford: T. \& G. Shrimpton, 1868), 2-3.
} 
system cannot be received." ${ }^{147}$ Depending on one's perspective, source materials serve doubly in this capacity: as evidential proof for historical claims and as the building blocks from which historical arguments are crafted. The perception emerged that historical interpretation could only be as accurate as source materials. As Johnson explains, a historian "may hit upon a true statement of the facts, but he can rise no higher than the source from which he draws his information." $" 148$

Historians should gather as much information from varying source materials as possible. They must consider the specific conditions in which a historical source was produced, taking care to avoid assuming its reliability. ${ }^{149}$ Johnson outlines the extensive steps he would take in gathering disparate source materials were he tasked with writing about a particular historical event (in this case, the assassination of Major General Lee in Egypt). For instance, he mentions making sure to take eyewitness testimony from both "natives and foreigners," as well as members of the government. ${ }^{150} \mathrm{He}$ also mentions that he would visit the scene of the murder in an attempt to map out the positions of the parties involved. Admittedly, such actions are not always possible; nevertheless, Johnson's recommended practices gives insight into the importance that some $19^{\text {th }}$-century historians placed on assembling varying source materials.

\footnotetext{
${ }^{147}$ George Cornewall Lewis, An Inquiry Into the Credibility of the Early Roman History (London: J.W. Parker and Son, 1855), 15.

${ }^{148}$ Allen Johnson, The Historian and Historical Evidence (New York: C. Scribner's Sons, 1926), 2.

${ }^{149}$ Martha C. Howell and Walter Prevenier, From Reliable Sources: An Introduction to Historical Methods (Ithaca, N.Y: Cornell University Press, 2001), 19.

${ }^{150}$ Johnson, The Historian and Historical Evidence, 3.
} 


\section{Types of sources}

Historians describe the type of sources used in historical scholarship in a variety of ways. Source materials can be lumped into broad categorizations; for example, George describes sources as either direct historical statements, such as written documents, or indirect sources, contextual information which possess "none of the characteristics of documentary evidence."151 Johnson is equally vague broad in his depiction, differentiating between "records" and "remains." 152 Both kinds of sources are forms of testimony based on direct and indirect observation. It is the job of the historian, Johnson argues, to collect and collate sources and to use them appropriately in historical interpretation.

Other historians are more detailed in their source typologies. Simcox describes four forms of direct evidence: oral tradition, which can be evaluated in tandem with written records; monument inscriptions, which illustrate or authenticate stories from other sources; fragmentary literature, such as the Old Testament scriptures; and historical compilations, which combine many different sources. ${ }^{153}$ While Howell and Prevenier only distinguish two categories of sources, written and non-written, they describe in detail the types of materials that constitute those categories. Moreover, they recognize the emergence of new types of source materials, and thus new methodologies, due to widespread technological changes. ${ }^{154}$

\footnotetext{
${ }^{151}$ George, Historical Evidence, 28.

${ }^{152}$ Johnson, The Historian and Historical Evidence, 4.

${ }^{153}$ Simcox, Principles of Historical Evidence, 17-19.

${ }^{154}$ Howell and Prevenier, From Reliable Sources, 30.
} 


\section{Establishing trustworthy sources}

The types of source materials historians employ undoubtedly impact their historical interpretation. Some materials are recognized as more trustworthy while others are considered less reputable. Recorded observations of an event, for example, often do not hold the same weight for many historians as eyewitness testimony does. The literature has addressed these nuances in two ways: (1) to outline acceptable historical practices and (2) to discuss methods and techniques for assessing the credibility of sources.

$19^{\text {th }}$-century historiography takes an abstract approach to the nature of trustworthy historical practice. In some cases, this comes in the form of outlining specific character traits for historians. In his 1830's manuscript “On the Character of Historical Science," Leopald Von Ranke describes the historian as needing to show "a pure love of truth" and a "universal interest" in all aspects of society. Historians must be impartial, observational, logical, and able to look at the "conception of the totality" surrounding events or people. ${ }^{155}$ In other writings, particular documents are contrasted to show how they offer more credible approaches to historical inquiry. Such demonstrations do not translate into instructive historical practices; for example, Forsyth argues that the New Testament is a more credible account than the legends of the Saints based on the number of eyewitnesses and the fact that it explains "the origin of a religion that has lasted eighteen centuries." ${ }^{156}$ Nevertheless, they do offer valuable insight into conceptions of authentic historical practices in the $19^{\text {th }}$ century.

\footnotetext{
${ }^{155}$ Leopold von Ranke, "On the Character of Historical Science," in The Theory and Practice of History, ed. Georg G. Iggers, Konrad von Moltke, Wilhelm Humboldt and Leopold von Ranke (Indianapolis: Bobbs-Merrill, 1973), 1415.

${ }^{156}$ William Forsyth, The Rules of Evidence As Applicable to the Credibility of History (London: Robert Hardwicke, 1874), 9.
} 
The methods through which historians validate the credibility of their evidence range from common sense approaches to detailed technical analysis. In the case of eyewitness testimony, George argues that historians must evaluate the witnesses' ability to know the truth, capacity for accuracy, and trustworthiness. ${ }^{157}$ Simcox recommends looking at other statements made by the witness to help establish credibility. ${ }^{158}$ Johnson describes the importance of evaluating the external characteristics of source materials: dating materials, provenance, and authorship. He explains, "The appraisal of historical material involves, first of all, determination of the time when it was fabricated, written, or printed, and of the place where it originated; then, the more difficult determination of its authorship." 159

Other methodological approaches call for the comparison of differing accounts of the same event. Tasked with examining how Rome's early history was written, Lewis explains his approach:

We shall first endeavor to ascertain the general character of the sources from which the extant narrative of the early centuries of Rome have been derived, and to discover how it came to assume the form in which it has been delivered to modern times. When this part of our task has been accomplished, it will be necessary to proceed one step further, and to examine the extant narrative, in order to try how far its internal character and composition, and its external attestation, agree. It has been truly remarked that a critical inquiry into the credibility of the early Roman history can scarcely be separated from a positive exposition of the facts out of which that history is formed. ${ }^{160}$

By looking at conflicting versions and applying tests of credibility, historians should conceivably be able to assess which perspective is the most valid. Interestingly, these approaches all

\footnotetext{
${ }^{157}$ George, Historical Evidence, 31.

${ }^{158}$ Simcox, Principles of Historical Evidence, 39.

${ }^{159}$ Johnson, The Historian and Historical Evidence, 51.

${ }^{160}$ Lewis, An Inquiry into the Credibility of the Early Roman History, 16.
} 
presuppose the existence of an objective truth from which historians can measure or judge statements based on their accuracy.

\section{Evidence and the new history}

During the $20^{\text {th }}$ century, historiographical perspectives underwent a radical shift. The emergence of a paradigmatic approach known as "the new history" could be seen as a "direct reaction" to the Rankean approaches which dominated $19^{\text {th }}$-century historiographical writings. ${ }^{161}$ The positivist bent of the Rankean paradigm was predicated on the notion that historical truth could be obtained from carefully evaluated sources and used to construct narrative; the new history called into question the formulation and usefulness of such approaches. It favored the expansion of historical methodologies, types of source materials, and objects of study. Perhaps most importantly, there was greater acknowledgement that historians themselves were instrumental in shaping historical understanding. In his 1933 presidential address to the American Historical Association, Charles Beard describes the role of the historian: "Facts, multitudinous and beyond calculation, are known, but they do not select themselves or force themselves automatically into any fixed scheme of arrangement in the mind of the historian. They are selected and ordered by him as he thinks."162

Bolstered by the influence of modernism and structuralism, historical scholarship encountered "a crisis in representation." 163 Once positioned rather innocuously, the historical document was now instead seen as a mediated object of representation. There were also significant shifts in the practices used to craft historical arguments. According to Lawrence

\footnotetext{
${ }^{161}$ Peter Burke, New Perspectives on Historical Writing (University Park: Pennsylvania State University Press, 1992), 2-3.

${ }^{162}$ Charles A. Beard, "Written History as an Act of Faith,” The American Historical Review 39, no. 2 (1934): 220.

${ }^{163}$ Robert Berkhofer, Beyond the Great Story: History as Text and Discourse (Cambridge: Harvard University Press, 1997), 3.
} 
Stone, the new history favored the use of analytic frameworks rather than the narrative tendencies used previously. ${ }^{164}$ Such changes in perspective and practice had profound implications for notions of historical understanding and conceptions of evidence in historical argument.

In his foundational text Narration and Knowledge, Arthur Danto argues that we cannot disengage historical understanding from its temporal dimensions. It is only through the distancing enabled by the experience of seeing history-as-actuality that one can in turn adequately perceive history-as-record. In other words, one can only understand the past as such by experiencing it in the present. He explains:

This is certainly a difficult notion to analyze, partly at least because - if I may protract the metaphor which does not quite permit one to say what one wishes - it is only by 'looking through the curtain and beyond' that we can see the fabric. Less metaphorically, just to apprehend something as evidence is already to have gone beyond the state of merely making statements about $i t$ : to count something as evidence is already to be making a statement about something else, namely, that for which it is taken as evidence. And taking $E$ as evidence for $O$ is to see $E$ differently from the way we would if we had no notion at all about $O$. Thus, just to see something as evidence is already to be 'looking through the fabric and beyond. ${ }^{165}$

While Danto was a philosopher of history rather than a historian, his writings reflect the changing perspectives on historical thinking and understanding that emerged during the $20^{\text {th }}$ century.

Robert Berkhofer takes Danto's points one step further. In Beyond the Great Story, Berkhofer argues that common historical practice (what he terms "normal history") relies on the ideological use of information which has been already historicized as fact. In the course of accepted methods for normal historical practice, historians derive facts from sources that are

\footnotetext{
${ }^{164}$ Lawrence Stone, The Past and the Present Revisited (London: Routledge \& Kegan Paul, 1987), 21.

${ }^{165}$ Arthur Danto, Narration and Knowledge (New York: Columbia University Press, 2007), 89.
} 
considered capable of representing the past. "To consider historical sources as evidence of the past, historians predicate that they remain from past real events and behaviors and bear such a relationship to those past realities that the historian can reconstruct those past events and behaviors from them." ${ }^{166}$ In the normal history paradigm, the historian uses sources as evidence to construct narratives for what Berkhofer calls "The Great Story" of history.

A key component in understanding the "normal history" paradigm is how evidence figures into the historical process. In the normal history method, evidence is isolated, evaluated as fact, and then synthesized. This stands in contrast to a meta-history or rhetorical approach which positions the text as the "vehicle for representing the past as history." 167 The latter approach illuminates how the "referential facts" that historians use to craft historical arguments are themselves already historicized. In other words, as Keith Jenkins explains in his reading of Berkhofer's argument, the process of facts being used as historical constructs means they have already become representations rather than (mere) references. He writes that "the effect normal historians try to achieve in their representations is the fusion of the structures of interpretation and factuality to try and prove that the structure of interpretation is the structure of factuality, instead of showing how the representation is structured 'to look like total factuality."'168 Thus, normal historical practice, which prescribes the synthesis of facts through interpretation, is always already incapable of treating those facts as anything but objectively truthful.

Berkhofer argues that by demystifying the practices of normal history, we can expose how historical discourse functions as a mode of historical representation. By separating out the

\footnotetext{
${ }^{166}$ Berkhofer, Beyond the Great Story, 29-30.

${ }^{167}$ Ibid, 70.

${ }^{168}$ Keith Jenkins, The Postmodern History Reader (London: Routledge, 1997), 19.
} 
analysis of text, discourse, and story, historians can begin to decode the ideological structures that present and legitimize the practices of normal history. Such practices can then be superseded by new forms of historical representations, which can use contextualist approaches to move beyond the Great Story.

Despite the recognition that historians employ ideologies in their interpretations, there are few empirical works examining how historians construct evidence. One exception is Samuel Wineburg's dissertation work on historical problem-solving and understanding. ${ }^{169}$ Wineburg attempts to model the cognitive processes that underlie acts of historical reading and interpretation. He identifies three kinds of heuristics used by historians that characterize historical understanding. First, historians use corroboration - or the act of comparing documents with one another. As an example of a measure of corroboration, Wineburg refers to the number of times historians looked back at previous documents to verify or double-check facts. A second heuristic is sourcing-or the act of looking at the source or attribution of the document. A third heuristic is contextualization - or "the act of situating a document in a concrete temporal and spatial context." 170 According to Wineburg's findings, historians employ all three of these heuristics in the act of constructing historical arguments.

\subsubsection{Historians and photographic use}

Modern historiographical approaches recognize the importance of evaluating primary source materials as historical evidence. While the source-criticism techniques popularized by Ranke in the nineteenth century helped lay the groundwork for methodological approaches

\footnotetext{
${ }^{169}$ Samuel Wineburg, "Historical Problem-Solving: A Study of the Cognitive Processes Used in the Evaluation of Documentary Evidence" (PhD diss., Stanford University, 1990).

${ }^{170}$ Samuel Wineburg, "Historical Problem Solving: A Study of the Cognitive Processes Used in the Evaluation of Documentary and Pictorial Evidence.” Journal of Educational Psychology 83, no. 1 (1991): 77.
} 
which incorporated critical examination of primary sources as texts, the so-called "new history" or "cultural turn" of the 1960s held important ramifications for existing conceptions of both historical argument and evidence. ${ }^{171}$ Postmodernists' and poststructuralists' critical examination of discourse and the legitimizing social structures of the historical canon expanded the research agenda. In the discipline of history, the influence of postmodernism helped orient historians away from the study of significant figures and towards the "history of ordinary people and everyday life." ${ }^{172}$ These shifts in focus toward new kinds of subjects and subject matter encouraged the use of different types of source materials as historical evidence.

In particular, photographic archives presented a host of opportunities for historians studying class, gender, and race. Insights provided by photographic evidence could allow historians to fill in under-developed, underrepresented or misrepresented cultural histories. In his introduction to a special issue in the Journal of Pacific History on photography and history, Quanchi explains that "visual histories based on photographic evidence might reveal for the first time, quite different stories, incidents, memories, attitudes and cross-cultural relationships." ${ }^{173}$

Yet with few exceptions, historical scholarship has failed to seize upon the photograph. According to White, historians have not "exploited the possibilities of using images as a principal medium of discursive representation, using verbal commentary only diacritically, that is to say, to direct attention to, specify, and emphasize a meaning conveyable by visual means

171 Philipp Muller, "Understanding History: Hermeneutics and Source-Criticism in Historical Scholarship," in Reading Primary Sources: The Interpretation of Texts from $19^{\text {th }}$ and $20^{\text {th }}$-Century History, ed. Miriam Dobson and Benjamin Ziemann (Abingdon, Routledge, 2009), 23.

${ }^{172}$ Katharine Martinez, "Imaging the Past: Historians, Visual Images and the Contested Definition of History," Visual Resources 11, no. 1 (1995): 24.

${ }^{173}$ Max Quanchi, "Visual Histories and Photographic Evidence," The Journal of Pacific History 41, no.2 (2006): 165. doi:0.1080/00223340600826052. 
alone." ${ }^{\prime 14}$ Rather than considering photographs as documents capable of investigation and analysis, historians have used them primarily as illustrations. Writing in 1995, Martinez summarizes the current state of image usage by academic historians:

In the world of academia, few historians consult images in their research, and when images appear in scholarly history publications in most cases the images play a secondary role to textual research materials in supporting an argument. Furthermore, the placement of images within illustrated scholarly history books, the quality of the reproductions, and the captions in most cases suggest that images may have been an afterthought, added hurriedly at the last minute to improve the attractiveness of the book once it left the publisher's hands. Why are academic historians so uncomfortable using visual images as evidence? ${ }^{175}$

While empirical research on image use by historians is only beginning to emerge, anecdotal evidence suggests that not much has changed nearly two decades later. Januarius claims that historians still are not using images in their research, an argument made all the more confounding given the increased access to digital images through both digitization and personal digital camera use. ${ }^{176}$ In my own survey of leading academic history journals, I found very little mention of possible methods and approaches and very few actual employing them in historical argument. What are the conceptual challenges and obstacles in using photographs as the basis for historical understanding? Why are historians remiss to use photographs as visual evidence?

\subsubsection{How historians approach photographs as visual evidence}

Historians' epistemological assumptions play a significant role in how they interpret source materials. ${ }^{177}$ In his article "Evidence Revisited: Interpreting Historical Materials in Sport

\footnotetext{
${ }^{174}$ Hayden White, “Historiography and Historiphoty,” The American Historical Review 93, no. 5 (1988): 1194.

${ }^{175}$ Martinez, "Imaging the Past," 22.

176 Joeri Januarius, "Picturing the Everyday Life of Limburg Miners: Photographs as a Historical Source," International Review of Social History 53, no.2 (2008): 294. doi:10.1017/S0020859008003453.

${ }^{177}$ Peter Stearns and Jan Lewis, An Emotional History of the U.S. (New York: NYU Press, 1998$), 1$.
} 
History," Douglas Booth outlines three epistemological approaches and how historians might apply them in the study of historical visual materials. Reconstructionists see photographs as prima facie evidence or visual facts. ${ }^{178}$ Constructionists, on the other hand, think about the historical circumstances in which the photograph was produced, using theory to position the understanding of materials as evidence. For example, constructionists might describe the manner in which a photograph was received by drawing on theoretical frameworks.

Deconstructionists also frame their interpretations in theory, but see photographs and their reception as mediated. For deconstructionists, it is the influence of cultural and social subjectivities that make photographs "ambiguous texts rather than accurate records of 'the truth." $" 179$

In lieu of offering prescriptive approaches to dealing with visual materials, historians have grappled with the problems of interpreting and evaluating visual forms. Such concerns mirror traditional historiographical concern with the accuracy and validity of source materials. In an American Historical Review forum, Hayden White considers new possibilities in how historians can represent truthfulness and accuracy using visual materials. In what he terms, "historiophoty" - or the representation of historical phenomena through visual discourse - White argues for the use of visual materials as demonstrations for types of historical processes. As White explains, "The veracity of the representation hinges on the question of the likelihood of this type of cause-and-effect." 180 White's emphasis on typification allows him to explore the

\footnotetext{
${ }^{178}$ Douglas Booth, "Evidence Revisited: Interpreting Historical Materials in Sport History," Rethinking History 9 , no.4 (2005): 475. doi:10.1080/13642520500307990.

${ }^{179}$ John Bale, “Capturing 'The African' Body? Visual Images and 'Imaginative Sports,"” Journal of Sport History 25, no. 2 (1998): 235-236.

${ }^{180}$ Hayden White, "Historiography and Historiphoty," 1193.
} 
possibilities and benefits of new kinds of characterizations in historical representations, while at the same bringing into question the validity of traditional methods of representation and analysis. Julia Adeney Thomas claims that the complex nature of perception (what she calls the "evidence of sight") prohibits historians' from ever accurately using photographs to interpret the past. ${ }^{181}$ She describes two concepts, recognition and excavation, as the two principle approaches used by historians in dealing with photographic materials. Recognition is a cognitive mode of perception used to position historians' own perceptions of the past, while excavation seeks to recognize the discursive functions bound up in the processes of knowledge production and interpretation.

In her argument, Thomas describes three primary contrasts between the two methods of use. The first difference involves how the act of likeness functions in recognition, as opposed to how contiguity functions in excavation. Recognition, for instance, relies on similarity and difference in photographs to enable the non-discursive perception of likeness, which in turn establishes something in the past. Excavation, on the other hand, contextualizes the photograph within a discursive system, using its material presence to recover the historical system which produced it.

The second difference between the two interpretive processes involves whether one chooses to substitute or reconstruct. To recognize is to substitute through the condition of likeness. In other words, one can point to a photograph and claim that it is "my mother" or "the Civil War," an event or thing that has transpired. We recognize the existence of the photograph as substitute for something else. Similarly, it is only through the excavation of the discursive systems which produced a thing that one can understand how and why that thing exists. As

${ }^{181}$ Julia Adeney Thomas, “The Evidence of Sight," History and Theory 48 (2009): 151-168. 
Thomas explains, "This system, the regularities governing the photograph's emergence as a meaningful object, may exist at some distance from us, an important distance when we look at photographs as historical evidence." ${ }^{182}$

The third contrast is in the interaction with the photograph itself. In the process of recognition, a relationship between two temporalities is cemented. The past and the present intersect in the act of "seeing" the photograph. Excavation preserves the past from the present, through the acknowledgement of the existence of separate discursive formations. In this conception of excavation, the photograph may show the particularities of a past that are distinct from the material presence of the photograph in the "here and now."

Thomas claims that most historians employ one of these two approaches in their treatment of historical photographs. However, she argues that both approaches diminish the ability of photographs to act as evidence. For Thomas, recognition is problematic because it anchors understanding in the already known. We can learn nothing new from the photograph itself and can ask it no new questions. By perceiving it within the frame of likeness (through the process of difference and similarity), we are already viewing it within established territories. Thus, she concludes, "My understanding will always be limited to what I already know and feel; from this approach I cannot achieve a new, articulated, critical grasp of the meaning of the past." 183 It is through the strategy of recognition, Thomas argues, that historians are fooled into thinking they can embrace past realities through the interpretation of photographs.

Excavation as a strategy posits the photograph in an equally complex place for the historian. This approach relies on explicating the context surrounding the production and

\footnotetext{
${ }^{182}$ Ibid, 154.

${ }^{183}$ Ibid, 158.
} 
consumption, of the photograph. Thomas shows the technique in her analysis of an image of two Japanese street children from 1946. Researching a popular image used by historians to signify the "reality" of postwar Japan, Thomas uncovers a debate in an amateur photography magazine from the same time period that calls into question the realities shown in the photograph's depiction. Thomas argues that such an approach is ultimately more capable of showing the past as it really was, to borrow from Ranke's famous dictum.

Elizabeth Edwards advocates using a material culture framework for understanding the function of photographs in discourse. ${ }^{184}$ A trained historian, Edwards also draws prominently from visual anthropology and cultural studies in crafting her theoretical approach. She describes two forms of materiality that can be found in photographs. Both the physical construction of the photograph (the type of print, the use of specific toning) and the form in which it is presented (albums, mounts) reveal intentions and specificities that are, she argues, seldom random. ${ }^{185}$ If one thinks about photographs as cultural artifacts, then one can begin to see their performative aspects and participation in discourse. As Edwards argues, "Understanding the saliency of these entwined practices for those involved is a way of exploring the potential of photographs as historical evidence, and is also a means to investigate the forms through which historical imagination might be made possible and be experienced." 186 As a means for examining what is often obscured by history's intense obsession with metanarratives, photographs can provide a material basis from which to examine historical and social processes at work.

\footnotetext{
${ }^{184}$ Elizabeth Edwards and Janice Hart, Photographs Objects Histories: On the Materiality of Images (New York: Routledge, 2004); Elizabeth Edwards, "Photography and the Material Performance of the Past," History and Theory 48, no.4 (2009): 130-150.

${ }^{185}$ Edwards, Photographs Objects Histories, 3.

${ }^{186}$ Edwards, "Photography and the Material Performance of the Past," 131.
} 


\subsubsection{Methods for analyzing historical photographs}

While discussions of theoretical frameworks abound, the academic historical literature rarely demonstrates empirical methods for analyzing historical photographs. An exception can be found in critiques of pedagogical settings. Louis Masur argues that history textbooks treat images indifferently, rarely given the proper context or reproduction necessary to be taken seriously as historical sources. ${ }^{187}$ He uses one particular photograph, of a slave named Gordon with severe lashings on his back, to demonstrate how the image can be read historically. He compares the photograph with a wood engraving of the same portrait, asking the viewer to consider the differences between the two. For example, (how) does the difference in format impact their reception? The image was circulated in Harpers Weekly, a publication with a known white, middle-class, educated readership, on July $4^{\text {th }}, 1863$ with the caption “A Typical Slave." The captions only appeared in certain reproductions of the image. Does its inclusion suggest evidence about the anti-slavery leanings of the readership? All of these bits of information provide additional context, allowing the viewer to "comprehend this image as history and to read it without losing sight of Gordon." 188

Allen and Felton argue that photographs present opportunities for engaging students in new ways. ${ }^{189}$ They recommend a series of chronological steps that teachers should employ when introducing photographs as historical evidence. First, they advocate presenting photographs with as much context (including the time period) as possible to help orient students. Teachers should ask students narrowly-focused questions about what information the photograph conveys in an

\footnotetext{
${ }^{187}$ Louis Masur, "Pictures Have Now Become a Necessity": The Use of Images in American History Textbooks," The Journal of American History 84, no.4 (1998):1409-1424.

188 Ibid, 1419.

${ }^{189}$ Rodney Allen and Randall Felton, "Photographs as Historical Documents," OAH Magazine of History 5, no.3 (1991): 7-12.
} 
effort to elicit hunches or hypotheses. Third, students should collectively describe all the entities (people, objects) in the photograph. Students should also offer observations that attempt to show contrasts or similarities to the present day. Teachers should probe students to make inferences from their observations, using them as data points. Finally, teachers and students should consult their textbooks and other materials to "confirm hunches." Such instructional approaches, the authors argue, help develop and foster necessary competencies in an increasingly mediadominated age. They prove beneficial for students needing to engage critically with visual materials.

\subsubsection{Case studies: Historians using photographs as visual evidence}

Histories of photography have revealed that photographs were often used as tools in varying social and cultural contexts. Terence Ranger examines one particular African township, Bulawayo, during the 1930s-1960s to show the extent to which photographs served as social capital in the community. ${ }^{190}$ In particular, the romantic pursuits of township men were bolstered by the act of being photographed; according to one male interviewee, "I used to send some of my photographs to a number of girls in my home rural area. Some just accepted my love proposals after receiving my very nice photographs. So photographs were an important tool in my love proposals." ${ }^{191}$ Perhaps more interestingly for social historians is the change in photographic subject matter that happened in the African press. Ranger relates this to the larger societal changes that saw women getting more education and becoming more independent, resulting in what he terms "a readiness to be seen." 192

\footnotetext{
190 Terence Ranger, "Pictures Must Prevail: Sex and the Social History of African Photography in Bulawayo, 19301960," Visual History 27 (2001): 261-266.

${ }^{191}$ Ibid, 264.

192 Ibid, 265.
} 
In Nicole Hudgins' study of family photographic collections from the late $19^{\text {th }}$ and early $20^{\text {th }}$ century in two European towns, she shows how photographs served a unique function for working class families. ${ }^{193}$ She cites the singular example of working class boys in Manchester England who celebrated newly acquired or recently completed apprenticeships by obtaining studio portraits. In another example, Hudgins looks at a family portrait from Lille, France taken by a father of his young children. The photograph depicts a playful scene, with the youngest daughter laughing at the camera while her sister and brother draw cartoonish figures on a barn behind them. Such images, argues Hudgins, work to debunk the "common view of the northern French family, wherein the bread-winning father was perennially absent and uninvolved with the children." ${ }^{194}$ Hudgins concludes that the accessibility of snapshot photography and the resulting proliferation of family portraiture taken across all classes made the medium of photography the "instrument of a modern, democratized family consciousness." 195

By combining historical accounts with family photographs, Hudgins formulates new claims about the embrace of individuality by working class families. She uses the marriage portrait and related archival materials of two Lancashire cotton mill workers, Joseph and Jane Robinson, as examples to argue against the conception popularized by cultural historians that working class families always sought to emulate the bourgeois. Instead, Hudgins postulates that “the Robinsons' simple clothes (hand-made at home, according to archival notes), relaxed pose, and easy expressions give no evidence of such photographic behavior." 196

\footnotetext{
${ }^{193}$ Nicole Hudgins, “A Historical Approach to Family Photography: Class and Individuality in Manchester and Lille, 1850-1914," Journal of Social History 43, no. 3 (2010): 559-586. doi:10.1353/jsh.0.0298. 
Historians have also seen how photographs were used to legitimize and perpetuate certain cultural ideals through visual representation. In "Capturing the African Body? Visual Images and Imaginative Sports" the sports historian John Bale shows how stereotypes of African "Otherness" were cultivated and enforced in visual culture. He analyzes various photographs of Rwandan Tutsis performing the sporting task known as gusimbuka, or high jump, during the first half of the twentieth century. He argues that such visual representation of the Tutsi body in photographs was used to signify differences in power between Europeans and Africans. Bale explains:

In the photographs considered here, the European tended to be placed in a posed, central position, framed not only by the edge of the picture but also by the rectangle of the high jump equipment (seemingly emerging from the portals of Africa) with clear evidence of the African Other (spectators) in the background. There is a visual hierarchy (which symbolizes the world system) in which the Europeans are at the center with the Africans occupying various degrees of peripherality, simulating a map of the colonial world system. ${ }^{197}$

Bale's direct reading of the Tutsi body as represented in the photographic image proves a rarity among academic historians who are less likely than other disciplines (for example, art history or anthropology) to interpret photographs in literal terms. Bale's analysis reveals how photographs of this act always positioned the Tutsi body in one of three modes of representation: appropriation, idealization, and surveillance. ${ }^{198}$

Photographs can also prove useful in understanding social and cultural conditions. In "Picturing the Everyday Lives of Limburg Miners: Photographs as Historical Source," Joeri Januarius examines two photographs from two different mining families to explore domesticity in Belgium's garden cities. Taken in the 1950s in the province of Limburg, the photographs

\footnotetext{
${ }^{197}$ Bale, "Capturing the African Body," 243.

${ }^{198}$ Ibid, 247.
} 
capture two different perspectives on the everyday. The first photograph, a still shot from a promotional film on the work and homes lives of miners, depicts a relaxed family in the midst of various activities in their dining room. The second photograph, also taken in a dining room shows only the wife of a miner and was taken by a family member. According to Januarius' literal reading of the materials, the first photograph can be read as both a "eulogy" to family values and evidence of the family's well-being and comfort level. The relaxed atmosphere shown coupled with the interior decorations combine to create the notion of "a respectable standard of living to which a labourer could reasonably aspire when he began to work for the mine."199 In the second photograph, the furnishings in the dining room testify at a simple level to domestic scenes from everyday life. Januarius argues that a close examination of the objects in the room reveals particularities about the family's domestic life. For example, the placement of objects on top of lace cloths shows a striking “diligence and devotion" while the woman's engagement in domestic work "reveals clues about the changing meaning of space in relation to the presence or otherwise of a member of a family. ${ }^{200}$ While Januarius acknowledges that the two photographs themselves are not enough material to put forth a historical claim, he suggests that the compiling of series of photographs over time could amount to substantial visible evidence for understanding the material culture and practices of everyday life in Belgium's garden cities.

In his photo exposition on modern-day Venice, Jonathan Walker uses the production and display of photographs in a published journal article to present a historicized vision of the city. ${ }^{201}$ The pairing of particular images on a double page spread, the layout of the pages themselves, and

\footnotetext{
199 Januarius, "Picturing the Everyday Lives of Miners," 308.

${ }^{200}$ Ibid, 311.

${ }^{201}$ Jonathan Walker, “Let Us Burn the Gondolas: Venice as a Modern City," Rethinking History 15, no.1 (2011): 37 41. doi:10.1080/13642529.2011.546128.
} 
the choice of fonts all exemplify Walker's desire to portray, in his own words, both the timeless, postmodern Venice often represented with its less acknowledged backstage shadow. As he points out, Venice was the first city to be "packaged and prepared for consumption in the form of visual souvenirs." ${ }^{202}$ He understands the production of such visual forms as a means to constrain perceptions that the city was, in actuality, anti-modernist.

Walker's approach is unique on several fronts. First, he offers a historical argument in a visual form. Secondly, he uses salt printing and other obsolete technologies to create the photographs used in crafting his argument. He claims to use such techniques because they "stand for a particular historical moment."203 Indeed, his approach is driven by a desire to confront the modernist vision of the city as timeless spectacle. However, it also reflects the photograph's ability to act in dual capacities, made visible by tracing and interpreting its material practices. His essay simultaneously offers a reflection of a particular past and an active critique of its representation.

\subsubsection{The current state of historical practice: Summary and implications}

The understanding that historical discourse itself is a constructed representation - aptly reflected in David Lowenthal's invocation of the phrase "the past is a foreign country" - has important ramifications for historical methods and the use of evidence. In fact, the process of how historians construct narratives from their source materials remains largely unexplained in methodological texts. ${ }^{204}$ Moreover, the technological changes that have so largely influenced modes of knowledge production have in turn shifted the form and construct of source materials

\footnotetext{
202 Ibid, 8.

${ }^{203}$ Ibid, 56.

${ }^{204}$ Berkhofer, "Beyond the Great Story," 29.
} 
and "evidence." More attention needs to be paid toward current historical processes to enable the adaptation of methodologies that both use and reflect such changes. What particular challenges does digitization present to historians and how do these challenges impact the capacity of digital primary sources to act as evidence? Such gaps in knowledge become even more pressing with the acknowledgement that historians cannot write good history from bad evidence but can write bad history from good evidence. ${ }^{205}$

Perhaps most notably, there is little mention of visual approaches to historical sources in both the pedagogical literature and in academic historical literature. The absence of visual methodologies and frameworks presents obstacles to historians seeking models for analysis. Moreover, historians need to learn visual literacy skills in order to evaluate and interpret visual materials accurately. In the current state of the field, historians often lack the necessary tools and understanding to effectively treat photographs as historical sources of visual evidence.

\subsection{The Experience of Information: Methodological Approaches}

“...the text is experienced only as an activity, a production" - Roland Barthes, 1971

While the information practices of historian scholars have been well-documented (see 2.1), the study of how historians evaluate and use primary source materials in the course of their research activities is under-investigated. In the LIS literature, information use is often examined through quantitative measures of access to resources (e.g., transaction logs, web analytics) or qualitative analysis of scholarly preferences for resource access (e.g., are scholars getting

${ }^{205}$ David Henige, Historical Evidence and Argument (Madison: University of Wisconsin Press, 2005), 7. 
materials through print or electronic methods ${ }^{206,207}$ ). One limitation of these approaches is that information use is understood solely as resource access - in other words, as the successful outcome of an activity after determining to "do something further" with that resource. Such approaches tell us little about how scholars encounter and "make sense" of primary source materials in their interactions. The experience of these interactions presumably influences how they make decisions about further information use.

In the print world, textual studies helped orient scholars toward the material qualities of literary texts. Both the production and consumption of literary discourse relies on engagement with what Jerome McGann calls the "minute particulars" of the experience. ${ }^{208}$ Yet how does one read and experience the "text" of a digital object? Johanna Drucker suggests that in the digital realm, an object's textuality can be deciphered by attending to its informational qualities. She writes, "To understand what portion of the actual message and meaning a text communicates is challenged, intensified, or lost in this electronic environment, we must ask the basic question 'What constitutes the information of a text?",209 This is one of the guiding questions underlying my dissertation research. For historians interacting with digital archival photographs - how is

\footnotetext{
${ }^{206}$ See Helen Tibbo, "Primarily History in America: How US Historians Search for Primary Materials at the Dawn of the Digital Age," The American Archivist 66, no. 1 (2003): 9-50; Ian Anderson, "Are You Being Served? Historians and the Search for Primary Sources," Archivaria 58 (2004): 88-129; Margaret Stieg-Dalton and Laurie Charnigo, "Historians and Their Information Sources," College and Research Libraries 65 no. 4 (2004): 407; Wendy Duff, Barbara Craig, and Joan Cherry, "Historians Use of Archival Sources: Promises and Pitfalls of the Digital Age," The Public Historian 26, no. 2 (2004): 14.

${ }^{207}$ This tendency could be attributed to the historical theoretical fixation with electronic environments as separate places. For a primer on the meaning and implications of formalizing cyberspace as a separate place from the everyday world, see Julie E. Cohen, "Cyberspace As/And Space," Columbia Law Journal 107, no. 1 (2007): 210256.

${ }^{208}$ In a chapter entitled "Shall These Bones Live?" Jerome McGann describes how relations among various entities constitute the social mileaux of literary works and their interpretation. See: Jerome McGann, The Beauty of Inflections: Literary Investigations in Historical Method and Theory (Oxford: Clarendon Press, 1985).

${ }^{209}$ Johanna Drucker, "Intimations of Immateriality: Graphical Form, Textual Sense, and the Electronic Environment" in Reimagining Textuality: Textual Studies in the Late Age of Print, ed. Elizabeth Bergmann Loizeaux and Neil Fraistat (Madison: University of Wisconsin, Press), 152.
} 
visual information constituted in their experiences? What material practices provoke historians to "make sense" of visual materials in their interactions? How do these practices impact their decision to use (or not to use) digitized photographs?

This section will conceptualize information as a phenomenon that emerges in specific contexts of use. I contend that we cannot understand what information is without understanding how information does. First, I explore how information has been conceived in the LIS literature, with particular attention to its material practices. I situate these conceptions within a larger critique about information use models and digital environments. Finally, I review theoretical approaches and frameworks which embrace experiential, embodied approaches toward exploring interactions with visual information. By studying the experiences of historians interpreting and evaluating digitized photographs, I argue that we can learn more about the factors that make those experiences meaningful.

\subsubsection{Information as material practice: A short history}

In the library and information science (LIS) community, the qualities of what make something "information" have been underexplored. ${ }^{210}$ The discipline tends to defer to a larger framework concerned with behavior around information, rather than defining concepts, attributes, or material properties of information. Problem-oriented empirical work is at the heart of the discipline's research focus, with an emphasis on operationalized investigations of behaviors surrounding the management of information.

Notable exceptions include the work done by early French documentalist Suzanne Briet, who was one of the first theoreticians in the field of information science to directly reference

\footnotetext{
${ }^{210}$ For a conceptual analysis of the term "information" in the discipline of library and information science, see: Jonathan Furner, "Information studies without information," Library Trends 52, no. 3 (2004): 427-446.
} 
objects as forms of information. Writing in the 1930's, Briet describes four distinct levels at which "documentary objects" participate in the activity of information: they can instruct, explore, diffuse, or organize. For example, an academic journal is a documentary object which uses facts to instruct. Documentary techniques center on two tendencies:

The first is toward an always increasingly abstract and algebraic schematization of documentary elements (catalogs, codes, perforations, classifications through conventionally agreed upon marks). The second is toward a massive extension of "substitutes for lived experiences" (photos, films, television, audio records, radio broadcasting). What words fail to communicate, image and sound try to deliver to all. Documentation, thus understood, is a powerful means for the collectivization of knowledge and ideas. ${ }^{211}$

In this way, objects are imbibed with a sense of agency for performing activities which "make known." Briet defines a document as "any concrete or symbolic indexical sign, preserved or recorded toward the ends of representing, of reconstituting, or of proving a physical or intellectual phenomenon." ${ }^{212}$ Documentation is thus one form of evidence existing in relationship to other forms of evidence, motivated by an intention to make something known. ${ }^{213}$

Arguably the theory of information that has had the strongest influence on the discipline is Claude Shannon and Warren Weaver's model of mathematical communication, also known as Information Theory. ${ }^{214}$ Originally published in 1948, Shannon and Weaver's engineering model

\footnotetext{
${ }^{211}$ Suzanne Briet, What is Documentation?, ed. Ronald E. Day and Laurent Martinet (Lanham, MD: Scarecrow Press, 2006), 31.

${ }^{212}$ Ibid, 10.

${ }^{213}$ There are two different translations of Briet's definition of a document. In the 1997 article "What is a 'Document'?" Michael Buckland cites Briet's definition of a document as beginning with "any physical or symbolic sign..." Interestingly, he goes on to cite a discussion with Ronald Day, who translated the only English version of Briet's full text and provided a markedly different translation. Buckland mentions that Day suggests Briet's use of the word "indexical" demonstrates her belief that objects achieve documentary status when "placed in an organized, meaningful relationship with other evidence." For the full discussion, see: Michael Buckland, "What Is A 'Document'?" Journal of the American Society for Information Science 48, no. 9 (1997): 806.

${ }^{214}$ In 1984, Professor Pranas Zunde compiled a "selected" bibliography of 440 publications that reference the application of information theory to information science. See: Pranas Zunde, "Selected Bibliography on Information
} 
is primarily concerned with effective message transmission across an information channel and factors that may impact reception (such as channel capacity, noisiness, or degrees of uncertainty). The model is explicit about not incorporating meaning into a definition of information, instead claiming that the "semantic aspects of communication are irrelevant to the engineering problem." 215

In his investigation of information theory, the British scientist Donald MacKay offers a useful definition of information which benefits the current discussion. MacKay argues that Shannon's famous mathematical model of communication, adapted heavily by system theorists during the 1950s and beyond, is primarily concerned with the efficiency of information transfer between two points, rather than the meaning of the message transferred. ${ }^{216}$ MacKay rightly recognizes the semantic components of information-making as part of the information transfer process: "The mechanical energy of a message must be sufficient to do the mechanical job that eventually resets the brain-levels; but the selective job, of determining which levels shall move, depends on the form of the message, and on the state of your brain before you hear it." ${ }^{217}$ For MacKay, perceiving a message is made possible by an internal state of conditional readiness. Importantly, he argues that while all stimuli are capable of being received, only those which evoke a matching response are perceived. This distinction is critical to understanding how perception itself is embodied in the activity of information-making, or, as MacKay puts it, the

Theory Applications to Information Science and Related Subject Areas," Information Processing and Management 20, no.3 (1984):417-497.

${ }^{215}$ Claude E. Shannon, “The Mathematical Theory of Communication,” The Bell System Technical Journal 27 (1948): 379-423, 623-56.

${ }^{216}$ For more discussion on Donald MacKay's semantic information theory, see: John Soloski, "Meaning and Information: A Perspective on Donald M. MacKay," Journal of Communication Inquiry 2, no. 2 (1977): 51-59.

${ }^{217}$ Donald MacKay, Information, Mechanism and Meaning (Cambridge: MIT Press, 1969), 24. 
"activity of knowing is an activity of internal adaptive response." ${ }^{218}$ Perhaps most importantly, it highlights the dynamic nature of meaning as it is encapsulated in information transferred.

During the 1980s and 1990, Michael Buckland was the preeminent information scientist writing on the ambiguity of the term "information." Given the right set of conditions, Buckland argues that any "thing" can be perceived as informative or evidential. Invoking Briet's earlier assertion that a captured antelope in a zoo becomes a document, he writes:

On this view objects are not ordinarily documents but become so if they are processed for informational purposes. A wild antelope would not be a document, but a captured specimen of a newly discovered species that was being studied, described, and exhibited in a zoo would not only have become a document, but the "catalogued antelope is a primary document and other documents are secondary and derived." 219

Rather than limit ourselves simply to documents, Buckland suggests expanding what he calls the "species of information" to include objects and events so that we can better understand the concepts underlying them. According to Buckland, "Pursuing the notion of information as evidence, as things from which one becomes informed, we can examine more specifically what sorts of things this might include." ${ }^{220}$ In other words, by redirecting our attention to what makes various things "informative" we can observe how situations and context affect the process of making meaning.

Robert S. Taylor proposes that the value of information can best be understood in the context of use, through the development of user-driven models and approaches. ${ }^{221}$ Taylor puts forth a conceptual framework for an "information use environment" which describes "the set of

\footnotetext{
${ }^{218}$ Ibid, 61.

${ }^{219}$ Michael Buckland, "Information as Thing," Journal of the American Society for Information Science 42, no. 5 (1991): 355 .

${ }^{220}$ Ibid, 353.

${ }^{221}$ Robert Taylor, Value-added Processes in Information Systems (New Jersey: Ablex Publishing Corporation, 1986), 14.
} 
those elements that (a) affect the flow and use of information messages into, within, and out of any defined entity; and (b) determine the criteria by which the value of information messages will be judged." 222 For Taylor, information is thus always situated contextually in both its production and consumption. It cannot be quantified systematically, but rather assessed through an understanding of the meaning attached to the value established in the context of use. Taylor explains, "Data become information when we establish relationships among data. That is information is not just more labels. It is the structure and relations among data, and most important, the rules and conditions used to establish those relations."223

A similar philosophical orientation underlies the work of Birger Hjorland, who argues in favor of socio-cognitive approaches to information. Hjorland regards information as an emergent phenomenon, arising in specific contexts and trigged by specific mechanisms. He introduces the concept of domain analysis, which stipulates that the meaning of a term can only be understood within the context (or domain) in which it appears. Hjorland uses gold as an example to demonstrate how a single thing can have a variety of meanings depending on the context in which it is interpreted. Chemists might define gold as having certain properties and attributes that differ from how economists would define it. ${ }^{224}$ Thus, the production of information can only be understood by examining the discourse of the communities and organizational structures in which it is generated.

Paul Solomon suggests that the study of information use should shift towards investigating the processes of how something becomes information - what he terms "information

\footnotetext{
222 Robert Taylor, "Information Use Environments," in Progress in Communication Sciences Vol. 10, ed. Brenda Dervin and M.J. Voight (Norwood, NJ: Ablex Publishing Corp, 1991), 218.

223 Taylor, Value-added Processes, 7-8.

${ }^{224}$ Birger Hjorland and Hanne Albrechtson, “Toward a New Horizon in Information Science," Journal of the American Society for Information Science and Technology 46, no.6 (1995): 413.
} 
discovery." Solomon characterizes information to be a socio-technical construction, drawing attention to "engagement, reflection, learning, and action" as dimensions important to understanding when and how information discovery happens. ${ }^{225}$ According to Solomon, an evidence-based understanding of information in context is critical to building effective sociotechnical systems that support information needs and uses.

In their book Interacting with Information, Ann Blandford and Simon Attfield point out that in spite of living in the middle of an information age, "information is only useful when it is interpreted by people and applied in the context of their goals and activities."226 For Blandford and Attfield, it is the mediating factors (often invisible) in both digital and physical environments that present challenges for supporting information work. Indeed, evolving information needs what the authors term "an information journey" - are complex sites of activity:

There are many aspects of the situation, including the physical situation in which interaction takes place, the ecology of resources to which individuals have access, the social structures within which people interact with information, the individual knowledge and skills people bring, and temporal aspects, through which the above can dynamically evolve and change. ${ }^{227}$

The authors refer to these sites as spaces of situated information interactions. Information interactions take place within contexts that are "physical, social, and activity-centered" and can overlap situationally. ${ }^{228}$ The value in studying spaces of information interactions becomes clear when considering how often we encounter and use information across spatio-temporal dimensions, in a variety of contexts.

\footnotetext{
${ }^{225}$ Paul Solomon, "Discovering Information in Context," Annual Review of Information Science and Technology 36, no.1 (2005): 229.

${ }^{226}$ Ann Blandford and Simon John Attfield, Interacting with Information (San Rafael, California: Morgan \& Claypool, 2010), 2.

${ }^{227}$ Ibid, preface.

${ }^{228}$ Ibid, 2.
} 
Diane Nahl proposes that affective dimensions play a central role in information behavior. She introduces a framework for a "unified theory of information" which charts information reception and use as constant interactional processes at both micro-level and macrolevels of activities. Nahl argues that "functioning in an information setting requires alternating between steps of reception and steps of use in a continuous dynamic flow."229 Information reception processes consist of noticing, appraising, and then evaluating information; the process of information use begins once there is an intention or motivation related to that information. Once information becomes "value-attached", the process of information reception is completed and the "use process of engaging the system can begin.",230

\subsubsection{Information use in digital environments}

Current theoretical frameworks in information use derive much from the application of Shannon and Weaver's model to information behavior research. One problematic result has been a flattened conception of information as a "substance that is transported between the sender and the receiver." 231 This so-called "process-oriented" approach characterizes information as an objective entity in an external reality. ${ }^{232}$ Transmission occurs without specific attention to semantic factors that may hinder or impact reception. Jonathan Furner explains the problem with such a conception:

\footnotetext{
${ }^{229}$ Diane Nahl, "The Centrality of the Affective in Information Behavior," in Information and Emotion: The Emergent Affective Paradigm in Information Behavior Research and Theory, ed. Diane Nahl and Dania Bilal (Medford, New Jersey: American Society for Information Science and Technology, 2007), 9.

${ }^{230}$ Diane Nahl, "Social-Biological Information Technology: An Integrated Conceptual Framework," Journal of the American Society for Information and Technology 58, no. 13 (2007): 2028.

${ }^{231}$ Jens-Erik Mai, "The Quality and Qualities of Information," Journal of the American Society for Information Science and Technology 64, no.4 (2013): 676.

232 Rafael Capurro, "Some Critical Comments on Three Leading Paradigms of Information Science Section, Para.1," Foundations of Information Science: Review and Perspectives.
} 
The conduit metaphor seems to serve only to reify the controversial idea that information is something that can somehow "flow" from one place to another. Such flow would be possible only if messages were inherent properties of signals, rather than separate entities assigned to signals by humans. ${ }^{233}$

The end result is that there is very little qualitative understanding about the material practices that make something information.

In the digital realm, information use has largely been interpreted in terms of successful transmission. Information use in digital environments is most often analyzed through usage statistics (log analysis, web analytic tools) which treat access as a proxy for use, or through citation analysis which tracks the frequency with which an object or collection is referenced in literature. Both of these approaches frame information use solely as the outcome of the process of transfer without qualitative attention to what it means to actually use materials in the context of the collection. ${ }^{234}$ What counts as "information use" here is simply access - the user finds an object using a specific information channel to retrieve their source. ${ }^{235}$ Yet what makes the interaction meaningful? By focusing on the information channel as the marker of information use, the user's material interactions with an object in the information space has been obscured.

The rupture between object and information channel becomes the most evident in digital information environments. Unlike analog media, one cannot look at a digital object in an

\footnotetext{
${ }^{233}$ Jonathan Furner, "Information Studies Without Information,"12.

${ }^{234}$ Molly Bragg's 2011 survey on how libraries were measuring use of digitized primary source materials confirms this tendency. She writes, "Participants raised the point that although the usage statistics provide an idea of how often an item was viewed they do not say anything about how the patron used the resource." See: Molly Bragg, “"There is Always More That Can Be Done': A Survey Investigating Libraries' Measurement of Digitized Primary Source Use," (MA thesis, University of North Carolina Chapel Hill, 2011).

${ }^{235}$ Amanda Spink and Charles Cole argue that in some information behavior research, "information use" is misconstrued. Rather than investigating "user's use of sources of information," studies are actually "asking about the user's use of accessing channels to information sources." See: Amanda Spink and Charles Cole, "Human Information Behavior: Integrating Diverse Approaches and Information Use," Journal of the American Society for Information Science and Technology 57, no. 1 (2006): 28.
} 
interface and see its underlying material properties as it is stored on a physical carrier (e.g. hard drive). In his analysis of new media, Mark Hansen explains the severity of this disjuncture:

Arguably for the first time in history, the technical infrastructure of media is no longer homologous with its surface appearance. As distinct from phonography, where the grooves of a record graphically reproduce the frequency ranges of humanly perceivable sound, and from film, where the inscription of light on a sensitive surface reproduces what is visible to human eye, properly computational media involve no direct correlation between technical storage and human sense perception. What we see on the computer screen (or other interface) and hear on the digital player is not related by visible or sonic analogy to the data that is processed in the computer or digital device. Indeed, as the work of some digital media artists has shown, the same digital data can be output in different registers, yielding very different media experiences. ${ }^{236}$

Whereas Marshall McLuhan theorized that content was united with form in the medium, Hansen's analysis shows that the technical logic of infrastructure (and its material practices) is now distinctly separate from the content it governs. Following on Hansen's example, consider how the physical attributes visible on a vinyl record reflect what is (humanly) audible to the ear. A bass-heavy vinyl record, for example, will have wider grooves in the vinyl because the signal needs more space to move. The groove on a record correlates to its material capacities. One can look at a record and observe, through visible inscriptions on the medium, its functional qualities. $^{237}$

In digital environments, one is no longer able to perceive an object's material attributes simply by looking at the medium itself. Consider the following example. A photograph of a 1928 Ford Model A Phaeton automobile has been digitized (Object A). Perhaps the digitizer wants to brighten the photograph ever so slightly, so she creates another version of the image

\footnotetext{
${ }^{236}$ Mark B. N. Hansen, "New Media," in Critical Terms for Media Studies, ed. W.J.T. Mitchell and Mark B.N. Hansen (Chicago: The University of Chicago Press, 2010), 178-179.

${ }^{237}$ According to a biography of Thomas Edison, the inventor of the phonograph claimed he could "tell a soprano from Basso Baritone or Tenor \& each from another also every instrument in the orchestra when played alone by looking at record thru a microscope." Paul Israel, Edison: A Life of Invention (New York: John Wiley, 1998), 436437.
} 
with adjusted color tones (Object B). If historians interact with the photograph in an online archival collection, it is unlikely that they will be able to ascertain simply by looking at the image in their web browsers that it has been altered. Only through the use of additional tools (e.g., photo editing software, metadata extractors, hex editors) would they be able to surface the RGB values accounting for the difference in color.

This is one of the central challenges in understanding interactions in digital information spaces - we cannot "look" through an interface at a digital object and perceive its underlying material qualities. Accordingly, as the technical inscriptions which characterize a digital object change, so may the interpretation. A representation in the digital realm is just always that - an amalgamation of algorithms fusing content and form to produce a distinct space of interaction. Nearly forty years ago, the sociologist John McHale wrote:

We are faced... with the emergence of a new and powerful fusion of technological capabilities which not only potentially amplifies our capabilities to deal with our social and physical environment transactions but which by its function as screen, channel and multiplexer of information actually reshapes the information content and perception of society - in ways that our conventional wisdom and traditional institutional means may not be able to foresee, comprehend or effectively control. ${ }^{238}$

It is within this constituted information space, born out of technological fusion, that we must begin our interrogation.

\subsubsection{Theoretical approaches to studying use in digital environments}

Studying how historians experience photographs in online archival research environments necessitates grappling with the uneasy convergence of form, content, interaction, and meaning-making. In the process of digitization, an object changes form and structure in the translation. Accompanying these permutations in character are traces of activity documenting the digital object's lifecycle, encapsulating both the object and related provenance information in the

${ }^{238}$ John McHale, The Changing Information Environment (Boulder: Westview Press, 1976), 16-17. 
same representational space. ${ }^{239}$ The degree to which digitization changes the experience of interactions with primary source materials has received limited empirical attention. ${ }^{240}$

Born-digital objects also present complexities for access, interpretation and use. For archives acquiring born-digital materials, modifying existing workflow practices to accommodate processing needs presents technical, administrative, and intellectual challenges. Very little is known about the types of metadata users will require in order to make sense of their interactions with born-digital objects. Currently, few digital archives provide item-level access to born-digital materials, though this is likely to change.

\section{Performative materiality}

The notion that digital objects are constituted by a kind of materiality is not itself new; neither is the claim that materiality is an emergent property born out of interactions among entities. In her book Writing Machines, N. Katherine Hayles writes, "In the broadest sense, materiality emerges from the dynamic interplay between the richness of a physically robust world and human intelligence as it crafts this physicality to create meaning." ${ }^{241}$. What remains underexplored, however, is that interplay as it happens-individual experiences of interactions within digital information space and how those interactions constitute the experience of the thing itself under study. Heidegger famously asserted that "a hammer's being reveals itself by its handiness; handiness is discovered in the act of hammering." ${ }^{242}$ Yet how does one study this

\footnotetext{
${ }^{239}$ For a brief treatise on the enduring essences of digital life, see Bruno Latour, "Beware your Imagination Leaves Traces," Times Higher Education Literary Supplement, April 6, 2007.

${ }^{240}$ Some notable exceptions include: Bradley Taylor, "The Effect of Surrogation on Viewer Response to Expressional Qualities in Works of Art," (PhD diss., University of Michigan, 2001); Margaret L. Hedstrom, Christopher A Lee, Judith S Olson, and Clifford A Lampe, “'The Old Version Flickers More': Digital Preservation from the User's Perspective," The American Archivist 69 (2006): 159-187.

${ }^{241}$ N. Katherine Hayles, Writing Machines (Cambridge: MIT Press, 2002), 33.

${ }^{242}$ Martin Heidegger, Being and Time (Albany: SUNY Press, 1973), 65.
} 
enactment? Particularly in the context of the digital environment, where numerous clicks and visits to websites offer only partial understanding of information behavior?

Johanna Drucker has proposed that we employ a performative model as a means of understanding meaning-making in digital objects at play. ${ }^{243}$ For Drucker, mechanistic models of digital materiality do not take into account the qualities of what she terms "digital flux" - an ecosystem which is constituted by "contingent fields, flows, and relations." ${ }^{\text {244 }}$ These relationships and transactions function as probability conditions which may (or may not) provoke a space in which information as a process of meaning-making can emerge.

Drucker suggests we focus on the interface as a critical point of intervention. As a mediating factor, interface design should reimagine itself as a space of interpretation with uncertainty and abstractness as data points. She advocates for a shift away from homogenous digital information spaces constituted by "things and entities" toward an "event space of interpretative activity. ${ }^{245}$ Drucker hints at the idea that perception is one of the factors impacting the process of meaning-making, but stops short of formalizing its role in the process. In her invocation of constructivist epistemology, she reminds us that meaning and interpretation always emerge from a confluence of mediating factors. What she does not attempt in her analysis is an in-depth characterization of the thing (or things) that are provoked - in other words, a more specific grounding of what and how those material conditions constitute interactions which produce meaning in the digital information space.

\footnotetext{
${ }^{243}$ Johanna Drucker, "Performative Materiality and Theoretical Approaches to Interface," Digital Humanities Quarterly 7, no.1 (2013).

${ }^{244}$ Ibid, 12.

${ }^{245}$ Ibid, 17.
} 


\section{Phenomenography}

Phenomenography is a qualitative research approach emerging from the interpretivist paradigm. It was originally developed in Sweden during the 1970s by a group of researchers in the Department of Education at Goteborg University, led by Ference Marton, as they conducted research on variations in student learning. The term was coined in 1979 and appeared in publication for the first time in $1981 .^{246}$

Phenomenography is concerned with the "description, analysis, and understanding of experiences. ${ }^{247}$ In particular, phenomenonographic research aims to reveal the qualitatively subjectively different ways that people experience various phenomenon. A distinctive feature is an orientation towards "second-order perspectives," which emphasize how participants describe their experiences in the world. In contrast, first-order perspectives are concerned with exploring existing mental models to explain behavior. Importantly, phenomenography is considered to be a relational approach which posits that "the way in which the subject experiences the object forms a relation between the two."248 Capturing the various ways in which people experience meaning is the central aim of phenomenographical research. There are two components to the experiences of a phenomenon: referential (what is being experienced) and structural (how an individual thinks about the experience). ${ }^{249}$

\footnotetext{
${ }^{246}$ Ference Marton, "Phenomenography - Describing Conceptions of the World Around Us," Instructional Science 10 (1981): 177-198.

${ }^{247}$ Ibid, 177.

${ }^{248}$ Louise Limberg, "Phenomenography: A Relational Approach to Research on Information Needs, Seeking, and Use," The New Review of Information Behaviour Research 4, no.1 (2003): 54.

${ }^{249}$ Marian Smith, "Young People: A Phenomenographic Investigation into the Ways They Experience Information" (PhD diss., Loughborough University, 2010): 80.
} 
Christine Bruce used phenomenography to reveal variations in the ways information is perceived. In her study of information literacy among higher educators, she uncovered three different conceptualizations of information (as objective and part of the external environment, subjective and internal to the individual, or transformational) among descriptive categories. ${ }^{250}$ The nuances of her research findings offer critical insight into how variances in a phenomenon of interest (in this case, information literacy) can be influenced by differences in underlying conceptualizations.

Phenomenographic research also helps to reveal the social contexts in which human concepts are formed, addressing a "decisive weakness of the cognitive view of LIS." 251 In his critique of the cognitive perspective, Birger Hjorland argues that people are expected to "react to something in a specific, mechanical way without considering the culturally determined meanings and without considering the different goals and values of the meanings of the documents."252 Experience is not a fixed cognitive structure or mental map that can be empirically observed. Accordingly, phenomenographers seek to show how there are qualitatively different "ways people experience, conceive, perceive and understand various phenomena in, and aspects of, the world around them."253

\section{Digital hermeneutics}

Another conceptual framework for exploring how digital environments impact interpretation and meaning-making is digital hermeneutics. Rafael Capurro defines digital

\footnotetext{
${ }^{250}$ Christine Bruce, The Seven Faces of Information Literacy (Adelaide: Auslib Press, 1997).

${ }^{251}$ Limberg, "Phenomenonography," 53.

${ }^{252}$ Birger Hjørland, "Domain Analysis in Information Science: Eleven Approaches - Traditional as Well as Innovative," Journal of Documentation 58, no. 4 (2002): 431.

${ }^{253}$ Marton, "Phenomenography: A Research Approach to Investigating Different Understandings,” 31.
} 
hermeneutics as "the study of social systems of interpretation and social construction of meaning based on the Internet."254 With a nod towards Don Ihde's formulation of "material hermeneutics ${ } 255$ Capurro argues that the rise of the internet and other networked technologies have transformed our spatio-temporal experience such that an ontological hermeneutic shift is required. In this new realm of networked existence, "things are (understood) as far as we are able to digitize them. ${ }^{, 256}$ Meaning-making and communication processes emerge as embodied practices within an ever-changing set of relationships, uses, and contexts. Digital hermeneutics is thus an attempt at theorizing processes of interpretation in digital information space, where "all receivers of mass media messages into potential messengers beyond the one-to-one technology of the telephone." ${ }^{257}$

\section{Media archaeology}

Media archaeology also offers a useful theoretical frame for exploring interactions in digital information spaces. The German media archaeologist Wolfgang Ernst explains that "media archaeologically, the medium primarily means measurement." 258 For Ernst, a media archaeological approach helps reveal aspects of media that are only ever observable from an algorithmic perspective - what he calls their "nondiscursive infrastructure." ${ }^{.259}$ His approach

\footnotetext{
${ }^{254}$ Rafael Capurro, "Digital Hermeneutics: An Outline.” AI \& Society 25, no.1 (2009): 35-42, doi:10.1007/s00146009-0255-9.

${ }^{255}$ According to Ihde, material hermeneutics "is a technique whereby things - materialities - are given a voice." See: Doh Ihde, "More Material Heremenutics," Yearbook of the Institute for Advanced Study on Science, Technology and Society (Graz: Profil Verlag, 2004), 341-50.

${ }^{256}$ Capurro, Digital Hermeneutics, 38.

${ }^{257}$ Ibid, 39.

${ }^{258}$ Wolfgang Ernst, "Distory," in Digital Memory and the Archive, ed. Jussi Parikka (Minneapolis: University of Minnesota Press, 2013), 165.

${ }^{259}$ Wolfgang Ernst, "Media Archaeography," in Digital Memory and the Archive, ed. Jussi Parikka (Minneapolis: University of Minnesota Press, 2013), 57-58.
} 
purposively privileges the "recording of the story" rather than any narrative tendencies because "machines do not tell stories, they count."260 Thus, Ernst's focus on the underlying technical processes of media (signal processing, operations, executing, and synchronization) offers an epistemological alternative to historiographical cultural time. ${ }^{261}$

A media archaeological approach positions the digital artifact and its attendant properties as always already emergent activated in/by the process of being calculated. In Ernst's media archaeological framework, artifacts are activated from undead to alive when their signal is received. He gives the example of the Volksempfanger, a German radio receiver built during the 1930 s, which is able to receive radio programs today because "the stable technological infrastructure of broadcasting media is still in operation." 262 It is the successful transaction of radio signals received by existing infrastructure which in turn activates an information space - or what Ernst calls a "micro-temporal space."

The media archaeological perspective helps us to interrogate how technological capacity influences the space(s) of interaction. For Ernst and other media archaeologists, the importance of this space is that it reveals the underlying technicalities of media as constituted in specific mathematical moments. Insofar as each representation of a digital artifact occupies a distinct space for interpretation, it is the interactions within these spaces that perform and co-constitute processes of information. Importantly, Ernst does not refer to the materiality of artifacts as being mathematical - only the time-critical processes which constitute their coming into being.

\footnotetext{
${ }^{260}$ Wolfgang Ernst, "Media Archaeology as Method of Re/Search in Parallel Lines" (lecture notes, transmediale.12 "In/compatible" Symposium, Berlin, Germany, February 5, 2012), https://www.medienwissenschaft.huberlin.de/de/medienwissenschaft/medientheorien/ernst-in-english/pdf/transmedial12kurz.pdf.

${ }^{261}$ For critiques on the problems with traditional narrative historiography, see: Robert Berkhofer, Beyond The Great Story; Hayden White, Metahistory; Arthur Danto, Narration and Knowledge.

${ }^{262}$ Ernst, "Media Archaeography, 56-57.
} 
In digital information environments, the capacities of the medium are still intensely correlated with the activity of perception. The difference is that humans cannot observe or perceive digital signals solely through our sensorimotor capacities. Thus, we are dependent on a variety of multimodal tools and approaches to interrogate the interactions that constitute digital representations. Unlike a vinyl record, one cannot look at a digital photograph and actively perceive its underlying techno-material basis simply by looking at it. A media archaeological gaze enables us to gain access to this seemingly invisible material substrate. Ernst explains:

Although a Greek vase can be interpreted by simply being looked at, a radio or computer does not reveal its essence by monumentally being there but only when being processed by electromagnetic waves or calculating processes. If a radio from a museum collection is reactivated to play broadcast channels of the present, this changes its status: it is not a historical object anymore but actively generates sensual and informational presences. ${ }^{263}$

By attending to the non-cultural, non-semantic transactions of media in operation, Ernst proposes that we can better investigate technical differences.

\subsubsection{Information use as emergent experience}

This chapter argues that the study of information use in digital environments would greatly benefit from the application of theoretical approaches that emphasize the experience of the phenomenon. In particular, I suggest that the concept of information in digital objects emerges in specific moments of use, dependent on contextual factors. As such, the importance of investigating how a digital object is received and subsequently used depends necessarily on investigating an individual's experience with that object.

Drucker's notion of "performative materiality" suggests that knowledge creation is the result of a series of provocations and probabilities. Yet what do these provocations look like? What are the sets of material conditions at work? While Drucker does suggest designing flexible

${ }^{263}$ Wolfgang Ernst, "Media Archaeography," 57-58. 
interfaces to accommodate fluid interpretations, she stops short of outlining what an interrogation into these spaces might look like. An investigation into those conditions might include attempts to record specific environmental factors in a setting in which a historian interacted with a digital photograph, or perhaps an in-depth discussion with the historian about his/her disciplinary background. These investigations would certainly prove fruitful for providing a rich account of ecological factors of influence, by revealing the material practices that contribute to the provocations of information as it is performed.

Similarly, media archaeology provides a useful theoretical basis for understanding how technical specificities of media impact experience. In particular, media archaeological approaches emphasize focusing on the operational processes that help constitute interactions. Thus, media archaeology prioritizes and privileges the machine perspective in understanding our experiences with media. A media archaeological approach would prove useful as a conceptual basis for encouraging historians to consider new ways of writing histories that "show" rather than "describe" (in Ernst's terms). Such an approach might be of particular interest for historians concerned with the narrative tendencies at work in the creation of history as "the Great Story" (as discussed by Berkhofer, White, and Danto). An example of a media archaeological approach in operational terms might involve presenting a historian with a digitized photograph both with and without additional EXIF metadata, noting the qualitative differences in how they experienced the object.

Finally, digital hermeneutics provides a useful conceptual framework for understanding practices and processes of interpretation in digital environments. One self-proclaimed example of digital hermeneutics is the Agora Project, which uses semantic event-modelling to embellish access to online cultural heritage objects. In particular, the project aims to facilitate rich 
interpretation of collection objects through associations with related contextual materials. The project team developed a Simple Event Model (SEM) ${ }^{264}$ for collection objects that consisted of four types of event properties: actor, place, time, and type. ${ }^{265}$ In a paper detailing project use cases, the authors show how their model can be used to enhance historical understanding. They describe a specific collection object, a painting of a soldier from the "KNIL" (a division in the Royal Netherlands East Indies Army) stealing a chicken. Using the Agora SEM, a relationship is created between the collection record for the painting and the actor property "KNIL." Users can choose to explore associated pictures or events using the actor property, in effect constructing a "biographical narrative." Thus, multiple pathways for the user are generated through the application of the SEM to the bulk of collection materials. According to Capurro, a flexible design approach is characteristic of digital hermeneutics, which "can look at the whole ("totum") from different perspectives but not at the same time ("non totaliter"'). Whereas scholarship in the digital humanities has helped to conceptualize new ways of thinking about big data and networks, digital hermeneutics suggests that knowledge discovery and interpretation in the $21^{\text {st }}$ century are constituted happenings built from interactions and relationships in the networked environment.

Each of these theoretical approaches provides ample fodder for thinking about information experiences in digital environments. Digital media constitute conditions which require: activation (according to Ernst), provocation (according to Drucker), or new methods for understanding (according to Capurro). These philosophical and epistemological assumptions

\footnotetext{
${ }^{264}$ For an overview of the SEM, see: Willem van Hage, Veronique Malaise, Roxane Segers, Laura Hollink, and Guus Schreiber, "Design and Use of the Simple Event Model (SEM)," Web Semantics: Science, Services and Agents on the World Wide Web 9 (2011): 128-136.

${ }^{265}$ Chiel van den Akker, Susan Legene, Marieke Van Erp, Lora Aroyo, Roxane Segers, Lourens Van der Meij, et. al., "Digital Hermeneutics: Agora and the Online Understanding of Cultural Heritage," ACM Web Science Conference (Koblenz, Germany, June 14-17, 2011).
} 
have provided guidance for the design of this research study. In digital environments, the emergence of an information space produced from the interaction between content and form can become a frame for our inspection. In locating the material traces that contribute to the process of something becoming information, I argue that we can reveal essential characteristics and attributes about information experiences in digital environments. 


\section{CHAPTER 3: METHODOLOGY}

How scholars interact with visual materials is an under-researched area across disciplines, due in part to the inherent complexities in explicating processes of visual understanding. Images are polysemic by nature: meaning is dependent on the viewer and the same image may have multiple interpretations at the same time. ${ }^{266}$ In empirical research, visual materials are most often used as stimulus to elicit further analysis and understanding. ${ }^{267}$ While methods for interpreting and analyzing visual data are increasing in both frequency of use and methodological rigor, the empirical study of scholarly practices related to interpretation and analysis of visual data is severely lacking.

\subsection{Methodological Approach}

The purpose of this case study was to explore how and why historians are using digitized archival photographs in their scholarly pursuits. The main unit of analysis was identified as: " $a$ historian's experiences using digitized photographs as evidence." An embedded unit of analysis, or subunit, was defined as "a historian's specific use of a digitized photograph as evidence in research or instruction." I collected, analyzed, and triangulated multiple sources of data to provide a holistic description of historians' experiences using digitized photographs as forms of evidence. According to Yin, a case study is an "empirical inquiry that investigates a

\footnotetext{
${ }^{266}$ In his essay "Rhetoric of the Image," Roland Barthes notes how images imply a "floating chain of signifieds," some of which are attended to, while others are ignored. See: Roland Barthes and Stephen Heath, Image Music Text (New York: Hill and Wang, 1977), 39.

${ }^{267}$ For a good overview, see: Douglas Harper, “Talking about Pictures: A Case for Photo Elicitation,” Visual Studies 17 (2002): 1.
} 
contemporary phenomenon within its real-life context, especially when the boundaries between phenomenon and context are not clearly evident."268

Data collection consisted of semi-structured, in-depth interviews with self-identified historians who have used digitized photographs in scholarly activities. Artifacts related to each participant's prior use of photographs as forms of evidence were collected for analysis. The use of multiple types of data including examples of historians' specific use helped to facilitate a complete picture of my phenomenon of interest (i.e. historians' experiences using digitized photographs as forms of evidence). During both data collection and analysis, I took memos as a strategy to "trace the thinking of the researcher and help guide a final conceptualization that answers research questions (or related ones) and offers a theory as an explanation for the answers."269

\subsection{Rationale for Methodological Approach}

In this research, I was interested in exploring how and why historians are using digitized photographs as forms of evidence. My goal was to provide an in-depth, holistic understanding of a complex interaction space made up of, but not limited to: digital surrogates of archival objects, user perceptions and attitudes, environmental constraints, and historical training and orientation. ${ }^{270}$ Two theoretical propositions about information and information use informed this research:

\footnotetext{
${ }^{268}$ Robert Yin, Case Study Research: Design and Methods $2^{\text {nd }}$ edition (Thousand Oaks, CA: Sage Publishing, 1994), 13.

${ }^{269}$ W. Newton Suter, Introduction to Educational Research: A Critical Thinking Approach (Thousand Oaks, California: SAGE Publications, 2006), 346.

${ }^{270}$ While Human Computer Interaction (HCI) provides some empirical basis for exploring the role that the interface plays in meaning-making, Jensen and Sogaard argue that the discipline currently lacks theoretical approaches that explore "the dynamicity of the construction of meaning during interaction." Mikkel Jensen and Mads Søgaard. "Refocusing the Contextual Turn: The Forgotten Construction of Meaning at the Interface," in Proceedings of the Third Nordic Conference on Human-Computer Interaction (New York: ACM, 2004), 187.
} 
1) Information is a phenomenon that emerges in specific contexts.

2) Information use is always situated within contextual, ecological, and social practices. As such, we cannot understand the "how" of information use without attending to the practices which constitute the experience.

These propositions are born out of the literature reviewed, and supported by the theoretical approaches discussed in section 2.5.

An embedded case study was selected as the most appropriate methodology for this research. Case studies are well-suited approaches for studying contemporary research phenomenon that seek to explore "how" and "why" research questions. ${ }^{271}$ Embedded case study design encourages the use of a "multiplicity of evidence" to explore "different salient aspects" of a case. ${ }^{272}$ Embedded case studies are particularly useful for research which seeks to reveal "nested" contextual elements in experiences. For example, in her dissertation examining successful Spanish program instructions in three Midwestern high schools, Theiler used an embedded multiple case study approach to understand how different contextual factors influenced student learning experiences. ${ }^{273}$ The ability to investigate phenomenon of interest at multiple levels, as well as across and between relationships, is one of the hallmarks of the embedded case study design. ${ }^{274}$ An embedded case study design allows me simultaneously to: (a) provide a rich account of a complex interaction space of individual experiences; and (b) situate those experiences within a broader understanding of how historians' information practices and needs relate to their use of digitized photographs.

\footnotetext{
${ }^{271}$ Yin, 9.

${ }^{272}$ Roland Scholz and Olaf Tietjie, "Types of Case Studies," in Embedded Case Study Methods, ed. Roland Scholz and Olaf Tietjie (Thousand Oaks, CA: Sage Publications, 2002), 10.

${ }^{273}$ Janine M. Theiler, "A Shared Story of Successful Spanish Learning: An Embedded Multiple Case Study" (PhD Diss., University of Nebraska Lincoln, 2012).

${ }^{274}$ Yin, 42.
} 
Case studies are well-suited to exploratory research. As Choemprayong and Wildemuth observe, "Case studies are often used in exploratory studies to define phenomena worth studying further." ${ }^{275}$ According to the LIS literature reviewed in section 2.3, there are few qualitative examinations of image use in digital environments. Existing empirical research tends to focus on descriptive or quantitative summaries of image use based on either transaction log analysis (e.g., the number of times an image in a collection has been accessed; analysis of retrieval queries by user) or to assess how well specific metadata elements facilitate image use. A case study design was deemed appropriate to my topic of investigation given the likelihood of complex data collection and mixture of analytic approaches. Case study methodologies also benefit from the formulation and application of theoretical propositions, which played a crucial role in the development of my research inquiry. Theoretical propositions are seen as useful for orienting analytic strategies in case study research. ${ }^{276}$

The methodological foundation for this research comes from phenomenology. ${ }^{277} \mathrm{My}$ research questions were anchored in a phenomenological perspective which stipulates that experiences form the basis for how meaning is constituted. Phenomenological approaches enable researchers to "describe, understand and interpret the meanings of experiences."278 Phenomenology originated from the work of German professor of philosophy Edmund Husserl

\footnotetext{
${ }^{275}$ Songphan Choemprayong and Barbara Wildemuth., "Case Studies," in Applications of Social Research Methods to Questions in Information and Library Science, ed. Barbara Wildemuth (Westport, CT: Libraries Unlimited, 2009), 52.

${ }^{276}$ Yin, 130.

${ }^{277}$ It is important to note that phenomenology can be used both as a method and a methodology. My dissertation research employs the latter approach, seeking to uncover how a phenomenon of interest is experienced through an investigation of "inter-subjectively constructed meanings." See: T.D. Wilson, "Alfred Schutz, Phenomenology and Research Methodology for Information Behaviour Research," New Review of Information Behaviour Research 3 (2002): 71-82.
}

${ }^{278}$ Michael Bloor and Fiona Wood, "Phenomenological Methods," in Keywords in Qualitative Methods (London: Sage Publications, 2006), 129. 
in the late $19^{\text {th }}$ century and early $20^{\text {th }}$ century. Its aim was to provide new methodologies in philosophy for the analysis and description of various types of "pure experiences."279

Phenomenological approaches prioritize understanding the role that perception plays in constituting meaning, a perspective that meshed well with my research focus on visual phenomena. ${ }^{280}$ The phenomenological perspective seeks to understand "how persons construct meaning" through examinations of their experiences with phenomenon of interest. ${ }^{281}$ According to Husserlian phenomenology, perception is a mode of experience which can enact, or bring forth, meaning through human consciousness and intentionality. ${ }^{282}$ Thus, attempts to understand processes of meaning-making should attend to perceptions as experience. Dourish explains that with the phenomenological perspective, "Perception begins with what is experienced, rather than beginning with what is expected; the model is to 'see and understand' rather than 'understand and see." $" 283$

In this research I adopted an interpretivist stance, which proposes that meaning-making is a socially constructed practice, constituted by, and situated within, specific contexts. Interpretivist researchers "attempt to understand phenomena by accessing the meanings that

${ }^{279}$ Husserl justified this orientation thusly: "Since the region of being to be pointed out is none other than that we refer to on essential grounds as "pure experiences," "pure consciousness" with its pure "correlates of consciousness" (=contents or noemata), and on the other side its "pure I," we observe that it is from the I, the consciousness, the experience as given to us from the natural standpoint, that we take our start." See: Barry Smith, Cambridge Companion to Husserl (Cambridge: Cambridge University Press, 1996), 335.

${ }^{280}$ As visual phenomenon, photographs can be interpreted in numerous ways. For a helpful theoretical orientation on the practices of using photographs as research objects, see: John Collier and Malcolm Collier, Visual Anthropology: Photography as a Research Method (Albuquerque: University of New Mexico Press, 1986).

${ }^{281}$ Wilson, 193.

${ }^{282}$ Farber's reading of Husserl suggests that perceptions are possibilities that are given as "empty intentions"; it is when our "interest is awakened", when we have a "consciousness that reaches out" that describes the intentionality of perception. See: Marvin Farber, The Aims of Phenomenology (New York: Harper \& Row, 1966), 114.

${ }^{283}$ Paul Dourish, Where the Action Is: The Foundations of Embodied Interaction (Cambridge, MA: MIT Press, 2001), 21. 
participants assign to these. They are aware that their data gathered are their own constructions of other people's constructions of their perceptions of the world." ${ }^{284}$ An important aspect of interpretivist research involves recognizing the role that the researcher plays in the research process. According to Walsham, interpretive researchers are either "outside observers" identified as researchers who maintain distance from the object(s) of study in the field; or "involved researchers" - meaning participant observers or action researchers who gain insider perspectives by joining the individual(s) or organization(s) being studied. ${ }^{285}$

Interpretivist approaches are particularly beneficial for research aiming to better understand emergent factors in information needs and use. Myers and Avison argue, "Attention would shift from identifying the user's essential, foundational and enduring set of information needs, to identifying how different information availability enabled the juxtaposition of quantity and quality, the shifting back and forth from numbers and calculation to persons and values." 286 Interpretive designs can provide a rich, comprehensive understanding of subjective dimensions impacting information systems use. Building on Habermas' theory of communicative action, Mingers argues that mixed methods approaches to information use help to reveal perspectives on participants' experiences in material, social, and personal worlds. ${ }^{287}$ Ma points out that mixed

\footnotetext{
${ }^{284}$ Marcus Keutel and Werner Mellis, “An In-Depth Interpretive Case Study in Requirements Engineering Research: Experiences and Recommendations," (University of Cologne, 2011), 3.

${ }^{285}$ Geoff Walsham, “Interpretive Case Studies in IS Research: Nature and Method,” European Journal of Information Systems 4 (1995): 77.

${ }^{286}$ Michael Myers and David Avison, "Information System Use as a Hermeneutic Process," in Qualitative Research in Information Systems, ed. Michael Myers and David Avison (London: Sage Publications, 2002), 237.

287 John Mingers, “Combining IS Research Methods: Towards a Pluralist Methodology,” Information Systems Research 12, no.3 (2001): 240-59.
} 
method approaches in studies of information are especially "useful for analyzing various

contexts for information activities." 288

Investigating how scholars experience visual materials can be helpful for revealing the emergent qualities and attributes that make that experience meaningful for participants. McIntyre and Smith explain:

Photographs, symbols, and words in themselves and apart from the meanings and interpretations given them by persons or other creatures possessing mentality, are only so many marks on paper. Their intentionality - their 'representing,' or being 'of' or 'about' things other than themselves -is therefore not a character they have intrinsically, insofar as they are merely the physical objects that they are, but is derivative from their relation to intentional mental states. ${ }^{289}$

Following this argument, visual representations become something through the relationships and meanings we inscribe unto them. Shifting toward analysis of experiences of things, then, can help us understand how things become meaningful. ${ }^{290}$

\subsection{Participants}

The research population for this study included all English-speaking, self-identified historians. ${ }^{291}$ A mix of purposive sampling approaches were used. Purposive sampling is a deliberately non-random method for recruiting individuals. It can provide "information-rich

\footnotetext{
${ }^{288}$ Lai Ma, "Some Philosophical Considerations in Using Mixed Methods in Library and Information Science Research," Journal of the American Society for Information Science 63, no. 9 (2012): 1865.

${ }^{289}$ Ronald McIntyre and David Woodruff Smith, "Theory of Intentionality," in Husserl's Phenomenology: A Textbook, eds. J.N. Mohanty and William R. McKenna (Washington, D.C.: Center for Advanced Research in Phenomenology and University Press of America, 1989), 148-149.

${ }^{290}$ In Logical Investigations, the phenomenologist Edmund Husserl referred to this philosophy as "back to the 'things themselves."'

${ }^{291}$ This is a conscious decision made by the researcher to extend participation beyond a previous pilot study, which limited participation to academic faculty historians at U.S. institutions. Additionally, none of the four archival collections whom I contacted in the early stages of this research were capturing individual scholarly use of digital images.
} 
cases" to enable in-depth study of a particular phenomenon. ${ }^{292}$ Three avenues were used to recruit potential research participants: (1) professional mailing lists, online forums and Twitter; (2) individuals at five local institutions; and (3) individuals recommended to me through colleagues and other recruited participants. I observed the general principles recommended by Corbin and Strauss regarding a grounded theory approach to data saturation. ${ }^{293}$

All recruitment invitations specified that to qualify for inclusion in this study, historians must have previous experience using digitized archival photographs in scholarly activities. Scholarly pursuits could include teaching, publications, presentations, or research projects. Initial recruitment invitations were posted online or emailed to potential participants, depending on the targeting strategy. ${ }^{294}$ Recruitment strategies happened simultaneously, though the third strategy (snowball sampling) continued throughout data collection and analysis. Each strategy is discussed in greater detail below.

1) Professional Mailing Lists, Online Forums, and Twitter Recruitment: A recruitment post was sent to the members of ten electronic mailing lists that are part of the Humanities and Social Sciences Net Online (H-NET), a large online discussion network. Thematic lists focusing on a wide-range of historical sub-disciplines were selected. ${ }^{295}$ The decision to post to professional mailing lists was a conscious effort to broaden the sample population

\footnotetext{
${ }^{292}$ According to Patton, information rich cases "are those from which one can learn a great deal about issues of central importance to the research." See: Michael Quinn Patton, Qualitative Research and Evaluation Methods, $3^{\text {rd }}$ ed. (Thousand Oaks, CA: Sage Publications, 2002), 230.

${ }^{293}$ According to Corbin and Strauss, data saturation in grounded theory occurs when categories are sufficiently welldeveloped to formulate and explain theoretical assumptions. Juliet Corbin and Anselm Strauss, Basics of Qualitative Research: Techniques and Procedures for Developing Grounded Theory, 2nd ed. (Newbury Park, CA: Sage Publications, 2008), 139-140.

${ }^{294}$ Recruitment invitations differed depending on sampling strategy.

295 The following mail lists were selected: H-AFRO AM, H-Amstdy, H-Memory, H-POL, H-Public, H-South, HSurvey, H-US1918-45, H-USA, H-Women.
} 
beyond recruitment of only historians at academic institutions. A recruitment post was also listed on the websites of the Organization of American Historians $(\mathrm{OAH})^{296}$ and the American Studies Association (ASA) Visual Culture Caucus ${ }^{297}$, and distributed through my personal Twitter account. ${ }^{298}$ The recruitment post invited self-identified historians that have used digitized archival photographs in scholarly pursuits to contact me if they were willing to participate in my doctoral research. ${ }^{299}$

2) Identified individuals at local institutions. Potential participants at five area institutions were also invited to participate in this study. ${ }^{300} \mathrm{I}$ created an initial list of historians along with their contact information and thematic research area(s) using faculty web pages at each institution. I then ranked each individual according to the likelihood that they fit the inclusion criteria for this study. For example, historians who specified an interest in digital history were ranked as "1". Once the ranking was complete, I emailed a recruitment invitation to individuals assigned to either "1" or " 2 " categories. ${ }^{301}$

3) Identified individuals at other institutions. I anticipated recruiting at least four individuals through snowball sampling. Over the last year of developing my research proposal, I was given the names and contact information for six historians who actively use digitized

\footnotetext{
${ }^{296}$ During a previous study undertaken by the researcher, a description of the study and a recruitment invitation was posted on the OAH website http://www.oah.org/.

${ }^{297} \mathrm{http}: / /$ www.theasa.net/caucus_visual/

${ }^{298}$ http://www.twitter.com/amchass

${ }^{299}$ See Appendix A for Interview Recruitment Post.

${ }^{300}$ The five institutions were: The University of North Carolina at Chapel Hill, The University of North Carolina at Greensboro, Duke University, North Carolina Central University, and North Carolina State University. These institutions were chosen because they are all offer graduate-level degrees in history and are located within a sixtymile driving radius of the researcher.

${ }^{301}$ See Appendix B for Interview Recruitment Email.
} 
photographs. I also expected to gather additional names and contacts through

recommendations from other participants.

\begin{tabular}{|c|c|c|}
\hline TARGETING STRATEGY & SAMPLING TECHNIQUE & EXPECTED PARTICIPANTS \\
\hline Mailing lists, online forums, Twitter & Criterion; homogenous & 10 \\
\hline $\begin{array}{c}\text { Identified individuals at local } \\
\text { institutions }\end{array}$ & $\begin{array}{c}\text { Convenience; criterion; } \\
\text { snowball }\end{array}$ & 5 \\
\hline $\begin{array}{c}\text { Identified individuals at other } \\
\text { institutions }\end{array}$ & Snowball; criterion & 5 \\
\hline
\end{tabular}

Table 1. Recruitment strategies

\subsection{Data Collection}

Once individuals agreed to participate, a fact sheet to obtain consent was sent and an interview time was arranged. ${ }^{302}$ For each participant, I created both a manila folder and a computer folder with each participant's name. I also created a spreadsheet with participant IDs and basic demographic information. As part of their recruitment, participants were instructed to pre-select two digitized photographs they have previously used in instructional teaching and research endeavors. I advised participants that their chosen photographs would be used as prompts for eliciting descriptions about their specific experiences related to image use. The decision to have participants select images they had already used was made in accordance with the theoretical assumptions of this study, which stated that information use is always situated and contextual. Thus, in order to understand their experiences, it followed that I should use specific examples of use to explore how and why they have used images in their scholarly activities.

Participants were instructed to email me their pre-selected photographs (preferably in URL form, though attachments were accepted) no later than one week before the scheduled interview. I also asked them to provide supplementary materials related to their use of images

\footnotetext{
302 See Appendix C for Fact Sheet.
} 
(e.g., publications in which they have used digitized photographs, syllabus in which they have instructed students on evaluating visual materials). To ensure that participants qualified for study inclusion, I reviewed each of their selections within twenty-four hours of receipt. Three of my participants had to rescind participation after it was discovered that they had only used illustrations or archival photographs in print. Though I had initially conceived of creating customized web pages called Photograph Scenarios for each participant containing links to their pre-selected photographs, nearly all of my participants opted to discuss their selected photographs via their original online location. While this approach worked adequately for this study, it must be noted that URL locations for items can frequently change and often do not provide permanent links.

Interview sessions took place with recruited participants at a setting of their choice, provided it was equipped with internet access (see Table 2). The majority of interviews were conducted through Skype. Most sessions lasted between 1 and 1.5 hours and were digitally recorded using Amalto Call Recorder for Skype. Interviews were then transcribed verbatim. At the beginning of each session, I introduced myself, the purpose of the study, and verified participant consent. ${ }^{303}$ In the first part of the interview, participants were asked to answer demographic information and answer questions about their image use practices. ${ }^{304}$ In the second part of the interview, participants were instructed to open a web browser to the location of their pre-selected photographs. I used techniques from photo elicitation interviewing (PEI) ${ }^{305}$ and

\footnotetext{
${ }^{303}$ See Appendix D for Verbal Consent Script.

${ }^{304}$ See Appendix E for Semi-Structured Interview Protocol.

${ }^{305}$ PEI originated from the work of anthropologist John Collier in his 1957 study on the acculturation process of a migrant population in England. See: John Collier Jr., "Photography in Anthropology: A Report on Two Experiments," American Anthropologist 59 (1957): 843-59.
} 
concurrent verbal probes ${ }^{306}$ to elicit descriptions from participants about their experiences using digitized photographs as forms of evidence. The PEI portion of the protocol contained 15 prescripted interview questions along with several verbal probes. ${ }^{307}$

\begin{tabular}{|c|c|c|c|}
\hline PARTICIPANT & PRIMARY RESEARCH AREA(S) & SAMPLE COURSE & DEGREE \\
\hline Andrew Nelson & $20^{\text {th }}$-century African American history & $\begin{array}{l}\text { "Intro to American } \\
\text { History" }\end{array}$ & $\begin{array}{l}\text { PhD, American } \\
\text { Studies }\end{array}$ \\
\hline Daegan Miller & $\begin{array}{l}19^{\text {th }} \text {-century American alternative } \\
\text { green culture }\end{array}$ & $\begin{array}{l}\text { "The Ecology of Vision: } \\
\text { A History of Looking at } \\
\text { the American } \\
\text { Landscape" }\end{array}$ & PhD, History \\
\hline Edward Roach & Technology and the Gilded Age & $\mathrm{N} / \mathrm{A}$ & MA, History \\
\hline Edward Salo & $\begin{array}{l}\text { Historical preservation and U.S., } \\
1900-1945\end{array}$ & $\begin{array}{l}\text { "Intro to Historic } \\
\text { Preservation" }\end{array}$ & $\begin{array}{l}\text { PhD, Public } \\
\text { History }\end{array}$ \\
\hline Zachary Hilpert & Urban history & "Focused Inquiry 1" & $\begin{array}{l}\mathrm{PhD} \text {, American } \\
\text { Studies }\end{array}$ \\
\hline Jeannette Gabriel & African-Americans and labor history & $\mathrm{N} / \mathrm{A}$ & MA, History \\
\hline Jennifer Black & $19^{\text {th }}$-century American visual culture & $\begin{array}{c}\text { "University Writing } \\
\text { Seminar: Images of } \\
\text { America" }\end{array}$ & $\begin{array}{l}\text { PhD, American } \\
\text { History and } \\
\text { Visual Studies }\end{array}$ \\
\hline Jerome Krase & Urban neighborhoods & $\begin{array}{l}\text { "The Peopling of New } \\
\text { York: A Visual Virtual } \\
\text { Approach" }\end{array}$ & PhD, Sociology \\
\hline Jonathan Grunert & Science and technology studies & "US History Survey 1" & MA, History \\
\hline $\begin{array}{l}\text { Kate Sampsell- } \\
\text { Willmann }\end{array}$ & $20^{\text {th }}$-century intellectual history & "US History since $1865 "$ & PhD, History \\
\hline Kristen Turner & Opera, gend & "Women and Music" & $\mathrm{PhD}$, Music \\
\hline Lisa Jackson & Early $20^{\text {th }}$-century gender & $\mathrm{N} / \mathrm{A}$ & MA, History \\
\hline $\begin{array}{c}\text { Patricia } \\
\text { McCormack }\end{array}$ & Aboriginal studies & $\begin{array}{l}\text { "Historical Perspectives } \\
\text { in Native Studies" }\end{array}$ & $\begin{array}{c}\mathrm{PhD}, \\
\text { Anthropology }\end{array}$ \\
\hline Stephen Gold & International migration & $\begin{array}{l}\text { "Race, Politics, and } \\
\text { Social Structure" }\end{array}$ & $\mathrm{PhD}$, Sociology \\
\hline Wendy Clark & $\begin{array}{l}19^{\text {th }} \text {-century environmental History in } \\
\text { the South }\end{array}$ & $\begin{array}{l}\text { "US History from 1877- } \\
\text { present" }\end{array}$ & PhD, History \\
\hline
\end{tabular}

Table 2. Participants by primary research area, sample teaching course, and degree

I followed similar protocol to approaches outlined in the PEI literature, which advocates for the use of photographs (or other visual prompts) to guide the interview process. In PEI, "the

\footnotetext{
${ }^{306}$ Verbal probing is an interviewing technique which offers a "focused and productive approach" to eliciting cognitive responses. See: Gordon Willis, Cognitive Interviewing: A Tool for Improving Survey Design (Thousand Oaks, CA: Sage Publications, 2005), 50. Verbal probing was initially developed and used in survey methodology assessment, as a supplement to Think Aloud Protocol (TAP).

${ }^{307}$ See Appendix F for Photo-Elicitation Interview Protocol.
} 
researcher assumes that the images, the meaning(s) we attribute to them, the emotions they arouse in the observer, and the information they elicit generate insights that do not necessarily or exclusively correspond to those obtained in verbal inquiry." 308 Concurrent verbal probing, a technique outlined largely in the context of Willis' Cognitive Interviewing procedures, was used to augment participant discourse where appropriate. According to Willis, concurrent verbal probing as a technique involves: "a) the interviewer asking the question, b) the subject answering the question, c) the interviewer asking a probe question, d) the subject answering the probe question, and e) possibly, further cycles of (c-d)." ${ }^{309}$ Different types of verbal probes (e.g., "interpretation/comprehension probes") can be used to elicit further description on topics of interest. While the think-aloud protocol (TAP) is often used as a technique for explicating cognitive information processes, the classical model put forth by Herbert Simon and Allen Newell recommends avoidance of probing participants during the protocol. Since visual materials are intrinsically mediated forms, I anticipated that it might be difficult to generate meaningful discussion without the use of probing. ${ }^{310}$ Therefore, concurrent verbal probing was considered a more appropriate technique than TAP to use in PEI.

At the close of each interview session, I thanked participants for their time and encouraged them to contact me with any questions. I also asked about their willingness to be

\footnotetext{
${ }^{308}$ Elisa Bignante, "The Use of Photo-Elicitation in Field Research," EchoGeo 11 (2010): 2.

${ }^{309}$ Gordon Willis, “Cognitive Interviewing: A 'How to' Guide,” 1999 Meeting of the American Statistical Association (Research Triangle Park, NC: Research Triangle Institute, 1999), 7.

${ }^{310}$ In Samuel Wineburg's classic study of historians' problem-solving, historians were asked to think aloud while assessing three pictures depicting the Battle of Lexington. He writes, "For historians, the picture evaluation task was an exercise in exploring the limits of historical knowledge. The end result was more a suggestion than an answer, more a forced choice from flawed alternatives than a committed decision executed with resolve." Samuel Wineburg, "Historical Problem Solving: A Study of the Cognitive Processes Used in the Evaluation of Documentary and Pictorial Evidence," Journal of Educational Psychology 83, no.1 (1991):76. Since the aim of my research is to better understand how historians evaluate and assess digitized materials, the use of verbal probes will be an essential aspect in generating participant discourse around materials.
} 
contacted for any follow ups, in case further clarification was needed. Following each interview, I collected any supplementary documents they could provide related to examples of their image use (e.g., syllabi, class materials, publications, presentations, etc.).

\subsection{Data Management}

At the onset of my research, I developed an organizational approach to data management planning for two main organizational aspects: data storage and data organization. Both strategies were equally important to the research process. Keeping my documentation wellorganized and safely stored would also help ensure that it remained accessible.

Data storage referred to how I planned on keeping data stored locally. I decided to primarily organize my materials using a hierarchal folder structure on my windows laptop. I created a folder called dissertation that had three sub-folders: analysis, data collection, and proposal. I used the analysis folder to store all of my memos related to coding decisions, along with a master spreadsheet of thematic categories used to code my data. The data collection folder contained a master recruitment spreadsheet and individual folders for each study participant. The proposal folder held all of the documents related to my dissertation proposal including the two interview protocols used and recruitment materials used in the study. It also contained administrative-related documents, such as the drafts of my Institutional Review Board (IRB) documents.

Data organization focused on how I could best organize and provide access to the sources used as evidence in my dissertation. My case study design suggested to me that I could treat each participant's collected materials as a single case. Within the data collection folder structure, I created a single folder for each participant which contained an interview transcript (word document), the original file recorded from the Skype interview call (.mp3 format), and the 
de-identified audio file used by the transcriptionist (.mp3 format). I also had to manage secondary source materials, such as the studies I used in the literature review portion of my dissertation. I initially deposited all documents (primarily pdfs) in a single folder on my hard drive called readings. I used Mendeley, a citation management software, to organize and annotate materials.

The second type of source material I had to manage were references about the sources I was referencing in my dissertation. For example, the thematic codes I was going to be assigning to transcripts had to be linked to a codebook with definitions and examples for use in inter-coder reliability tests. I also had to prepare to manage tags used to identify themes from the secondary source literature I collected and annotated. I was also able to use Mendeley tags to identify potential themes of interest, derived from my literature review. An article on historians' information-seeking in digital environments might have three tags: "information-seeking", "digital environments", and "historians." Tagging was useful because it enabled me to find additional themes that I might have missed in my primarily data-driven coding approach.

\subsection{Data Analysis}

In case studies, data from multiple sources are typically integrated in the analytic stage of research. As Baxter and Jack explain: “Each data source is one piece of the 'puzzle,' with each piece contributing to the researcher's understanding of the whole phenomenon. This convergence adds strength to the findings as the various strands of data are braided together to promote a greater understanding of the case." ${ }^{\prime 11}$ The case study researcher must strive to analyze each case as a whole, rather than analyzing just the individual pieces of the case.

\footnotetext{
${ }^{311}$ Pamela Baxter and Susan Jack, "Qualitative Case Study Methodology: Study Design and Implementation for Novice Researchers," The Qualitative Report 13, no. 4 (2008): 554.
} 
Stake defines a case as being a "specific, complex, functioning thing." 312 In embedded case studies, more than one unit of analysis can be used to explore a research phenomenon at multiple levels and perspectives. For the purposes of this research, two units of analysis were used. The main unit of analysis was identified as: "a historian's experiences using digitized photographs as evidence." An embedded unit of analysis, or subunit, was defined as "a historian's specific use of a digitized photograph as evidence in research or instruction." Embedded case studies present particular challenges for novice researchers. Yin points out that one of the major pitfalls of embedded case study design is a failure to return to the main unit of analysis. ${ }^{313}$ Therefore, it was important that I adopt analytic procedures that enabled the analysis of materials both within cases and across cases.

1) Collecting different types of data. Four types of data were collected for each case: transcripts from the semi-structured interviews; photographs from the photo-elicitation portion of the interview; transcripts from the photo-elicitation portion of the interview; and supplementary documents (e.g., syllabi, class materials, publications, presentations, etc.) provided by participants showing how they have used images.

2) Choosing initial method for analysis. Thematic analysis was chosen as an appropriate analytic method to analyze both textual and visual materials. Thematic analysis can be defined as "a method of identifying, analyzing and reporting patterns (themes) within data." ${ }^{314}$ It is often considered a technique of content analysis. In this study, a hybrid

\footnotetext{
${ }^{312}$ Robert E. Stake, The Art of Case Study Research (Thousand Oaks, California: Sage Publications, 1995), 2.

${ }^{313}$ Robert Yin, Case Study Research: Design and Methods $2^{\text {nd }}$ Edition (Thousand Oaks, CA: Sage Publishing, 1994), 44.

${ }^{314}$ Virginia Braun and Victoria Clarke, "Using Thematic Analysis in Psychology," Qualitative Research in Psychology 3, no.2 (2006): 79.
} 
approach to thematic analysis was adopted, following the example of Fereday and Eimear Muir-Cochrane. ${ }^{315}$

3) Developing the code manual featuring categories and a coding scheme. The coding manual was developed iteratively through a mixed deductive-inductive approach. Each code entry contained a code label, definition of the theme, and description of how to know when the theme occurs. In the initial, pre-defined stage of analysis, I created a broad list of a priori categories derived from the literature review, my research questions, and the theoretical propositions of the study. Examples of potential broad categories I used included: "materiality", “experiences”, “evidence.” I then read through interview transcripts for each case, highlighting descriptions related to these categories. In the emergent stage of data analysis, I did a second-read through of transcripts to look for "important moments." 316 While there are numerous categories that could be anticipated, the goal of this portion was to allow for patterns and themes to emerge from the data. The constant comparative method was used to generate new categories as they emerged, and then to combine/revise previous categories. ${ }^{317}$ I also used memos to document decisions made about categorization. According to Zhang and Wildemuth, "To improve the credibility of qualitative content analysis, researchers not only need to design data

\footnotetext{
315 Jennifer Fereday and Eimear Muir-Cochrane, "Demonstrating Rigor Using Thematic Analysis: A Hybrid Approach of Inductive and Deductive Coding and Theme Development," International Journal of Qualitative Methods 5, no.1 (2006): 1-11. Their approach integrates inductive-deductive coding with theme development, allowing codes to emerge from the data while also supplying theoretical propositions to bind analysis for each case.

${ }^{316}$ According to Fereday and Eimear Muir-Cochrane, the process of inductive coding involves "recognizing (seeing) an important moment and encoding it (seeing it as something) prior to a process of interpretation." See: Fereday and Eimear Muir-Cochrane, 4.

${ }^{317}$ The constant comparative method is a grounded theory technique for developing inductive coding categories. See: Barry Glaser and Anselm Strauss, The Discovery of Grounded Theory: Strategies for Qualitative Research (New York: Aldine, 1967).
} 
collection strategies that are able to adequately solicit the representations, but also to design transparent processes for coding and drawing conclusions from the raw data." ${ }^{318}$

4) Testing the reliability of codes. After an initial coding scheme was developed, I recruited a colleague to help validate my coding scheme. Two interview transcripts were provided to the colleague, along with the coding manual. We reviewed the coding manual and discussed definitions, examples, and classification rules. My colleague then proceeded to code both transcripts. Upon completion, I compared versions of our coded transcripts, noting any discrepancies between coding which were then clarified in the manual. A final coding scheme was developed following modifications.

5) Integrating data sources within each case. In case study research, sources of data are typically integrated or "mixed together" during the data analysis procedure. In this research, sources of data in a variety of formats and contexts were used. As an example, I needed to integrate the semi-structured interview portion with the PEI portion of the interview for interpretation and analysis. Additionally, documents related to participant's prior image use (e.g., syllabus, teaching presentations) also needed to be integrated into each case. The goal was to assemble all participant materials and begin to formulate and approach the collection as the building of each case.

6) Summarizing data and identifying additional themes. In this step, key points for each case were outlined through a review of the transcripts, document, and memos. Each summary (see section 4.1) reflects my initial processing of the information for each case, providing "the opportunity to sense and take note of potential themes in the raw data."319

\footnotetext{
${ }^{318}$ Zhang and Wildemuth, 313.

${ }^{319}$ Fereday and Muir-Cochrane, 7.
} 
7) Conceptually clustering themes into categories. I then used the coded themes to "make sense" of the data across the different themes, with an eye towards Patton's recommendation of developing categories that achieve both internal homogeneity and external heterogeneity. ${ }^{320}$ What kinds of patterns emerged in each case? How did they inform my understanding of the larger research phenomenon? In this stage of the process, "discrete bits of information come together to make a more economical whole that, analytically speaking, is more than the sum of its parts." ${ }^{321}$

8) Synthesizing descriptive themes across cases. Following the work of Thomas and Harden, I used thematic synthesis as a strategy to generate analytical themes that integrated findings across each case. ${ }^{322}$ This enabled me to return back to the main unit of analysis in this study, historians' overall experiences using digitized photographs as evidence. Through an iterative process of analysis and interpretation of descriptive themes, I identified six analytical themes that characterized historians' experiences. Cycling through this process enabled me to revise analytical themes until they were "sufficiently abstract." 323 Tying together historians' examples of use with the descriptive themes and categories that surfaced from my thematic analysis helped to triangulate my interpretation and analysis (see Figure 2).

\footnotetext{
${ }^{320}$ Patton defines internal homogeneity as "the extent to which the data that belong in a certain category hold together or 'dovetail' in a meaningful way" while external homogeneity is "the extent to which differences among categories are bold and clear." See: Patton, Qualitative Research and Evaluation Methods, 465.

${ }^{321}$ Matthew Miles, A. Michael Huberman, and Johnny Saldana, Qualitative Data Analysis: A Methods Sourcebook, 3rd Ed. (Thousand Oaks, CA: Sage Publications, 2013), 290.

${ }^{322}$ James Thomas and Angela Harden, "Methods for the Thematic Synthesis of Qualitative Research in Systematic Reviews." BMC Medical Research Methodology 8, no.45 (2008).

${ }^{323}$ Ibid, 7.
} 


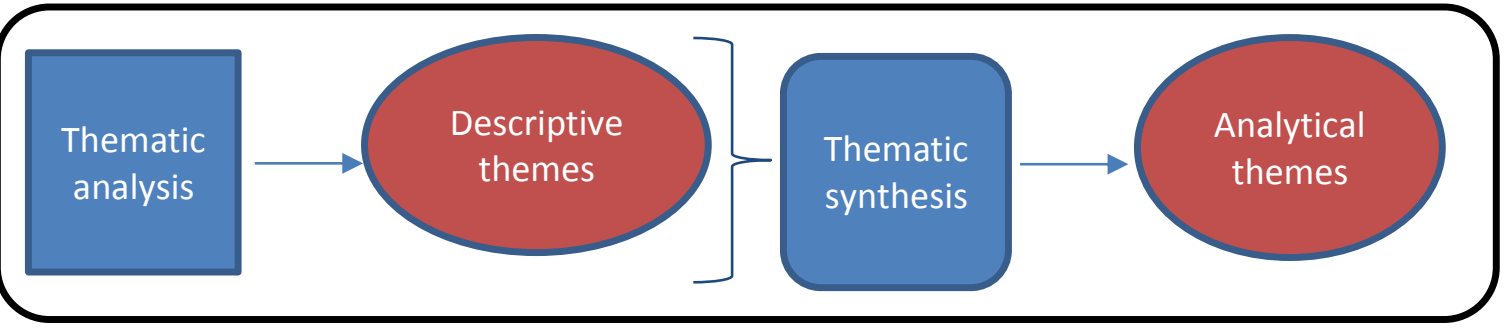

Figure 2. Stages of analysis and interpretation

9) Distinguishing between potential use and evidential use. At this stage in my analysis and interpretation, I began to consider how my six analytical themes functioned conceptually within the broader scope of information use behavior. In particular, I noted how my analytical themes took place during two spheres, what I'm calling potential image use and evidential image use. The activities, practices, and processes that take place within both spheres are not necessarily linear or sequential and can occur multiple times throughout image selection and image use. Distinguishing between these two spheres helped me to capture the dimensions that spurred actual use of materials, while also exploring factors that motivated historians in potential image use.

10) Reporting on findings through cases. Findings were then anchored in the data through case reporting. I aimed to strike a balance between thickly describing study procedures and findings, and making interpretive connections from the findings. The literature reviewed in Chapter 2 also provided useful context for situating interpretation. According to Patton, in reporting findings researchers should provide "sufficient description to allow the reader to understand the basis for an interpretation, and sufficient interpretation to allow the reader to understand the description." 324

${ }^{324}$ Patton, 503-504. 


\subsection{Reliability and Validity}

Establishing trustworthiness is a common concern for researchers seeking to ensure the reliability and validity of their interpretive research. Lincoln and Guba identify four types of criteria which can characterize trustworthiness in interpretive research: transferability, dependability, confirmability, and credibility. ${ }^{325}$ I will briefly discuss each of these criteria, along with how I ensured compliance in my research methodology.

Transferability refers to the degree to which research findings can be generalized and made applicable across a variety of contexts - what the authors term as "fittingness." 326 Lincoln and Guba suggest that the researcher's use of thick description is one technique for achieving transferability; by capturing rich details about the research setting, sample, and data collection procedures, the researcher provides information regarding the transferability of findings to other contexts. I made every effort to be transparent by providing detailed discussions of research procedures, and used memos as a strategy for documenting decision-making throughout quantitative and qualitative phases of data collection and analysis.

Dependability describes the extent to which the research design is repeatable (though not necessarily expected to generate the same findings) as a "prototype model." 327 One means for addressing dependability is the use of multiple methods, which can increase both the credibility and dependability of the study. ${ }^{328}$ This case study triangulated multiple sources of data (e.g., interview transcripts, participant-selected digitized photographs, documents related to prior

\footnotetext{
${ }^{325}$ Yvonne Lincoln and Egon Guba, Naturalistic Inquiry (Beverly Hills, CA: Sage Publications, 1985), 289-331. ${ }^{326}$ Ibid, 124.

${ }^{327}$ Andrew K. Shenton, "Strategies for Ensuring Trustworthiness in Qualitative Research Projects," Education for Information 22 (2004), 71.

${ }^{328}$ Egon Guba, "Criteria for Assessing the Trustworthiness of Naturalistic Inquiries," Educational Communication and Technology 29, no.2 (1981): 75-91.
} 
image use), multiple data collection techniques (verbal probing, photo-elicitation, semistructured interviews), and multiple stages of interpretation and analysis (thematic analysis, thematic synthesis). In particular, the use of PEI offered a complementary approach to semistructured interviews. ${ }^{329}$

The non-directive nature of both PEI and semi-structured interviewing suggested that careful formulation and pilot testing of the protocol would be essential to ensure robust participation from my participants. PEI research also presents ample possibilities for derailment into stream-of-consciousness interview accounts. According to Bignante, PEI researchers "must constantly strive to strike the right balance between the desire to let informants express themselves freely, being open to unexpectedly interesting insights that arise during the course of the research, and rigor in ensuring research objectives are addressed and discussion is re-directed to the subject of inquiry." ${ }^{330}$ In accordance with the stated goals of the study, a conscious effort was made during each interview to direct attention towards participant descriptions of their experiences using photographs. Another technique to aid in the establishment of dependability is the use of an audit trail to document research procedures. As mentioned previously, I used a thick description approach to documenting decisions and relevant processes throughout data collection and analysis.

Confirmability refers to the extent that research findings reflect respondents rather than researcher bias. What were the researcher's design decisions and how might that have impacted collection and analysis? How did the researcher develop coding schemes used in analysis? The use of memos throughout data collection and analysis served to document my decisions

\footnotetext{
${ }^{329}$ Francesco Lapenta, "The Image as a Form of Sociological Data: A Methodological Approach to the Analysis of Photo-Elicited Interviews," (PhD diss., University of London, 2004), 74.

${ }^{330}$ Bignante, 11.
} 
regarding design, sample, collection, and analysis. ${ }^{331}$ Memos reflected my commitment to reflexive analysis, which encouraged continual reflection on roles and perceived biases throughout the course of my research procedures. ${ }^{332}$ An important initial step in my research process involved the explication of my philosophical and epistemological groundings for this research (see section 3.2 for elaboration), acknowledging the role that potential bias has in research design and procedure.

In terms of methodology, reflexive approaches in interpretive research favor "methods in which informants took a greater part in contributing to the uncovering and creation of meaning." 333 My research design was informed by an interpretive, phenomenological perspective, aimed at drawing out participant's descriptions of their situated, contextual experiences using digital photographs - a participant-generated thick description. One aim of this research was to "make visible" 334 the process of meaning-making for historians - itself a reflexive goal. ${ }^{335}$

It is important to acknowledge how bias has been introduced into this research design and study. The semi-structured interviews used participant-selected photograph scenarios as prompts for eliciting descriptions. This suggests potential bias on at least three levels. First, using photographs for the primary purpose of eliciting descriptions may have introduced a level of

\footnotetext{
${ }^{331}$ Guba, 87.

${ }^{332}$ According to Finlay: "Reflexive analysis in research encompasses continual evaluation of subjective responses, intersubjective dynamics and the research process itself. It involves a shift in our understanding of data collection from something objective that is accomplished through detailed scrutiny of 'what I know and how I know it' to recognizing how we actively construct our knowledge." See: Linda Finlay, “'Outing' the Researcher: The Provenance, Process, and Practice of Reflexivity," Qualitative Health Research 12, no.4 (2002).

${ }^{333}$ Marcus Banks, “Analyzing Images” in The SAGE Handbook of Qualitative Data Analysis, ed. Uwe Flick (London: SAGE Publications, 2014), 401.

${ }^{334}$ Banks suggests that visual methods can "make visible... something that could not be seen before." Ibid, 403-404.
} 
artificiality into the research setting. Second, participants were asked to comment extensively on decisions and thought processes that have already been made (i.e. their decision to use a photograph in teaching or research). Third, the order in which the photographs were viewed could have potentially influenced participant interpretation. Similar to photographic essays, the resulting experience for participants "may privilege the phenomenological mode, conveying an experience from the interviewees' and researcher's perspectives, or privilege the narrative mode by telling a story from the interviewees' perspectives." ${ }^{336}$ In order to address these potential biases, I organized and integrated explicit discussion and analysis of the photographs used by participants into the case study, including visual representations where possible.

Credibility describes the believability or "truth" of the researcher's findings. According to Suter, "Maximum confidence in the believability of conclusions comes from support provided by participants' agreement, analysis of multiple sources of data, others' interpretations, and prediction based on relevant theoretical models (i.e., a predicted pattern matches an actual pattern). ${ }^{\prime 337}$ How is credibility established? One method is for researchers to orient themselves towards their research population through prolonged engagement. ${ }^{338}$ With regards to my research population, I am confident that my engagement over the last four years through a pilot study, informal conversations, and extensive literature reviews gave me insight and understanding into my population of interest. As an evolving area of research, the literature studying the use of digital resources is limited. However, I have been committed to remaining

\footnotetext{
${ }^{336}$ Marcus Banks, "Presenting Research Results" in Visual Methods in Social Research, ed. Marcus Banks (London: SAGE Publications, 2001), 146.

${ }^{337}$ Suter, Educational Research, 363.

${ }^{338}$ Guba, 84-85.
} 
abreast of new developments in the field and integrating new (or newly discovered) relevant studies into my literature review over the course of this research.

Another strategy for establishing credibility is to provide illustrated quotations from participants, alongside interpretive themes and categories. As a form of interpretive rigor, this strategy can show how "overarching themes are supported by excerpts from the raw data to ensure that data interpretation remains directly linked to the words of the participants. ${ }^{" 339}$ In my analysis and reporting, I looked for "thick description" quotations from participant interviews. In particular, my interpretation and discussion of participants' examples of evidential use relied extensively on extracting direct quotations from supplementary materials (e.g., their dissertation, course power-point presentations, course syllabi) provided to me by participants.

A third strategy is triangulation, or "a process of using multiple perception to clarify meaning, verifying the repeatability of an observation or interpretation. ${ }^{" 340}$ In this research, two types of triangulation helped to establish the credibility of my findings. I used data triangulation, which can be described as the combining of data from different sources. I collected and used a wide variety of data sources, including: interview transcripts, documents related to participants' research and instructional output, and archival photographs, among other items. I also used methodological triangulation, which describes the combination of data from different methods. For example, my interview data was generated from a combined approach of semi-structured interviewing and photo-elicitation interviewing with verbal probing. I also used thematic analysis and thematic synthesis in my analysis and interpretation stages.

\footnotetext{
${ }^{339}$ Fereday and Muir-Cochrane, 3.

${ }^{340}$ Robert E. Stake, “Qualitative Case Studies," in The SAGE Handbook of Qualitative Research, ed. Norman Denzin and Yvonna Lincoln (Thousand Oaks, CA: Sage Publications, 2000), 443.
} 


\section{CHAPTER 4: FINDINGS AND DISCUSSION}

In this chapter, I introduce the findings from my research and situate them within a larger discussion of information use. First, I give a brief biographical summary of my fifteen participants. Next, I describe themes identified during thematic analysis and synthesis. I also discuss two separate spheres where different themes of experience take place, potential image use and evidential image use. Finally, I introduce a conceptual framework that can be used for studying how and why historians use digitized archival photographs as forms of evidence.

\subsection{Summary of Cases}

Below is a brief summary of the fifteen participants included in this study by name, title, department, and affiliation at the time of the study (see Table 3).

\begin{tabular}{|c|c|c|c|}
\hline NAME & TITLE & DEPARTMENT & AFFILIATION \\
\hline Andrew Nelson & Doctoral student & American Studies & University of Maryland at College Park \\
\hline Daegan Miller & Postdoctoral fellow & Center for Humanities & University of Wisconsin-Madison \\
\hline Edward Roach & Public historian & N/A & $\begin{array}{c}\text { Dayton Aviation Heritage National } \\
\text { Historical Park }\end{array}$ \\
\hline Edward Salo & Assistant professor & History & Arkansas State University \\
\hline Zachary Hilpert & Faculty instructor & Focused Inquiry & Virginia Commonwealth University \\
\hline Jeannette Gabriel & Doctoral student & Education & University of Iowa \\
\hline Jennifer Black & Assistant professor & History & Brooklyn College \\
\hline Jerome Krase & Professor emeritus & Sociology & Oirginia Tech \\
\hline $\begin{array}{c}\text { Jonathan Grunert } \\
\text { Kate Sampsell- } \\
\text { Willmann }\end{array}$ & Adjunct professor & Science and \\
\hline Kristen Turner & Doctoral student & History & Music \\
\hline Lisa Jackson & Master's student & History & University of North Carolina at Chapel \\
\hline Patricia & Professor emerita & Native Studies & San Francisco State University \\
\hline McCormack & & Sociology & University of Alberta \\
\hline Stephen Gold & Professor & History & Michigan State University \\
\hline Wendy Clark & Doctoral student & University of Memphis \\
\hline
\end{tabular}

Table 3. Participants by title, department, and institutional affiliation 


\section{Andrew Nelson}

At the time of our interview, Andrew Nelson was a PhD Candidate at the University of Maryland in American Studies. Nelson's dissertation is entitled, "With sight and sound: Black photographic and musical expression in rural Alabama, 1900-1930.” His project uses a collection of eight hundred photographs of African-American individuals in Fayette County Alabama. He decided on this project topic after encountering what he terms "a body of images" in the early $20^{\text {th }}$ century in which people were posing for photographs with musical instruments. He used digitized archival photographs primarily to flesh out this phenomenon throughout the United States.

According to his dissertation abstract, Nelson's study explores "how pictures and songs were used in concert to reiterate particular themes and convey pointed counterhegemonic and race-conscious messages." He used two initial criteria in his image searching: (1) pictures of musicians; and (2) certain types of poses. Google image search provided him with the ability to quickly hone his image searching as well as to connect him with independent scholars with blogs. For example, he mentioned tracking down a photograph that had originally appeared on the cover of a folk album that he had previously seen in a book about West Virginia fiddle music. He was then able to "circumvent the traditional archive" by obtaining a digital scan of the photograph from the author directly.

\section{Daegan Miller}

Dr. Daegan T. Miller received his PhD in History from Cornell University in 2013. At the time of our interview, Miller was completing a postdoctoral fellowship at the Center for Humanities and Institute for Research in the Humanities at the University of Wisconsin Madison. His dissertation explores the construction of alternative landscapes in the $19^{\text {th }}$-century U.S. He 
uses photographs to show how landscapes were "made or envisioned." His interest in using photographs as evidence can be traced back to encountering the work of $19^{\text {th }}$-century landscape photographer AJ Russell. He explains, "They just sort of spoke to me, they grabbed me instantly. And I came across them first in print, but then online. And I was just sort of...totally enthralled by them."

In his dissertation research, he primarily used the archival collections from the New York Public Library, the American Antiquarian Society, and Yale's Beinecke Library. Prior to visiting each archive in person, he consulted the online collections to locate images of interest. He then emailed archivists to request access to images in person. In general, he finds Google image searching and the Library of Congress site to be very helpful for image searching. In his teaching, he aims to help students become more fluent in visual literacy through the use of images as primary sources. For example, he might instruct students to make an argument about the brutality of the Civil War using photographs.

\section{Edward Roach}

Edward Roach works as a historian at the Dayton Aviation Heritage National Historical Park. He received his master's degree in public history. Roach recently completed a book entitled The Wright Company: From Invention to Industry which examines the early years of the Wright Brothers' airplane company. Nearly all of the photographs in the book originated from digitized photographs, drawing largely on collections at the Library of Congress and Wright State University. While Roach does not teach in an academic setting, he instructs a number of interpretive programs for visiting guests to the Historical Park.

He has used digitized photographs (including captions) from these collections in a number of ways, including to: provide historical verification; identify individuals in the 
company; and date other photographs based on similar physical characteristics. He finds the content of the image itself to be the most useful. Some of the other factors that matter to him focus on the provenance of the photograph: knowing where it came from, when it was created, what the archive knows about it, and who to contact at the archive. In particular, Roach praises the ability to zoom in on high-quality digitized photographs to ascertain historical details, explaining that "you can do a lot more with them once you have the scan then you can with that tiny tiny little print."

\section{Edward Salo}

Edward Salo is an Assistant Professor in History at Arkansas State University. He studies historical preservation and the US 1900-1945. Prior to joining as a faculty member, he was a consulting historian for a cultural resources management firm. In that capacity, he used cultural heritage sites like the Florida Memory State Library and Archives collection as reference for ascertaining historical context. He will use Google or Wikipedia in the early stages of research projects, but says "I know I'm not going to hang my hat on that."

His dissertation explores how ferry transportation impacted development in the South Carolina low country. After being unable to find adequate source material for South Carolina, he began looking at photographs and drawings from other nearby states to understand how technology at ferry sites evolved. For example, he found a 1902 photograph of a rope ferry operating in Ocklawaha, Florida by searching online at the Library of Congress Prints and Photographs catalog. He was able to use that image in his dissertation to say "this is what a rope ferry looked like." Salo also seems to recognize the inherent complexities in using digitized photographs as historical evidence. On the one hand, he uses digitized photographs as referential 
sources for reconstructing "how something was." At the same time, he recognizes that he can also use photographs to show what the creator of the image thought was important at that time.

\section{Zachary Hilpert}

Dr. Zachary Hilpert is a faculty member in the Department of Focused Inquiry, University College at Virginia Commonwealth University. He received his $\mathrm{PhD}$ in American Studies, focusing on American history, digital culture, and urban studies. His dissertation examined images of disasters in American cities, spanning the beginning of the Civil War in 1861 through the San Francisco earthquake and fires in 1906. He makes the argument that image makers helped to shape (or reshape) the story of a disaster to both fit and mold the perceptions of the event at the time.

In his research endeavors, he came across "history books" - or assemblages of primarily visual materials from certain events gathered together without a linear narrative. He explains, "So you'd see photographs of the destruction, drawings of people kind of in the midst of the disaster, and then text kind of coupled with those images of varying degrees of actual relationship to the images." These collections for Hilpert helped demonstrate how certain populations became exoticized as "others" in relation to the disaster at hand. For example, after the Johnstown Flood in January 1889 in Pennsylvania, anti-immigrant rhetoric directed at Hungarians, who made up a portion of the migrant labor force, ran rampant in media coverage. Hilpert argues that such images were the product of "people's fears of the city in the latter half of the $19^{\text {th }}$ century."

\section{Jeanette Gabriel}

Jeannette Gabriel is a PhD candidate in Social Studies Education at the University of Iowa, and a fellow at the Obermann Center for Advanced Studies. Prior to her doctoral work, 
she taught history as an adjunct professor at the City University of New York for ten years. She then joined the Smithsonian as part of the Teaching American History program, a grand-funded initiative through the Department of Education. In that capacity, she developed a curricula for American teachers about how to teach images.

Her research projects examine the unemployed worker's movement in the 1930s. She is particularly interested in how the public perceived the movement. While her emphasis has always been in African-American and labor history, her predominant focus as a history educator is on constructing a body of images that demonstrate what she calls "racial tensions" throughout American history. In her approach to historical work, Gabriel mentions an explicit intention to use photographs as primary sources of evidence "because historians only use them as supplementary to their records."

\section{Jennifer Black}

Dr. Jennifer Black is an Assistant Professor of History and Government at Misericordia University in Dallas, Pennsylvania. She focuses on American visual culture and her most recent book project examines $19^{\text {th }}$-century advertising and early conceptions of branding. She considers herself a specialist in "all things visual" in the $19^{\text {th }}$ century. She has been teaching historyrelated courses since 2004, such as both halves of a US History Survey and a university writing seminar.

Black uses various types of digitized images in her research and teaching, including photographs, drawings, prints, lithographs, and engravings. In searching for images, she uses the same techniques in online searching as she does in archival settings. She typically uses online archives to do her "pre-research," locating images of interest prior to an in-person visit. When conducting research, she prefers engaging directly with the physical object rather than a digital 
surrogate so she can better ascertain material features such as tin-type, collodion plate, etc. For teaching purposes, she often uses a digital image as a surrogate because "I don't need to look at it that closely." She currently teaches a university writing seminar on American visual culture, aiming to instruct students in how to look "critically at images as historical sources."

\section{Jerome Krase}

Jerome (Jerry) Krase is a Professor Emeritus at Brooklyn College and has been teaching in the Sociology department there since 1969. His primary area of research is urban neighborhoods, and his methodological approach might best be termed visual sociology. Krase generally attributes his image use to be "looking for things that will illustrate a point I'm making through some other way." Krase had amassed his own archive of photographic images, a combination of him taking photographs as well as saving copies of relevant materials. During our conversation, he expressed frustration at the disciplinary boundaries that might prevent archivists, for example, from attending American Historical Association meetings. For Krase, such cross-pollination would be especially helpful towards making archives more organized and searchable according to historians needs. He explains that "frequently, what I find, is that the places where the archive exists, they're not done the way I'm thinking about them."

Krase favors the use of images in his teaching and presentations "to literally say something or to tell a story that doesn't require text." Over the course of his academic career, he has held a particular interest in trying to understand what it is that people see when they look at photographs. His arguments consider the role that perception plays in how we understand the city landscape. "With my work on minority neighborhoods and stigmatized places and stigmatized people has a great deal to do with images that people have in their minds about what minority neighborhoods look like." 


\section{Jonathan Grunert}

When we first spoke, Jonathan Grunert was a doctoral candidate in Science and Technology Studies at Virginia Tech. He received his master's degree in History and was interested in applying a historical approach to how science and technology interact. His dissertation project examines taxidermy practices in late $19^{\text {th }}$-century and early- $20^{\text {th }}$-century Natural History museums. He uses photographs to demonstrate methodological differences in taxidermy approaches as a "way of seeing how taxidermy is being made."

Grunert primarily uses materials from the Field Museum of Natural History in Chicago, the American Museum of Natural History in New York City, and the Smithsonian's National Museum of Natural History in Washington, DC. His approach to finding images for this project has been to first start broadly with the search term "taxidermy" and then find "some of key actors that show up those pictures." He particularly likes searching archives by subject headings because he can click through to other related content. If he finds an interesting image, he will download it immediately to Google Drive and record an entry for the image in a corresponding spreadsheet.

\section{Kate Sampsell-Willmann}

Dr. Kate Sampsell-Willmann is a professor at the American University of Central Asia in Bishkek, Kyrgyzstan. She is an intellectual historian of the $20^{\text {th }}$ century, specializing in visual culture. She began writing her dissertation in 1998, when the Library of Congress was just beginning to provide dial up access to materials in their collection. The Farm Security Administration Collection was one of the first she used. In reference to that collection, she notes appreciating an earlier model of digital collections where "they put everything" rather than 
curating content, because "that's really what you need to do in order to make a collection valuable to historians."

Sampsell-Willman points out that there are noticeable absences in the online digital archive that reflect historical political biases. She gives the example of being unable to locate digitized photographs of minorities in convict leash-and-chain gangs in the late $19^{\text {th }}$ century, despite knowing they exist in the archive and may even be digitized. She suggests this is an intentional absence due to the biases of state legislatures, who have political rationales for limiting materials made available to historians outside the region.

In 2009, Sampsell-Willman published the book Lewis Hine as Social Critic, which explores the life and work of the social documentary photographer. Using Hine's photographs as text, Sampsell-Willman draws attention explicitly to the story-telling aspects of his work. Sampsell-Willman currently conducts faculty development seminars on how to use images in teaching and writing, and how to teach students to read and write from an image. Cultivating historians' visual literacy skills is a particular concern for her. She regularly encounters mislabeled information, poor search interfaces, and difficulty aligning search keywords to results. She attributes this in part to postmodernism, which she says has "disconnected the document from its author."

\section{Kristen Turner}

At the time of our interview, Kristen Turner had just submitted her dissertation as a doctoral candidate in Musicology at the University of North Carolina at Chapel Hill. She has been teaching as an adjunct faculty member in Music History at North Carolina State University since 2003. Her primary research interests center on opera, American music, and gender. Her 
dissertation examines the popularity and performances of European Grand operas in the United States at the turn of the $20^{\text {th }}$ century.

Turner uses an array of visual sources to show how class, race, and gender figured prominently into American reception, production, and marketing of opera. One of the kinds of photographic images she collects and uses in her research is carte de visites, the $2 \frac{1 / 4}{4}$ by 4 inch

portraits popularized in $19^{\text {th }}$-century photography studios. During her dissertation research, she frequently consulted the New York Public Library's online collection of digitized carte de visites, as well as the George Eastman House and the Harry Ransom Center. As she conducts her research, she will often bookmark different sites of interest for future work. She also frequently downloads and collects images of interest.

\section{Lisa Jackson}

When we conducted our interview, Lisa Jackson was a Master's candidate in History at San Francisco State University. She was preparing to enter the History PhD program at the University of California at Santa Cruz. Her research interests center on gender in the inter-war period from a global perspective. Her master's thesis examined the gendered social culture of the California Communist Party during the 1930s. She credits photographic evidence as helping to transform her research from the more general "I'm interested in their social life" to "I'm interested in the way that they portray themselves in their social life." Jackson came to her research topic after encountering a number of images that seemed to reproduce the same type of representation: Communist men with bandages or black eyes after confrontations with police or vigilantes. 


\section{Patricia McCormack}

Dr. Patricia McCormack is a Professor Emeritus in the Faculty of Native Studies at the University of Alberta and has been teaching since 1994. Trained as an anthropologist, McCormack's research focuses on the ethno-historical implications of the fur trade on Aboriginal peoples in northern Canada, southern Alberta, and Scotland. Prior to her faculty position, she worked as a curator at the Royal Museum of Alberta.

McCormack describes herself as very visually oriented and mentions using photographs to "get my students to see things that I know they won't have seen in their kind of ordinary, regular lives." She began to use images (illustrations and photographs primarily) in her research on the evolution of Aboriginal sled culture, after finding virtually no written sources on the topic. In her exploration of how sled technology in this particular time and place has changed, she has been able to identify numerous visual sources that counteract popular representations. She explains, "So now that I'm starting to work with these images I see all sorts of things that I didn't start to see...I see images in books that are drawings and I can see how inaccurate they are. In other words, whoever did the final image didn't know much about it and they did it the way they thought it would look."

\section{Stephen Gold}

Dr. Stephen Gold is a Professor and Associate Chair in the department of Sociology at Michigan State University. He has been teaching sociology for over thirty years and his primary areas of research center on international migration. He has taught a variety of undergraduate and graduate courses on a range of content, mostly focusing on race, ethnicity, and immigration. He has been an avid photographer since high school and attributes his frequent use of photographs as originating in his own practice. 
In his 2010 book The Store in the Hood, Gold uses a number of digitized archival photographs (some of which had not been accessed publicly prior to their digitization) to investigate conflict between immigrant entrepreneurs and their customer base. In one chapter, Gold uses juxtaposition of, and original captions from, different photographs to make the argument that the 1940s Japanese interment in part stemmed from xenophobic anger in earlier decades over the success of Japanese entrepreneurships. Gold explains, "The particular threatening of small business, that it's not so much about war or Pearl Harbor, it's about 'We don't like these people making a decent living in our country,' kind of. They're not one of us." As a sociologist by training, Gold tends to compare group behavior to situate his historical arguments.

\section{Wendy Clark}

Wendy Clark is a PhD candidate in the department of History at the University of Memphis. She is an environmental historian of the American South in the $19^{\text {th }}$ century, and tends to teach survey courses on US History prior to 1877 . Her dissertation examined how one particular community in Memphis - Catfish Bay - interacted with the Mississippi River from 1820 until 1880.

Clark describes her research process as beginning with Worldcat and focusing specifically on archival materials. She prefers not to use Google image searching because "it is not very clear where they get their images from." Authority is a term she mentions often in relation to her use and evaluation of photographs. As part of her dissertation research, she used photographs pieced together with maps and other textual sources to ascertain the probable location of Catfish Bay. 


\subsection{Descriptive and Analytical Themes of Historians' Experiences}

One aim of this research was to investigate qualitative aspects of historians' experiences using photographs, to reveal the factors that matter to them in their image selection. At the same time, I wanted to analyze actual examples of use to understand the various ways in which historians were using digitized photographs to make arguments. Using thematic analysis and thematic synthesis, I identified seventeen descriptive themes and six analytical themes (see Figure 3). Taken together, they can be used to conceptualize aspects of historians' experiences using digitized photographs as evidence.

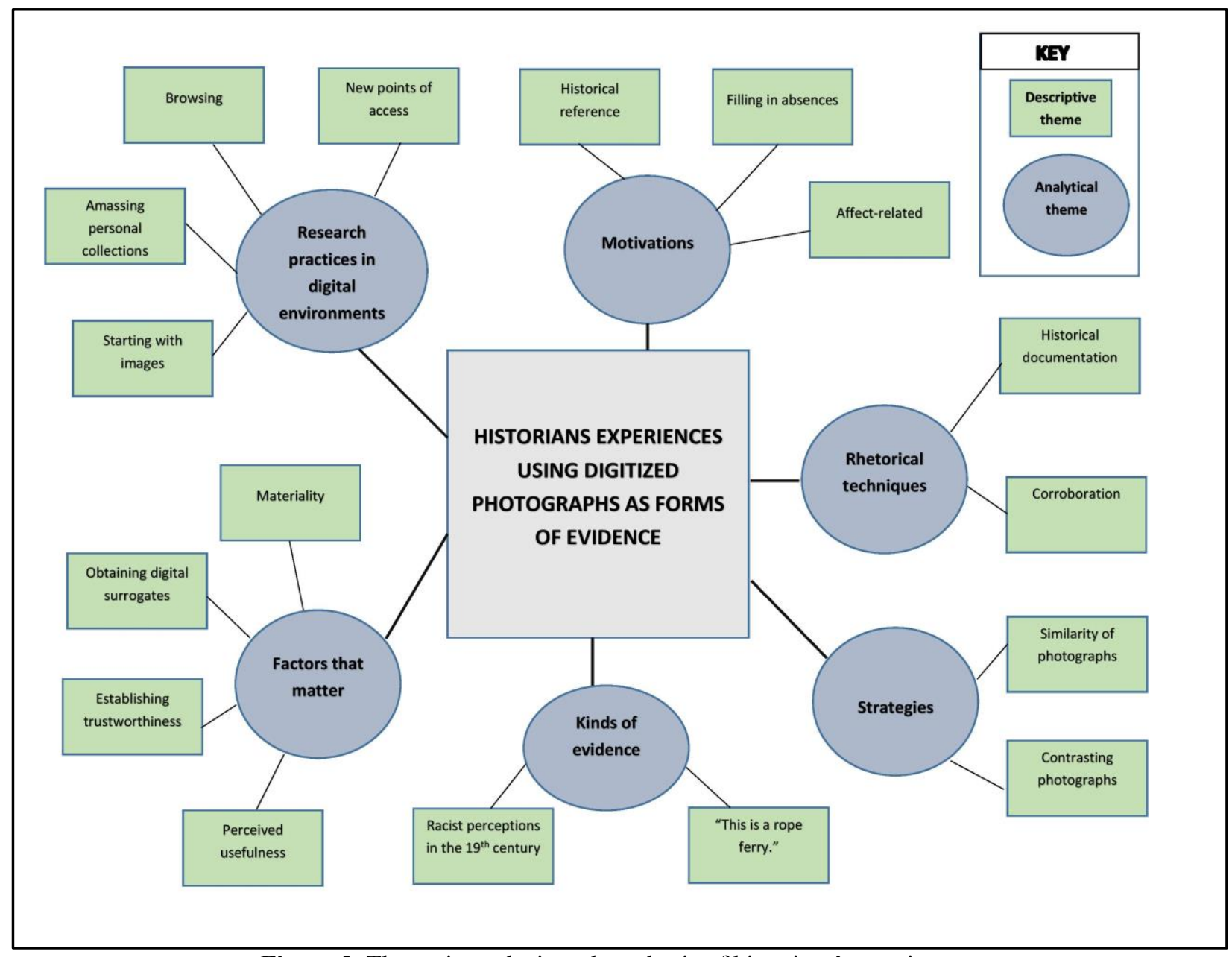

Figure 3. Thematic analysis and synthesis of historians' experiences 
The first analytical theme I identified is research practices in digital environments, where respondents describe themes related to how they conduct research in digital environments. They might mention functional aspects of the research process, such as the search terms they use or the digital tools they consult. A second analytical theme concerns their motivations for selecting images; several participants mention that photographs can evoke powerful reactions from viewers. Other motivations to select images include the ability of photographs to "show things the way they were." Participants also mention a variety of different factors that matter to them as they interact with digitized photographs. For instance, are they able to download images easily? How expensive is the cost of reproduction?

In analyzing examples of image use ${ }^{341}$, I was able to identify how my participants use different strategies to position photographs as forms of evidence. For example, one participant developed his central thesis after noticing the frequency with which he encountered photographs of families from the turn of the century posing with musical instruments. He explains how and why he used photographs from digital collections as a strategy in making his argument.

But to really bring home the points that I was making, I started bringing in other images that were taking elsewhere. Otherwise, I would be portraying this as something that was just happening in Alabama, which of course it wasn't. And so my use of digital images really comes into play- Well, I have two thoughts on that. One is looking for similar pictures across the United States, which I wouldn't have been able to do without being able to access digital archives. And this is probably useful, although it's pretty lame and boring, but I did a lot of Google image searches. And spent a lot of time sort of honing how I did that and to come up with a lot of images like the one I sent you of those musicians in Kansas.

My participants also use rhetorical techniques in their arguments to support their use of photographs as evidence. Rhetorical techniques range from ascribing intentionality to

\footnotetext{
${ }^{341}$ As exploratory research, the aim of this study was to uncover some of the factors underlying practices and processes in historians' use of digitized photographs. Therefore, a full historiographical account of historians' construction of evidence is outside the scope of this research. Instead, this study investigates evidential use as discursive practice and suggests aspects of the experience that can be used in future research.
} 
photographic subjects based on the way they represent themselves visually, to making claims about how the general public might interpret an image. The final analytical theme I identified is different kinds of evidence, or the descriptive themes my participants use in identifying photographs as being representative of or about something. For example, one participant uses photographs as representative evidence of what a $19^{\text {th }}$-century dog sled actually looks like.

Another participant uses photographs as documentary proof of racist perceptions in the $20^{\text {th }}$ century.

\subsection{Potential Image Use}

One goal of this research was to discover dimensions of historians' experiences that influence them in their image use practices. I noticed how certain themes related to image selection seemed to be an important aspect of historians' experiences. Conceptualizing a separate sphere of activities related to potential image use helped me to surface themes related to historians' interpretive and evaluative processes during image selection (see Table 4).

\begin{tabular}{|c|c|}
\hline ANALYTICAL THEMES & CODING EXAMPLE \\
\hline Research practices in digital environments & $\begin{array}{c}\text { "And I contacted a man who had a great digital } \\
\text { scan of this image and the rights to it and he was } \\
\text { nice enough to let me use it, sent me permission } \\
\text { and things like that. }\end{array}$ \\
\hline Motivations & $\begin{array}{l}\text { "But I'd always try to find good touristy photos } \\
\text { to show some good } 60 \text { s and } 70 \text { s touristy photos } \\
\text { or whatever, just to make it livelier." }\end{array}$ \\
\hline Factors that matter & $\begin{array}{l}\text { "It's important to know where it's from and when } \\
\text { it was created and what the archive knows about } \\
\text { it, which certainly guides me, and it's important } \\
\text { to know where it is so that I can know who to } \\
\text { contact to get the high-quality image." }\end{array}$ \\
\hline
\end{tabular}

Table 4. Analytical themes in potential image use 


\subsubsection{Research practices in digital environments}

As discussed in the literature reviewed in section 2.3, image use is intricately tied to research practices. Lori McCay-Peets argues that image use is often "tightly integrated with the work task that triggered it, such as finding an image to explain a concept in a presentation, or adding visual appeal to an article." At the same time, the literature reviewed in section 2.1 suggests that historical research practices are currently in flux, due in large part to technological advancements. The extent to which such changes are impacting research practices is not wellunderstood and only beginning to be explored.

My participants describe five themes related to their research practices in digital environments (see Table 5). Each theme is discussed in greater detail below with illustrative examples from different cases.

\begin{tabular}{|c|c|}
\hline THEMES & \#PARTICIPANTS \\
\hline Browsing & 15 \\
\hline Starting with images & 6 \\
\hline Orienting & 6 \\
\hline Amassing personal collections & 5 \\
\hline Different entry points for access & 10 \\
\hline
\end{tabular}

Table 5. Research practices in digital environments

Browsing. All fifteen participants mention that they engage in browsing in their research or instructional pursuits. They described two different kinds of browsing. The first form, what I am calling "whittle-then-browse," refers to when my participants initially limit their search results, and then cycle through images to try to locate material. Sampsell-Willman explains that, "I wouldn't do keyword searches, because I know that keyword searches are going to limit what I find. And if I'm looking for everything, you know what I mean, if I don't know what I'm going to find yet, then I don't want to limit what I see.” The second form of browsing is primarily exploratory and tends to be motivated by searching for generic, representational images. When 
Black is browsing in this mode, she says that she "might have been looking for shop signs in New York, and so any image that had shop signs in it would be okay."

Starting with images. Six participants began their research projects with an intention to use photographs. Some participants elaborated that this was deliberately in opposition to how historians typically use images - as supplementary illustration in support of an existing argument. The extent to which this practice is markedly different from research practices in physical archives is not known. However, the historiographical literature reviewed in 2.4 suggests that photographs are most often used as illustration rather than as primary sources of evidence.

In her research, Jeanette Gabriel describes how photographs are "the foremost piece of evidence that would then be corroborated by written record[s]." She uses images "as a starting point to investigate what had happened in that historical moment." Andrew Nelson concurs: "I'm not writing history and then using images to illustrate what I'm writing. It's the other way around. So I'm starting with the images, and I'm thinking 'okay, what if we start by sort of thinking about these historical questions with the pictures and then see where that takes us?" Similarly, Zachary Hilpert went into his research project just being "fascinated with the images" and then looking to see "what story would develop out of it."

Orienting. Six participants mention that photographs orient them early on in their research process in two distinct ways. First, accessing images online helps them determine whether or not to visit a repository in-person. Second, participants may download images they simply find interesting at an early stage of research, presumably for use at a later point. For example, Kristen Turner explains why she might download photographs in the exploratory research stage. 
My experience is that there are sort of two types of research that I was doing. One was just the sort of, who are these people- I'm researching an area of musicology that is relatively untouched- so I didn't know who any of these people were. I mean, I really had to get the lay of the land. So, I did not know, initially, who was going to be important and who wasn't in my research. So, there was that sort of- and that went on for a very long time, even when I thought I was doing more specialized research, it turned out to beand then, there was. . . Now I'm working on the Emma Juch chapter, right? And so I would collect all the images I could find of Emma Juch, who's an opera singer, but I wouldn't necessarily realize until later, "Oh, this is the interesting image," you know, you see what I'm saying? So in both cases, both whether I was doing some more general kind of orientation research, or if I thought I was doing something more specialized, I usually didn't realize right away which images were going to stick out.

Amassing personal collections. In addition to the growing number of materials digitized and made accessible by libraries and archives, my participants are beginning to amass their own collections of materials in digital form. In large part, this can be attributed to the ease with which digital objects can be personally acquired and stored on personal computers. ${ }^{342}$ Five participants mention collecting digital materials for research and/or instruction through three methods: 1) the use of portable digital cameras in archival reading rooms and in the public sphere; 2) downloadable digitized online sources; and 3) home scanning equipment.

Different entry points for accessing materials. Digitization has undoubtedly influenced how and where historians are accessing photographic materials. Ten respondents describe locating images outside of the digital archive. The formerly bounded nature of visiting an archival repository, interacting with an archivist, and receiving boxes of photographs to browse through has been transformed into an experience with vastly different dimensions. Historians can now view and assess digitized photographs on their own time and in various locations.

\footnotetext{
342 The practice of acquiring and managing digital objects for personal use has come to be known broadly as personal digital archiving, in which "individuals manage or keep track of their digital files, where they store them, and how these files are described and organized." See: Gabriela Redwine, Personal Digital Archiving, DPC Technology Watch Report 15-01, 2015, http://dx.doi.org/10.7207/twr15-01.
} 
Eight respondents mentioned that they regularly use Google image search to locate items of interest, though most noted they would then pursue a version of that image held by an archival repository. In her work with educators, Gabriel encourages teachers to use archives for image searching so that contextual elements are not lost "because history teachers are just picking up stuff from Google and taking it out of blogs." Jennifer Black points to the Library of Congress as a model archival interface for providing links for items back to their collection.

Historians are also using digitized materials put online by individual collectors or hobbyenthusiasts. Five respondents located digitized photographs from amateur collections for use in their research or instruction. This trend was noted by Melissa Terras in 2010, who writes, "Enthusiastic digitisation by amateurs, a phenomenon previously ignored by information professionals, is providing a rich source of online cultural heritage content which often documents areas not covered via traditional institutions." 343

At the same time, images that are dislocated from archival context, such as those found on blogs or crowd-sourced sites like Wikipedia, introduce additional challenges for historians who are used to relying on the archival repository for authentication and sourcing of materials. One participant discovered first-hand the complexities that can arise from locating an image online. In a Power-Point Presentation for a class, she used what she thought was a digitized photograph of Anna Held, a performer from the early $20^{\text {th }}$-century. She initially found the photograph via Google Image Search, on a blog dedicated to vintage photographs of women at the turn of the $20^{\text {th }}$-century. The original photograph, digitized as part of the Macauley Theatre Collection at the University of Louisville Photographic Archives, tells a different story.

\footnotetext{
${ }^{343}$ Melissa Terras, "Digital Curiosities: Resource Creation via Amateur Digitization," Literary and Linguistic Computing 25, no.4 (2010): 436.
} 
According to its title, the woman pictured in the photograph is actually one of Anna Held's fencing girls. ${ }^{344}$

\subsubsection{Motivations in potential image use}

My participants report being motivated to potentially use images by three main themes (see Table 6). Affect-related themes, such as the ability of images to provoke reactions, play a significant role in motivating participants. Another motivating theme concerns the ability of images to act as historical reference in showing "the way things were." A third motivating theme that drives my participants is a desire to fill in absences for under-represented populations and/or stories.

\begin{tabular}{|c|c|}
\hline THEMES & \#PARTICIPANTS \\
\hline Affect-related & 9 \\
\hline Historical reference & 10 \\
\hline Filling in absences & 4 \\
\hline
\end{tabular}

Table 6. Motivations in potential image use

Affect-related. As is often the case with visual experiences, there are elusive qualities that motivate my participants to select (or not select) images. As noted by Barbara Orbach, photographs "can convey a mood or the sheer visual impact of a situation in a way that few other types of documents can." ${ }^{345}$ Nine participants describe affect-related dimensions as motivating factors for image selection. For example, three participants point out how they might use photographs because of a "certain something" that caught their eye. Salo explains how in developing course materials, photographs "make it more exciting for students." For Gabriel, the anticipated impact of certain historical images became central to her instructional training with teachers:

\footnotetext{
344 See: http://digital.library.louisville.edu/cdm/ref/collection/macauley/id/1377

345 Barbara Orbach, "So That Others May See So That Others May See: Tools for Cataloging Still Images." Cataloging \& Classification Quarterly 11, no.3-4 (1990): 164.
} 
So a lot of what I was doing, in terms of training teachers, was trying to convince them to teach controversial issues that they didn't really want to teach. And teachers have a lot of autonomy, you know, they close the classroom door. Race is not a mandated topic in American history. You can skim over African-American history and teach very little. So I was seeking buy-in, and I felt that maybe the images would foster empathy.

Nelson notes how the uniqueness or unusualness of an image might drive him to use it in teaching. He says, “Images that I think often times grab people's attention are ones that are very different from ones they see on a daily basis." In his description of image selection practices, Daegan Miller notes, "I look for anything that catches my eye. And I don't always know what I'm looking for, but I'm looking for anything interesting and unusual.” Lisa Jackson concurs with this sentiment, saying "if it says something to me - if it has an interesting take on gender representation is pretty much the first thing I'm looking for." In this way, photographs can act as provocations for historians, prompting them to pursue deeper exploration.

In some cases, emotional responses to photographs can help translate historical concepts for students. Kristen Turner is a musicologist who uses photographs to teach about the musical history of Jim Crow and minstrel shows. She describes how photographs engage her students in ways that listening to music often cannot.

You know, if we're listening to music from minstrel shows, they're very, very racist, horrifically racist, but college students are always really in tune to- they don't understand the racist images- or the racist language- they don't always understand that "Jim Crow" is a racist thing, or whatever. But you see the image of Jim Crow, that's really obvious. And then when you hear it, and so then I can say, "Well, this image, you can see how racist this is, this is easy to understand." But in the music, they get sort of caught up in how can music be racist, and how can you have musical markers for racism, and how is this language racist?

Historical reference. Ten participants consult digitized photographs for verification, documentation, or corroboration. However, there are distinctions in how they approach photographs as sources of historical reference. Six participants mention that photographs are 
helpful for contextualizing their understanding of a specific time and place. For example, in her research on late $19^{\text {th }}$-century advertising and early conceptions of branding, Black describes the potential usefulness of photographs to help flesh out "what things looked like then." She explains how historical streetscapes offer a valuable perspective to her project because they show "what the shop front looked like, to know what the point of sale looked like." Similarly, Wendy Clark describes how finding historical streetscapes of Memphis helped ground her research geographically, offering her "some kind of a real image of what was going on" at the time.

Four respondents note that photographs can serve as reference in contemporary use, by providing essential historical details about specific time periods and habitats. Edward Roach, a public historian at the Dayton Aviation Heritage National Historical Park, uses digitized archival photographs originally taken at the home of Orville and Wilbur Wright in 1948 to recreate historically accurate details about the home, including furnishings and layout. Similarly, in his work as a consulting historian on historic preservation for a cultural resources management firm, Edward Salo uses visual materials such as historical photographs to justify his restoration recommendations. He explains:

I try to use a lot of historic images if I can, to illustrate what buildings used to look like. Especially where we're studying areas where you can do some of those streetscapes to show how. One example was the town we live in here, Jonesboro, Arkansas. We were doing a walking tour of downtown, and I had found some images from our local public library that were from the 1920s. And I just printed them out, just so I could show them. I wasn't sure, you know it said "Main Street, south view, circa 1923," you know I wasn't going for any specific buildings, just kind of an overview. And you could see power lines, telephone poles and everything, right along one of the sides of the street. And a lot of times preservationists are like, "We can't have this, it clogs up the view," and I'm like, "Here it is back in '23, and there's power lines right down the middle." And you know, a lot of times, that's what preservationists would be attacking, and if you look now, there weren't any poles in the downtown at all, because most of the stuff has gone underground. So I was like, "So. .." A lot of times we need to look at these historic photos to actually know what it actually looked like and not just our idea of what it looked like. 
Filling in absences. Participants also point to the ability of photographs to potentially fill in absences found in the historical record. As the literature in section 2.4 notes, there is growing acknowledgment that multicultural perspectives are missing from the so-called "Great Story" of history. Four participants mention being motivated by a desire to help fill in under-represented histories. For example, Gabriel describes how in her teaching and research, she has attempted to use photographs that "documented sort of unseen moments in American history, that were not in the historical record, and then use that as a starting point to investigate what had happened in the historical moment." Her master's thesis examines the underrepresented story of African-

American women participating in the St. Louis nut processor's strike in the 1930s.

So I knew that this strike had happened, but to find the images really drew me in deeper. Because the way African-American women were able to go out on strike, is they aligned themselves with the unemployed councils, which were black women in the neighborhood. And white women in the neighborhood. So, they got white women to come to their picket lines. So when the white women saw the strike going on, they saw white women out on the picket line and joined the picket line. And it was images of that that really drew me into the fact that there was a whole, much more extraordinary history linked to the power of images, that hadn't been told before.

Gabriel also spent nine years traveling around the country as a participant in Teaching American History, a federal grant program through the Smithsonian designed to fill in the gaps of knowledge of American history teachers.

\subsubsection{Factors that matter in potential image use}

Presumably, a number of factors influence how and why historians decide to select and use images. According to Maxwell, historians who use digital libraries to conduct historical research have "disciplinary idiosyncrasies that colour their interactions with digital sources." 346 I

\footnotetext{
${ }^{346}$ Alexander Maxwell, "Digital Archives and History Research: Feedback from an End-User." Library Review 59, no. 1 (2010):24-39.
} 
identified five themes that mattered to my participants in their interactions with digitized archival photographs (see Table 7).

\begin{tabular}{|c|c|}
\hline THEMES & \#PARTICIPANTS \\
\hline Materiality & 6 \\
\hline Ability to obtain digital surrogates & 7 \\
\hline Trustworthiness & 9 \\
\hline Perceived usefulness & 11 \\
\hline
\end{tabular}

Table 7. Factors that matter in potential image use

Materiality. My participants noted distinctions between their interactions with digital surrogates and their analog counterparts. Overall, six respondents state a particular preference for digital or analog formats (four prefer digital; two prefer analog). Some of the affordances that respondents mention as beneficial for interactions with digital surrogates include the ability to see greater details than would be possible in an analog counterpart. Despite what SampsellWillman refers to as the "wow factor" of holding an archival photograph, she still prefers a digitized surrogate:

I would say that it's actually better on the screen, largely because...there's a skewing of the details. There's more details on the screen then there is in the photograph. Because, unless you get really close or you have a really good eye, or you're allowed to touch the photograph, you're not going to be able to see everything that's there. Which means that the people who originally saw the photograph - you're actually seeing the level at which the photographer saw. Which I think is better. Better than...I mean, of course the original photograph is beautiful as an object.

For Hilpert, interacting with a high-quality surrogate of an early $20^{\text {th }}$-century photograph mimics what he anticipates as the experience "that probably somebody who was holding the physical copy could have had a hundred and thirty years ago.”

At the same time, two participants describe their preferences for interacting with an analog photograph after initially viewing it digitally. Miller ruminates on seeing an image digitally that piqued his interest, leading him to follow with in-person examination:

And I was like, oh that looks cool and mysterious, that sort of speaks to me somehow, I want to make sure I look at the real thing when I get there. So I don't want to say that the 
digital was not helpful, or that it misled me or anything. It actually led me to the physical image. But yeah, I'm thankful I got to hold the hard copy in my hand.

Also, as Black points out, often times the digital surrogate may not provide enough information about an image, particularly for scholars interested in material aspects of a photograph's production rather than its viewing consumption.

Ability to obtain digital surrogates. There are also practical factors that matter to my participants as they consider further use of images. Seven respondents describe the reproduction process as a potential obstacle for them. One specific concern they share is the cost of obtaining and/or reproducing a digital surrogate. Turner describes how she decides to select images for use in publication:

Eastman will give me permission at a very high cost. But, the editors of the volume, I don't know what their, what they need to do with their publisher, right? Like... are they going to have any restrictions on how many images can be printed in the book, that kind of thing... so, I don't know anything about that, yet. And I sort of just, I did what my advisor said, which was to send it all in like, "of course you're going to do this," and then, you know, find out later if I have to make a choice, like, "oh, we'll let you do one," or something like that. So this would be the one that I would definitely use if I had to make a choice, though.

Cost also factors into the ability to obtain surrogates. If the image hasn't been digitized the ability to pay for digitization and use (if necessary) can be prohibitive for my participants.

Additional obstacles include confusion or difficulty with obtaining copyright

permissions. Some institutions are more transparent and straightforward about their process. Hilpert explains:

One of the things that is nice about the Library of Congress website is use. So if I were to, for example, use this image in an article that I were going to send out, getting permission to use this image is incredibly simple because it's owned by the Library of Congress. If it's another kind of private institution or something, then there's a lot more agreements that have to be made, and rights questions that you have to ask, but being that it's from the Library of Congress and its owned by the US government, they make it available for use, basically for free. So that's certainly useful. 
Despite potential difficulties in obtaining reproductions, participants stress the overwhelming value of access to digitized surrogates. Online archives provide the opportunity to locate images easily, without necessarily having to visit an archival repository. This can help scholars narrow their image selection considerably, making the complex aspects of reproduction more manageable. Nelson describes how online archives helped him to find useful images for his dissertation:

I didn't have the time or money to plan a trip to Kansas. I wouldn't have even known I wanted to go to Kansas until this was digitized and put online. It really struck me, when I got to the end of my dissertation and was giving permissions to all the archives I used that, you know, I'm calling people in Kansas, in Utah, Wisconsin, all of these [unintelligible] historical societies all over the country to get permissions just for one photo. So that to me, really, is a testament to the accessibility that has come with digitization of images.

Trustworthiness. Respondents need to be able to trust sources in order to use them further. The reputation of an institution, as well as the simple fact that an image originated from an archival institution, plays an important role in establishing trust for nine participants. Salo describes how he tries to stay as close as possible in his research process to "reputable repositories, be they national archives or a state archive, or a good University." The notion of the archive as a harbinger of truth repeatedly surfaces in respondents' descriptions; Black states, "I generally tend to take what the archive tells me as truth." Certain archival institutions might carry more weight for my respondents, though this might be attributed to comfort based on previous experiences. Turner explains:

Well, I have to say if it's New York Public Library, I trust it. You know, obviously an image that's on some random website that's come off of Google image, I would be more careful with using. But if it's a University website, you know, University library, New York Public Library, something like that, the Historical Society of D.C., then I pretty much trust that what's there is what's correct. So I would say I don't usually second-guess those sources, because I trust the repository. 
Additionally, personal relationships with archivists can also contribute to trustworthiness. Clark mentions being more willing to use a photograph in her scholarship because she knows the archivist who helped to digitize it, as well as the original donor's family.

Perceived usefulness. The LIS literature examining visual material use suggests that one construct which can be used to examine potential use of materials is perceived usefulness. ${ }^{347}$ In this study, perceived usefulness as a construct helped to unify the varying techno-social dimensions that cut across participants' experiences in digital research environments (see Table 8).

\begin{tabular}{|c|c|c|}
\hline THEME & POTENTIALLY RELEVANT CHARACTERISTICS & \#PARTICIPANTS \\
\hline Provenance & Title, photographer, location of original item & 7 \\
\hline Contextual elements & Subject headings, keywords, captions & 4 \\
\hline Artefactual qualities & Medium, size, format & 5 \\
\hline Functionality / usability & Ability to zoom, ability to download & 7 \\
\hline Quality of digital surrogate & Resolution, ability to be projected & 6 \\
\hline
\end{tabular}

Table 8. Perceived usefulness as a construct

Provenance plays a critical role in how my participants evaluate images as evidence. ${ }^{348}$ Identification of the original photographer, title, and related captions are all important sources of information. Conversely, learning that the original photographer is unknown is also useful. For Roach, knowing an item's provenance is directly related to potential use. He explains, "It's important to know where it's from and when it was created and what the archive knows about it, which certainly guides me, and it's important to know where it is so that I can know who to contact to get the high-quality image."

\footnotetext{
${ }^{347}$ For in-depth examinations of perceived usefulness of visual materials in LIS, see: Kathleen Fear, "User Understanding of Metadata in Digital Image Collections: Or, What Exactly Do You Mean by 'Coverage'?" The American Archivist 73, no.2 (2010): 26-60; Ying Zhang and Yuelin Li, "A User-Centered Functional Metadata Evaluation of Moving Image Collections," Journal of the American Society for Information Science and Technology 59, no. 8 (2008): 1331-46, doi:10.1002/asi.20839.

${ }^{348}$ In their glossary of archival records and terminology, The Society of American Archivists defines provenance as "information regarding the origins, custody, and ownership of an item or collection." See: http://www2.archivists.org/glossary/terms/p/provenance\#.V0tJHPkrK00
} 
Interestingly, participants seem to feel more authoritative about using surrogates when they know the original archival location because "if I need to find the physical copy, I can." Information about the photograph's origin also makes my participants more comfortable with using photographs in their teaching. Miller points out how provenance plays an important role in his decision to select photographs for use in instruction:

And then the other factor is - is it easy to track down the citation- the photographer, when it was made, where it's being held, so that a) I can practice "good citation practices" for my students, but also b) if they're interested, they can go find the image themselves. And a lot of my assignments- with most of my classes, I'll offer an assignment that says "Use these images as primary sources. Make an argument about the brutality of the Civil War," or something like that. Whatever the case may be. But I want to make sure to give them the information they need to find the images online for themselves, or in the library, or wherever.

Contextual information, such as related keywords and subject headings, influences

participants. In his study of historical taxidermy practices, Grunert points out how keywords are useful for locating figures of interest:

I've started out by looking at just taxidermy, and using that to get the broadest range that I can find. But, of course, that one keyword doesn't help with everything. So, once I've used taxidermy, I've been looking at some of the key actors that show up in these pictures, and then looking them up by name, then. So, still doing subject searching, but looking at it through the lens of Carl Akeley or William Hornaday, or whoever I might find.

Photographic captions can also be used to provide historical snapshots of a particular time period. For example, Stephen Gold describes how a photographic caption functioned as contextual evidence in his larger argument about community perceptions and social norms.

And so I found, in the FSA archives, a photograph of a Greek ice cream store owner that was taken in the late 30 s or early 40 s, and the caption explicitly said that he was a US Veteran. So, I took this as- you know, this idea of Greeks were not patriotic enough, and they were just making money from their businesses and not fighting in the military- had shaped this caption of a photograph from the FSA, you know, twenty years later. So, that kind of thing. So, I didn't intentionally use it to make that argument, but once I connected those things together, I was able to use that, and use the caption of the photograph as empirical evidence." 
Of course, respondents recognize that assigning relevant keywords and subject headings has significant influence on how findable materials are in the first place. Jackson points out:

One of the stumbling blocks is really the keyword search, with visual images. How many words, how do you figure out what words are going to be relevant for a photograph, for researchers? Because sometimes it's kind of hard to imagine what other people might find in this image, when it's not your particular research, you know?

Artefactual qualities about the original source material also influence respondents' perceptions of usefulness. Five participants mention the importance that descriptive characteristics play in deciding how and whether to use an image. Factors such as the original medium, size, or format play a significant role in evaluation. For example, Nelson describes how material aspects influence his interpretation:

What the edges tell me is that it's a glass plate negative - something that would interest me would be did they scan the negative, or was it a print made from the negative, and did they scan that?...I think it was a print made from the negative that they scanned, just because you can see how the paper's kind of rolled on the right hand side. But yeah, I love the sort of material aspects of it as well and in a lot of ways think of a negative like this... as not just a photograph but also an object.

It can be useful to consult the original source material in person as well. Roach describes how glass plate negatives (from which historic prints were made and later digitized) offer important historical distinctions.

Though one thing that has mattered, especially with some of the negatives over the years, was that the Wrights stored them in their old bike shop in Dayton, and there was a huge flood in Dayton in 1913, and the negatives were under water. So some photographs that you see from these glass plate negatives, if you see prints that were made before the flood, they look like fine, normal prints. If you see the same negative after the flood, it's splotchy and damaged.

A fourth area that influences my participants' perceived usefulness is the functionality of an image. Factors such as the ability to download different versions of photographs or being able to zoom in to see greater details are important considerations in image selection. Salo 
summarizes some of his favorite functional aspects about the American Memory Collection, at the Library of Congress:

I really like the different ways you can download things from their website, just depending on what kind of graphic needs and how you're going to utilize them.

Sometimes you just want to get the low-res thing just for comparing, or you want to go with a large .tiff kind of thing for a presentation.

Finally, the quality of the digitized surrogate plays a role in perceived usefulness for my participants. Some considerations are practical; Miller wonders whether a digitized photograph will project appropriately in the classroom. Notions about quality can also be akin to scholars determining if they can accurately perceive specific details in photographs. Turner points out how even “online sometimes, the images just don't come across very well...you can't see the sort of detail you want." Jonathan Grunert concurs, explaining how "resolution of image matters a whole lot, especially when I'm trying to find some things that I might want to use in a presentation."

By the same token, participants recognize that the use of digitized surrogates helps to preserve fragile materials. Jackson describes her appreciation of the "rogue scholar" who took it upon themselves to digitize frail historical materials residing at the Labor Archives and Research Center. She notes how her experience was vastly improved working with digital surrogates.

They have hard copies of a lot of the newspapers at LARC, but they're falling apart. There's great interest in 1934, in particular, and so every time you open up the book, it's just, bits and pieces are falling out all the time, so it's really nice that people are taking it upon themselves to do this, it's a lot better than microfilm.

\subsection{Evidential Image Use}

The use of photographs in research dates back to the first decades of the twentieth century. Anthropologists began using photographs as ethnographic media, a practice which helped solidify and demonstrate their capacity to serve as cogent research materials. Writing in 
1959, the anthropologist John Collier Jr. asked the following of photographs: "Why could we not consider the photograph a cultural map that could be read with equal clarity by the knowing informant? Could this process allow us to analyze the contents of photographs - and therefore of situations - that we had not previously understood?" 349

In this section, I discuss specific examples of how and why my participants use digitized photographs as evidence in research and instructional capacities. The following research question guided this stage of analysis and interpretation.

Sub-RQ1: How are historians using digitized archival photographs as evidence in their research and instructional activities?

In this portion of my analysis, I used photo-elicitation interview transcripts and research products (e.g., journal articles, conference power-point presentations) to understand evidential use.

I identified three analytical themes that take place during evidential image use. My participants employ strategies (e.g., juxtaposing images with text) to lay out their arguments, use rhetorical techniques (e.g., corroboration) to situate their historical argument, and assign kinds of evidence (e.g., racist perceptions in $19^{\text {th }}$ century America) to images to make their argument (see Table 9). Taken together, these interactions culminate in what I'm calling "modes of historical evidence.” Integrating participants' actual examples of use with the descriptive themes surfaced in my initial thematic analysis helped to triangulate my findings.

\footnotetext{
${ }^{349}$ John Collier Jr., "Photography in Anthropology: A Report on Two Experiments," American Anthropologist 59 (1957): 843-59.
} 


\begin{tabular}{|c|c|}
\hline ANALYTICAL THEME & EXAMPLE \\
\hline Strategies & Display contrasting portrayals from similar time periods \\
\hline Rhetorical techniques & $\begin{array}{c}\text { "And there's a scene, the scene there, you know, very nice } \\
\text { simple street, no people on it at all. And that's to make a } \\
\text { point, too, because when a black person appears on a } \\
\text { street it changes the perception of the place. These are all } \\
\text { visual arguments." }\end{array}$ \\
\hline Kinds of evidence & $\begin{array}{c}\text { "So, I took this as- you know, this idea of Greeks were not } \\
\text { patriotic enough, and they were just making money from } \\
\text { their businesses and not fighting in the military- had shaped } \\
\text { this caption of a photograph from the FSA, you know, twenty } \\
\text { years later." }\end{array}$ \\
\hline
\end{tabular}

Table 9. Characterizing evidential image use

\subsubsection{Constructing modes of historical evidence}

How do images become evidence? In her Notes on Photography, Susan Sontag describes the various ways in which photographs can be utilized as forms of evidence - how they can "incriminate" as well as "justify." The dual nature of photographs, what Marcus Banks refers to as "to be and to represent", complicates attempts to understand characteristics of information use. ${ }^{350}$ Nearly thirty years ago, the librarian Sara Shatford-Layne proposed that indexing approaches for describing visual materials needed to account for the fact that they can be simulatenously "Of" and "About." She writes, "What an image is $O f$ is perhaps more likely to be concrete and objective, while what an image is About may be more likely to be abstract and subjective." ${ }^{351}$

In their construction of evidence, historians can choose to invoke photographs at either the "Of" or the "About" levels; both have implications for how images are used in their

\footnotetext{
${ }^{350}$ Banks, The SAGE Handbook of Qualitative Data Analysis, 394.

${ }^{351}$ Sara Shatford-Layne, "Some Issues in the Indexing of Images," Journal of the American Society for Information Science 45, no. 8 (1994): 584.
} 
arguments. Interpretive readings at the "About" level likely produce descriptions in the realm of “symbolic readings and abstract concepts." In contrast, interpreting photographs at the "Of” level will likely result in descriptions of "people, places, objects, conditions, and actions that have a physical manifestation." 352 The historian who interprets a photograph as being representative $O f$ something (e.g., racist perceptions in the $19^{\text {th }}$-century) will likely have a different path towards how they use the photograph in the construction of their argument, than the historian interpreting a photograph as being About racist perceptions in the $19^{\text {th }}$-century.

Indeed, as the literature in 2.4 suggests, historians inherently apply their own ideologies to perceptions of evidence in the construction of historical arguments. As Berkhofer explains, "The problem with historical facts, as with histories themselves, is that they are constructions and interpretations of the past. Evidence is not fact until given meaning in accordance with some framework or perspective." ${ }^{353}$ Analyzing examples of use through the discursive strategies of thematic analysis and synthesis allowed me to chart respondents' construction of their own "Great Story" - what I'm calling modes of historical evidence. Through a combination different strategies and rhetorical techniques, historians position photographs as being representative of different kinds of evidence.

\subsubsection{Case vignettes}

Below, I present different case vignettes that describe how and why my participants chose to use digitized photographs as forms of evidence in their instruction and/or research activities. Each vignette seeks to illustrate in greater detail how each participants uses images in their construction of different modes of historical evidence.

\footnotetext{
${ }^{352}$ Shatford-Layne, "Analyzing the Subject of a Picture," 45.

${ }^{353}$ Berkhofer, 53.
} 


\section{Using photographs as historical evidence of the photographer's vision}

There is widespread acknowledgement that photographers play a role in both the production and consumption of photographs (choice of subject matter, framing, positioning). Writing in her 1977, Susan Sontag asserts, "Even when photographers are most concerned with mirroring reality, they are still haunted by tacit imperatives of taste and conscience." ${ }^{" 354}$ In his research argument, Miller focuses on a stereographic triptych taken by Eadweard Muybridge at the Thousand Mile Tree, a marker for the thousandth mile of track laid west on the Transcontinental Railroad.

Miller uses these three photographs along with historical texts to contextualize his argument that in shooting these images, Muybridge was intentionally creating a narrative of historical drama. While there are numerous details in each image, he points out how in the first image (see Figure 4), if you zoom in enough you can see that propped against a black box is a hatchet with a tomahawk.

354 Susan Sontag, On Photography (New York: Farrar,Straus and Giroux, 1977), 6. 


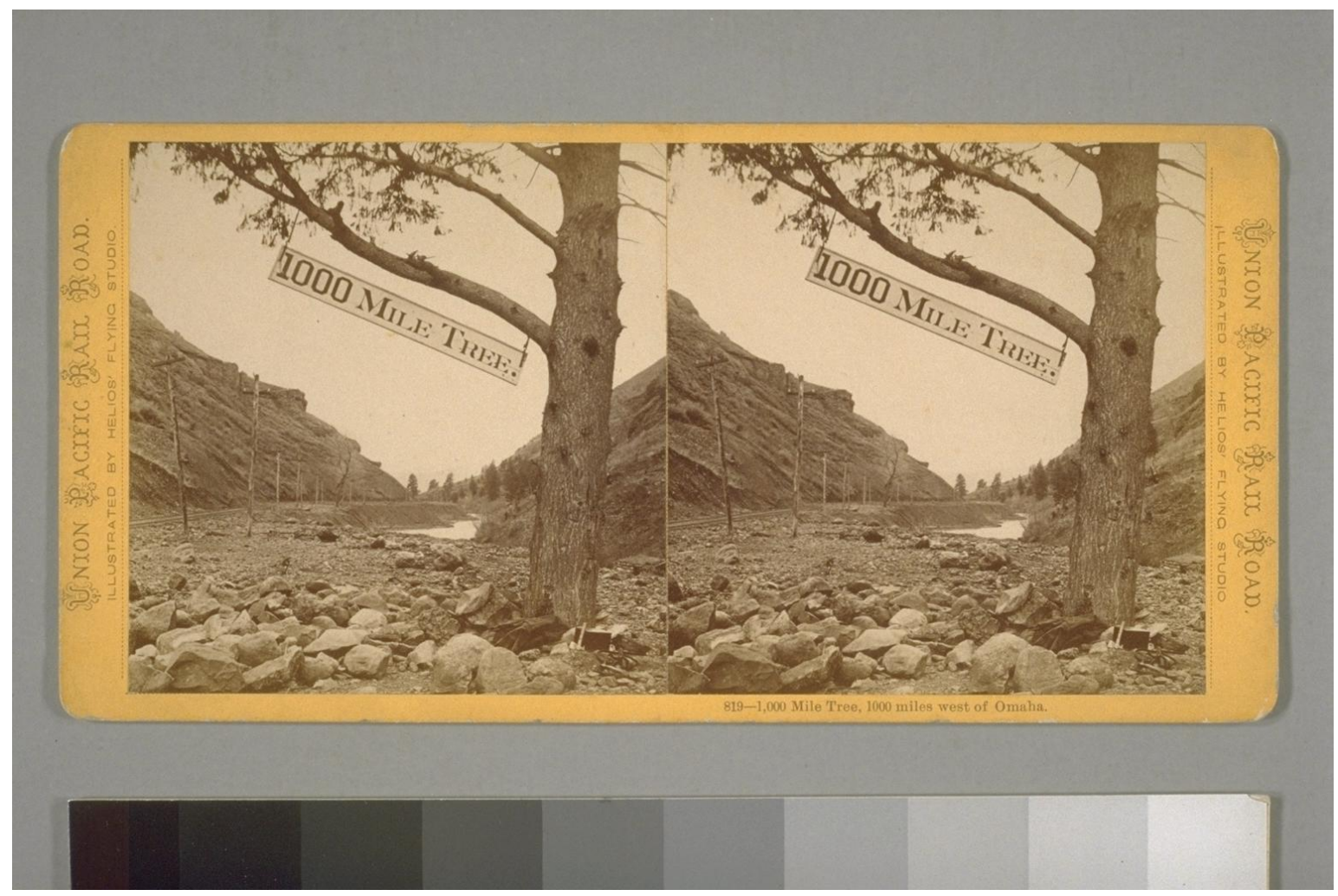

Figure 4. Eadweard Muybridge, Thousand Mile Tree, 1000 Miles West of Omaha. http://www.oac.cdlib.org/ark:/13030/tf8x0nb9c6/?order=1

For Miller, that the tomahawk indicates a "gesturing to American Indian times or that this was once American Indian land." By focusing on a particular detail, Miller lays the framework for his historical argument.

Miller describes the scene in the second photograph (see Figure 5) as barely inhabited, "except for the character who, squinting just barely reveals, is dressed in a long duster and a very wide-brimmed cowboy's hat." 355 Miller reads him as a "sort of hearty Western pioneer."

\footnotetext{
${ }^{355}$ Daegan Miller, "Witness Tree: Landscape and Dissent in the Nineteenth Century United States" (PhD Diss., Cornell University, 2013): 265.
} 


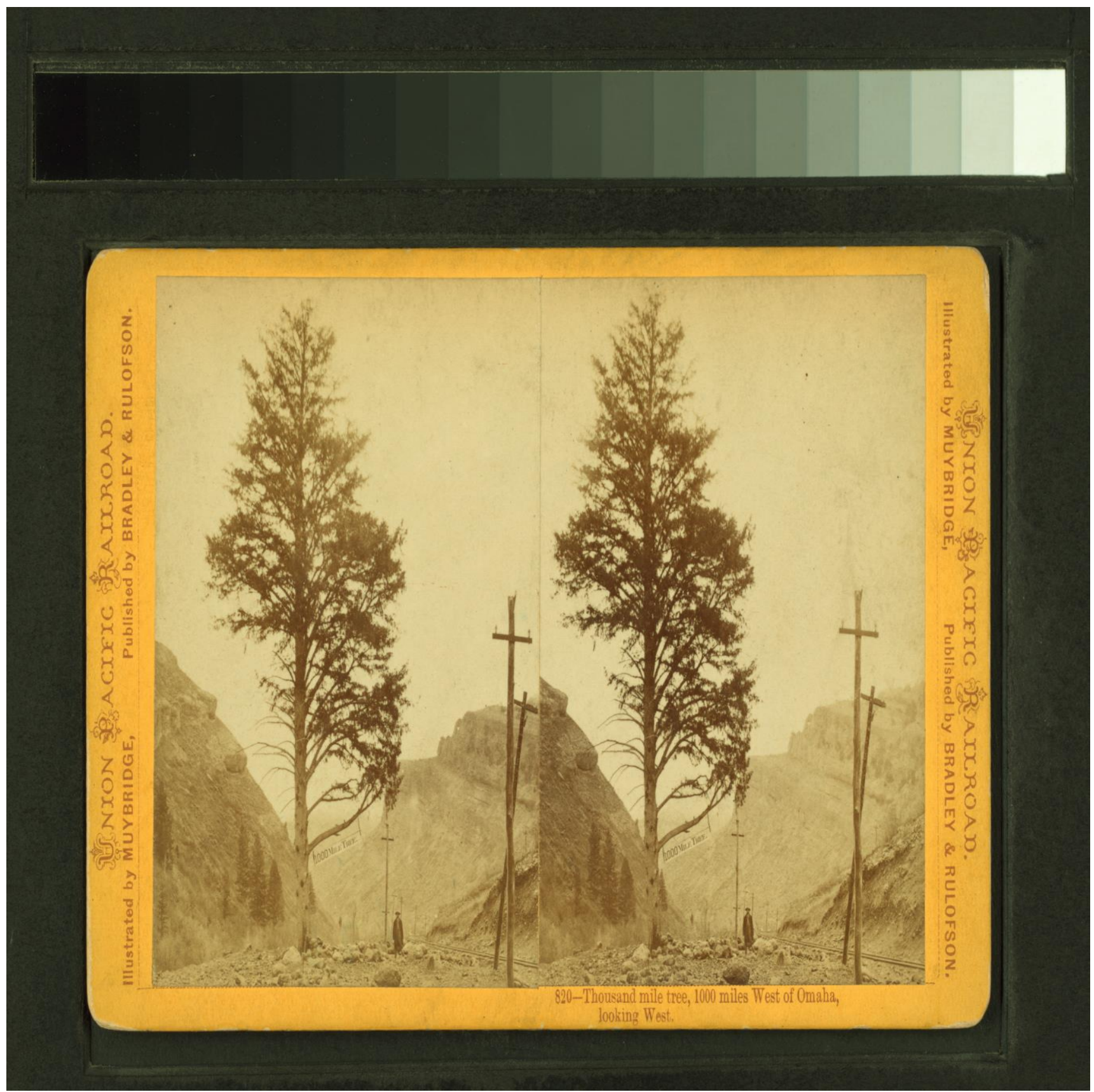

Figure 5. Eadweard Muybridge, Thousand Mile Tree, 1000 Miles West of Omaha, Looking West, http://digitalcollections.nypl.org/items/510d47e0-17c3-a3d9-e040-e00a18064a99

Both photographs, if taken together, suggest "a narrative of conquest, of an absent Indian presence metonymically signaled by that tomahawk succeeded by the view of the western pioneer, hardy and upright, emerging out of the distance to stake his claim." ${ }^{\text {"356 }}$

\footnotetext{
${ }^{356} \mathrm{Ibid}, 266$.
} 
Viewing the third photograph is what motivated Miller to make a deeper historical connection out of this three-part series (see Figure 6). He reads the hunched figure in the photographs as being meant to represent the mythical Wandering Jew, serving as a reminder to viewers about the nature of atrocities and "the unnaturalness of violence."357 For Miller, the depiction of this figure suggests that Muybridge is commenting on "the shade of past injustices that refused to stay dead and buried."

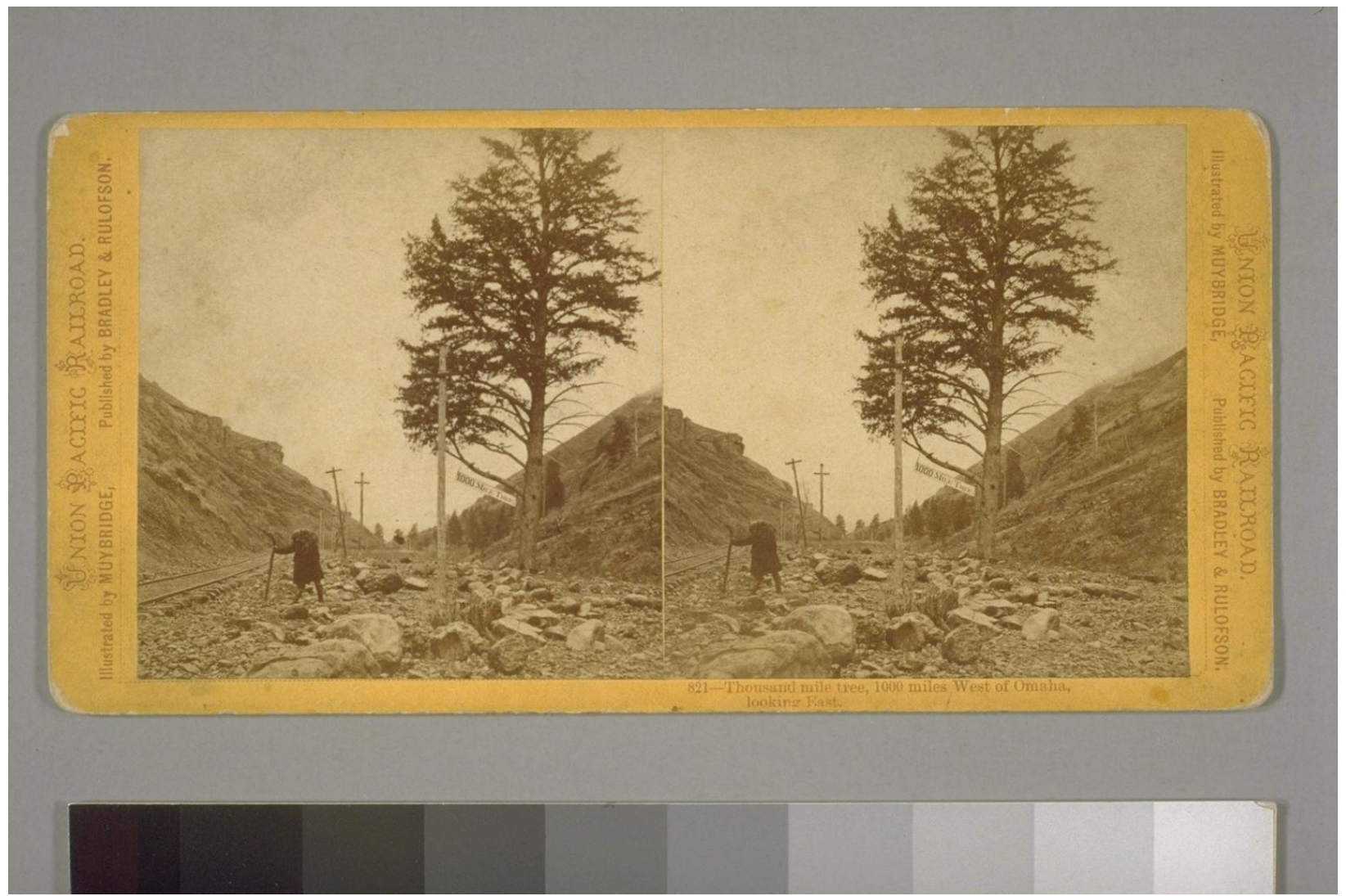

Figure 6. Eadweard Muybridge, Thousand Mile Tree, 1000 Miles West of Omaha, Looking East. https://calisphere.org/item/ark:/13030/tf8v19p556/

In his dissertation, Hilpert explores images of disaster dating from the Civil War to the San Francisco Fire of 1906. Using visual documentation ranging from postcards to photographs, Hilpert argues that images (and image makers) helped shape disaster narratives that reinforced

${ }^{357}$ Ibid, 269. 
American Exceptionalism at the height of industrialization. Hilpert describes the rationale for his image use:

One of the things I was trying to do with this dissertation was get away from the notion that - or at least an alternative view - of these images that came from disasters as just illustration, as they were typically used. You know, they were part of the story of how people understood what took place. And so I wanted to look at them, and even the map that this photograph is pasted to, as kind of a primary source of evidence.

A central event that Hilpert focuses on the Johnstown flood of 1889 . He uses a series of

digitized eight-by-ten photographs taken by E. Walter Histed (see Figure 7) to argue that images of the flood were used to turn the event into a "palatable spectacle" permeated by

"sensationalistic images of struggle and ruin." 358

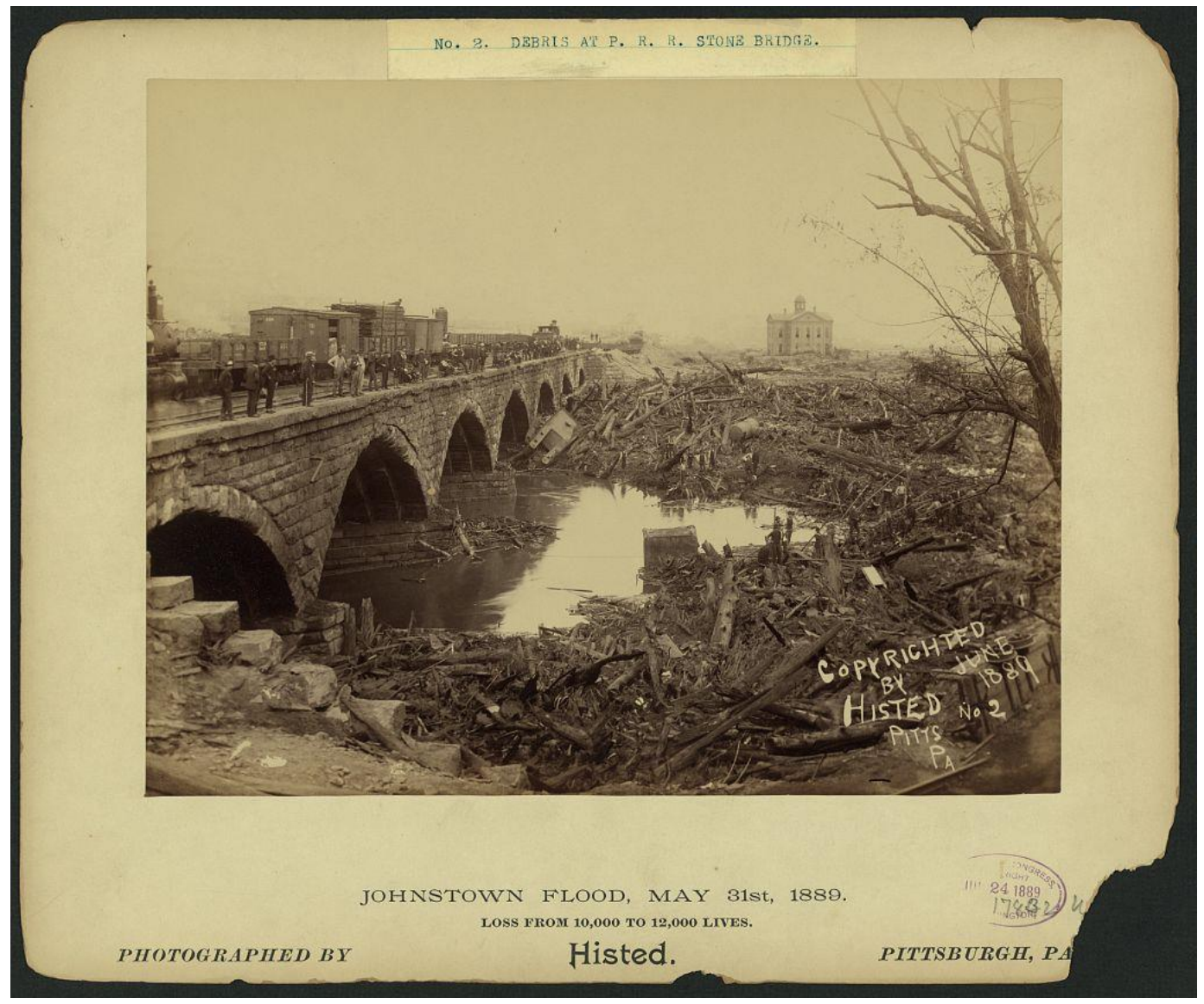

Figure 7. Ernest W. Histed, Debris at P.R.R.Stone Bridge, 1889. http://www.loc.gov/pictures/item/2002707085

\footnotetext{
358 Zachary Hilpert, "Ruins Reframed: The Commodification of American Urban Disasters, 1861-1906," (PhD diss.,
} College of William and Mary, 2014): 110-111. 
Hilpert looks at contextual elements such as photographic captions and related event documentation to structure his argument. For example, he points to the brevity and succinctness of Histed's captions as being indicative of spectacle. "Johnstown Flood, May 31st, 1889. Loss from 10,000 to 12,000 lives. ${ }^{\circledR 359}$ Additionally, Hilpert points out how photographers, journalists, and illustrators were there as soon as the following day to take "pictures like this of scenes that they knew that people were really hungry to see for themselves." For Hilpert, these kinds of images were circulated to fuel "a particular desire on the part of a largely white, native-born consumer class to share in a collective grieving process, one that initially recalled the comforts found in the communal suffering of the Civil War..."360 Hilpert also points to the popularity of so-called "history books" - collections of images with "no real sense of linear narrative" that surfaced usually within days or weeks after disasters. He argues that these kinds of images represent "a product of people's fears of the city in the latter half of the $19^{\text {th }}$ century." They visualize the threats and nightmarish landscape presented by the industrial era.

Sampsell-Willmann's research on the social documentary photographer Lewis Hine suggests that his work represented allegories of his own progressive thinking. Hine is best known for documenting child labor in the first half of the twentieth century; he quit his job as a schoolteacher and became a photographer for the National Child Labor Committee. According to Sampsell-Willmann, part of Hine's motivation for taking photographs of child laborers came from his staunch opposition to their exploitation. She argues that Hine took these photographs to help counter popular misgivings that children were in factories to visit their parents rather than to work. For Sampsell-Willmann, there is a certain degree of intentionality in what Hine chose to

\footnotetext{
${ }^{359}$ Ibid, 20. The actual death toll from the Johnstown flood was 2,209.

${ }^{360}$ Ibid, 1.
} 
capture (and leave out) in framing his images. Hine called his images "Hineographs" and often provided captions and extensive notes to accompany them.

Everything that is in a Hineograph is in there because Hine wanted it, and everything that is missing is absent because Hine left it out. One can, and should, draw conclusions about Hine's intent from the inclusion or exclusion of an adult, a piece of machinery, or a living/working space in a particular image. ${ }^{361}$

Though his work was relatively unknown at the time of his death, Hine is now widely credited as having helped establish standards for workers that effectively cut the number of child laborers in half by 1920 .

Sampsell-Willmann suggests that Hine represented his subjects in a manner that matched his own social conscience. In one of the chapters in her book Lewis Hine as Social Critic, Sampsell-Willmann uses images Hine took of newly arrived immigrants to Ellis Island to make her argument. She focuses on one particular image of a Russian immigrant (see Figure 8).

\footnotetext{
${ }^{361}$ Kate Sampsell-Willman. "Using Lewis Hine's Child Labor Photographs, Part 2: The Miners," The Journal of the Gilded Age and Progressive Era Online http://www.jgape.org/node/123 (2010).
} 


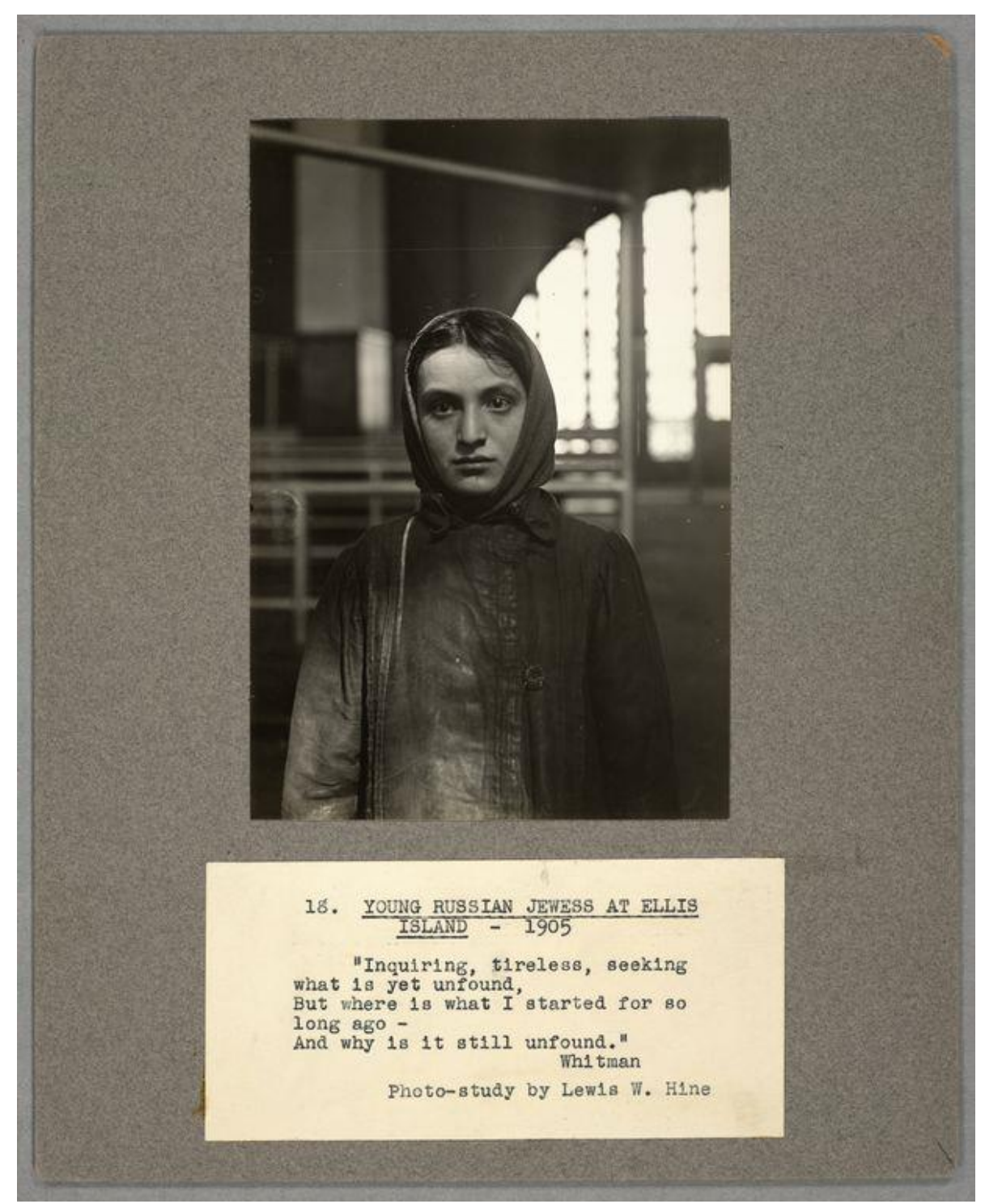

Figure 8. Lewis Hine, Young Russian Jewess at Ellis Island, 1905.

http://digitalcollections.nypl.org/items/510d47d9-4e85-a3d9-e040-e00a18064a99

For Sampsell-Willmann, the anonymous caption is an explicit choice by Hine to identify her as

an immigrant rather than to personalize her. Noting the saturation of anti-immigration sentiment among early $20^{\text {th }}$ century New Yorkers, Sampsell-Willmann argues that Hine purposefully counteracts anti-immigrant assumptions of "othering" in his representation.

By captioning the portrait within a type, Hine addressed the assumptions around him. By allowing her to compose herself for the camera, Hine defeated the "othering" objectivity of social science and offered a new vision of immigration and cultural plurality, one that would become ruling in the 1930s. There the photographer-as-viewer definitely changed the observation. By representing her as a child, tired, yes, but neither powerless nor 
threatening, Hine rebuked the nativists; she was a danger to no one. By photographing her at eye level, looking directly into the camera, she was no longer the cowed refugee; he recognized her individual dignity and strength. By typifying the young woman, Hine acknowledged the current standards of measure used to judge and categorize immigration. Simultaneously he defeated them with his perception of the characteristics that makes each person unique. Perhaps she was not a "typical Russian Jewess" but rather "that particular Russian Jewess." 362

Thus, to make her argument Sampsell-Willmann draws on perceived social norms from the time period to contextualize how Hine's work functions as anti-nativist.

\section{Using photographs as evidence of subject's self-representation}

Another mode of historical evidence employed by historians attributes a certain amount of intentionality to photographic subjects, claiming that subjects consciously represent themselves in a certain manner. In her dissertation examining opera and gender representations in the United States, Kristen Turner uses a carte de visite of the opera singer Minnie Hauk to argue that she purposefully represented herself as "exotic" in publicity images for her performance in the opera Carmen (see Figure 9).

\footnotetext{
${ }^{362}$ Kate Sampsell-Whillmann, Lewis Hine as Social Critic (Jackson: University Press of Mississippi, 2009), 29-30.
} 


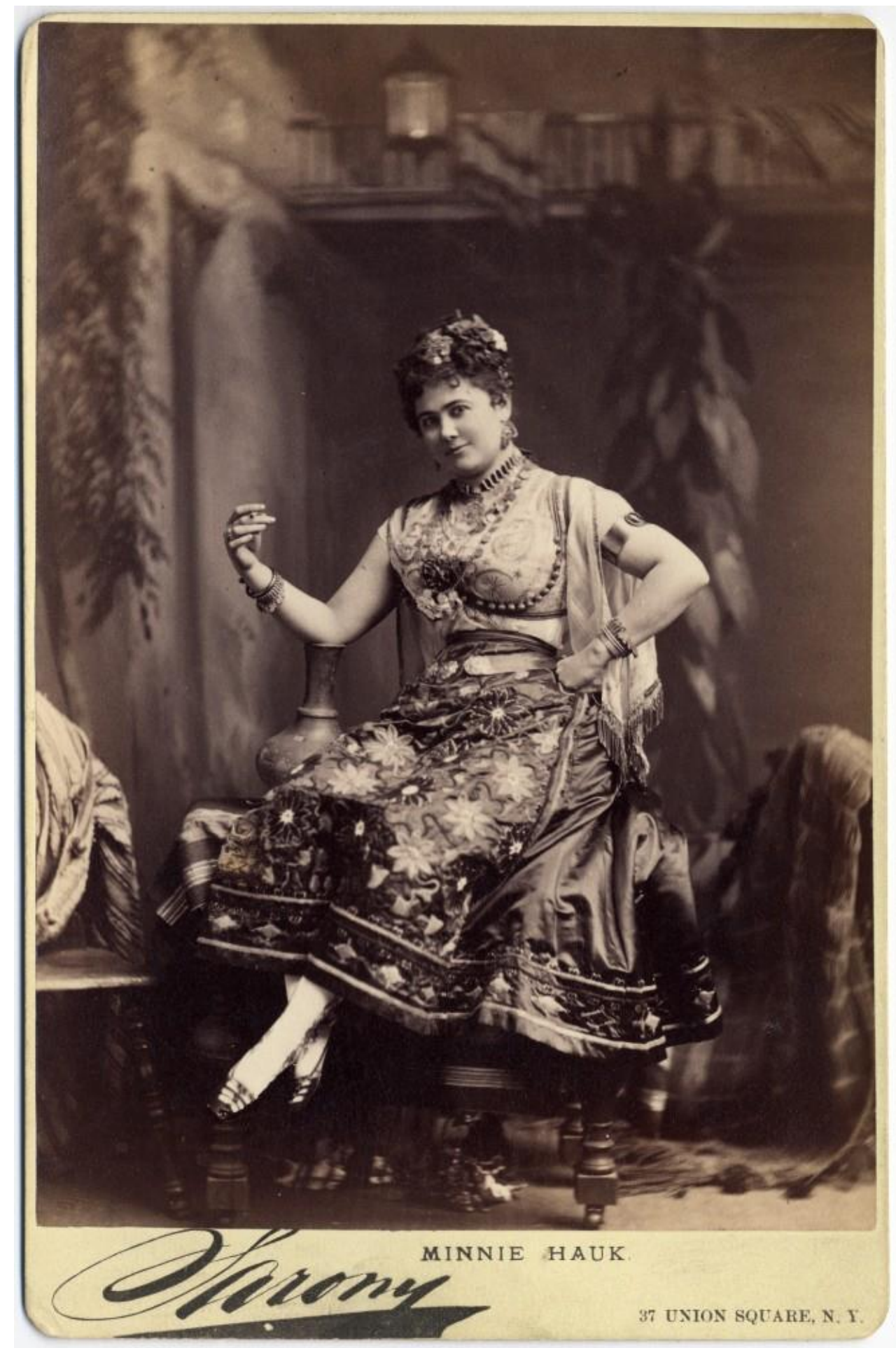

Figure 9. Napoleon Sarony, Minnie Hauk, ca.1880.

https://www.flickr.com/photos/george eastman house/2720790494/

According to Turner, most women participating in American operatic performances during this time period owned their own costumes and were involved in their production. She explains: "I 
know she would have had a lot to do with how that costume looked. And so she wants Carmen to look this way."

Turner points out particular details about Hauk's representation of Carmen that make her portrayal unusual in comparison to how other women have portrayed her. The fact that Hauk is showing her ankles and her bare arms as well as being dressed in elaborate jewelry and embroidery signifies her intention to "exoticize" Carmen "in a sexual manner." In her dissertation, Turner uses quotations from American critical writing at the time period to help situate the particular historical context. For example, according to The North American publication, Hauk's portrayal was "objectionable to the cultivated taste, which looks upon the opera as catering first to refinement, and not at all to realism." 363

Jackson also uses this mode in her research on how communists represented themselves in the media. Her master's thesis examines the social lives and gendered constructions of members of the Workers World Communist Party in California.

${ }^{363}$ Kristen Turner, "Opera in English: Class and culture, 1878-1910” (PhD Diss.), 2015, 367. 


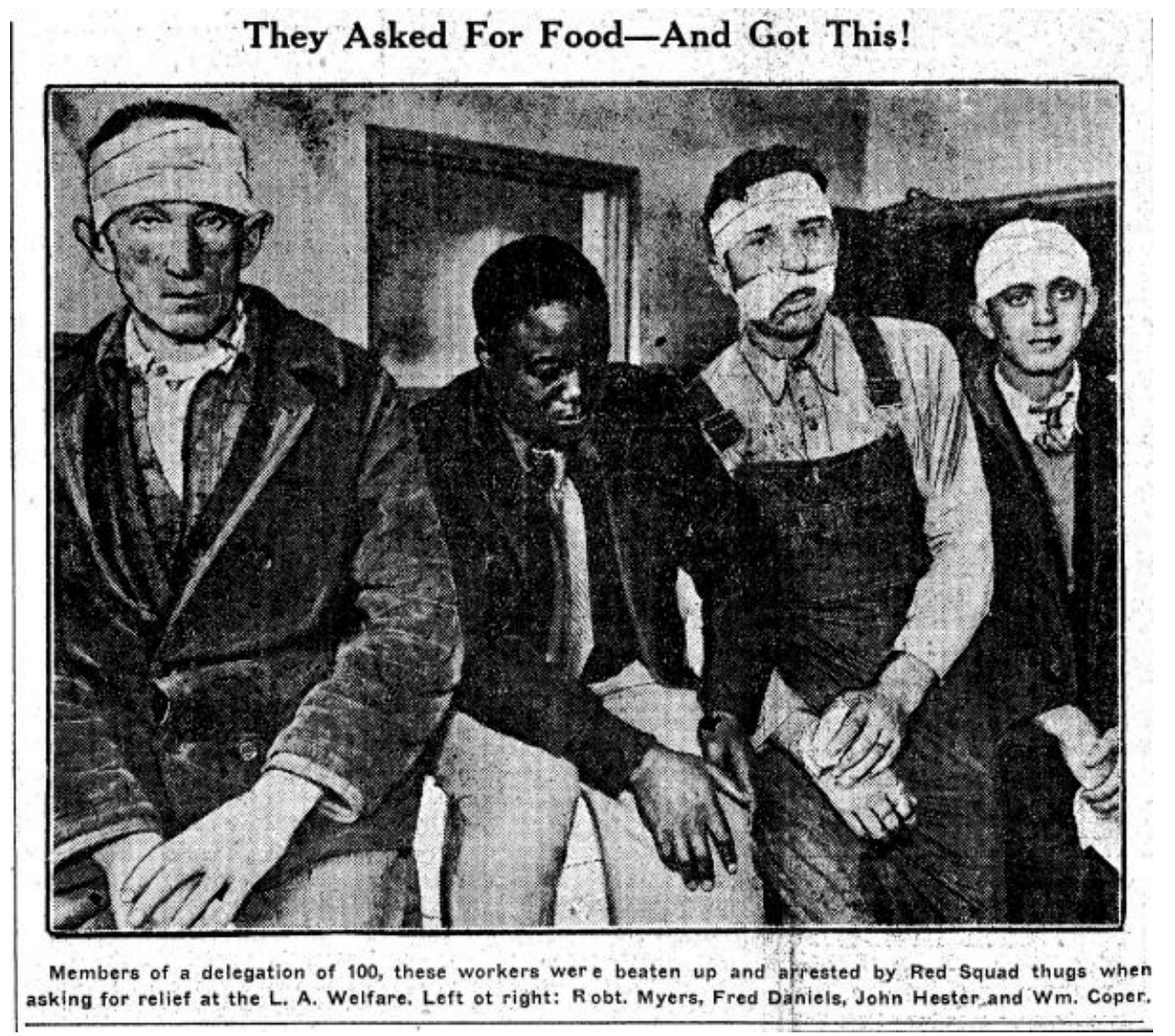

Figure 10. "They Asked for Food and Got This!" Western Worker 2, no.5 (30 January 1933). https://www.marxists.org/history/usa/pubs/westernworker/1933/v2n05-jan-30-1933.pdf

She explains how photographs like Figure 10 transformed the aims of her research.

I really started out to do a social history of the party. I was much more fascinated by their social lives. And then, the more I delved in the photographic evidence...I got fascinated by the way they portrayed themselves in newspapers, and then once I started reading some of the reports about their sicknesses, and you know... once the entire LA staff was either sick or in jail - so there was a lot of discussion about needing to get people there.....and then I started getting interested in this dichotomy of the way they represented themselves in the press, and the way they were actually living. So a lot of it was a combination of a photographic evidence, putting themselves out there as these manly guys who were brawling and getting whipped by the cops, but still out there on the front lines, and the very manly, masculine, muscular images they put in political cartoons. So yeah, it did transform my research from this kind of general "I'm interested in their social life" to "I'm interested in the way that they portray themselves." And they're portraying themselves. 


\section{Using photographs as evidence of the representations of social norms}

In this study, archival photographs are used to demonstrate representations of historical social norms. In his teaching, Miller uses digitized archival photographs in "American History to the Civil War Era" to argue that public perceptions of death changed during the Civil War. In his PowerPoint presentation for his introductory course, Miller juxtaposes contrasting images of death from the time period to make his argument.

Miller references a painting showing George Washington on his death bed surrounded by friends and family in a portrait by Junius Brutus Stearns from 1851. He contrasts this image with a photograph taken by Alexander Gardner in 1863 (see Figure 11).

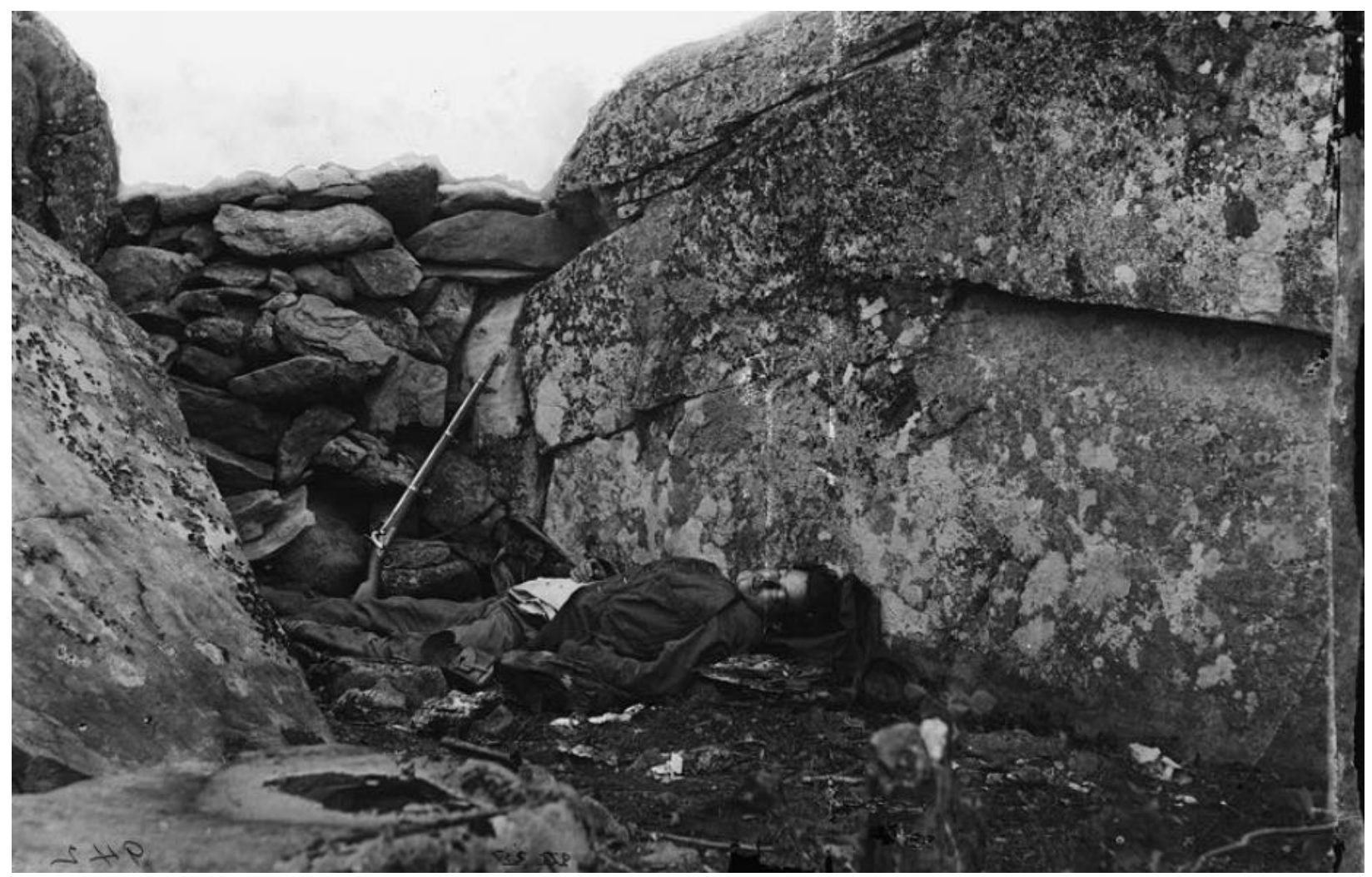

Figure 11. Alexander Gardner, Home of a Rebel Sharpshooter, 1863. https://www.loc.gov/resource/cwpb.04337/ 
Juxtaposing both images together shows how perceptions about the culture of death changed in the space of the Civil War. Miller explains his reading of both images in positioning his argument:

Washington's surrounded by his friends, his family. His wife is in mourning. He's clean, he's ready to meet his maker. He's going to say his last words, they're going to be famous. But once you get to the Civil War. . Y You know, this is an image- if the Washington image is an image of the "good death," the idealized good death- this is an image of a wasteful death, of a bad death. This guy is very clearly not clean, he's not prepared to meet his maker. He's not surrounded by his friends and wife and daughters and all that sort of stuff. And so one of the arguments I try to use this image to make is that what happens when death starts occurring on sort of a mass, industrialized scale that was literally new? At least in terms of warfare, I guess there was the Black Death and things like that, but in terms of warfare in the US. How did it change the way we think about living and dying in and of itself? And so I tried to make this argument about a change in the culture of death, and kind of a fear and something of a nihilism. That death becomes horrible, and something we want to avoid. And it's going to be lonely, it's going to be painful, and the best case scenario is that someone then drags your corpse somewhere and takes a picture of you.

In his teaching about the 1893 World's Columbian Exposition (or World's Fair, as it is commonly known), Hilpert uses photographs as evidence to demonstrate representations of cultural otherness. The focal point of the exhibition was the White City, a nearly 633 acre development which was "intended to display the grandeur of the American urban future."364

For Hilpert, a single photograph (see Figure 12) depicts how foreigners were intentionally positioned on the Midway Plaisance to serve as contrast to potential inhabitants of the White City. ${ }^{365}$ He explains:

Faraway Moses was brought over from Turkey to kind of stand-in for all Turkish people, and be kind of on display on the midway. So you've got the White City on one side, which is the "here's how great everything is in the United States and all the wonderful inventions and innovations that we've accomplished, and all the art we've created, and by the way, everything is white except for one building dedicated to African Americans" and

\footnotetext{
${ }^{364}$ See: Eric Gordon, The Urban Spectator: American Concept Cities from Kodak to Google (Hanover, NH: Dartmouth College Press, 2010): 21-22.

${ }^{365}$ According to Gordon, the Midway Plaisance was "a collection of 'exotic' cultures from around the world' that 'functioned as an important counterpoint to the majesty of the White City." P. 33
} 
then you've got....the midway where you have....all these different nationalities and cultures kind of represented by people that they went and recruited and brought over and set up in kind of like a Disney-fied version of their culture. And so this guy, and I assume the woman standing with him...kind of served as living cultural ambassadors, I suppose. But all of this with the idea that you're supposed to contrast this incredible architectural achievement of the white city with these supposedly kind of primitive-seeming other cultures on the midway. And the way that they've kind of designed the buildings, the way they've laid it out, was "here's where you can kind of go to a zoo of other cultures," in a way. So this image, and a lot of other ones kind of stood in for - I was using them and comparing them with images of the white city.

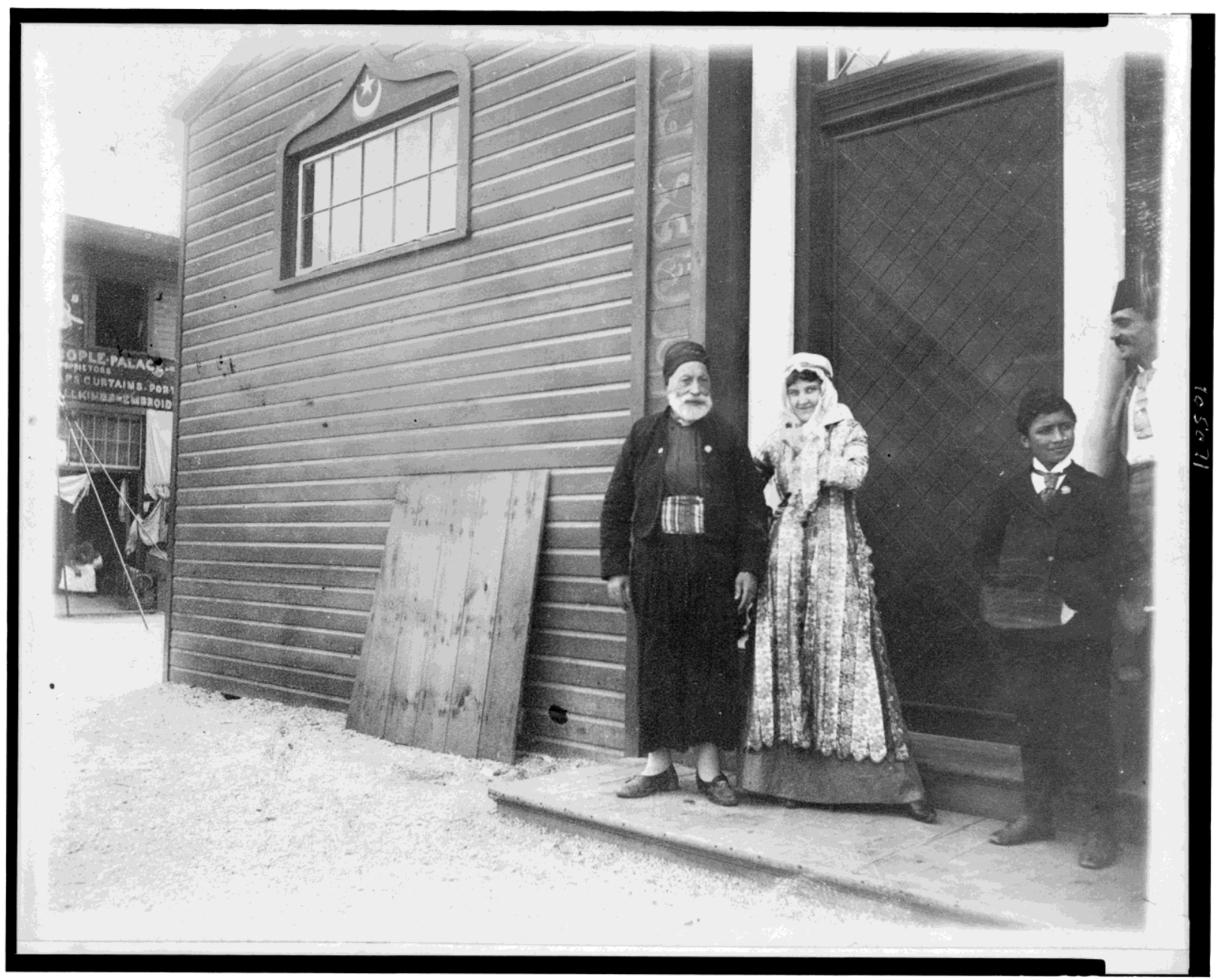

Figure 12. Far Away Moses with woman in Middle Eastern dress at World's Columbian Exposition, Chicago, Illinois, ca.1893. http://www.loc.gov/pictures/item/92505426/

The ability of photographs to signify social norms is also part of their power when used to engage historical thinking. Gold frequently uses photographs as historical documentation in his work on ethnic communities. He uses the photograph below (see Figure 13) to teach his 
students about racism against Japanese Americans during World War Two. Gold explains that the power of the image lies in its ability to engage his audience.

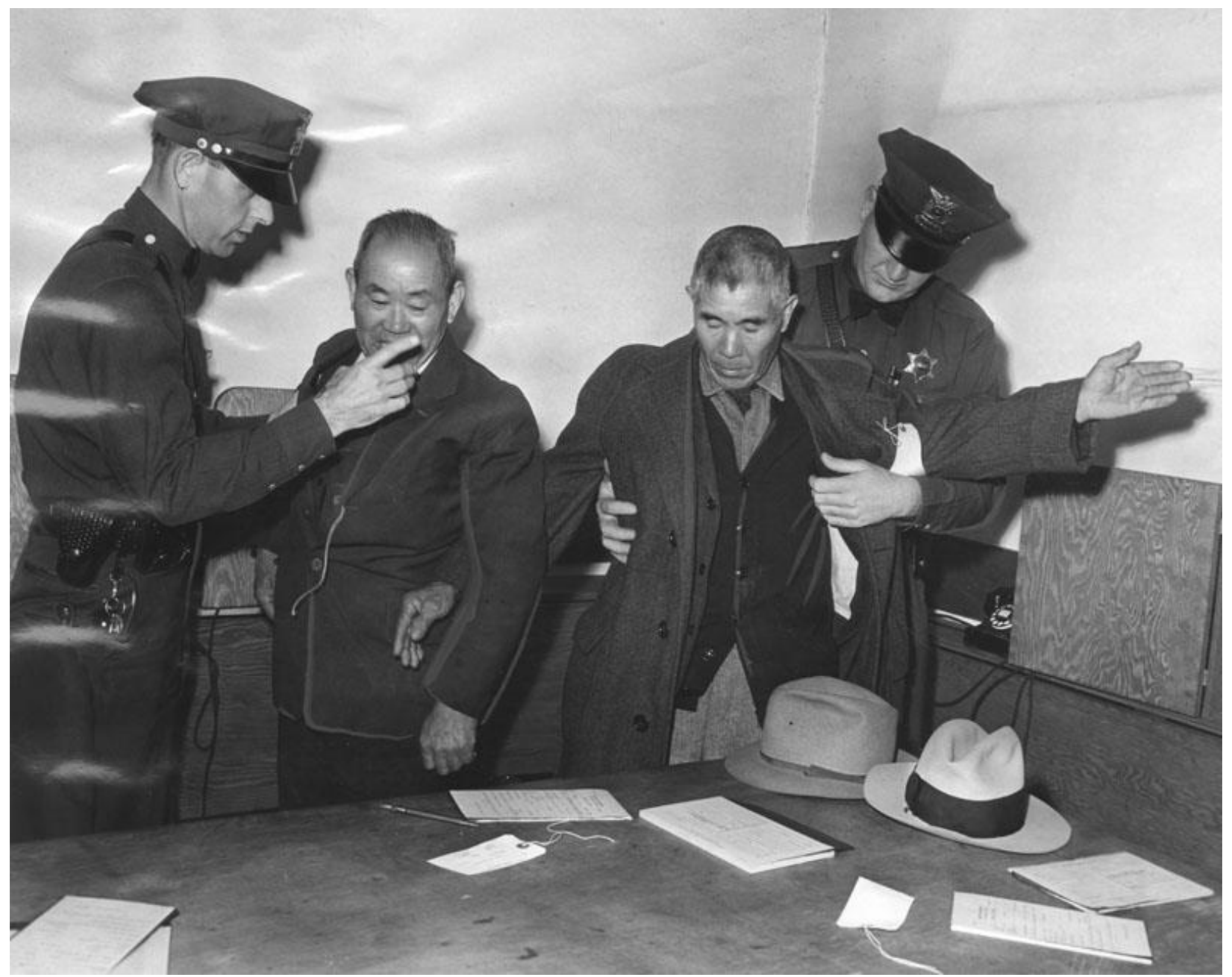

Figure 13. Japanese Captured in Raid, 1942.

http://photos.lapl.org/carlweb/jsp/DoSearch?\&index =tw/\&databaseID=968\&count=10\&tag=245\&terms=Japanese $\%$ 20captured $\% 20$ in $\% 20$ raid

He describes how he interprets this photograph:

You know, as a teaching photo, I think the idea of race and the state being used to bear down on these rather- you know, you feel bad, these little older men being roughed up by these intimidating police- and I think it re-contextualized the idea of Pearl Harbor and the Japanese as being this threatening alien presence and so forth, even though these people were living in the US and they weren't allowed to become citizens. But their kids were, and the kids were interned, too. And just the idea that people who are in business are- if they're not the right kind of people- they're seen as threatening, and society will crack down upon them. And so, you know, racism and the power of the state. And the particular threatening of small business, that it's not so much about war or Pearl Harbor, 
it's about "we don't like these people making a decent living in our country," kind of. They're not one of us.

Nelson uses the photograph below (see Figure 14) teaching his Introduction to American Studies class as an example of race as a social construction.

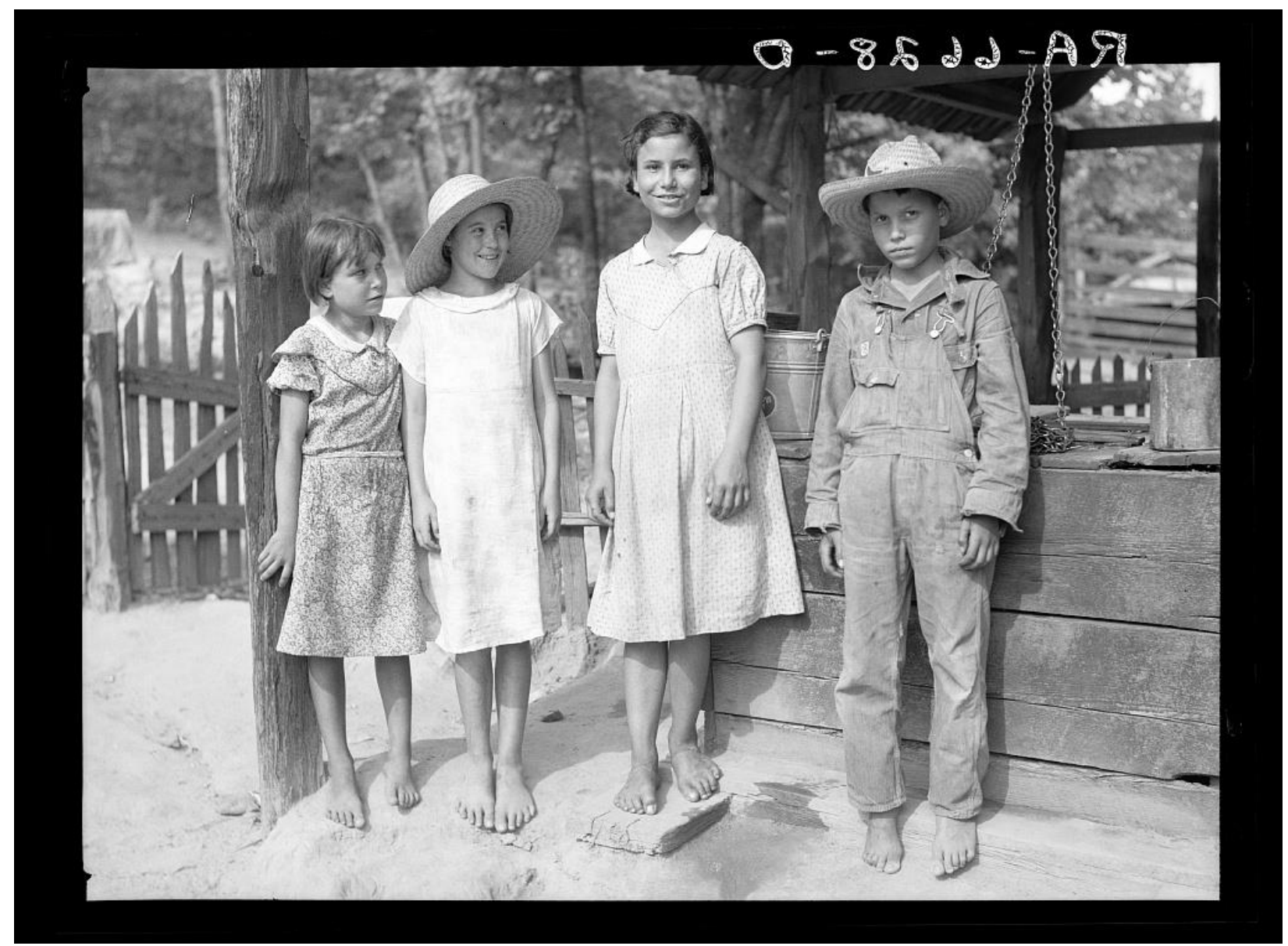

Figure 14. Carl Mydans, "Griffith Children of West Alabama Land Use Demonstration Project," June 1936. http://www.loc.gov/pictures/item/fsa1998020268/PP/

The caption (and full title) of the photograph reads: "GGiffin children' of west Alabama land use demonstration project near Greensboro, Alabama. They are all third or fourth generation resulting from, it is believed, a white woman and a Negro. They are mostly white and refused to be placed with the Negroes, but the whites will not have them. Note carefully the close-up of the four children; all are from the same family and yet differ greatly in appearance." He chose this 
photograph to depict the social construction of race because "it brought to life something that I was teaching in the classroom" in a way that really "drove that home for students."

In addition to serving as historical evidence, photographs can reveal contemporary social norms regarding race, gender, and class. In her instructional work with teachers, Gabriel uses photographs to challenge and/or evoke certain perceptions from her viewing audience. For example, Gabriel uses a digitized photograph that shows the arrest of Civil Rights activist Taylor Washington (see Figure 15).

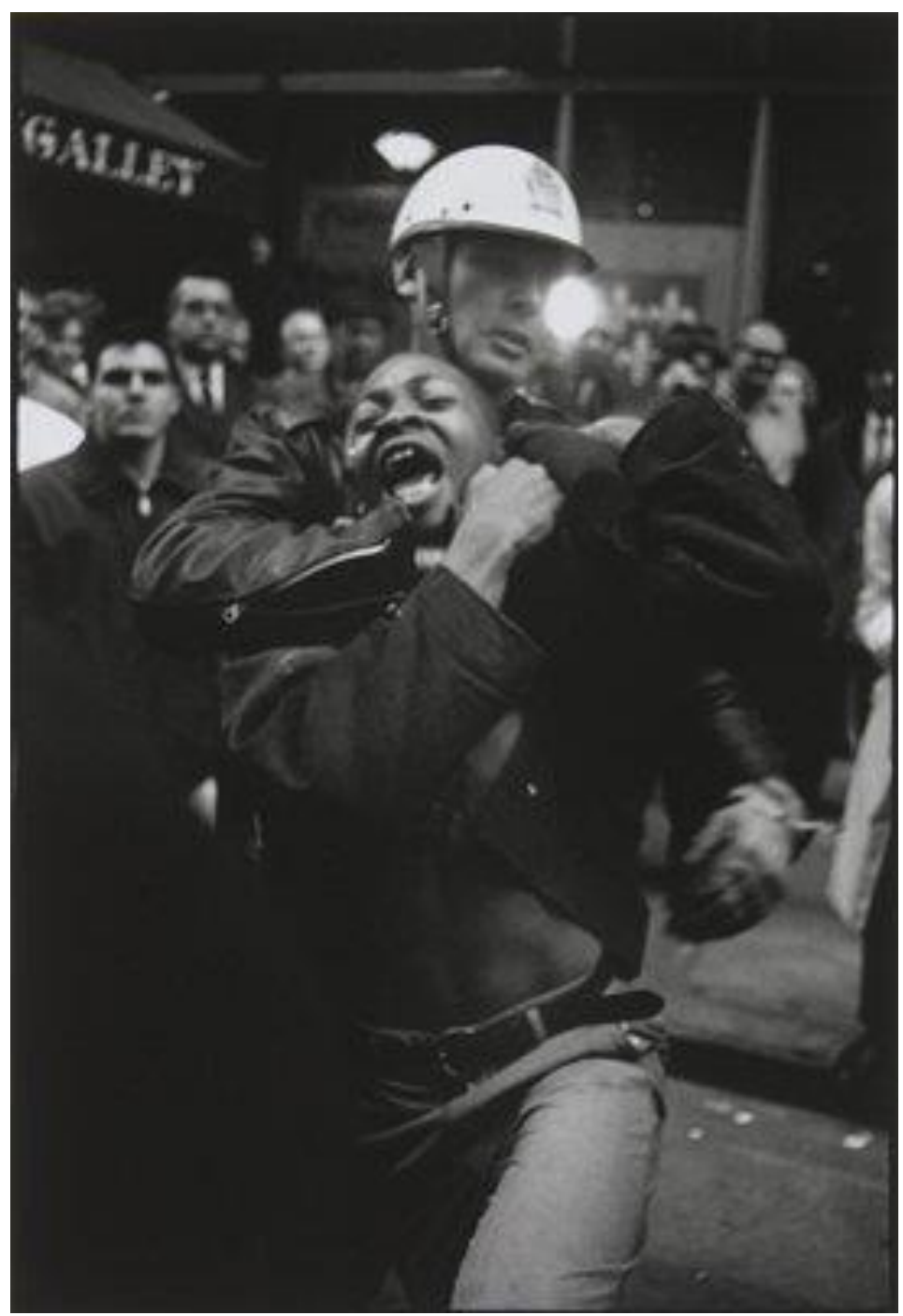

Figure 15. Danny Lyons, Atlanta, Georgia. High school student Taylor Washington is Arrested at Lebs Delicatessen. His eighth arrest, 1963. http://collections.artsmia.org/art/45340. 
Taken in 1963 by photographer Danny Lyons, it was featured at the time on the cover of Pravda $^{366}$ as Cold War propaganda to demonstrate the "lack of democracy and equal participation in American society." Gabriel talks about how she uses the image in her instruction to demonstrate how racist assumptions about African-American men can be triggered in the classroom.

So then I began to sort of try to play with the image a little more, and try to confront this issue of historical consciousness, and ask teachers, "If your students didn't know that this was about the Civil Rights movement, what would they say? If you just showed them this image, with no commentary?" And a lot of the teachers felt that their students would think that he's like, a drug dealer. And so to me, that began to highlight the whole way that black men have been criminalized, and that we don't allow them to have other roles in society.

Gabriel's use of photographs shows their ability to serve in dual capacities; as a source of historical documentation and as the site of projected interpretation.

Black uses visual materials such as maps, photographs, paintings, and advertisements in her freshman writing seminar on American Visual Culture "as a window to understanding historical events, places, and people." In a similar manner to Gabriel, Black tries to provoke contemporary assumptions using historical photographs. In a power-point presentation for a class lecture, Black juxtaposes several photographs taken by the female photographer Francis Benjamin Johnston, who originally documented solider life aboard the USS Olympia during the Spanish-American War (see Figure 16)

\footnotetext{
${ }^{366}$ Pravda is a magazine made in the former Soviet Union.
} 


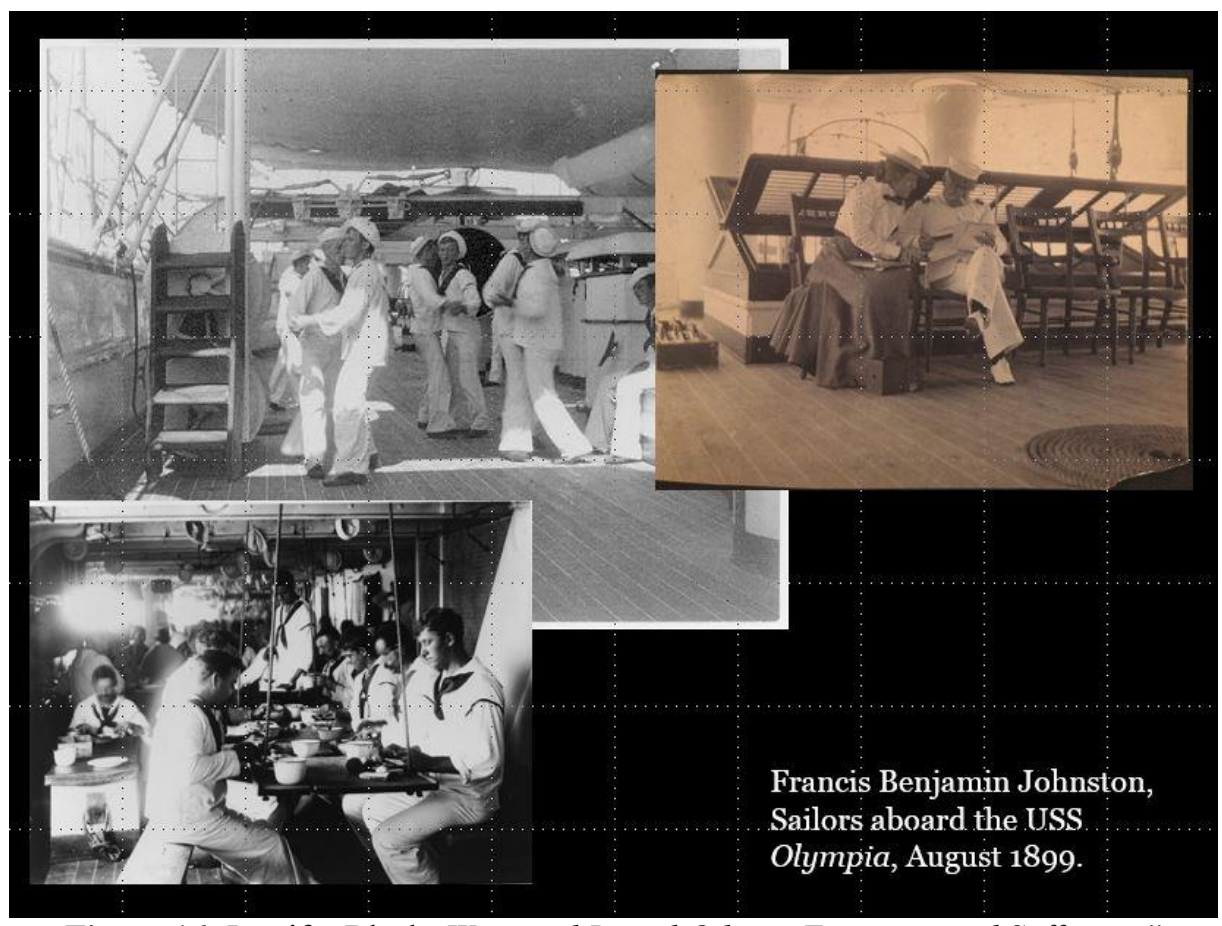

Figure 16. Jennifer Black, Wars and Racial Others: Feminism and Suffering," Presentation, Misericordia University, 2014.

Following the argument of historian Laura Wexler in her book Tender Violence, Black suggests that the presence of a woman aboard the ship helped to pacify and counteract circulating rumors that American sailors had raped and killed civilians in Manilla. According to Wexler's argument, photographs capturing activities like soldiers practicing ballroom dancing with each other (see Figure 17) helped to paint a very domestic vision of the military. 


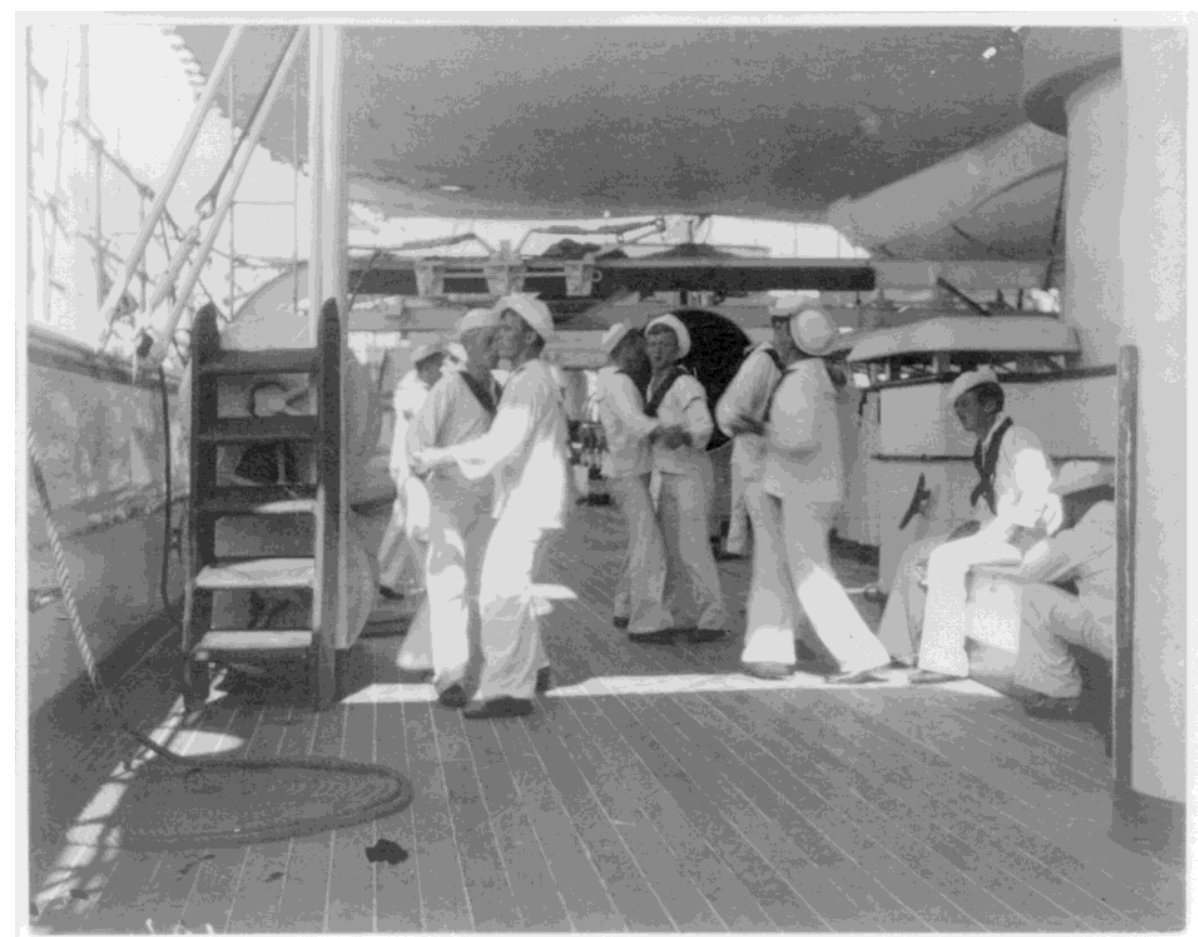

Figure 17. Francis Benjamin Johnston, Sailors Dancing with Each Other Aboard the USS Olympia, 1899. http://www.loc.gov/pictures/resource/cph.3a47711/?co=fbj

Black recounts Wexler's argument in explaining that these photographs were released at "a very specific time when rumors about these atrocities are starting to surface in the American press.” However, in her seminar, Black uses Johnston's photographs primarily to provoke her students to make assumptions about the soldiers during their viewing experience. She explains:

So we've already had this discussion about what is proper, middle-class activity for white men, and what is proper middle-class activity for white women, at this point. And the students appreciate seeing what they think is an image of homosexuality....and we have a whole discussion about how our $21^{\text {st }}$ century perspective is inadequate for understanding what's happening in this image and how we need to put ourselves in the context of the $19^{\text {th }}$ century to really appreciate and understand the impact that this image would have had. Because from a $21^{\text {st }}$ century perspective, there's "weird gay sailors who are dancing together."

Krase is an active photographer who uses his own images to counter stereotypical assumptions about specific communities, particularly ethnic enclaves. He maintains an online photographic archive featuring digitized images he took of "ethnic neighborhoods" all over the 
world. ${ }^{367}$ He foregrounds his analysis in the notion that place (and our interpretations of it) figures prominently into our daily experiences.

In his book Seeing Cities Change, Krase uses photographs to explore how urban vernacular landscapes are constructed and consumed. He analyzes photographs from sixteen Chinatowns across the world to identify common visual features of what he terms "idealized ethnic urban spaces." 368 Krase discusses the ways in which inhabitants of Chinatown "inscribe their neighborhood spaces" as an important component of agency. He explains:

One way by which Chinese assert themselves is through artistic and cultural activities. Sometimes they take place inside buildings and other structures such as cultural centers, schools, theaters, art galleries, and museums. In other cases they take place in plain view on the streets, in front yards, and on the walls of the neighborhood. ${ }^{369}$

Krase uses different images (such as Figure 18) to demonstrate the latter case. In his presentation, he juxtaposes this image with other photographs of Chinatowns in other cities from 2001-2007.

\footnotetext{
${ }^{367}$ Jerome Krase and Timothy Shortell, Gallery. http://www.brooklynsoc.org/blog/gallery3/

${ }^{368}$ Jerome Krase, Seeing Cities Change: Local Culture and Class (Farnham: Ashgate, 2012): 99.

${ }^{369}$ Ibid, 100.
} 


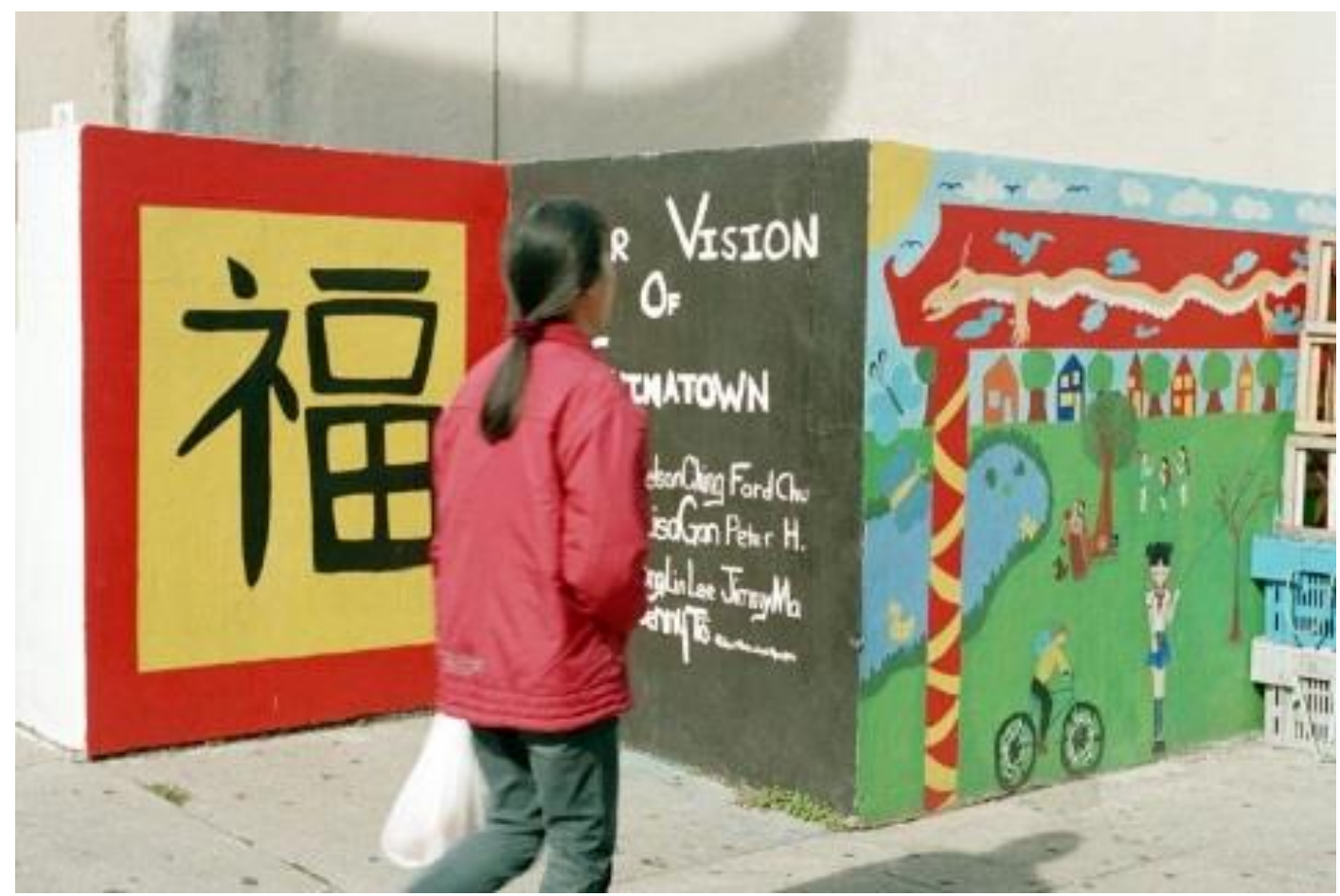

Figure 18. Jerome Krase, Our Vision of Chinatown Mural, Philadelphia, 2005. http://www.brooklynsoc.org/blog/gallery3/index.php/krase012/06476514

\section{Historians use photographs as evidence of historical documentation.}

A common mode of historical evidence is the use of digitized photographs as historical documentation, or evidence of "the way things were." The testamentary power of the photograph has been written about poignantly by Roland Barthes. He explains, "The photograph does not necessarily say what is no longer, but only and for certain what has been." ${ }^{370}$ As spatiotemporal bounded forms of documentation, historical photographs can help verify, disprove or clarify particular details for historians. For example, Grunert uses photographs from the turn of the century to examine the different methodologies taken by two taxidermists, Carl Akeley and William Hornaday. Although both men had similar training and apprenticeships, they used quite different techniques in their practice. Gruner describes how in one photograph

\footnotetext{
${ }^{370}$ Roland Barthes, Camera Lucida, New York: Hill and Wang (1981): 85.
} 
(see Figure 19), Carl Akeley is "sculpting the elephant. He's got clay on top of the frame, and he's sculpting it to make the ridges and the muscles, and to make the folds in the skin."

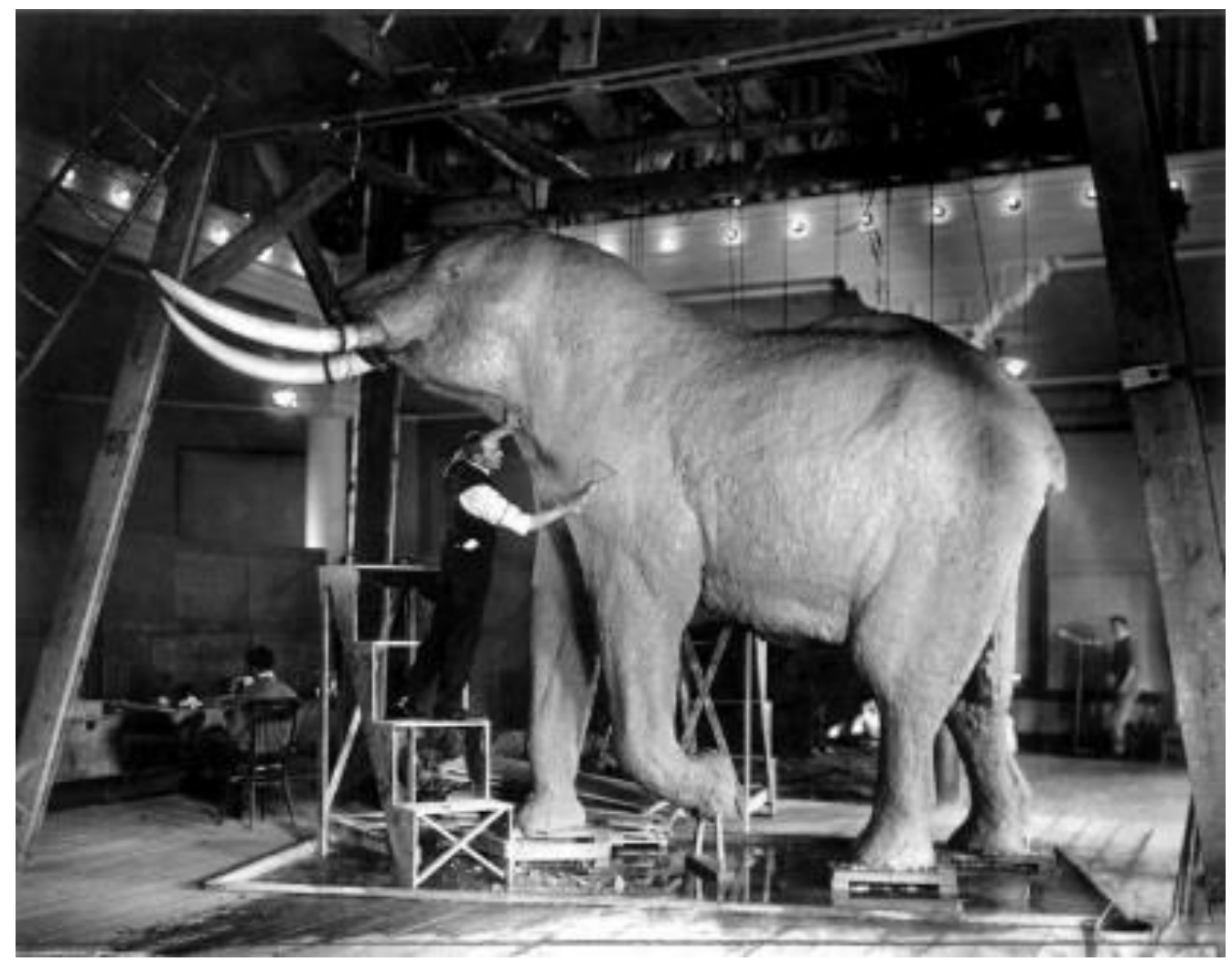

Figure 19. Carl Akeley creating model of Bull Elephant for Elephant Group,1914. http://images.library.amnh.org/photos/ptm/catalog/desc/179522/

However, Grunert points to another photograph (see Figure 20) that shows how Hornaday is

"using string to wrap around the tiger." Grunert was able to use taxidermy manuals from the time period in addition to these digitized photographs to get a sense of what "contemporary" methodologies looked like. 


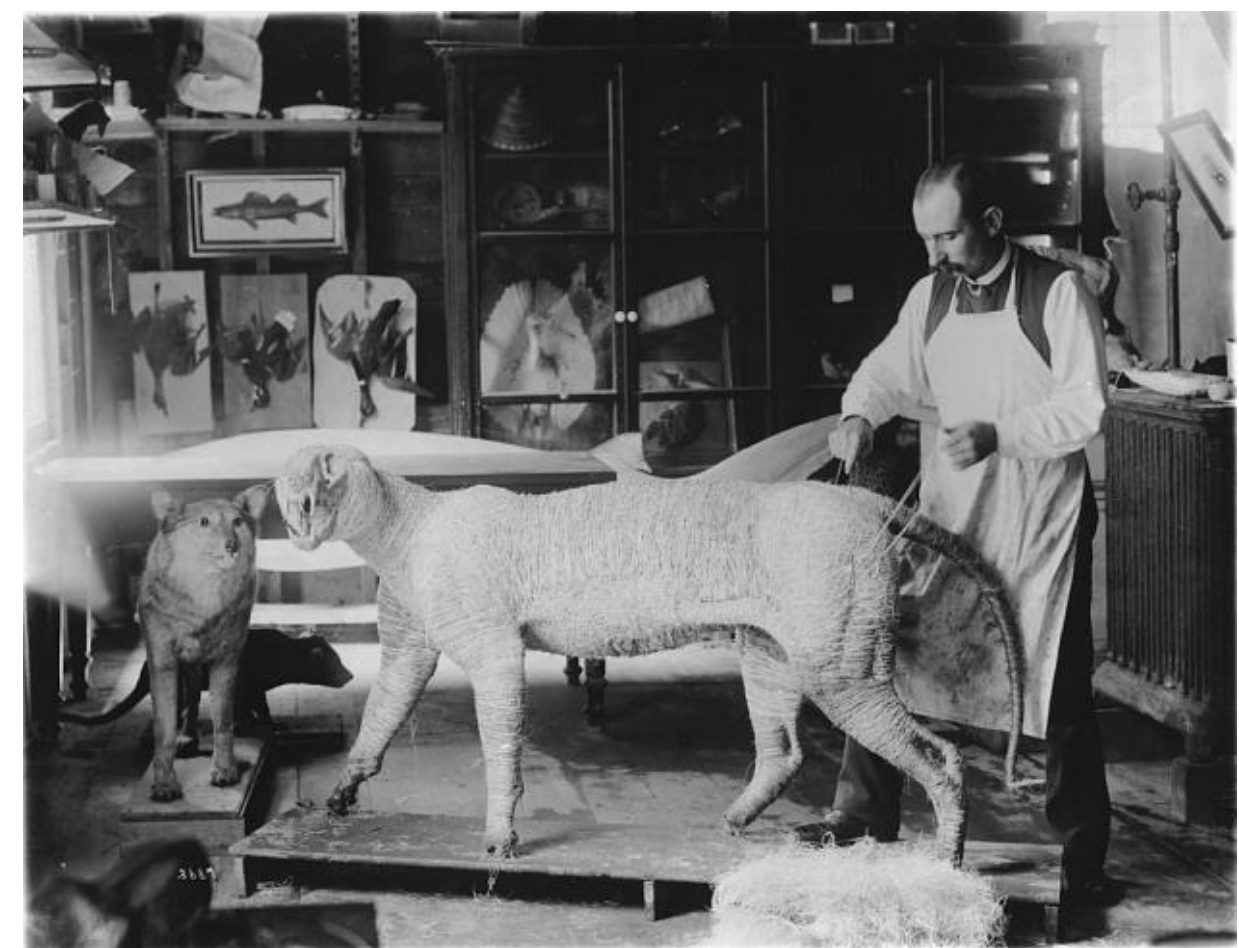

Figure 20. William Temple Hornaday, ca.1880. http://siarchives.si.edu/collections/siris_sic_9666

While juxtaposition of images can be helpful for showing contrasting types of historical documentation, it can also be a useful strategy for uncovering similarities. For Nelson, the frequency with which he came across posed photographs (see Figure 21) helped to fuel his larger argument about photographic and musical expression in early $20^{\text {th }}$ century African-American communities.

But to really bring home the points that I was making, I started bringing in other images that were taking elsewhere. Otherwise, I would be portraying this as something that was just happening in Alabama, which of course it wasn't. And so my use of digital images really comes into play- Well, I have two thoughts on that. One is looking for similar pictures across the United States, which I wouldn't have been able to do without being able to access digital archives. And this is probably useful, although it's pretty lame and boring, but I did a lot of Google image searches. And spent a lot of time sort of honing how I did that and to come up with a lot of images like the one I sent you of those musicians in Kansas. 


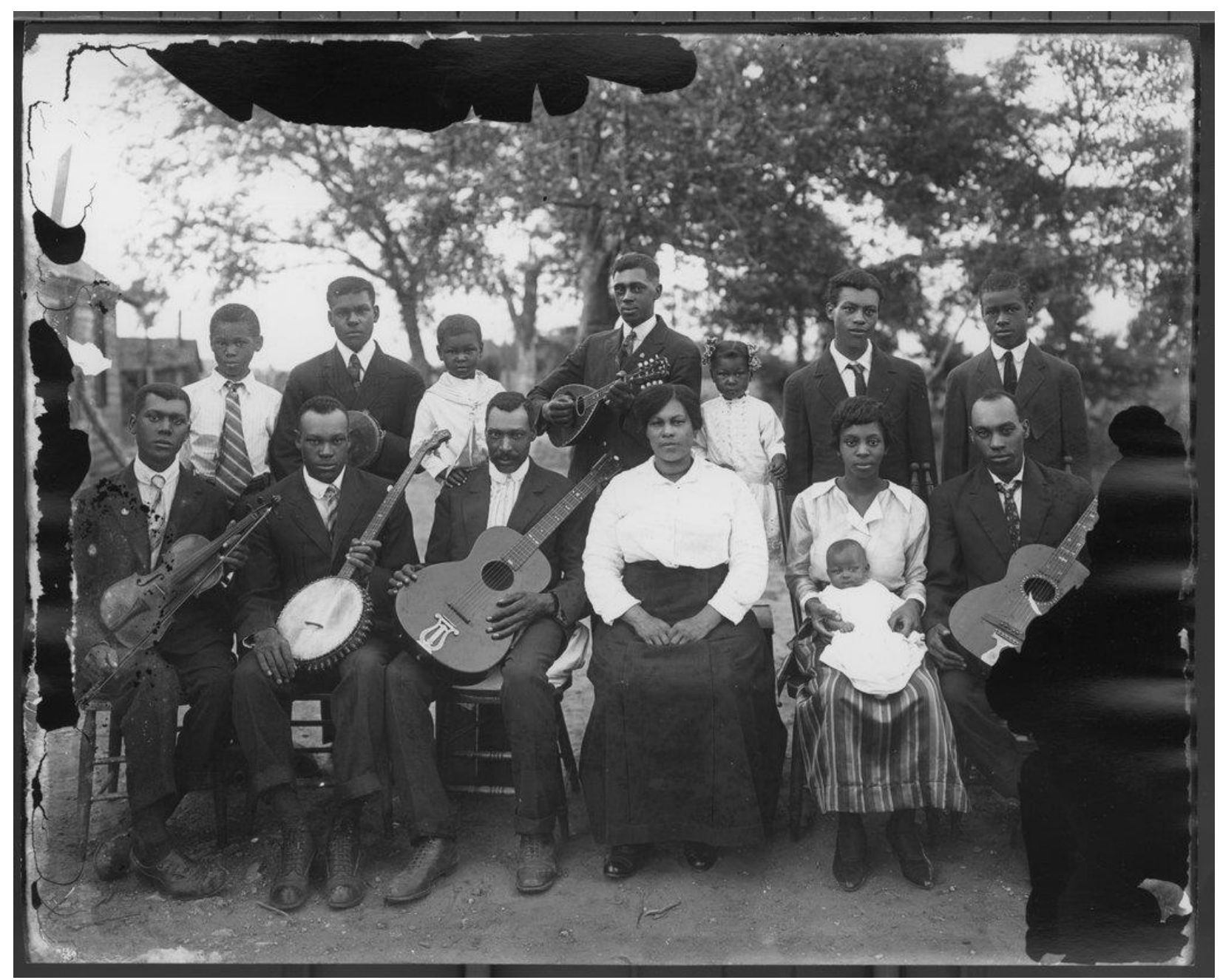

Figure 21. Alfred Lawrence, African-American Musicians, ca.1890-1912. http://www.kansasmemory.org/item/220205

Though his research focused on one particular rural community in Alabama, Nelson uses photographs like this in concert with other images from across the United States to argue that posing with musical instruments was a common expressional practice in American community life. He explains:

So, in this case, I see these musical instruments in the photograph as showing the understanding of American culture, writ large, and also because it's truly a family portrait, as expressions of domesticity and family that are in a lot ways brought to life by the appearance of these instruments that placed such a vital role in middle class American culture. Plus it's also very much related to music and the fact that this is the time when music was made in the home. More than ever. So with these musical instruments, you're really bringing your home outside and making it a part of this picture. 
Nelson directly credits digital archives as being responsible for his ability to establish the commonality of such expressions across the United States.

Clark uses digitized photographs as evidence of "commonplace perspectives" during the Civil War. She teaches a lesson unit entitled "Civil War Expectations" which depicts scenes from a union camp during the Civil War (see Figure 22).

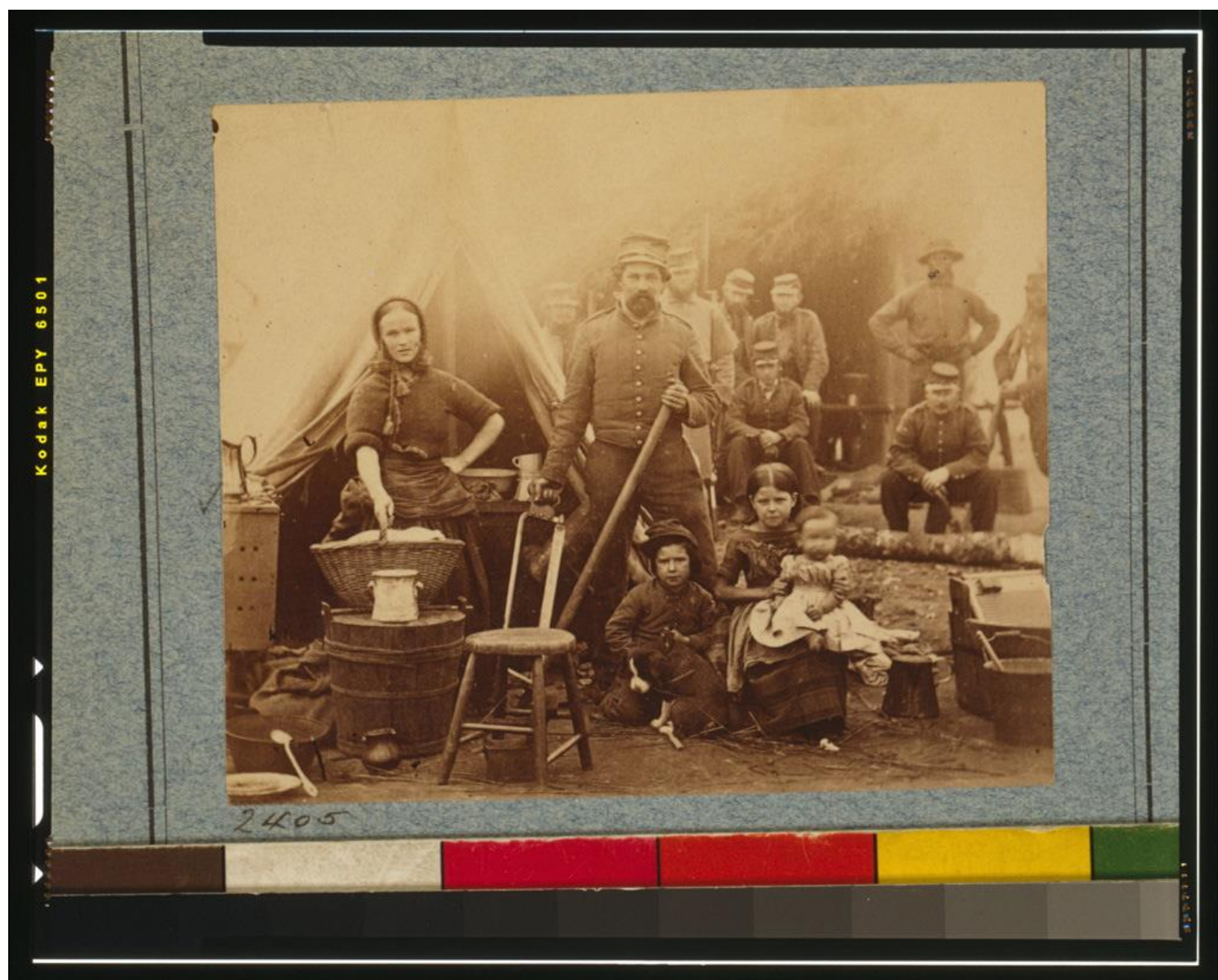

Figure 22. Camp of 31st Pennsylvania Infantry near Washington, D.C., 1862. http://www.loc.gov/pictures/item/98507952

Based on specific details in the photograph, she argues it represents a typical snapshot of everyday life during the Civil War.

And it's such an intriguing image, to me. Particularly because it says a lot about war and it says an awful lot about who was present during the Civil War, on the battlefront. We 
see these children, and we see women, you know, this women laundress... and the soldiers, just this staged photograph says a lot about what it was like on the front, and that's certainly something I want students to understand. The war is not just about soldiers, it's about more than that.

Clark aims to incorporate images into much of her teaching. In her classes, she often assigns homework that asks students to analyze images using the photographic analysis worksheet from the National Archives. ${ }^{371}$ According to Clark, photographs such as Figure 22 are especially helpful for helping to characterize the artefactual qualities of physical photographs. She explains that students are "not used to thinking of a photograph as a physical thing. And what I like is this looks like a physical object. You can see the Kodak on the margins, and its catty-corners, and you see the little color-bands on the bottom."

Material aspects can contribute in different ways to historians' abilities to make historical claims. Examining photographs as physical objects can prompt serendipitous discoveries that might not be discernible in their digital counterpart. For example, it wasn't until Miller used a magnifying glass on a photograph (see Figure 23) that he discovered critical details in the image that helped formulate his research argument.

\footnotetext{
${ }^{371}$ Photo Analysis Worksheet. National Archives Website. Retrieved from: http://www.archives.gov/education/lessons/worksheets/photo.html
} 


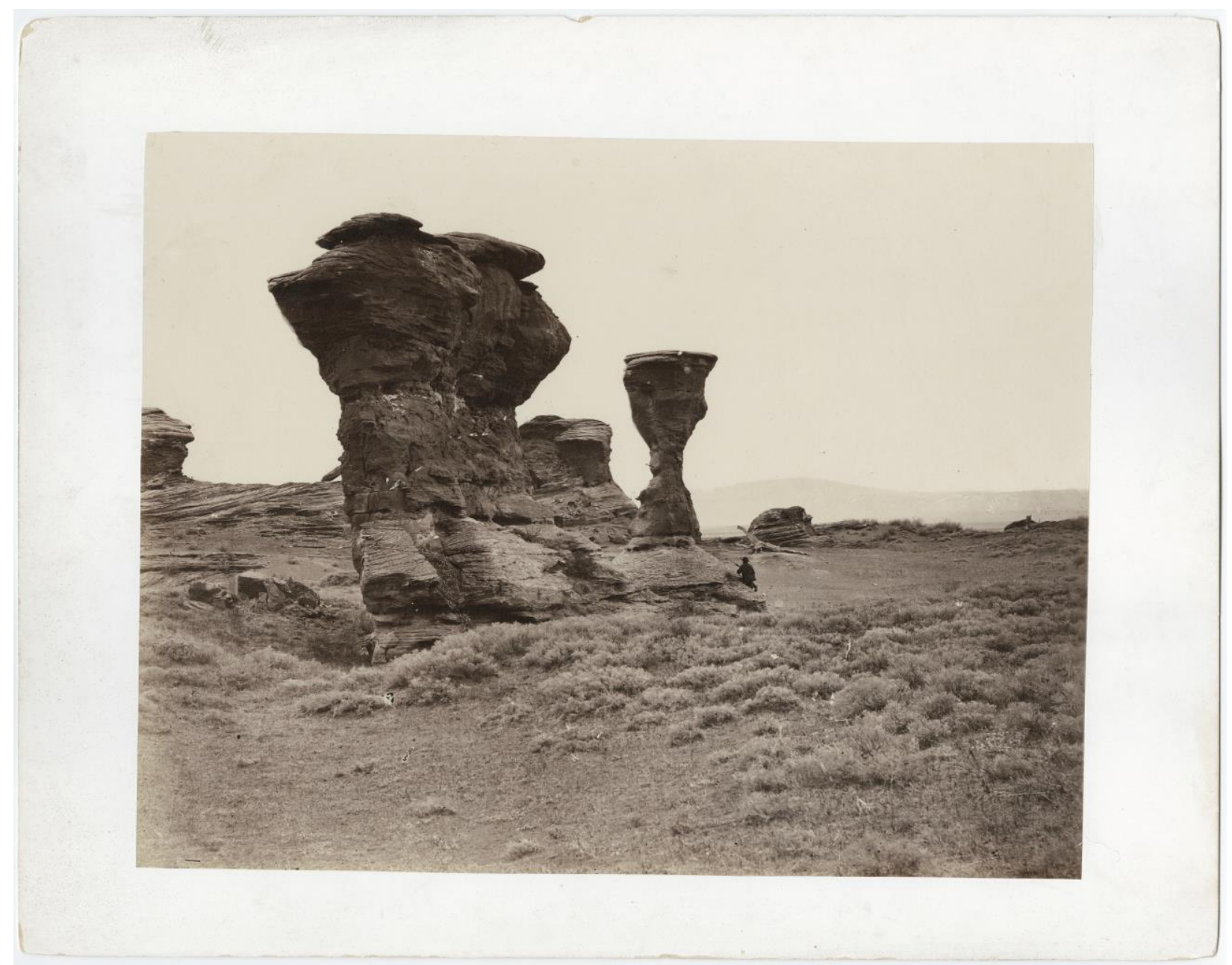

Figure 23. A.J. Russell, Vase or Dial Rocks, Red Buttes, ca.1864-1869. $\underline{\text { http://brbl-dl.library.yale.edu/vufind/Record/3529455 }}$

He explains:

So, there are a number of things that I never would have seen, I think....you know, the physical image has a whole affect to it that you don't get from a glowing screen, I think. Especially these $19^{\text {th }}$ century glass plate collodion-albumen prints. When I got to the Beinecke, I was looking at an AJ Russell image called "Dial Rock" or "At the Foot of Dial Rock" and it's this weird photo where in the center of the frame there's this weird like, eroded sandstone monolith kind of deal. And there's a guy on the right hand of the monolith with his back to the audience, and he's got a rifle, but he mimics the sort of cocking and aiming. And it's a weird image, it's a tense image, and all the sort of drama happens on the right hand of the frame, where the guy is looking off-frame with his gun, getting ready to shoot. But on the left hand side of the frame, sort of hidden in the shadows - I only actually saw this when I got one of those magnifying loops to look really deeply - there's a human ribcage and bleached pelvis, sort of, on the left hand side. And it sounds like a really small detail, but actually - when you start to dig into the scholarship about what $19^{\text {th }}$ century landscape photographers were doing - that detail of the guy who looks like he's about to fight for his life, and on the other side of the frame is 
a bleached human skeleton, it actually becomes a really significant detail that can help change the story that we tell about $19^{\text {th }}$ century landscape photographers. And I just wouldn't have seen it - I've seen it, now that I know it's there, I can look at the digital image from the Beinecke, and zoom and zoom in to see it, but I never would have seen it if I was just looking at a 2inch by 3inch digital version.

In contrast, digitized photographs can provide clarity that might not have been discernible in the print version of a photograph. For example, Roach explains how a high quality version of one particular photograph (see Figure 24) changed and enhanced his historical argument.

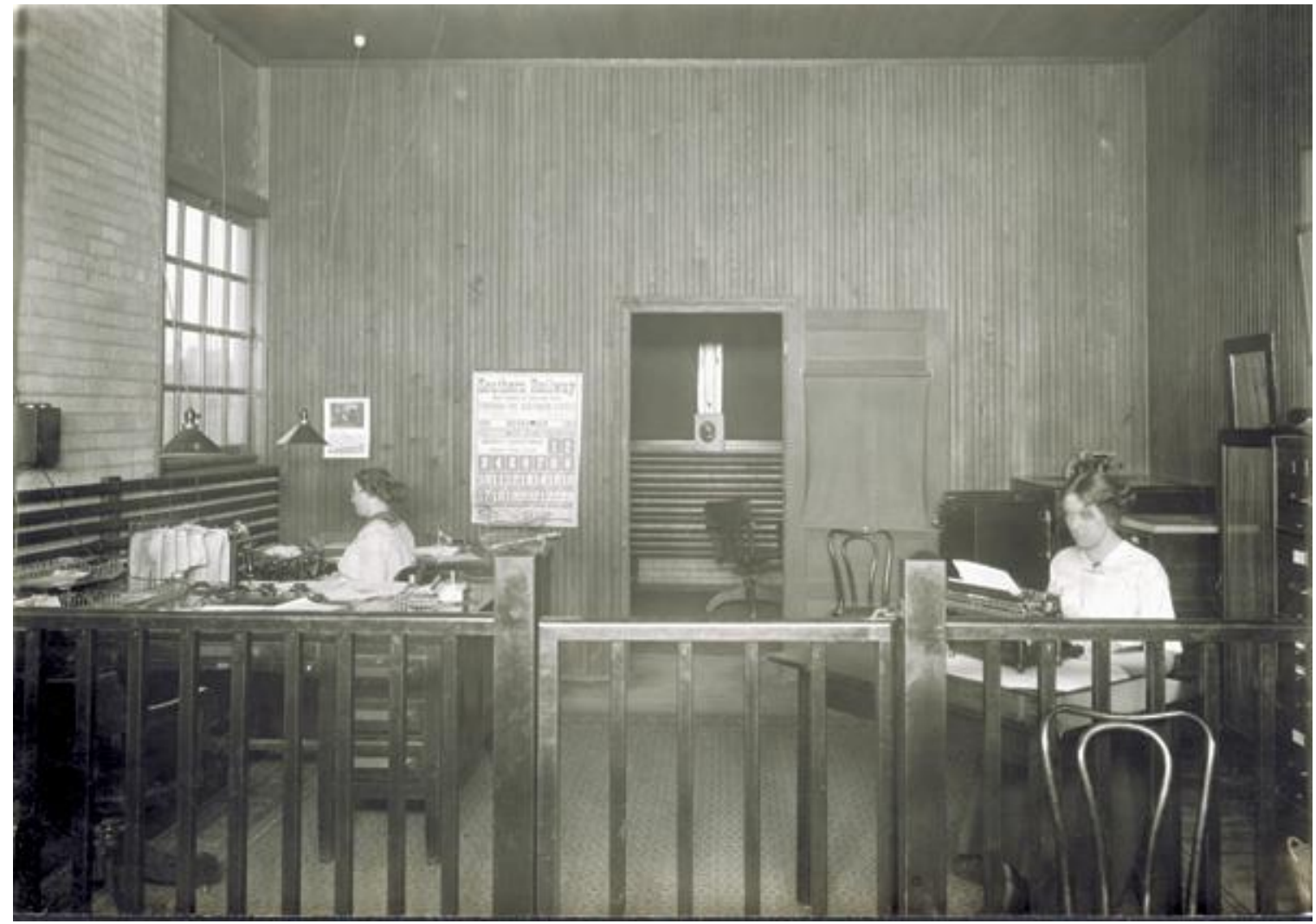

Figure 24. Front Office of Wright Company, 1911. http://corescholar.libraries.wright.edu/special_ms1_photographs/1445/

He explains that having the ability to zoom in on the calendar in the background enabled him to date other related materials to December 1911. He was also able to make probable guesses about the identities of the women in the photograph, with the help of supporting textual materials. 


\section{Historians use photographs to fill in under-represented histories.}

Another mode of historical evidence used by participants is filling in underrepresented histories or provide critical missing details. McCormack's research examines how dog sled technologies evolved in $19^{\text {th }}$ and $20^{\text {th }}$ centuries in the western Subarctic in Canada. She uses archival prints digitized by the Hudson Bay Company Archives (see Figure 25) as evidence about how dog sleds were constructed, a topic which she says is largely absent from written descriptions at the time period.

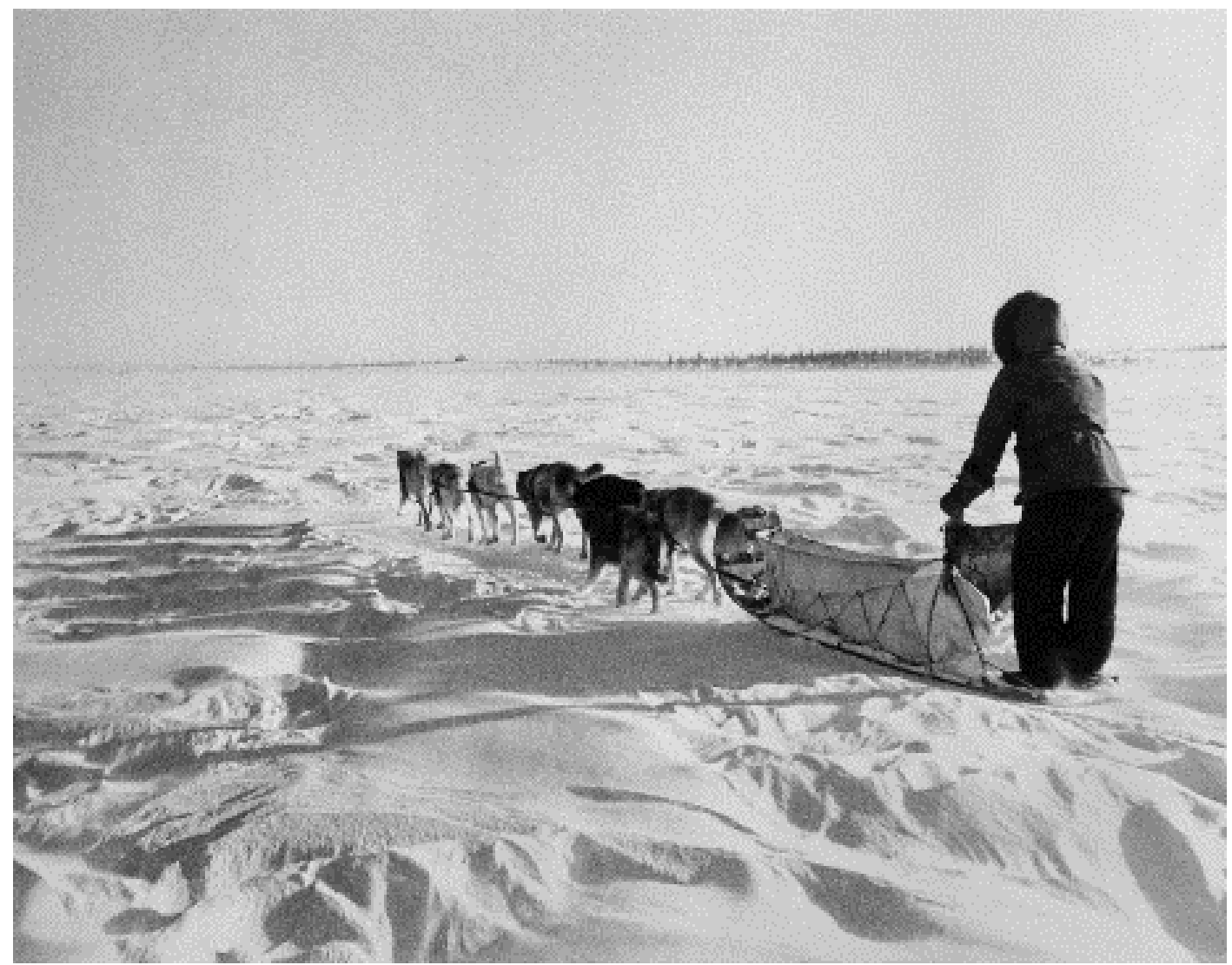

Figure 25. Dog Sled, Richard Harrington, 1947.

Courtesy of Hudson's Bay Company Archives, Provincial Archives of Manitoba, HBCA 1987/363-D-19A/10

She explains:

One of the things that happens with these sleds is that they start out as toboggans and over time they get wrappers and then eventually they get a backboard which is that part that goes on the back when you've seen pictures where people stand on the back driving dogs. I've been really interested in the evolution of that backboard and the archival picture I sent you is from the Hudson Bay Company and it shows you the dog sled being 
driven by, I think, five dogs and the backboard, so it was ...it's almost impossible to find these things described in the literature... people take this stuff for granted, that's where I find photographs particularly useful and then it also helps me think about timing.

Interestingly, discovering digitized archival photographs like these has led McCormack to conclude that popular $19^{\text {th }}$ century sketches and illustrations of dog sleds were mostly inaccurate.

Roach, a public historian working at the Dayton Aviation National Park, uses digitized photographs dating from 1948 in his work renovating and reconstructing Hawthorn Hill, the former home of the Wright Brothers (see Figure 26).

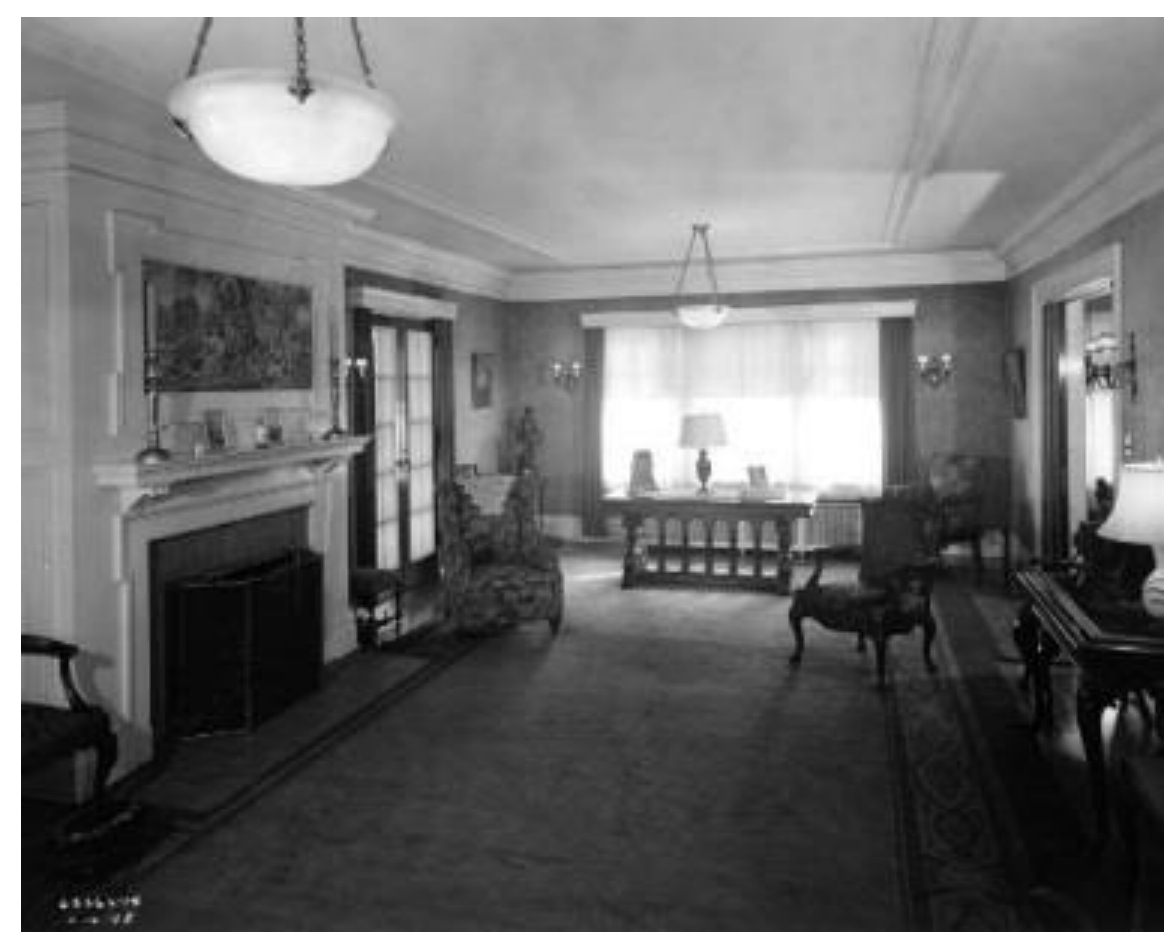

Figure 26. Hawthorn Hill Living Room, 1948.

https://www.nps.gov/parkhistory/online_books/daav/hawthorn_hill_hfr.pdf

Roach notes the importance and utility of digitized photographs, which offer "really our only source to understand, perhaps, how Orville lived in the space and to discuss his life in the house with visitors." His team used different interior photographs as primary sources to produce a 
historic furnishings report recommending how to model each room so that it resembled its 1948 appearance. ${ }^{372}$

As part of their visitor tours, Hawthorn Hill incorporates enlarged versions of historical photographs at various locations within the house. For example, an enlarged image of Orville Wright's chair (see Figure 27), which he modified following an airplane crash in 1908 to accommodate sciatica, is shown alongside the chair itself. Roach asserts that seeing in greater detail how Wright tinkered with his chair to make it more comfortable can help visitors "make a better intellectual connection" to the exhibit.

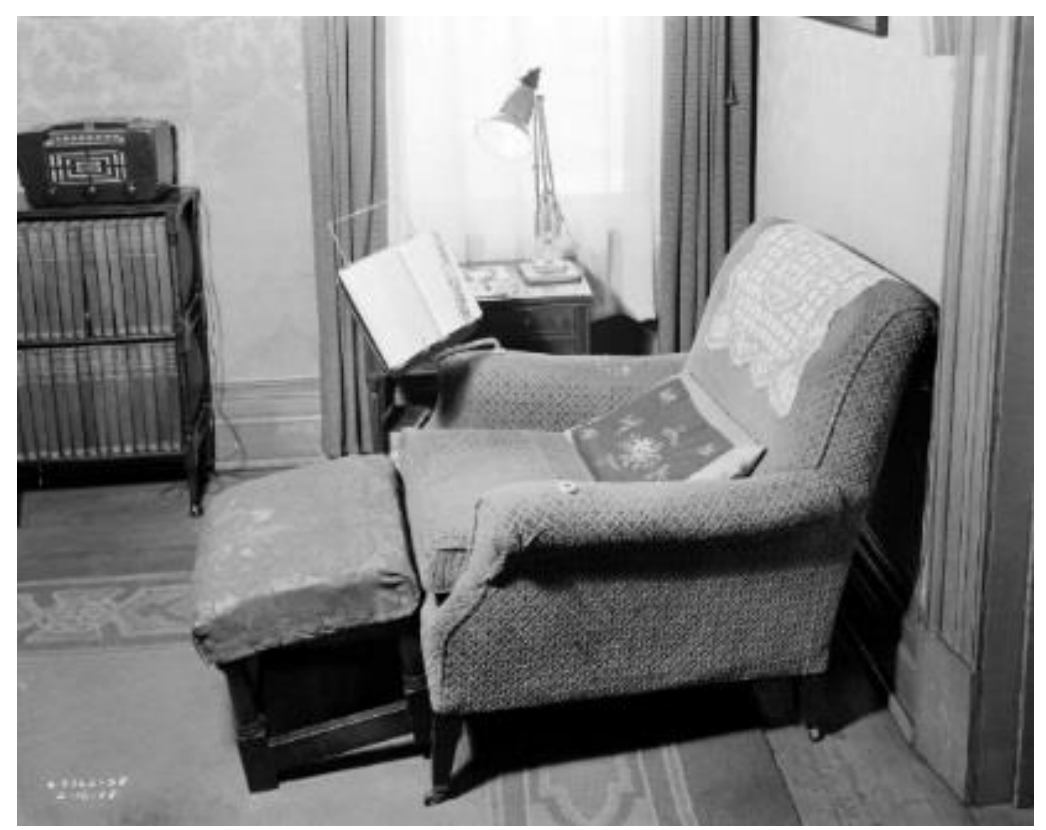

Figure 27. Orville's reading chair, February 16, 1948. NCR Archive at Dayton History

Roach was also able to use digitized photographs to fill in historical details for his book The Wright Brothers, The Wright Company: From Invention to Industry. He used photographic captions to help identify people working at the Wright Company. He explains:

The company's papers are scattershot really. You can't always depend on them to tell you what's going on or who's working for the company. There are no employee lists left,

\footnotetext{
372 Mary Grassick, Hawthorn Hill: A Furnishings History and Recommended Plan, Harpers Ferry, WV: Media Services, National Park Service (2007). https://www.nps.gov/parkhistory/online_books/daav/print_shop_hfr.pdf.
} 
in the papers. There are no pay registries or anything like that. So to the extent that I could identify the people it was from the photo captions.

In cases where historians are not able to locate exact matches for their topic of research, they may seek related images that are similar in location, time period and/or photographic content. Two of my participants used photographs from nearby geographical locations as historical documentation to investigate the evolution of a particular kind of technology. For example, Salo had difficulty locating certain relevant materials in his dissertation examining the evolution of ferry boats in the South Carolina low country. Since many ferries were constructed by individuals (what Salo terms "vernacular work"), he was unable to find architectural plans for how they were constructed. He knew certain types of ferries existed because of historical records but he was unable to find visual representations. In particular, he was searching for information about the rope-and-pull ferry (known as the rope ferry). He explains in his dissertation that "as with many topics related to ferries, one has to look at unconventional sources for information about the rope ferry..." ${ }^{373}$

Salo expanded his search to include depictions of ferries in nearby states, thinking that ferries from the same time period might share similar construction. He eventually found a photograph that depicts a rope bridge ferry boat on the Ocklawaha River in Florida (see Figure 28).

\footnotetext{
${ }^{373}$ Edward Salo, "Crossing the Rivers of the State: The Role of the Ferry in the Development of South Carolina, circa 1680-1920s," (PhD diss., Middle Tennessee State University, 2009): 149.
} 


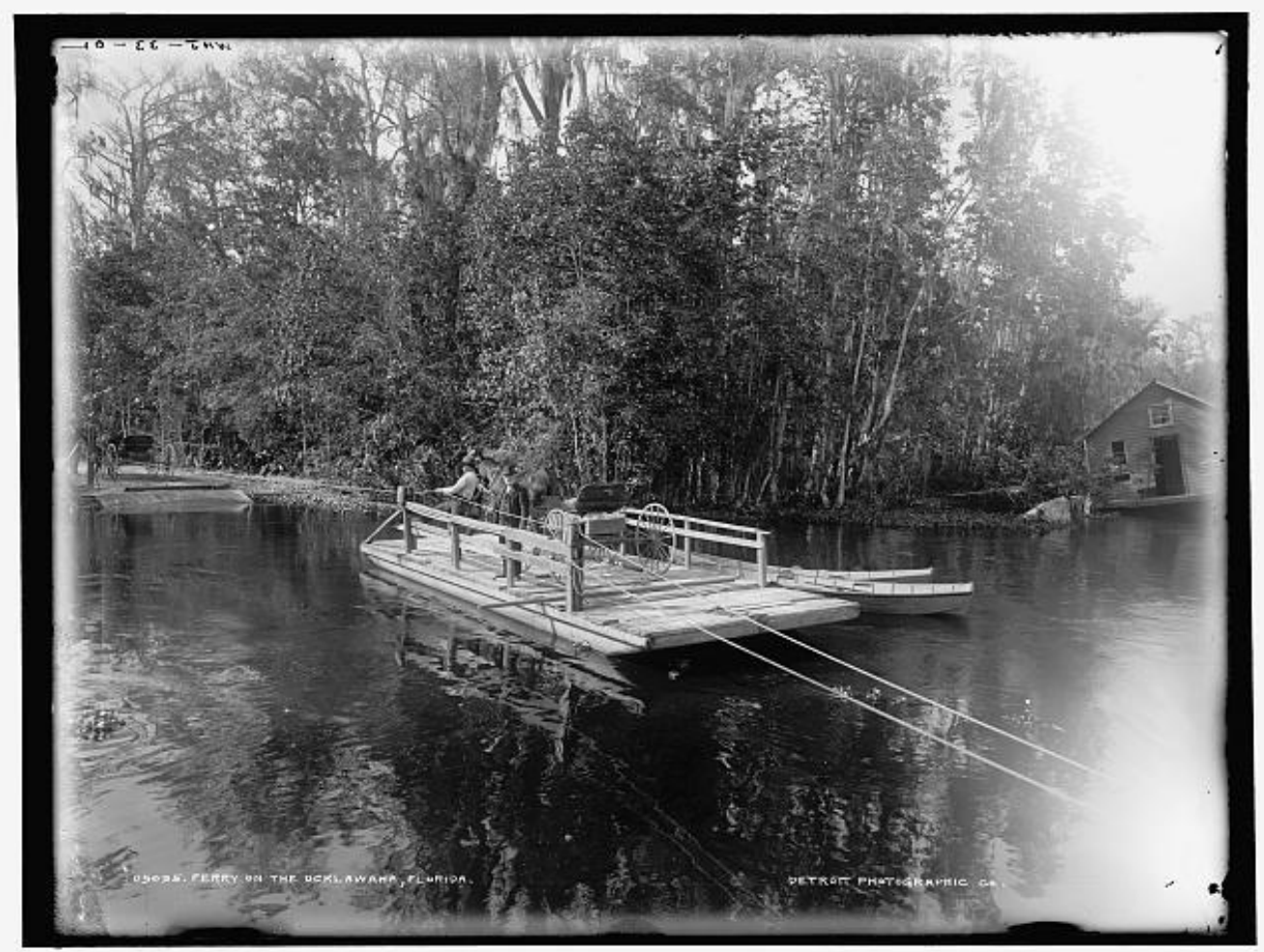

Figure 28. William Henry Jackson, Ferry on the Ocklawaha, 1902. https://www.loc.gov/item/det1994004870/PP/

He describes how he found the image using the online American Memory Collection from the Library of Congress:

Yeah I got to the point where I was just using the main photo collections, and I was just looking at ferry boats. I would go through South Carolina, North Carolina, Georgia, Florida, you know I was just trying to stay in that little region. And then when I was done with one state, I would go on to the next, you know? So yeah, it was completely a hunting and pecking kind of thing to find them.

Salo uses this image in his dissertation as a representation of what a rope bridge ferry operating in South Carolina from that time period might have looked like.

Historians also use digitized photographs for gathering information about the presence or absence of certain markers. As part of her research, for example, Clark wanted to find an approximate physical location of a Tennessee community called Catfish Bay. She only had one only historical reference to aid her, an 1873 history of Memphis which made mention that this particular community was located north of the Memphis and Charleston railroad depot. Once 
Clark found a particular photograph (see Figure 29), she was able to use it in conjunction with other visual sources to make the assertion that: (1) this depot existed and (2) that it was the depot mentioned in reference to Catfish Bay.

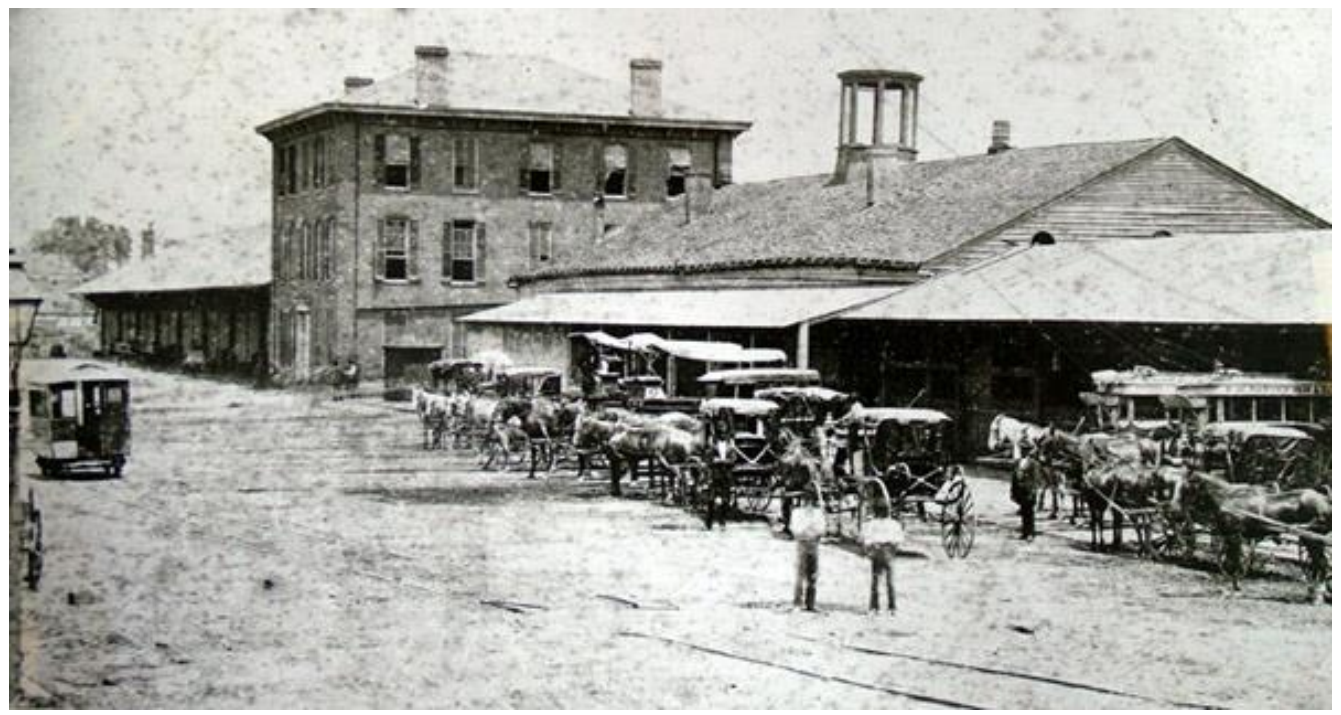

Figure 29. Memphis and Charleston Depot, ca.1880.

http://memphislibrary.contentdm.oclc.org/cdm/singleitem/collection/p13039coll5/id/907/rec/1

She explains her process below:

And I was looking for the location of Catfish Bay and it mentioned that it was near this particular depot. And so I had the map, and again it was really unclear how the depot was oriented on this map. So, what I was able to do then - once I found this image - was I was able to look at this in comparison to the map and see if I could find any landmarks. I didn't necessarily find any landmarks, but I feel like I'm getting closer to the location. And I may never actually find the exact location, but at least I have more clarity that there really was a depot. Evidence that there's a depot.

Clark also uses Figure 30 to make assertions about the time period. For example, she points out that the absence of cotton in the image is quite unusual. This absence, combined with the image's emphasis on passenger carriages, suggests to Clark the possibility that this was a promotional photograph used to signify a "transition to the railroad as a transporter of people instead of goods." 


\subsection{A Conceptual Framework for Studying Historians' Image Use}

A central challenge in designing and conducting this research study was the absence of conceptual models or frameworks to address my overall research questions. In particular, I was not able to find specific methdologies for eliciting descriptions from individuals as they interpretted and evaluated visual materials in digital environments. Photo-elicitation interviewing techniques proved useful as stimulus for generating descriptions of experiences, but significant aspects of the interaction were likely lost in translation. Additionally, the historiographical literature provided few examples regarding how historians interpret, evaluate, and decide to use (analog or digital) photographs as forms of evidence.

Toward that end, this study introduces an exploratory, conceptual framework that can be used for studying historians' scholarly image use as a multi-faceted occurrence (see Figure 31). The framework focuses attention on the six analytical themes that take place at two different junctures in historians' experiences: potential image use and evidential image use.

Distinguishing between these two spheres proved to be a helpful strategy for addressing the complexities of information use in digital research environments, which are continuously mediated by a confluence of techno-social and spatiotemporal factors. Rather than focusing on image use as the sole outcome, this framework permits exploration of activities, practices, and processes that happen as historians decide to use (or not to use) materials further. It presents a conceptual approach for attending to interactions in digital research environments, enabling a holistic understanding of criteria that historians employ around their image selection and use. 


\section{A CONCEPTUAL FRAMEWORK FOR STUDYING HISTORIANS' IMAGE USE}

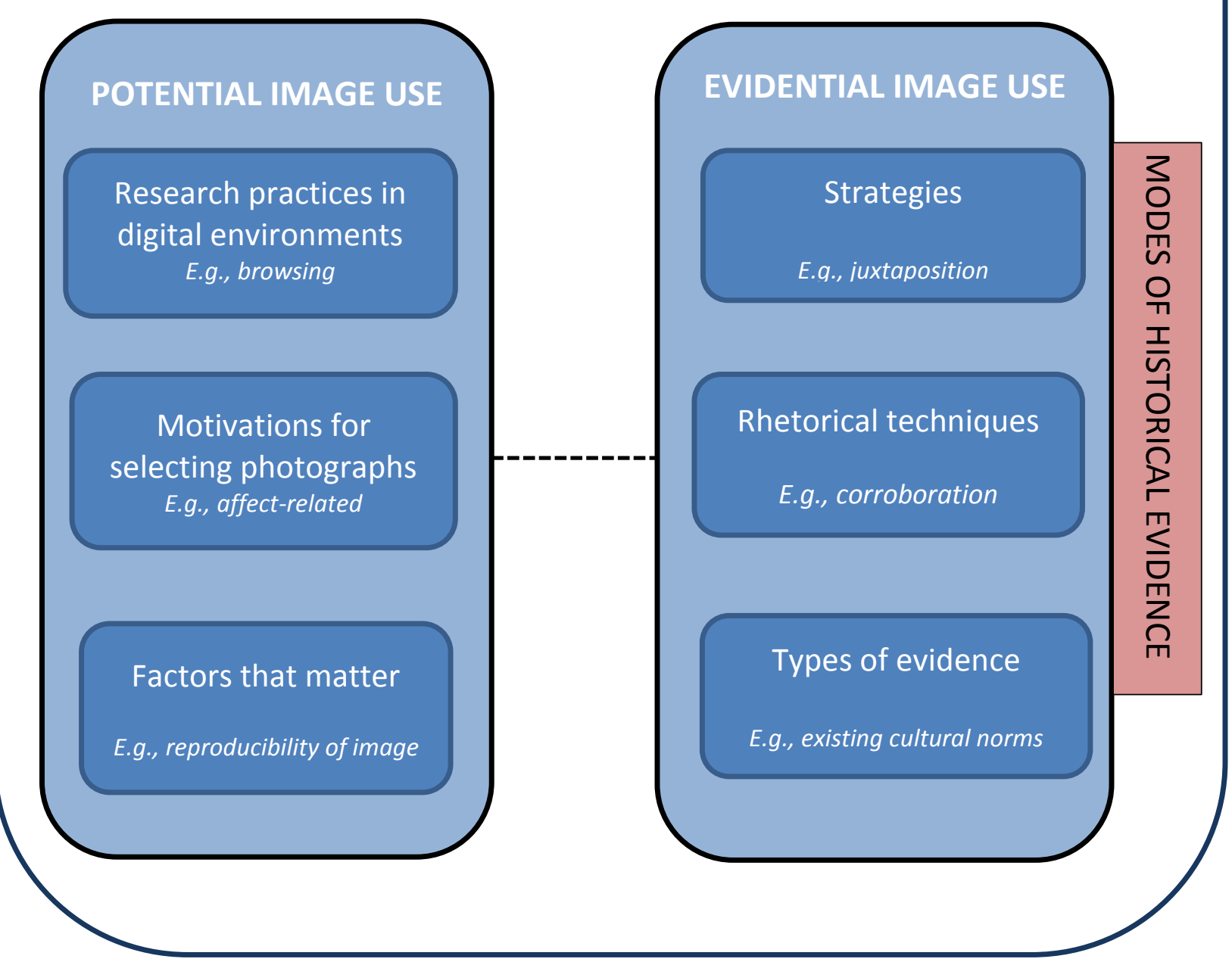

Figure 30. A conceptual framework for studying historians' image use 


\section{CHAPTER 5: CONCLUSION}

This dissertation examines historians' experiences using digitized photographs as evidence, to reveal the factors that matter to them in their decision to use (or not to use) materials. Using thematic analysis, I was able to surface descriptive themes that characterized historians' experiences in digital research environments. Since the focus of my study was about historians' use of digitized photographs, my findings are tailored toward interactions in digital environments.

In this chapter, I situate my findings within the existing literature and discuss implications and future work. First, I discuss the significance of this study in relation to the extant literature, comparing and contrasting my findings within a discussion of how they contribute to the literature. Next, I describe some of the limitations of this study and suggest future empirical endeavors. Finally, I discuss practical implications for archival professionals seeking to provide access to digitized photographs.

\subsection{Significance of the Study}

In this dissertation, I examine self-identified historians' experiences to reveal the factors and qualities that matter to them in their interactions in digital environments as they decide to use (or not use) digitized photographs. This study contributes to the extant literature in three significant ways.

First, it addresses absences in both the historiographical and archival literature regarding how and why historians use digitized materials as evidence in their scholarship. Few empirical studies have explored the historical process of constructing evidence from primary source 
materials. The findings in this research helped to identify the practices and processes underlying the construction and use of photographs as forms of evidence. My respondents employed strategies and rhetorical techniques alongside their own interpretation of digitized photographs. The case vignettes presented in section 4.4.2 illustrate a broadening of Wineburg's notion of historical thinking ${ }^{374}$, showing how my participants' own interpretive ideologies influence how they construct historical arguments. My findings also suggest that there are contextual, ecological, and social factors related to their interactions that influence their potential use of materials. In studying the overall experiences of my participants in digital environments, I was able to assess the significance of those interactions.

Second, this research presents exemplary models for two sets of disciplinary scholars. The vignettes provide descriptive summaries that may be useful for historians interested in analyzing and using photographs as visual evidence. At the same time, information professionals can also use the case vignettes as the basis for empirical studies aiming to understand potential and evidential use of archival materials as forms of evidence.

This research also contributes to the larger literature on information use, particularly related to visual materials. It highlights the benefits of considering potential use as a separate stage of research in which potential interactions in digital environments influence selection activities. It also introduces the notion of "perceived usefulness" that can be used as a construct for granular perspectives on sociotechnical constraints and affordances characterizing scholarly interactions. Lastly, it explores how and why historians use digitized photographs in constructing modes of historical evidence.

\footnotetext{
${ }^{374}$ See: Samuel Wineburg, "Historical Problem-Solving: A Study of the Cognitive Processes Used in the Evaluation of Documentary Evidence" (PhD diss., Stanford University, 1990); Samuel Wineburg, "Historical Problem Solving: A Study of the Cognitive Processes Used in the Evaluation of Documentary and Pictorial Evidence." Journal of Educational Psychology 83, no. 1 (1991): 77.
} 


\subsection{Limitations of the Study}

There are numerous limitations in this study. One limitation concerns the relatively small sample size $(\mathrm{n}=15)$. There are no strict guidelines regarding sample size in qualitative case study research; instead, the emphasis is on the information-richness of cases. Toward that end, I used purposeful sampling to target information-rich cases and observed the principles of data saturation in my data collection. Patton points out that in case study research, "in-depth information from a small number of people can be very valuable, especially if the cases are information-rich." ${ }^{375}$

Another limitation in this research concerns the interdisciplinary nature of my sample population, making it impossible to generalize my findings. Despite using the umbrella term of "historian," the majority of my study participants are not currently teaching in academic history departments (see Table 2). It was necessary to expand my sample population for a number of reasons. Initially, it was difficult to locate individual historians working in academic history departments that were using digitized photographs to make historical arguments. Through informal conversions conducted in the early stages of my research, I learned that institutions were not yet formally tracking scholarly use of their digital collections which prevented identification of potential recruits. ${ }^{376}$ My particular focus on studying scholars using photographs from these collections (as opposed to all visual materials) proved to be an additional challenge.

\footnotetext{
${ }^{375}$ Patton, 184.

${ }^{376}$ Throughout 2010-2014, I had conversations about current practices measuring digital collection use with the following individuals: Laura Clark Brown from the Southern Historical Collection at the University of North Carolina Chapel Hill; J. Stephen Catlett from the Textiles Teachers \& Troops LSTA Project at the University of North Carolina Greensboro; Stephen Fletcher from the North Carolina Collection Photographic Archives at the University of North Carolina Chapel Hill; Barbara Natanson from the Print and Photographs Division at the Library of Congress; and Ashley Reed from the William Blake Archive at the University of North Carolina Chapel Hill.
} 
In the pilot study I conducted in 2011, my sample population consisted of academic historians working in the United States. ${ }^{377}$ The goal of that research was to explore historians' current research practices in the midst of technological changes. My findings prompted new questions about historical practices in digital environments. In particular, I concluded that future research should explore the "factors and qualities that influence historians' interactions with primary source materials." ${ }^{378}$

This dissertation research aimed to build on these findings by exploring historians' interactions with, and use of, one group of primary source materials - digitized archival photographs. Since I was most interested in how and why historians use digitized images as forms of evidence, expanding my sample population to encompass self-identified historians doing historical work allowed me to gather important aspects of those interactions while providing me with an adequate number of participants for case study research. Arguably, the rise of interdisciplinary fields such as the digital humanities suggests that maintaining disciplinary boundaries in today's academic climate may be less relevant than before. Indeed, I found great value in addressing the broad practices of individuals doing historical work with photographs, recognizing that my sample had unique qualifications and training. In fact, though most of my participants were not currently teaching in academic history departments, eight had received their doctorate from "traditional" history departments.

Another limitation concerns the overall lack of generalizability in case study research. Accordingly, my findings in this study cannot be treated as a representative sample from a population. For example, it would be inappropriate to infer that my findings describe how and

\footnotetext{
377 Alexandra Chassanoff, "Historians and the Use of Primary Source Materials in the Digital Age," The American Archivist 76, no.2 (2013):458-480.

${ }^{378}$ Ibid, 472.
} 
why all historians use digitized photographs as evidence. As such, the conceptual framework presented in 4.5 is best interpreted as a set of descriptive and analytical themes that can be used as a baseline for exploring historians' image use. According to Bryman, "case studies should be evaluated in terms of the adequacy of the theoretical inferences that can be generated. The aim is not to infer findings from a sample to a population, but to engender patterns and linkages of theoretical importance." ${ }^{\prime 379}$ As a precaution, Yin recommends returning to a study's main theoretical propositions to generalize findings. ${ }^{380}$ In this research, two main theoretical propositions guided my investigation.

1) Information is a phenomenon that emerges in specific contexts.

2) Information use is always situated within contextual, ecological, and social practices. As such, we cannot understand the "how" of information use without attending to the practices which constitute the experience.

Both of these propositions guided my analysis and interpretation of themes within and across cases.

A third limitation relates to my data collection procedures. The goal of my research was to explore interactions underlying historians' experiences using digitized photographs. However, since my sample population consisted of historians who had already used photographs as historical evidence, their experiences were necessarily relayed retrospectively during photoelicitation interviewing. Thus, their original interaction is not represented in this study. I attempted to address this limitation by asking participants, whenever possible, to pull up the original website during the photo-elicitation portion of the interview.

A final limitation in this study concerns implicit researcher bias in translating the specific examples of historians' use into different modes of historical evidence. I do not have training in

\footnotetext{
${ }^{379}$ Alan Bryman, Research Methods and Organization Studies, London: Unwin Hyman (1989): 173.

${ }^{380}$ Yin, Case Study Research, 10.
} 
historiography or visual studies; I established and distinguished different modes based on discursive analysis and inductive reasoning. As noted earlier, the interdisciplinary nature of my sample means I cannot generalize these modes more broadly to historical practices.

\subsection{Implications and Future Work}

The circulation and use of digitized primary source materials has grown significantly in the $21^{\text {st }}$ century. Archival professionals must engage with these changes in digital scholarship at multiple levels, from acknowledging new scenarios for access to thinking about creative methods for encapsulating the provenance of images. This dissertation research addresses a noted gap in the literature, exploring how one particular group of scholars - self-identified historians using digitized archival photographs - interpret and use materials as evidence in their scholarly activities.

How can archival information management systems best accommodate historians' evolving information needs amidst such changes? A particular challenge lies with the difficulty in describing visual materials for further use; photographs can have many interpretations. Such range in interpretive possibilities has important ramifications for archival description and access. Shatford-Layne neatly summarizes the challenge: "If images may be defined as referents for the sense of the words used to describe them, it seems clear that we cannot expect images to translate neatly and simply into words." What is to be done?

The results of this research suggest that distinct material practices remain critical to historians' image selection and use. For example, despite potentially finding an image via Google Image Search, my respondents stress the importance of downloading photographs from websites affiliated with archival institutions, to help ensure trustworthiness of materials. Within their environmental interactions, functional aspects such as their ability to download images at different resolutions also play a significant role in determining further use. Respondents indicate 
that they want as much original descriptive information (for example, the handwritten captions on the back of photographs) to be made accessible as possible. They also suggest that providing additional description about the digitization environment, including the documentation of archival decision-making related to the overall collection, would be beneficial.

At the same time, archives should also present flexible and fluid options for access and use of archival photographs. An explicit goal for design should be the development of archival environments that embrace and support fluid interactions. In particular, performative approaches to interface design, such as those suggested by Johanna Drucker, can orient archives towards constructing access systems that more closely represent "an event-space of interpretive activity."381 Rather than designing for specific access points or improving information retrieval strategies, archival professionals should consider image use in digital environments as a multifaceted, multi-layered experience in which meaning is constructed (or not) through a series of complex mediations.

Indeed, recasting information use as information experiences can shed light on what makes interactions meaningful for scholars in digital environments, in ways that traditional measurements of information use fall short. Indeed, the fact that my respondents are regularly using digital sources is not (yet) widely reflected in either the historiographical or LIS literature. Numerous explanations for this discrepancy point to the need for developing better methods for capturing use. For example, recent LIS empirical research on historians' information use focuses on journals and does not include monographs. ${ }^{382}$ Citation analysis, a frequently used measurement source, may

\footnotetext{
381 Drucker, "Performative Materiality."

382 In analyzing trends in historians' image use, Harris and Hepburn examined articles from prominent historical journals to track use. They found no discernible growth or decrease in image use. However, historians' primary research output remains the production of monographs. See: Harris and Hepburn, "Trends in Image Use."
} 
not provide a representative understanding about historians' use of digital materials; some historians may consult materials to structure their argument, but leave them out of publication due to copyright or quality concerns. Moreover, citation practices for digitized materials in archives are still evolving. There are no standardized recommendations for citing digitized items across citation styles, making citation analysis difficult. The Web of Science, a frequently used tool in citation analysis, does not allow citation searches by material type. Therefore, the number of times a digitized photograph has been cited in research materials is not easily obtained without implementing additional scripting. Further exploration and understanding of reproduction issues, a critical part of the use equation in digital environments, is still needed.

Historians continue to employ non-linear, non-sequential practices when conducting research in digital environments. However, in contrast to previous work in LIS studying historians' practices, this research identified two distinct spheres, potential image use and evidential image use, that can be used to characterize how and why historians decide to use (or not use) images. Isolating these activities, processes, and practices into separate spheres allowed me to explore dimensions that matter in their experiences.

In general, historians perceive the digitization of materials as beneficial. The more information that a repository provides online, the better. Kate Sampsell-Willmann says: "You know, having everything, having all the captions and having everything means you don't actually have to visit the repository. We can do it from a distance." However, one concern shared by respondents is the impact of encountering digitized photographs apart from their originating archival collections. 
Indeed, this research found a potential shift in where historians are locating photographs for use in their scholarly activities. Only one participant mentioned finding aids at all, while eight participants used Google image search. The emergence of different kinds of digital collections (amateur/hobbyist online collections and personal digital archives amassed by researchers) suggests that the institutional archive may no longer be the starting point for image search. While trust in source material remains a central concern for historians, whether and how they evaluate photographs located exclusively through Google image searches has not yet been explored. Standardizing practices around the embedding of metadata into digitized photographs as well as tracking use throughout the lifecycle of digital images remain two crucial areas of future research.

Although the study sample was not designed to be representative of all historians, the findings in this study do suggest that historians may be more comfortable using photographs as primary sources than previously anticipated. A significant number (thirteen) use digitized photographs as the primary source of evidence in crafting their argument- in contrast to many claims made in the literature. Several participants in this study mention using photographs as their initial starting point, building and constructing evidence. For example, Gabriel describes her philosophical approach in using images for teaching:

So I, as a historian, use the image to tie into the broader methodological agenda of what I'm trying to teach. So the image is deeply integrated into the rest of the conversation. So the image is not supplementary evidence, it's integral to what the main purpose of the lesson is.

In constructing different modes of historical evidence, historians draw on their own perspectives and ideologies. One interesting point that became clear to me is that, in their process of interpreting and using photographs as evidence, historians presume a perspectival orientation that enables them to effectively construct their argument. For example, a historian 
might use a photograph as representational of 1960s' cultural norms. In constructing their mode of evidence, this historian might claim that the original photographer intentionally captured cultural norms, or they might claim that the photograph itself is capable of representing cultural norms. In both instances, a certain perspective orients the historian in how they approach image use. Nearly all of the historians in this study used photographs to construct arguments that serviced a cast of spectators (e.g., photographic subjects, the public perceiving the photograph at the time it was taken), each of whom were involved in either the production and/or consumption of the photograph.

While the advent of digitization has introduced new possibilities, the extent to which specific scholarly practices have fundamentally changed has been underexplored. Future research seeking to understand how, and whether, these differences have influenced interpretative practices would prove useful. For example, one study could compare and contrast how historians perform essential practices like source criticism in print and digital environments.

The participants in my research suggest that historians and archivists should work together to better anticipate information needs of image users. Krase explains:

So, what tends to happen in our academic world is that people stay amongst themselves, right? So it would make sense for people who are going to be doing, or already doing these sorts of things [cataloging and organizing visual materials], to be going to the American Historical Association meetings, go to the American Studies Association, this one and that one, etc. And to at least peruse the journals, etc., to see how the images are being used. Because if they get a sense of how they're being used, they might have a better sense of how they can be organized for searches.

Sampsell-Willman concurs, albeit with the goal of enriching the process of photographic analysis through "a concerted effort among historians and art historians to research, catalogue, analyze, and explain the relevant intellectual biographical details that will allow a deeper understanding 
of the individual photographer to emerge. ${ }^{383}$

It seems that historians and archivists would benefit intellectually from working together closely, to share information with each other about work practices. Participants in my research seemed interested in learning more about the appraisal and processing decisions archivists make. Learning more about how historians evaluate visual sources could certainly benefit archival practitioners (and was the overarching goal of this study). A recently developed methodological approach is digital historiography, which Joshua Sternfeld defines as the "interdisciplinary study of the interaction of digital technology with historical practice." 384 For Sternfeld, the convergence of traditional historiographic principles with archival theory provides a useful, common methodological foundation for archivists and historians working with digital archival materials, or what he terms "digital historical representations." Digital historiography asks practitioners from both fields to consider what mediating factors help transform digital historical representations into sources of historical evidence.

My findings also suggest that image use can be motivated by affective factors such as empathy. Though emotion is a relatively recent research area of interest in LIS, exploring links between affect and information behavior in digital environments is an exciting area for future work. For example, research studies exploring the differences in affective responses to digital and print photographs would prove particularly interesting in further developing understandings of interpretation and meaning-making in digital environments.

Making meaning from archival photographs is a complex process, regardless of their digital or analog status. Writing in 1988, Margolis asserts, "In fact, the meaning of a particular

\footnotetext{
${ }^{383}$ Kate Sampsell-Whillmann, Lewis Hine as a Social Critic, 9.

${ }^{384}$ Joshua Sternfeld, “Archival Theory and Digital Historiography: Selection, Search, and Metadata as Archival Processes for Assessing Historical Contexutalization," The American Archivist 74 (2011): 544.
} 
photograph typically is generated as much by the context in which it is found - site, collection, date, photographer, caption - as by the image itself." ${ }^{385}$ Margolis highlights the importance of studying spaces of interaction as critical places where meaning-making happens. In particular, conceptualizing the performative space of information experience(s) provides a useful framing device for interrogating meaningful interactions in digital environments.

In this research study, mediating factors from different social, contextual, and ecological domains impact how and why historians use digitized archival photographs as evidence. Influential factors range from how scholars first notice and appraise materials down to the kinds of obstacles that prevent them from using materials further. The value in studying information experiences with digital materials is that it can reveal at the moment of interaction how information is constituted by the interaction and performed rather than focusing on defining what information is. The latter is well-trodden territory, but orienting toward the former is critical for developing new understandings of, and literacies for, how we interpret and use information in hybrid research environments.

\footnotetext{
${ }^{385}$ Margolis, Radical History Review, 36.
} 


\section{APPENDIX A: INTERVIEW RECRUITMENT POST}

Date

Dear Colleagues,

Are you a (self-identified) historian? Have you used digitized versions of archival photographs in your teaching activities, publications, presentations, or related research pursuits? If so, please consider participating in an interview session as part of my doctoral research.

The interview should take approximately one hour and can be conducted in person, over the telephone, or using Skype. Your responses to these questions will be kept confidential. There is no compensation for participating in this study; however, I am confident that your participation will contribute significantly to this emerging area of research.

If you are willing to participate, please send an email to: achass@email.unc.edu to confirm your interest. I am happy to answer any questions for you as well.

Again, thank you so much for considering participating in this research.

Sincerely,

Alexandra Chassanoff

Please note: This study has been approved by the University Institutional Review Board (\#150566). 


\section{APPENDIX B: INTERVIEW RECRUITMENT EMAIL}

Date

Dear

I am a doctoral student conducting my dissertation research on how historians are using digitized photographs as forms of evidence. I am writing to ask you to participate in an interview session with me to discuss how and why you have used images in your research.

Few empirical studies have explored historians' use of visual materials as forms of evidence. As a historian who has used images in your scholarship, you are in an ideal position to provide valuable first-hand perspectives on your experiences.

The interview should take approximately one hour and can be conducted in person, over the telephone, or using Skype. Your responses to these questions will be kept confidential. There is no compensation for participating in this study; however, I am confident that your participation will contribute significantly to this emerging area of research.

If you are interested and able to participate, please email me confirmation of your interest no later than ___ I I am happy to answer any questions for you as well.

Again, thank you very much for considering participating in this research.

Sincerely,

Alexandra Chassanoff

Please note: This study has been approved by the University Institutional Review Board (\#150566). 


\section{APPENDIX C: FACT SHEET}

University of North Carolina-Chapel Hill

Consent to Participate in a Research Study

Adult Participants

Social Behavior Form

IRB Study \# 15-0566

Title of Study: Historians and the Use of Digitized Photographs as Evidence

Principal Investigator: Alexandra Chassanoff, Doctoral Student

UNC-Chapel Hill Department: Information and Library Science

UNC-Chapel Hill Phone Number: (919) 962-1345

Faculty Advisor: Cal Lee

Faculty Email Address: callee@ilis.unc.edu

Study Contact Telephone Number: xxxxxxxxxxx

Study Contact Email: xxxxxxxxxxxxx

\section{What are some general things you should know about research studies?}

You are being asked to take part in a research study. To join the study is voluntary. You may refuse to join, or you may withdraw your consent to be in the study, for any reason, without penalty. Research studies are designed to obtain new knowledge. This new information may help people in the future. You may not receive any direct benefit from being in the research study. There also may be risks to being in research studies.

Details about this study are discussed below. It is important that you understand this information so that you can make an informed choice about being in this research study. Please keep a copy of this information sheet. You should ask the researchers named above, any questions you have about this study at any time.

\section{What is the purpose of this study?}

This research study intends to explore the information practices of historians using digitized photographs in their research endeavors. In particular, I am interested in understanding how historians interpret, evaluate, and use digitized photographs as forms of visual evidence.

\section{Are there any reasons you should be in this study?}

You should participate in this study if you are a self-identified historian who has used digitized archival photographs in teaching activities, publications, presentations, or related research pursuits.

\section{How many people will take part in this study?}

If you decide to be in this study, you will be one of approximately 15-20 people in this research study.

\section{How long will your part in this study last?}

You will be asked to participate in one interview session, lasting approximately 1-1.5 hours. At the close of the interview, you will be asked if you are willing to participate in a short follow-up 
interview to further explore or clarify themes and issues raised in the original interview or from supplementary documents.

\section{What will happen if you take part in the study?}

At the beginning of each interview session, I will introduce myself, the purpose of the study, and obtain verbal consent. You will be asked to answer demographic information and a short questionnaire about your image use practices (see Questionnaire). In the second part of the interview session, I will present you with two digitized photographs that you selected for use in this interview. I will then ask you a series of questions relating to how you interpret, evaluate, and have used each photograph in scholarly activities (see PEI protocol). You do not have to answer any questions that you do not wish to answer, for any reason. With your permission, these interviews will be recorded for transcription. The recordings will be deleted after the transcriptions are created.

\section{What are the possible benefits from being in this study?}

Research is designed to benefit society by gaining new knowledge. Your participation is important to help us understand historians' experiences with digitized photographs as forms of evidence. While you may not benefit personally from being in this research study, you will be contributing to empirical research that seeks to better understand evolving information practices so that scholarly infrastructure can better support changing needs and habits.

\section{What are the possible risks or discomforts involved from being in this study?}

There are no risks anticipated should you participate in this study.

\section{How will your privacy be protected?}

Your name or other identifying information will be used in the presentation of this research to others and disclosed in the material that is published.

\section{Will you receive anything for being in this study?}

There is no financial benefit for your participation. The cost to participate is your time, and for that we are very appreciative.

\section{Will it cost you anything to be in this study?}

There are no costs for being in the study.

\section{What if you have questions about this study?}

You have the right to ask, and have answered, any questions you may have about this research. If you have questions, or concerns, you should contact me at (617) 501-3669 or by email at achass@email.unc.edu. You may also contact my advisor, Dr. Cal Lee at (919) 962-7024 or by email at callee@ils.unc.edu

\section{What if you have questions about your rights as a research participant?}

All research on human volunteers is reviewed by a committee that works to protect your rights and welfare. If you have questions or concerns about your rights as a research subject, or if you would like to obtain information or offer input, you may contact the Institutional Review Board at 919-966-3113 or by email to IRB_subjects@unc.edu. 


\section{APPENDIX D: VERBAL CONSENT SCRIPT}

The purpose of this research is to explore the experiences of historians using digitized photographs as forms of evidence in scholarship. Approximately 15-20 self-identified historians will be enrolled in this study. You will be asked to participate in an interview which should take between 60 and 90 minutes. Supplementary artifacts (course syllabus, conference presentations) will also be collected. At the close of the interview, you will be asked if you are willing to participate in a short follow-up interview to further explore or clarify themes and issues raised in our original interview or from supplementary documents.

There are no risks anticipated should you participate in this study. However, your name and other identifying information will be used in the presentation of this research to others and disclosed in the material that is published.

This study will be recorded for the purposes of transcription. This recording will be destroyed as soon as the transcription of this interview takes place. Do you consent to being recorded during this conversation? (If the person says yes, proceed. If they say no, ask: "Do you consent to notetaking during the interview?" If they say yes, proceed. If they say no, thank them for their time and explain that they are not eligible.) 


\section{APPENDIX E: SEMI-STRUCTURED INTERVIEW PROTOCOL}

\section{A. Demographic Information}

1. What is your current title?

2. Gender:

Male

Female

3. Age:

$$
\text { I prefer not to answer }
$$

\begin{tabular}{l}
$25-35$ \\
$36-45$ \\
$46-55$ \\
$56-65$ \\
\hline \\
Over 65 \\
I prefer not to answer
\end{tabular}

4. Number of years teaching history at a college or university:

5. Number of years teaching history at your current institution:

6. Primary courses you teach:

7. Primary area(s) of research:

\section{B. Image Use Practices}

Think about the research activities where you used digitized photographs.

Please describe the following:

8. Topic of research:

9. Chronological period (e.g., 1950-1965):

10. Year you started this research:

Year you ended this research or ongoing:

11. Main archives, special collections and repositories used in this research:

12. Can you describe what techniques you used when you were searching for [Image $\mathrm{x}]$ ?

a. [probe] used google image search; used digital library collections, etc.

i. [probe] what specific collections did you use?

ii. [probe] did you have specific image in mind? What point in the process?

13. [probe: read back list mentioned] What did you consider to be the most useful technique when you were searching for an image? 
14. How were you planning on using the images? For teaching? Presentation at a conference? In your research?

15. What are the factors that are most important to when you are deciding whether to use a digital image or not?

a. [probe] e.g., Trustworthiness of image repository; ability to access collection

i. [probe] How do you ensure that [factor $x]$ ?

ii. [probe] What other factors matter to you?

16. Are there particular types of images that resonate with you as forms of historical evidence?

a. [probe with examples]

17. What does "historical evidence" mean to you? 


\section{APPENDIX F: PHOTO-ELICITATION INTERVIEW PROTOCOL}

SCENARIO 1: Photograph selected by participant for use in teaching

Interviewer: You were asked to pre-select a digitized photograph you have used in teaching

1. Can you describe the techniques you used in your search for materials?

a. [probe] used google image search; used digital library collections, etc.

i. [probe] What specific collections did you use?

ii. [probe] Did you have specific images in mind?

2. [Read back list mentioned] Out of that list, what do you consider to be the most useful technique in your search? Why?

3. How did you find this image?

a. [probe] Did you look at other related materials in the collection?

i. [probe] Do you remember how you established any important details about this photograph?

4. Tell me more about how you would interpret this image.

a. [probe] What can you tell me about the context?

b. [probe[ What do you know (if anything) about the photographer?

c. [probe] Have you seen this image used previously as historical evidence?

d. [probe] Are there particular details related to the arrangement or composition of this photograph?

e. [probe] What is being represented? What is being excluded?

5. Why did you decide to use this image in your teaching?

6. What do you see as the most important factors in the presentation of this photograph?

a. [probe]: descriptive info - which are most useful?

b. [probe]: provenance and context?

c. [probe]: ability to zoom? quality?

7. Do you trust that this photograph is an accurate historical representation? Why or why not?

8. Who do you think was the original audience(s) for this image? 
SCENARIO 2: Photograph selected by participant for use in research

Interviewer: You were asked to pre-select a digitized photograph that you have used in research.

9. How did you find this image?

a. [probe] Did you begin your search for this particular image or more broad idea?

10. Please describe how you used this image in your scholarship.

a. [probe] publication or presentation? Illustration, primary source of evidence, etc? i. [probe] illustration, primary source of evidence, etc.

11. Tell me more about how you would interpret this image.

a. [probe] What can you tell me about the context?

b. [probe[ What do you know (if anything) about the photographer?

c. [probe] Have you seen this image used previously as historical evidence?

d. [probe] Particular details about arrangement in photograph - significance of relationships

e. [probe] Who do you think was the intended audience for this photograph?

f. [probe] What is being represented? What is being excluded?

12. Why did you decide to use this image in your scholarship?

a. [probe] Are there specific factors that might prevent you from using this image as a form of evidence?

i. [probe] trustworthiness of image repository, ability to access collection

13. What do you see as the most important factors in the presentation of this photograph?

a. [probe]: descriptive info - which are most useful?

b. [probe]: provenance and context?

c. [probe]: ability to zoom? quality?

14. Do you trust that this photograph is an accurate historical representation? Why or why not?

15. What does "historical evidence" mean to you? 


\section{BIBLIOGRAPHY}

Akker, Chiel van den, Susan Legene, Marieke Van Erp, Lora Aroyo, Roxane Segers, Lourens Van der Meij, Jacco van Ossenbruggen, Schreiber, Guus, Boband Wielinga, Johan Oomen, and Geertje Jacobs. "Digital Hermeneutics: Agora and the Online Understanding of Cultural Heritage." In Proceedings of the ACM Web Science Conference 11, June 1417 2011, Koblenz, Germany.

Alexander, Arden and Tracy Meehleib. “The Thesaurus for Graphic Materials: Its' History, Use, and Future." Cataloging \& Classification Quarterly 31, no.3-4 (2001): 189-212.

Allen, Rodney and Randall Felton. "Photographs as Historical Documents." OAH Magazine of History 5, no.3 (1991): 7-12.

American Council of Learned Societies. Our Cultural Commonwealth: The Report of the American Council of Learned Societies Commission on Cyberinfrastructure for the Humanities and Social Sciences. New York, 2006.

Anderson, Ian. "Are You Being Served? Historians and the Search for Primary Sources." Archivaria 58 (2004): 88-129.

Armitage, Linda H. and Peter G.B. Enser. "Analysis of User Need in Image Archives." Journal of Information Science 23, no. 4 (1997): 287-299.

Bale, John. “Capturing 'The African' Body? Visual Images and 'Imaginative Sports." Journal of Sport History 25, no. 2 (1998): 235-236.

Banks, Marcus. “Analyzing Images.” In The SAGE Handbook of Qualitative Data Analysis, edited by Uwe Flick, 394-409. London: SAGE Publications, 2014.

Banks, Marcus. "Presenting Research Results." In Visual Methods in Social Research, edited by Marcus Banks, 138-171. London: SAGE Publications, 2001.

Barthes, Roland and Stephen Heath. Image Music Text. New York: Hill and Wang, 1977.

Barthes, Roland. Camera Lucida, New York: Hill and Wang, 1981.

Bateman, Kirklin, Sheila Brennan, Douglas Mudd, and Paula Petric. "Taking a Byte Out of the Archives: Making Technology Work for You." Perspectives: Newsletter of the American Historical Association 43, no. 1 (2005).

Bates, Marcia. "The Cascade of Interactions in the Digital Library Interface." Information Processing and Management 38, no. 3 (2003): 381-400.

Bateson, Gregory. Steps to an Ecology of Mind. Chicago: University of Chicago Press, 1972. 
Batley, Susan. "Visual Information Retrieval: Browsing Strategies in Pictorial Databases." In Online Information 88. Proceedings of the Twelfth International Online Information Meeting, 373-381. Oxford, Learned Information LTD., 1988.

Baxter, Pamela and Susan Jack. "Qualitative Case Study Methodology: Study Design and Implementation for Novice Researchers." The Qualitative Report 13, no. 4 (2008): 554559.

Beard, Charles A. "Written History as an Act of Faith." The American Historical Review 39, no. 2 (1934): 219-231. doi: 10.2307/1838720.

Beattie, Diane. "An Archival User Study: Researchers in the Field of Women's History." Archivaria 29 (1989): 33-50.

Beaudoin, Joan E. “A Framework of Image Use among Archaeologists, Architects, Art Historians and Artists.” Journal of Documentation 70, no.1 (2013): 119-147.

Berkhofer, Robert. Beyond the Great Story: History as Text and Discourse. Cambridge: Harvard University Press, 1997.

Betz-Parker, Elisabeth. Graphic Materials: Rules for Describing Original Items and Historical Collections. Washington, DC: Library of Congress, 1982.

Bignante, Elisa. "The Use of Photo-Elicitation in Field Research.” EchoGeo 11 (2010). doi:10.4000/echogeo.11622.

Blandford, Ann and Simon J. Attfield. Interacting with Information. San Rafael, California: Morgan \& Claypool, 2010.

Block, Herbert. "Well, Everything Helps.” Illustration. 1930 or 1931. From Library of Congress Prints and Photographs Online Catalog. http://www.loc.gov/pictures/item/00652175.

Bloor, Michael and Fiona Wood. "Phenomenological Methods." In Keywords in Qualitative Methods, 128-130. London: Sage Publications, 2006.

Bolick, Cheryl. "Digital Archives: Democratizing the Doing of History." The International Journal of Social Education 21, no. 1 (2006): 122-134.

Boonstra, Onno, Leen Breure and Peter Doorn. "Past, Present, and Future of Historical Information Science." Historical Social Research 29. Amsterdam: Netherlands Institute for Scientific Information, 2004.

Booth, Douglas. "Evidence Revisited: Interpreting Historical Materials in Sport History." Rethinking History 9, no.4 (2005): 475. doi:10.1080/13642520500307990. 
Borgman, Christine. Scholarship in the Digital Age: Information, Infrastructure, and the Internet. Cambridge, Mass: MIT Press, 2007.

Bragg, Molly. “'There is Always More that Can Be Done': A Survey Investigating Libraries' Measurement of Digitized Primary Source Use." MA thesis, University of North Carolina Chapel Hill, 2011. https://cdr.lib.unc.edu/record/uuid:638682ef-5138-49c4-be16c3c8294b08ce.

Braun, Virginia and Victoria Clarke. "Using Thematic Analysis in Psychology." Qualitative Research in Psychology 3, no.2 (2006): 77-101. doi:10.1191/1478088706qp063oa.

Briet, Suzanne. What is Documentation? Edited by Ronald E. Day and Laurent Martinet. Lanham, MD: Scarecrow Press, 2006.

Brockman, William, Laura Neumann, Carole Palmer, and Tonyia Tidline. Scholarly Work in the Humanities and the Evolving Information Environment. Washington, DC: Digital Library Federation and Council on Library and Information Resources, 2002.

Bruce, Christine. The Seven Faces of Information Literacy. Adelaide: Auslib Press, 1997.

Bryman, Alan. Research Methods and Organization Studies. London: Unwin Hyman, 1989.

Buckland, Michael. "Information as Thing." Journal of the American Society for Information Science 42, no. 5 (1991): 351-360.

—. "What is a 'Document'?" Journal of the American Society for Information Science 48, no. 9 (1997): 804-809.

Burger, Barbara Lewis. "Guide to the Holdings of the Still Picture Branch of the National Archives and Records Administration." Washington, DC: National Archives and Records Administration, 1990. http://www.archives.gov/research/guides/still-pictures-guide.html.

Burke, Peter. New Perspectives on Historical Writing. University Park, PA: Pennsylvania State University Press, 1992.

Capurro, Rafael. "Digital Hermeneutics: An Outline.” AI \& Society 25, no.1 (2009): 35-49. doi:10.1007/s00146-009-0255-9.

—. "Foundations of Information Science: Review and Perspectives." 1999. http://www.capurro.de/tampere91.htm

Case, Donald. "The Collection and Use of Information by Some American Historians: A Study of Motives and Methods." Library Quarterly 61, no. 1 (1991): 61-82.

CBPS Sub-Committee on Descriptive Standards. ISAD(G): General International Standard Archival Description - Second Edition. September 2011. http://www.ica.org/en/isadg_general-international-standard-archival-description-second-edition 
Chassanoff, Alexandra. "Historians and the Use of Primary Source Materials in the Digital Age." The American Archivist 76, no.2 (2013): 458-480.

Choemprayong, Songphan and Barbara Wildemuth. "Case Studies.” In Applications of Social Research Methods to Questions in Information and Library Science, 51-61. Edited by Barbara Wildemuth. Westport, CT: Libraries Unlimited, 2009.

Choi, Youngok and Edie M. Rasmussen. "Searching for Images: The Analysis of Users' Queries for Image Retrieval in American History." Journal of the American Society for Information Science and Technology 54, no. 6 (2003): 498-511.

Chung, EunKyung and JungWon Yoon, "Image Needs in the Context of Image Use: An Exploratory Study.” Journal of Information Science 37, no. 2 (2011): 163-177.

Cohen, Julie E. “Cyberspace as/and space.” Columbia Law Journal 107, no. 1 (2007): 210-256.

Cole, Charles. "Information Acquisition in History Ph.D. Students: Inferencing and the Formation of Knowledge Structures.” Library Quarterly 68, no. 1 (1998): 33-54.

Collier Jr., John and Malcolm Collier. Visual Anthropology: Photography as a Research Method. Albuquerque: University of New Mexico Press, 1986.

Collier Jr., John. "Photography in Anthropology: A Report on Two Experiments." American Anthropologist 59 (1957): 843-59.

Collins, Karen. "Providing Subject Access to Images: A Study of User Queries." The American Archivist 61, no. 1 (1998): 36-55.

Conniss, L.R., A.J. Ashford and M.E. Graham. Information Seeking Behavior in Image Retrieval: Visor I Final Report. Institute for Image Data Research, Library and Information Commission Research Report 95. Newcastle upon Tyne, 2000.

Conway, Paul and Ricardo Punzalan. "Fields of Vision: Toward a New Theory of Visual Literacy for Digitized Archival Photographs." Archivaria 71 (2011): 63-97.

Corbin, Juliet and Anselm Strauss. Basics of Qualitative Research: Techniques and Procedures for Developing Grounded Theory. Newbury Park, CA: Sage Publications, 2008.

Cox, Richard J. "Machines in the Archives: Technology and the Coming Transformation of Archival Reference." First Monday, November 2007. http://firstmonday.org/article/view/2029/1894.

Crawford, Walt. MARC for Library Use. Boston, MA: GK Hall, 1989.

Cutter, Charles Ammi. Library Journal 32. New Jersey: R.R. Bowker Company, 1907. 
Dana, John Cotton The Picture Collection. Woodstock, Vermont: Elm Tree Press, 1910.

Danto, Arthur. Narration and Knowledge. New York: Columbia University Press, 2007.

Dooley, Jackie M. and Katherine Luce. Taking Our Pulse: The OCLC Research Survey of Special Collections and Archives. Dublin, Ohio: OCLC Research, 2010.

Dourish, Paul. Where the Action Is: The Foundations of Embodied Interaction. Cambridge, MA: MIT Press, 2001.

Drucker, Johanna. "Intimations of Immateriality: Graphical Form, Textual Sense, and the Electronic Environment." In Reimagining Textuality: Textual Studies in the Late Age of Print, edited by Elizabeth Bergmann Loizeaux and Neil Fraistat, 152-177. Madison: University of Wisconsin, Press, 2002.

—. "Performative Materiality and Theoretical Approaches to Interface." Digital Humanities Quarterly 7, no.1 (2013).

Dublin Core User Guide. http://wiki.dublincore.org/index.php/User_Guide.

Duff, Wendy and Catherine Johnson. "Accidentally Found on Purpose: Information-Seeking Behavior of Historians in Archives." Library Quarterly 72, no. 4 (2002): 472-496.

Duff, Wendy, Barbara Craig, and Joan Cherry. "Finding and Using Archival Resources: A Cross-Canada Survey of Historians Studying Canadian History.” Archivaria 58 (2004): $51-80$.

Duff, Wendy, Emily Monks-Leeson, and Alan Galey. "Contexts Built and Found: a Pilot Study on the Process of Archival Meaning-making." Archival Science 12, no. 1 (2012): 69-92.

Edwards, Elizabeth and Janice Hart, Photographs Objects Histories: On the Materiality of Images. New York: Routledge, 2004.

Edwards, Elizabeth. "Photography and the Material Performance of the Past." History and Theory 48, no.4 (2009): 130-150.

Ernst, Wolfgang. "Distory." In Digital Memory and the Archive, edited by Jussi Parikka, 158171. Minneapolis: University of Minnesota Press, 2013.

Ernst, Wolfgang. "Media Archaeography." In Digital Memory and the Archive, edited by Jussi Parikka, 55-73. Minneapolis: University of Minnesota Press, 2013.

Ernst, Wolfgang. "Media Archaeology as Method of Re/Search in Parallel Lines." Lecture notes, transmediale. 12 "In/compatible" symposium, Haus der Kulturen der Welt, Berlin, Germany, February 5, 2012. https://www.medienwissenschaft.hu- 
berlin.de/de/medienwissenschaft/medientheorien/ernst-inenglish/pdf/transmedial12kurz.pdf

Eskildsen, Kasper. "Leopold Ranke's Archival Turn: Location and Evidence in Modern Historiography." Modern Intellectual History 5, no. 3 (2008): 425-453. doi:10.1017/S1479244308001753.

Farber, Marvin. The Aims of Phenomenology. New York: Harper \& Row, 1966.

Farmer, Sarah. "Going Visual: Holocaust Representation and Method." American Historical Review 115, no. 1 (2010): 115-122.

Fear, Kathleen. "User Understanding of Metadata in Digital Image Collections: Or, What Exactly Do You Mean by 'Coverage'?” The American Archivist 73, no.2 (2010): 26-60.

Fereday, Jennifer and Eimear Muir-Cochrane, "Demonstrating Rigor Using Thematic Analysis: A Hybrid Approach of Inductive and Deductive Coding and Theme Development," International Journal of Qualitative Methods 5, no.1 (2006): 1-11.

Fidel, Raya. "The Image Retrieval Task: Implications for the Design and Evaluation of Image Databases." New Review of Hypermedia and Multimedia 3, no. 1 (1997): 181-199.

Finlay, Linda. “'Outing' the Researcher: The Provenance, Process, and Practice of Reflexivity.” Qualitative Health Research 12, no.4 (2002): 531-545.

Forsyth, William. The Rules of Evidence as Applicable to the Credibility of History. London: Robert Hardwicke, 1874.

Furner, Jonathan. "Information Studies without Information." Library Trends 52, no.3 (2004): $427-446$.

Gabriela Redwine. Personal Digital Archiving. DPC Technology Watch Report 15-01. 2015. doi: http://dx.doi.org/10.7207/twr15-01.

Garcia-Molina, Hector. "Digital Libraries Research Agenda Report: A Strawman Report for the IITA Digital Libraries Workshop." http://dbpubs.stanford.edu:8091/diglib/pub/reports/iita-dlw/appendix2.html.

George, H.B. Historical Evidence. Oxford: Clarendon Press, 1989.

Glaser, Barry and Anselm Strauss, The Discovery of Grounded Theory: Strategies for Qualitative Research. New York: Aldine, 1967.

Gordon, Eric. The Urban Spectator: American Concept Cities from Kodak to Google. Hanover, NH: Dartmouth College Press, 2010. 
Graham, Paul G. "Current Developments in Audiovisual Cataloging." Library Trends 34, no. 1 (1985): 55-66.

Greenberg, Jane. "A Quantitative Categorical Analysis of Metadata Elements in ImageApplicable Metadata Schemas.” Journal of the American Society for Information Science and Technology 52, no. 11 (2001): 917-924.

-. "Intellectual Control of Visual Archives: A Comparison Between the Art and Architecture Thesaurus and the Library of Congress Thesaurus for Graphic Materials." Cataloging \& Classification Quarterly 16, no. 1 (1993): 94.

Guba, Egon. "Criteria for Assessing the Trustworthiness of Naturalistic Inquiries." Educational Communication and Technology 29, no.2 (1981): 75-91.

Hansen, Mark B.N. 'New Media.” In Critical Terms for Media Studies, edited by W.J.T. Mitchell and Mark B.N. Hansen, 172-185. Chicago: The University of Chicago Press, 2010.

Harley, Diane, Jonathan Henke, Shannon Lawrence, Ian Miller, Irene Perciali, and David Nasatir. "Use and Users of Digital Resources: A Focus on Undergraduate Education in the Humanities and Social Sciences." UC Berkeley: Center for Studies in Higher Education (2006).

Harper, Douglas. “Talking about Pictures: A Case for Photo Elicitation.” Visual Studies 17 (2002):13-26.

Harris, Valerie and Peter Hepburn. "Trends in Image Use by Historians and the Implications for Librarians and Archivists." College and Research Libraries 74, no. 3 (2013): 272-287. doi:10.5860/crl-345.

Hartel, Jenna and Leslie Thomson, "Visual Approaches and Photography for the Study of Immediate Information Space," Journal of the American Society for Information Science and Technology 62, no. 11 (2011): 1-11.

Hayles, N. Katherine. Writing Machines. Cambridge: MIT Press, 2002.

Hedstrom, Margaret L., Christopher A Lee, Judith S Olson, and Clifford A Lampe. "'The Old Version Flickers More': Digital Preservation from the User's Perspective." The American Archivist 69 (2006): 159-187.

Heidegger, Martin. Being and Time. Albany: SUNY Press, 1973.

Henige, David. Historical Evidence and Argument. Madison, Wis: University of Wisconsin Press, 2005. 
Hilpert, Zachary. "Ruins Reframed: The Commodification of American Urban Disasters, 18611906." PhD diss., College of William and Mary, 2014.

Hjørland, Birger and Hanne Albrechtson, "Toward a New Horizon in Information Science," Journal of the American Society for Information Science and Technology 46, no.6 (1995): 400-425. doi:0.1002/(SICI)1097-4571(199507)46:6<400::AID-ASI2>3.0.CO;2-Y.

Hjørland, Birger. "Domain Analysis in Information Science: Eleven Approaches - Traditional as Well as Innovative.” Journal of Documentation 58, no. 4 (2002): 422-462.

Hong, Weiyin, James Thong, Wai-man Wong, and Kar-yan Tam. "Determinants of User Acceptance of Digital Libraries: An Empirical Examination of Individual Differences and System Characteristics.” Journal of Management Information Systems 18, vol. 3 (2002): 97-124.

Howell, Martha C. and Walter Prevenier. From Reliable Sources: An Introduction to Historical Methods. Ithaca, N.Y: Cornell University Press, 2001.

Hudgins, Nicole. "A Historical Approach to Family Photography: Class and Individuality in Manchester and Lille, 1850-1914.” Journal of Social History 43, no. 3 (2010): 559-586. doi:10.1353/jsh.0.0298.

Ihde, Don. "More Material Heremenutics." In Yearbook of the Institute for Advanced Study on Science, Technology, and Society. Graz: Profil Verlag, 2004.

Israel, Paul. Edison: A Life of Invention. New York: John Wiley, 1998.

Januarius, Joeri. "Picturing the Everyday Life of Limburg Miners: Photographs as a Historical Source." International Review of Social History 53, no.2 (2008): 293-312. doi:10.1017/S0020859008003453.

Jenkins, Keith. The Postmodern History Reader. London: Routledge, 1997.

Jensen, Mikkel and Mads Søgaard. "Refocusing the Contextual Turn: The Forgotten Construction of Meaning at the Interface." In Proceedings of the Third Nordic Conference on Human-Computer Interaction, 185-188. New York: Association for Computing Machinery, 2004.

Johnson, Allen. The Historian and Historical Evidence. New York: C. Scribner's Sons, 1926.

Jorgensen, Corinne. "Attributes of Images in Describing Tasks." Information Processing \& Management 34, no. 2/3 (1998): 161-174. doi:10.1016/S0306-4573(97)00077-0.

—. "Image Attributes: An Investigation.” PhD diss., Syracuse University, 1995. 
Joseph, Michael. "Information Technology and Access to Visual Images in Printed Books." Visual Resources 11, no.1 (1995): 78-80.

Kaplan, Elisabeth and Jeffrey Mifflin. "'Mind and Sight': Visual Literacy and the Archivist." Archival Issues 21, no. 2 (1996): 73-97.

Kessler, Ben. "Encoding Works and Images: The Story behind VRA Core 4.0." Visual Resources Bulletin 34, no. 1 (2007): 20-33.

Keutel, Marcus and Werner Mellis. “An In-Depth Interpretive Case Study in Requirements Engineering Research: Experiences and Recommendations" MCIS,University of Cologne, 2011.

Krase, Jerome. Seeing Cities Change: Local Culture and Class. Farnham: Ashgate, 2012.

Lacy, Mary A. and Anne Mitchell. "EAD Testing and Implementation at the Library of Congress." The American Archivist 60, no. 4 (1997): 420-435.

Lapenta, Francesco. "The Image as a Form of Sociological Data: A Methodological Approach to the Analysis of Photo-Elicited Interviews." PhD diss., University of London, 2004.

Latour, Bruno. "Beware Your Imagination Leaves Traces." Times Higher Education Literary Supplement, April 6, 2007.

Leary, William H. The Archival Appraisal of Photographs: a RAMP Study with Guidelines. Paris:

Lee, Christopher A. "Digital Curation as Communication Mediation.” In Handbook of Technical Communication, edited by Alexander Mehler, Laurent Romary, and Dafydd Gibbon, 507530. Berlin: Mouton De Gruyter, 2012.

Lewis, George Cornewall. An Inquiry into the Credibility of the Early Roman History. London: J.W. Parker \& Son, 1855.

Library of Congress. Development of the Encoded Archival Description DTD. www.loc.gov/ead/eaddev.html.

-. Introduction to VRA Core 4.0, 2014. http://www.loc.gov/standards/vracore/VRA_Core4_Intro.pdf.

—. MODS User Guidelines Version 3.0. http://www.loc.gov/standards/mods/userguide/

Limberg, Louise. "Phenomenography: A Relational Approach to Research on Information Needs, Seeking, and Use." The New Review of Information Behaviour Research 4, no.1 (2003): 51-67. 
Lincoln, Yvonne and Egon Guba. Naturalistic Inquiry. Beverly Hills, CA: Sage Publications, 1985.

Ma, Jin. "Metadata in ARL Libraries: A Survey of Metadata Practices." Journal of Library Metadata 9, no. 1-2 (2007): 5.

Ma, Lai. "Some Philosophical Considerations in Using Mixed Methods in Library and Information Science Research." Journal of the American Society for Information Science 63, no. 9 (2012):1865.

MacKay, Donald. Information, Mechanism, and Meaning. Cambridge: MIT Press, 1969.

Mai, Jens-Erik. "The Quality and Qualities of Information." Journal of the American Society for Information Science and Technology 64 no.4 (2013): 675-688. doi:10.1002/asi.22783.

Margolis, Eric. "Mining Photographs: Unearthing the Meanings of Historical Photos." Radical History Review 40 (1988): 33-49.

Markey, Karen. "Access to Iconographical Research Collections." Library Trends 37, no. 2 (1988): 154-174.

Markey, Karen. Subject Access to Visual Resources Collections. Greenwood Press: The University of Michigan, 1986.

Markkula, Marjo and Eero Sormumen. "Searching for Photos-Journalists' Practices in Pictorial IR.” Electronic Workshops in Computing. Challenge of Image Retrieval, Newcastle Upon Tyne, February 1998. http://www.bcs.org/upload/pdf/ewic_im98_paper8.pdf.

Maron, Nancy and Sarah Pickle. Appraising our Digital Investment: Sustainability of Digitized Special Collections in ARL Libraries. Washington, DC: Association of Research Libraries, 2013).

Martinez, Katharine. "Imaging the Past: Historians, Visual Images and the Contested Definition of History." Visual Resources 11, no. 1 (1995): 21-45. doi:10.1080/01973762.1995.9658317.

Marton, Ference. "Phenomenography - Describing Conceptions of the World Around Us." Instructional Science 10 (1981): 177-198.

Masur, Louis. "Pictures Have Now Become a Necessity": The Use of Images in American History Textbooks." The Journal of American History 84, no.4 (1998):1409-1424.

Maxwell, Alexander. "Digital Archives and History Research: Feedback from an End-User." Library Review 59, no. 1 (2010):24-39. 
Mccay-Peet, Lori and Elaine Toms. "Image Use Within the Work Task Model: Images as Information and Illustration." Journal of the American Society for Information Science 60, no. 12 (2009): 2416-2429.

McDonough, Jerome. "METS: Standardized Encoding for Digital Library Objects." International Journal on Digital Libraries 6, no.2 (2006): 148-158. http://hdl.handle.net/2142/177.

McGann, Jerome. “Shall These Bones Live?” In The Beauty of Inflections: Literary Investigations in Historical Method and Theory. Oxford: Clarendon Press, 1985.

McHale, John. The Changing Information Environment. Boulder: Westview Press, 1976.

McIntyre, Ronald M and David Woodruff Smith. "Theory of Intentionality." In Husserl's Phenomenology: A Textbook, edited by J.N. Mohanty and William R. McKenna, 147-179. Washington, D.C.: Center for Advanced Research in Phenomenology and University Press of America, 1989.

Miles, Matthew, A. Michael Huberman, and Johnny Saldana. Qualitative Data Analysis: A Methods Sourcebook 3rd edition. Thousand Oaks, California: Sage Publications, 2013.

Miller, Daegan. "Witness Tree: Landscape and Dissent in the Nineteenth Century United States." PhD Diss., Cornell University, 2013.

Miller, Frederic. "Use, Appraisal, and Research: A Case Study of Social History." The American Archivist 49, no. 4 (1981): 371-392.

Miller, Lisa, Stephen K. Galbraith, and RLG Working Group. Capture and Release: Digital Cameras in the Reading Room. Dublin, OH: OCLC Research, 2010.

Mingers, John. "Combining IS Research Methods: Towards a Pluralist Methodology." Information Systems Research 12, no.3 (2001): 240-59.

Muller, Philipp. "Understanding History: Hermeneutics and Source-Criticism in Historical Scholarship." In Reading Primary Sources: The Interpretation of Texts from $19^{\text {th }}$ and 20thCentury History, edited by Miriam Dobson and Benjamin Ziemann, 21-36. Abingdon: Routledge, 2009.

Myers, Michael and David Avison. "Information System Use as a Hermeneutic Process." In Qualitative Research in Information Systems, edited by Michael Myers and David Avison, 224-241. London: Sage Publications, 2002.

Nahl, Diane. "Social-Biological Information Technology: An Integrated Conceptual Framework." Journal of the American Society for Information and Technology 58, no. 13 (2007): 20212046. doi: 10.1002/asi.20690. 
-. "The Centrality of the Affective in Information Behavior." In Information and Emotion: The Emergent Affective Paradigm in Information Behavior Research and Theory, edited by Diane Nahl and Dania Bilal, 3-38. Medford, New Jersey: American Society for Information Science and Technology, 2007.

OCLC/RLG Working Group on Preservation Metadata. "Preservation Metadata for Digital Objects: A Review of the State of the Art." January 31, 2001. https://www.oclc.org/content/dam/research/activities/pmwg/presmeta wp.pdf.

Orbach, Barbara. "So That Others May See So That Others May See: Tools for Cataloging Still Images." Cataloging \& Classification Quarterly 11, no.3-4 (1990): 163-191. doi:10.1300/J104v11n03_08.

Palmer, Carole and Laura Neumann, "The Information Work of Interdisciplinary Humanities Scholars: Exploration and Translation," Library Quarterly 72, no.1 (2002): 85-117.

Palmer, Carole. "Thematic Research Collections." In A Companion to Digital Humanities. Oxford: Blackwell Publishing, 2004.

Panofsky, Erwin. Meaning in the Visual Arts. Garden City, NY: Doubleday Anchor Books, 1955.

Park, Jung-Ran and Yuji Tosaka. "Metadata Creation Practices in Digital Repositories and Collections: Schemata, Selection Criteria, and Interoperability." Information Technology \& Libraries 29, no. 3 (2010): 104-116.

Patton, Michael Quinn. Qualitative Research and Evaluation Methods. $3^{\text {rd }}$ ed. Thousand Oaks, CA: Sage Press, 2002.

PREMIS Editorial Committee. PREMIS Data Dictionary for Preservation Metadata, version 2.0 (March 2008). http://www.loc.gov/standards/premis/v2/premis-dd-2-0.pdf.

Quanchi, Max. "Visual Histories and Photographic Evidence." The Journal of Pacific History 41, no.2 (2006): 165-173. doi:10.1080/00223340600826052.

Ranger, Terence. "Pictures Must Prevail: Sex and the Social History of African Photography in Bulawayo, 1930-1960." Visual History 27 (2001): 261-266.

Ranke, Leopold von. "On the Character of Historical Science." In The Theory and Practice of History, edited by Georg G. Iggers, Konrad von Moltke, Wilhelm Humboldt, and Leopold von Ranke, 14-15. Indianapolis: Bobbs-Merrill, 1973.

Robinson, James H. The New History; Essays Illustrating the Modern Historical Outlook. New York: Macmillan, 1912. 
Rumer, Jennifer and Roger C. Schonfeld. "Supporting the Changing Research Practices of Historians: Final Report from ITHAKA S+R." 2012. doi: http://dx.doi.org/10.18665/sr.22833.

Salo, Edward. "Crossing the Rivers of the State: The Role of the Ferry in the Development of South Carolina, Circa 1680-1920s." PhD diss., Middle Tennessee State University, 2009.

Sampsell-Willman, Kate. "Using Lewis Hine's Child Labor Photographs, Part 2: The Miners." The Journal of the Gilded Age and Progressive Era Online.January 25, 2011. http://www.shgape.org/supplement-using-lewis-hine-child-labor-photographs-part-two$\underline{\text { miners. }}$

—. Lewis Hine as Social Critic. Jackson: University Press of Mississippi, 2009.

Schlack, Tim. "Framing Photographs, Denying Archives: The Difficulty of Focusing on Archival Photographs." Archival Science 8, no. 2 (2008): 85-101.

Scholz, Roland and Olaf Tietjie. "Types of Case Studies." In Embedded Case Study Methods: Integrating Quantitative and Qualitative Knowledge, edited by Roland Scholz and Olaf Tietjie, 9-14. Thousand Oaks, CA: Sage Publications, 2002.

Schwartz, Joan M. "Coming to Terms with Photographs: Descriptive Standards, Linguistic 'Othering,' and the Margins of Archivy." Archivaria 54 (2002): 142-171.

Segal, Jane and Lisa Spiro. "The Impact of Digital Resources on Humanities Scholarship.” Rice University, 2007. http://library.rice.edu/services/dmc/about/projects/the-impact-ofdigital-resources-on-humanities-research.

Shannon, Claude E. "The Mathematical Theory of Communication." The Bell System Technical Journal 27 (1948): 379-423.

Shatford-Layne, Sara. "Analyzing the Subject of a Picture: A Theoretical Approach." Cataloging \& Classification Quarterly 6, no. 3 (March 1986): 39-62. doi:10.1300/J104v06n03_04.

—. "Some Issues in the Indexing of Images." Journal of the American Society for Information Science 45, no. 8 (1994): 583-588.

Shaw, Renata V. A Century of Photographs, 1846-1946. Washington DC: The Library of Congress, 1980.

Sheble, Laura and Barbara Wildemuth. "Transaction Logs." In Applications of Social Research Methods to Questions in Information and Library Science, edited by Barbara Wildemuth, 166-177. Westport, CT: Libraries Unlimited, 2009.

Shenton, Andrew K. "Strategies for Ensuring Trustworthiness in Qualitative Research Projects." Education for Information 22 (2004): 63-75. 
Simcox, William Henry. Principles of Historical Evidence Considered in Their Bearing on the History of Remote Times. Oxford: T. \& G. Shrimpton, 1868.

Sinn, Donghee. "Impact of Digital Archival Collections on Historical Research." Journal of the American Society for Information Science and Technology 63, no. 8 (2012): 1521-1537.

Smith, Barry. Cambridge Companion to Husserl. Cambridge: Cambridge University Press, 1996.

Smith, Marian. "Young People: A Phenomenographic Investigation Into the Ways They Experience Information.” PhD diss., Loughborough University, 2010.

Snyder, Jamie. "Image-Enabled Discourse: Investigating the Creation of Visual Information as Communicative Practice.” PhD diss., Syracuse University, 2012.

Solomon, Paul. "Discovering Information in Context." Annual Review of Information Science and Technology 36, no.1 (2005): 229-264.

Soloski, John. "Meaning and Information: A Perspective on Donald M. MacKay." Journal of Communication Inquiry 2, no. 2 (1977): 51-59.

Sontag, Susan. On Photography. New York: Farrar, Straus and Giroux, 1977.

Spink, Amanda and Charles Cole. "Human Information Behavior: Integrating Diverse Approaches and Information Use." Journal of the American Society for Information Science and Technology 57, no. 1 (2006): 25-35. doi: 10.1002/asi.20249.

Stake, Robert E. The Art of Case Study Research. Thousand Oaks, California: Sage Publications, 1995.

-. "Qualitative Case Studies." In The SAGE Handbook of Qualitative Research, edited by Norman Denzin and Yvonna Lincoln, 435-454. Thousand Oaks, CA: Sage Publications, 2000 .

Stearns, Peter and Jan Lewis. An Emotional History of the U.S. New York: NYU Press, 1998.

Sternfeld, Joshua. "Archival Theory and Digital Historiography: Selection, Search, and Metadata as Archival Processes for Assessing Historical Contextualization." The American Archivist 74 (2011): 544-575.

Stieg-Dalton, Margaret and Laurie Charnigo. "Historians and Their Information Sources." College and Research Libraries 65, no. 4 (2004): 400-425.

Stoan, Stephen. "Research and Information Retrieval among Academic Researchers." Library Trends 39, no. 3 (Winter 1991): 238-258. http://hdl.handle.net/2142/7725. 
Stone, Lawrence. The Past and the Present Revisited. London: Routledge \& Kegan Paul, 1987.

Stone, Sue. "Humanities Scholars: Information Needs and Uses." Journal of Documentation 38, no. 4 (1982): 292-313. http://www.emeraldinsight.com/doi/abs/10.1108/eb026734.

Suter, W. Newton. Introduction to Educational Research: A Critical Thinking Approach. Thousand Oaks, California: SAGE Publications, 2006.

Taylor, Bradley. "The Effect of Surrogation on Viewer Response to Expressional Qualities in Works of Art." PhD Diss., University of Michigan, 2001.

Taylor, Robert. "Information Use Environments." In Progress in Communication Sciences Vol. 10, edited by Brenda Dervin and M.J. Voight, 217-225. Norwood, NJ: Ablex Publishing Corp, 1991.

-. Value-added Processes in Information Systems. New Jersey: Ablex Publishing Corporation, 1986.

Terras, Melissa. "Digital Curiosities: Resource Creation via Amateur Digitization." Literary and Linguistic Computing 25, no.4 (2010): 425-438. doi:10.1093/llc/fqq019.

Theiler, Janine M. "A Shared Story of Successful Spanish Learning: An Embedded Multiple Case Study". PhD diss, 2012.

Thomas, James and Angela Harden, "Methods for the Thematic Synthesis of Qualitative Research in Systematic Reviews." BMC Medical Research Methodology 8, no.45 (2008).

Thomas, Joe D. "Photographic Archives.” The American Archivist 21, no.4 (1958): 419-424.

Thomas, Julia Adeney. “The Evidence of Sight.” History and Theory 48 (2009): 151-168.

Thompson, Ruth. "The Collection and Preservation of Local Historical Pictures in the Minneapolis Public Library." The American Archivist 9, no. 4 (1946): 219-225.

Tibbo, Helen. "Primarily History in America: How US Historians Search for Primary Materials at the Dawn of the Digital Age." The American Archivist 66, no. 1 (2003): 9-50

Troncale, Anthony T. "Worth Beyond Words: Romana Javitz and the New York Public Library's Picture Collection.” New York Public Library Website. Retrieved from http://www.nypl.org/locations/tid/45/node/62019.

Turner, Kristen. “Opera in English: Class and culture, 1878-1910.” PhD diss., University of North Carolina, 2015.

United States, David Fitzgerald, and A. W. Greely. List of the Photographs and Photographic Negatives Relating to the War for the Union, Now in the War Department Library. 
Washington: Government Printing Office, 1897.

https://archive.org/details/listofphotograph00unit

van Hage, Willem, Veronique Malaise, Roxane Segers, Laura Hollink, and Guus Schreiber. "Design and Use of the Simple Event Model (SEM)." Web Semantics: Science, Services and Agents on the World Wide Web 9 (2011): 128-136.

Visual Resources Association. Cataloging Cultural Objects. http://cco.vrafoundation.org/.

-. Core Categories for Visual Resources Version 4.0. 2007. http://www.vraweb.org/project/vracore4/index.html.

W3C. Resource Description Framework. https://www.w3.org/RDF/.

Walker, Jonathan. "Let Us Burn the Gondolas: Venice as a Modern City." Rethinking History 15, no.1 (2011): 3-32. doi:10.1080/13642529.2011.546128.

Walsham, Geoff. "Interpretive Case Studies in IS Research: Nature and Method." European Journal of Information Systems 4 (1995): 74-81. doi: 10.1057/ejis.1995.9.

Warwick, Claire, Melissa Terras, Paul Huntington, and Nikoleta Pappa, "If You Build It Will They Come? The LAIRAH Study: Quantifying the Use of Online Resources in the Arts and Humanities through Statistical Analysis of User Log Data." Literary and Linguistic Computing 23, no.1 (2007): 85-102. doi: 10.1093/llc/fqm045.

Weibel, Stuart and Eric Miller. "Image Description on the Internet: A Summary of the CNI/OCLC Image Metadata Workshop." D-Lib Magazine, January 1997. http://www.dlib.org/dlib/january97/oclc/01 weibel.html.

Weintraub, Karl. "The Humanistic Scholar and the Library." The Library Quarterly 50, no. 1 (1980): 22-39.

White, Hayden. "Historiography and Historiphoty." The American Historical Review 93, no. 5 (1988): 1194.

Willis, Gordon. Cognitive Interviewing: A Tool for Improving Survey Design. Thousand Oaks, CA: Sage Publications, 2005.

—. "Cognitive Interviewing: A 'How To' Guide." 1999 Meeting of the American Statistical Association. Research Triangle Park, NC: Research Triangle Institute, 1999, 7.

Wilson, T.D. "Alfred Schutz, Phenomenology and Research Methodology for Information Behaviour Research." New Review of Information Behaviour Research 3 (2002): 71-82. 
Wineburg, Samuel. "Historical Problem Solving: A Study of the Cognitive Processes Used in the Evaluation of Documentary and Pictorial Evidence." Journal of Educational Psychology 83, no.1 (1991): 73-87.

-. "Historical Problem-Solving: A Study of the Cognitive Processes Used in the Evaluation of Documentary Evidence.” PhD diss., Stanford University, 1990.

Yakel, Elizabeth and Deborah Torres. "AI: Archival Intelligence and User Expertise." The American Archivist 66, no. 1 (2003): 51-78.

Yin, Robert. Case Study Research: Design and Methods $2^{\text {nd }}$ edition. Thousand Oaks, CA: Sage Publishing, 1994.

Zhang, Ying and Yuelin Li. "A User-Centered Functional Metadata Evaluation of Moving Image Collections." Journal of the American Society for Information Science and Technology 59, no. 8 (2008): 1331-1346. doi:10.1002/asi.20839.

Zinkham, Helena, Patricia D. Cloud, and Hope Mayo. "Providing Access by Form of Material, Genre, and Physical Characteristics: Benefits and Techniques." The American Archivist 52, no.3 (1989): 300-319. http://dx.doi.org/10.17723/aarc.52.3.g657371200612642.

Zinkham, Helena. "Reading and Researching Photographs." In Photographs: Archival Care and Management, edited by Mary Lynn Ritzenthaler and Diane Vogt-O'Connor, 59-77. Chicago: The Society of American Archivists, 2006.

Zunde, Pranas. "Selected Bibliography o+A20n Information Theory Applications to Information Science and Related Subject Areas." Information Processing and Management 20, no.3 (1984): 417-497. 